$\mathrm{DOE} / \mathrm{ET} / 51013--292$

DE91 016969

PFC/RR-91-9

A COMPARISON Of RADIOACTIVE WASTE from FIRST GENERATION FUSION REACTORS and FAST FISSION REACTORS with ACTINIDE RECYCLING*

M. Koch and M.S. Kazimi

April 1991

\begin{abstract}
Plasma Fusion Center and

Department of Nuclear Engineering

Massachusetts Institute of Technology

Cambridge, MA 02139 U.S.A.
\end{abstract}

*This work was partially supported by EG\&G Idaho, Inc. and the U.S. Department of Energy under DOE Contract

No. DE-AC02-78ET-51013 and DE-FG02-91ER-54110

Reproduction, translation, publication, use and disposal in whole or in part, by or for the United States government is permitted 


\section{DISCLAIMER}

This report was prepared as an account of work sponsored by an agency of the United States Government. Neither the United States Government nor any agency Thereof, nor any of their employees, makes any warranty, express or implied, or assumes any legal liability or responsibility for the accuracy, completeness, or usefulness of any information, apparatus, product, or process disclosed, or represents that its use would not infringe privately owned rights. Reference herein to any specific commercial product, process, or service by trade name, trademark, manufacturer, or otherwise does not necessarily constitute or imply its endorsement, recommendation, or favoring by the United States Government or any agency thereof. The views and opinions of authors expressed herein do not necessarily state or reflect those of the United States Government or any agency thereof. 


\section{DISCLAIMER}

Portions of this document may be illegible in electronic image products. Images are produced from the best available original document. 


\title{
A Comparison of Radioactive Waste from First Generation Fusion Reactors and Fast Fission Reactors with Actinide Recycling
}

\author{
by \\ M. Koch and M.S Kazimi
}

\begin{abstract}
Limitations of the fission fuel resources will presumably mandate the replacement of thermal fission reactors by fast fission reactors that operate on a selfsufficient closed fuel cycle. This replacement might take place within the next one hundred years, so the direct competitors of fusion reactors will be fission reactors of the latter rather than the former type. Also, fast fission reactors, in contrast to thermal fission reactors, have the potential for transmuting longlived actinides into short-lived fission products. The associated reduction of the long-term activation of radioactive waste due to actinides makes the comparison of radioactive waste from fast fission reactors to that from fusion reactors more rewarding than the comparison of radioactive waste from thermal fission reactors to that from fusion reactors.
\end{abstract}

Radioactive waste from an experimental and a commercial fast fission reactor and an experimental and a commercial fusion reactor has been characterized. The fast fission reactors chosen for this study were the Experimental Breeder Reactor $I I$ (EBR-II) and the Integral Fast Reactor (IFR). The fusion reactors chosen for this study were the International Thermonuclear Experimental Reactor (ITER) and a Reduced Activation Ferrite Helium Tokamak (RAFHT).

The four reactors considered operate on an idealized self-sufficient closed fuel cycle, i.e. actinides and tritium are regarded as fuel and recycled back to the reactor. In the case of the two fast fission reactors, actinide recycling is possible without detrimental effects to the neutronics, because at the very high average neutron energies in these reactors, not only plutonium, but also most other actinides become fissionable, i.e. constitute fuel rather than poison. Realistically, the radioactive waste from the two fast fission reactors will contain some actinides and that from the two fusion reactors will contain some tritium. However, since actual separation efficiencies are expected to be in the $99.9 \%$ range, the radioactive waste will contain less than $0.1 \%$ of the actinides or the tritium. In contrast, thermal fission reactors do not operate on a self-sufficient closed fuel cycle and hence their radioactive waste contains up to $100 \%$ of the actinides.

The fast fission and the fusion reactors have been approximated as a set of homogenized reactor components of simple cylindrical and/or hexagonal geometry. Reactor components as radioactive waste were characterized by several 
parameters. These parameters describe the volume and activation of radioactive waste and are pertinent to US regulatory standards.

Build-up and decay of radionuclides in reactor components were simulated by the computer codes ORIGEN-II for fast fission reactors and ONEDANT and $R E A C$-II for fusion reactors. Auxiliary computer codes were developed to convert the output of those three computer codes into radioactive waste parameters. The parameters were not normalized to the different power levels of the compared reactors, but rather evaluated for these reactors as built.

The comparison of radioactive waste parameters shows that radioactive waste from the experimental fast fission reactor may be less hazardous than that from the experimental fusion reactor. Inclusion of the actinides would reverse this conclusion only in the long-term. Radioactive waste from the commercial fusion reactor may always be less hazardous than that from the commercial fast fission reactor, irrespective of the inclusion or exclusion of the actinides. The fusion waste would even be far less hazardous, if advanced structural materials, like silicon carbide or vanadium alloy, were employed.

Also, radioactive waste from the experimental fast fission reactor may be less hazardous than that from the commercial fast fission reactor. This is a direct consequence of the utilization of highly ${ }^{235} \mathrm{U}$ enriched fuel in $E B R-I I$ resulting in a lower activation than the utilization of uranium-plutonium-minor-actinides fuel in IFR. Radioactive waste from the commercial fusion reactor may be less hazardous than that from the experimental fusion reactor. This is a direct consequence of the utilization of standard materials (SS316) in ITER resulting in a higher activation than the utilization of Reduced Activation Materials (RAF) in $R A F H T$. The generation of High Level Radioactive Waste (HLRW) is likely not to be avoided even for $R A F H T$.

The volume of radioactive waste from the two fusion reactors is larger than the volume of radioactive waste from the two fast fission reactors. Material selection in the fusion reactors plays a far more important role in controlling the activation of the radioactive waste than it does in the fast fission reactors. If recycling of fusion reactor structural materials is found feasible in the future, the volume of radioactive waste from fusion reactors will be reduced. 


\section{Acknowledgements}

This report is based on the thesis submitted by the first author to the MIT Department of Nuclear Engineering in partial fulfillment of the requirements for the degree of Master of Science in Nuclear Engineering.

Our gratitude is directed to Robert Hill from the Argonne National Laboratory and Yousry Gohar from the Argonne National Laboratory, who kept us up to date on the Integral Fast Reactor and the International Thermonuclear Experimental Reactor, respectively.

Bhavya Lal from the Massachusetts Institute of Technology and Fred Mann from the Hanford Engineering Development Laboratory were of valuable help in the proper application of the ORIGEN-II and REAC-II codes.

Our special gratitude goes to John Massidda from Grumman Space Systems, who provided literally invaluable help in all aspects of the application of computer codes. The same holds true for Robert Thayer and Mark London from the MIT Plasma Fusion Center.

The comments provided by Stephen Herring and Steven Piet of EGEG Idaho, Inc. on a draft of this report are greatly appreciated.

Elizabeth Parmelee from the MIT Department of Nuclear Engineering and Janet Anderson from the MIT Plasma Fusion Center contributed great administrative support.

We gratefully acknowledge partial funding for this report provided by the Office of Magnetic Fusion Energy of the U.S. Department of Energy. 


\section{Contents}

1 Introduction 15

1.1 Topic \& Motivation . . . . . . . . . . . . . . 15

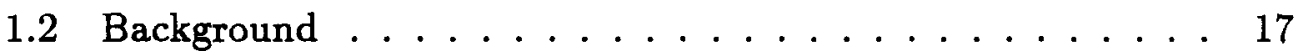

1.3 Focus . . . . . . . . . . . . . . . . 19

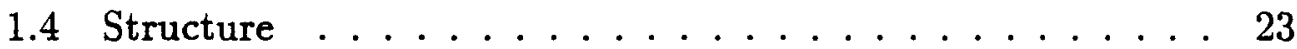

1.5 Previous Studies . . . . . . . . . . . . . 24

2 Radioactive Waste Parameters 26

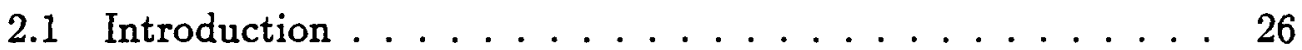

2.2 Volume. . . . . . . . . . . . . . . . . 30

2.3 Radioactivity . . . . . . . . . . . . . 31

2.4 Whole Body $\gamma$-Dose Rate . . . . . . . . . . . . 32

2.5 Decay power . . . . . . . . . . . . . . . . 35

2.6 Biological Hazard Potential $\ldots \ldots \ldots \ldots$

2.7 Radioactive Waste Classification . . . . . . . . . . . . . 43

2.7.1 Low, Intermediate and High Level Radioactive Waste 43

2.7.2 Class A, Class B, Class C and Class D Radioactive Waste . . . . . . . . . . . . . 45

2.8 Intruder Dose Rate . . . . . . . . . . . . . . . . . 48

2.8 .1 Construction Scenario . . . . . . . . . . . 52

2.8 .2 Agriculture Scenario . . . . . . . . . . . 5 56

3 Methods of the Study $\quad 60$

3.1 Introduction . . . . . . . . . . . . . . 60

3.2 Fast Fission Reactors . . . . . . . . . . . . . . . . 61

3.2.1 ORIGEN-II Neutron Activation Code . . . . . . . . 61 
3.3 Fusion Reactors . . . . . . . . . . . . . . . . . 62

3.3 .1 VMIBOB Code . . . . . . . . . . . . . . 62

3.3.2 ONEDANT Neutron Transportation Code ..... . 65

3.3 .3 FLXWRT Code . . . . . . . . . . . . . . . 67

3.3.4 AVEFLUX Code . . . . . . . . . . . . . 67

3.3.5 COMCOMP Code . . . . . . . . . . . . . 69

3.3.6 REAC-II Neutron Activation Code . . . . . . . . 75

3.4 OPCPOST \& RECPOST Codes . . . . . . . . . . . . . 76

3.5 Integration of the Codes $\ldots \ldots \ldots \ldots \ldots$

3.5.1 Fast Fission Reactors . . . . . . . . . . . . . 78

3.5 .2 Fusion Reactors . . . . . . . . . . . . . . 79

4 Description of the Reactors $\quad 81$

4.1 Introduction . . . . . . . . . . . . 81

4.2 Fast Fission Reactors . . . . . . . . . . . . . . . . 82

4.2.1 The Experimental Breeder Reactor II . . . . . . . . 83

4.2.1.1 History \& Objectives . . . . . . . . . . . 83

4.2.1.2 Operation Schedule . . . . . . . . . . 86

4.2.1.3 Design . . . . . . . . . . . . . 88

4.2.2 The Integral Fast Reactor . . . . . . . . . . . . . 92

4.2.2.1 History \& Objectives . . . . . . . . . . . . 92

4.2.2.2 Operation Schedule . . . . . . . . . . . 93

4.2.2.3 Design . . . . . . . . . . . . . 93

4.3 Fusion Reactors . . . . . . . . . . . . . . . . . . . . 94

4.3.1 The International Thermonuclear Experimental Reactor . . . . . . . . . . . . . . 9 97

4.3.1.1 History \& Objectives . . . . . . . . . . 97

4.3.1.2 Operation Schedule . . . . . . . . . . 9 98

4.3.1.3 Design . . . . . . . . . . . . . . 100

4.3.2 The Reduced Activation Ferrite Helium Tokamak . . 104 4.3.2.1 History \& Objectives . . . . . . . . . . . 104

4.3.2.2 Operation Schedule . . . . . . . . . . . 104

4.3.2.3 Design . . . . . . . . . . . . . 105

4.4 Radioactive Waste Processing for Fast Fission Reactors . . . 105 
4.4.1 EBR-II and IFR Pyroprocesses . . . . . . . . 105

4.4 .2 Basic Chemistry . . . . . . . . . . . 108

4.4 .3 EBR-II Pyroprocess . . . . . . . . . . . 110

4.4.3.1 Melt-refining Process . . . . . . . . . . 110

4.4.3.2 Recovering Process . . . . . . . . . . . . 112

4.4.3.2.1 Skull Oxidation Step . . . . . . . 112

4.4.3.2.2 Skull Reclamation Step . . . . . 113

4.4 .4 IFR Pyroprocess . . . . . . . . . . . . . 113

4.4.4.1 Electro-refining Process . . . . . . . . 113

4.4.4.1.1 Dissolution Step . . . . . . 113

4.4.4.1.2 Separation Step . . . . . . . . 115

4.4.4.2 Purifying Process . . . . . . . . . . . 116

4.4.4.3 Extracting and Stripping Process . . . . . 117

5 Comparison of the Reactors 120

5.1 Introduction . . . . . . . . . . . . . 120

5.1.1 Reactor Components as Radioactive Waste . . . . . 120

5.1.2 Cycle, Life-time \& Decommissioning Radioactive Waste 127

5.1.3 Presentation of the Comparison of Radioactive Waste 132

5.2 Volume . . . . . . . . . . . . . . . . . 133

5.3 Radioactivity . . . . . . . . . . . . . 135

5.4 Whole Body $\gamma$-Dose Rate . . . . . . . . . . . . . 144

5.5 Decay Power . . . . . . . . . . . . . . . . . . 145

5.6 Biological Hazard Potential . . . . . . . . . . . 153

5.7 Radioactive Waste Classification . . . . . . . . . . . 153

5.8 Intruder Dose Rate . . . . . . . . . . . . . . . . . 162

5.8.1 Construction Scenario . . . . . . . . . . . 162

5.8 .2 Agriculture Scenario . . . . . . . . . . . . 165

5.9 Radionuclides in Radioactive Waste . . . . . . . . . 168

6 Summary and Conclusion $\quad 170$

6.1 Summary ................... 170 
6.2 Conclusion.................... 172

A Reactor Materials and Geometries $\quad 185$

$\begin{array}{ll}\text { B Radioactive Waste Parameters } & 201\end{array}$ 


\section{List of Figures}

5.1 Comparison of Specific Radioactivity of Fission Products, Activation Products and Actinides, EBRII and IFR . . . . 124

5.2 Comparison of Specific Decay Power of Fission Products, Activation Products and Actinides, EBRII and IFR . . . . . 125

5.3 Comparison of Specific Biological Hazard Potential of Fission Products, Activation Products and Actinides, EBRII and IFR 126

5.4 Specific Radioactivity EBR-II vs. ITER . . . . . . . 138

5.5 Specific Radioactivity IFR vs. RAFHT . . . . . . . . . 139

5.6 Life Time Absolute Radioactivity EBR-II vs. ITER . . . . . 141

5.7 Life Time Absolute Radioactivity IFR vs. RAFHT . . . . 142

5.8 Life Time Absolute $\gamma$-Dose Rate EBR-II vs. ITER . . . 146

5.9 Life Time Absolute $\gamma$-Dose Rate IFR vs. RAFHT . . . . . 147

5.10 Specific Decay Power EBR-II vs. ITER . . . . . . . . . 148

5.11 Specific Decay Power IFR vs. RAFHT . . . . . . . . . . 149

5.12 Life Time Absolute Decay Power EBR-II vs. ITER . . . . . 151

5.13 Life Time Absolute Decay Power IFR vs. RAFHT . . . . . 152

5.14 Specific Biological Hazard Potential EBR-II vs. ITER . . . 154

5.15 Specific Biological Hazard Potential IFR vs. RAFHT . . . 155

5.16 Life Time Absolute Biological Hazard Potential EBR-II vs. ITER . . . . . . . . . . . . . . . . 156

5.17 Life Time Absolute Biological Hazard Potential IFR vs. RAFHT157

5.18 Radioactive Waste Class $C$ Index, EBR-II vs. ITER . . . . 159

5.19 Radioactive Waste Class $C$ Index, IFR vs. RAFHT . . . 160 
5.20 Intruder Dose Rate, Construction Scenario, EBR-II vs. ITER 163

5.21 Intruder Dose Rate, Construction Scenario, IFR vs. RAFHT 164

5.22 Intruder Dose Rate, Agriculture Scenario, EBR-II vs. ITER 166

5.23 Intruder Dose Rate, Agriculture Scenario, IFR vs. RAFHT . 167

B.1 Reactor Specific Radioactivity for EBR-II, IFR, ITER and RAFHT . . . . . . . . . . . . . . 212

B.2 Reactor Life Time Absolute Radioactivity for $E B R-I I, I F R$, ITER and RAFHT . . . . . . . . . . . 213

B.3 Reactor Specific Decay Power for EBR-II, IFR, ITER and RAFHT . . . . . . . . . . . . . . . 214

B.4 Reactor Life Time Absolute Decay Power for EBR-II, IFR,

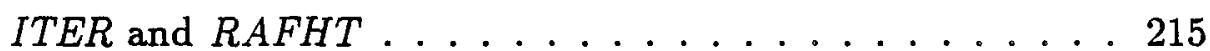




\section{List of Tables}

2.1 Radiation Environments according to 10CFR20, Adapted from [47] ................... 37

2.2 Meaning and Dimension of $P D C$, Barrier and Auxiliary Barrier Factors, Adapted from [8] . . . . . . . . . . . 51

2.3 Barrier and Auxiliary Barrier Factors, Construction Scenario 53

2.4 Auxiliary Barrier Factors used in this Study, Construction Scenario . . . . . . . . . . . . . . 55

2.5 Barrier and Auxiliary Barrier Factors, Agriculture Scenario $\quad 57$

2.6 Auxiliary Barrier Factors used in this Study, Agriculture Scenario . . . . . . . . . . . . . 59

4.1 Volumes and Masses of Experimental Breeder Reactor II Clad, Duct and Fuel Components, Adapted from [44,53,54]. 91

4.2 Volumes and Masses of Experimental Breeder Reactor II Shield Component, Adapted from $[44,53,54] \ldots . . . . .92$

4.3 Volumes and Masses of Integral Fast Reactor Clad, Duct and Fuel Components, Adapted from $[14,17,25,34,35,51] \ldots . .95$

4.4 Volumes and Masses of Integral Fast Reactor Reflector and Shield Components, Adapted from $[14,17,25,34,35,51] \ldots$. . 96

4.5 Stages of ITER Physics Phase, Adapted from [21,22] . . . 98

4.6 Substages of Stage 3 of ITER Physics Phase, Adapted from $[21,22] \ldots \ldots \ldots \ldots \ldots \ldots$

4.7 Average Neutron First Wall Load of ITER Operation Phases 100 
4.8 Volumes and Masses of International Thermonuclear Experimental Reactor Components, Adapted from [11,21] . . . . . 102

4.9 Weight Densities and Solid Volume Fractions of International Thermonuclear Experimental Reactor Components, Adapted from $[11,21] \ldots \ldots \ldots . . \ldots . . . \ldots 103$

4.10 Volumes and Masses, Weight Densities and Solid Volume Fractions of Reduced Activation Ferrite Helium Tokamak Components, Adapted from [19] . . . . . . . . . 106

4.11 Oxidation and Reduction Potentials, Adapted from [3,5] . . 108

4.12 Free Energy of Formation at $500^{\circ} \mathrm{C}$, Adapted from [5] . . . 111

5.1 Summary of Operation Schedules . . . . . . . . . . . 129

5.2 Definition of Cycle, Life-time and Decommissioning Radioactive Waste . . . . . . . . . . . . . . . . . . 131

5.3 Volumes of Cycle, Life Time and Decommissioning Radioactive Waste . . . . . . . . . . . . . . . . . . . . . 134

A.1 Element Composition of Assembly of Experimental Breeder Reactor II Clad Component . . . . . . . . . . . . . . 186

A.2 Element Composition of Assembly of Experimental Breeder Reactor II Duct Component . . . . . . . . . . . . . . 186

A.3 Isotope and Element Composition of Assembly of Experimental Breeder Reactor II Fuel Component, Adapted from [44] . . . . . . . . . . . . . . . 18

A.4 Element Composition of $100 \%$ of Experimental Breeder Reactor II Shield Component . . . . . . . . . . . . . . . . 188

A.5 Element Composition of $100 \%$ of Integral Fast Reactor Clad Component . . . . . . . . . . . . . . . . 188

A.6 Element Composition of $100 \%$ of Integral Fast Reactor Duct Component . . . . . . . . . . . . . . . 189 
A.7 Isotope and Element Composition of $25 \%$ of Integral Fast Reactor Fuel Component, Adapted from [51] . . . . . . . 190

A.8 Element Composition of $100 \%$ of Integral Fast Reactor Reflector and Shield Components . . . . . . . . . . . . 191

A.9 Material Composition of International Thermonuclear Experimental Reactor Components, Adapted from [11,21,22] . 192

A.10 Radial Build of International Thermonuclear Experimental Reactor Inboard Components, Adapted from [11,21] . . . . 193

A.11 Radial Build of International Thermonuclear Experimental Reactor Inboard Components, Continued, Adapted from [11,21]194

A.12 Radial Build of International Thermonuclear Experimental Reactor Outboard Components, Adapted from $[11,21]$. . . . 195

A.13 Radial Build of International Thermonuclear Experimental Reactor Outboard Components, Continued, Adapted from $[11,21] \ldots \ldots \ldots \ldots \ldots$. . . . . . . . 196

A.14 Material Composition of Reduced Activation Ferrite Helium Tokamak Components, Adapted from [19] . . . . . . . 197

A.15 Radial Build of Reduced Activation Ferrite Helium Tokamak Inboard Components, Adapted from [19] . . . . . . . . . 197

A.16 Radial Build of Reduced Activation Ferrite Helium Tokamak Outboard Components, Adapted from [19] . . . . . . . 198

A.17 Element Composition of Structure Material, Adapted from $[12,17,19] \ldots \ldots \ldots$

A.18 Element Composition of Conductor and Breeder Material, Adapted from [19] . . . . . . . . . . . . . . . . 200

B.1 Specific Radioactive Waste Parameters at 10years after Discharge . . . . . . . . . . . . . . . . . . . 202

B.2 Specific Radioactive Waste Parameters at 100years after Discharge . . . . . . . . . . . . . . . . . . 203 
B.3 Specific Radioactive Waste Parameters at 1000years after Discharge . . . . . . . . . . . . . . . . . . . 204

B.4 Absolute Radioactive Waste Parameters for Cycle Radioactive Waste at 10years after Discharge . . . . . . . 205

B.5 Absolute Radioactive Waste Parameters for Life Time Radioactive Waste at 10years after Discharge . . . . . . 206

B.6 Absolute Radioactive Waste Parameters for Decommission Radioactive Waste at 10years after Discharge . . . . . . . 207

B.7 Absolute Radioactive Waste Parameters for Cycle Radioactive Waste at 100 years after Discharge . . . . . . . . . 208

B.8 Absolute Radioactive Waste Parameters for Life Time Radioactive Waste at 100 years after Discharge . . . . . . . 209

B.9 Absolute Radioactive Waste Parameters for Cycle Radioactive Waste at 1000years after Discharge . . . . . . . 210

B.10 Absolute Radioactive Waste Parameters for Life Time Radioactive Waste at 1000years after Discharge . . . . . . 211 


\section{Chapter 1}

\section{Introduction}

\subsection{Topic \& Motivation}

The energy supply structure of a society must be based on diverse energy conversion systems in order to guarantee the efficiency, flexibility and reliability of this structure. A typical such structure may consist of coal, oil, gas, nuclear and solar energy conversion systems. These different systems have different impacts on the environment.

One of the most important problems with respect to the environmental impact of nuclear energy conversion systems consists of the radioactive waste that is inevitably generated by them. The principal techniques to handle radioactive waste and store it for the short-term and the intermediateterm are known. Such waste management is accomplished at many facilities in the United States (Hanford, Savannah River), Great Britain (Sellafield), the Federal Republic of Germany (Karlsruhe, Asse, Gorleben), Sweden (Forsmark) and France (LaHague) [4].

However, considerable uncertainties exist with respect to the best approach to be used for long-term storage of radioactive waste. Those uncertainties are reflected in the economical and political indecision on nuclear energy in general and hence create a virtual deadlock in the process of finding a solution to this problem. Thus it is important in this context to 
minimize the need for long-term radioactive waste storage.

It must be noted that the radioactive waste produced by nuclear fission and nuclear fusion - the two forms of nuclear energy - is generally different, especially in the long-term. Furthermore, it is well recognized that radioactive waste from thermal and fast fission reactors with once-through fuel cycle is more hazardous than that from fusion reactors, basically because of the high content of long lived-actinides.

However, advanced fast fission reactors offer the opportunity of separating the actinides from the radioactive waste and recycling them back to the reactor $[5,44]$. The comparison conducted in this study determines the features of radioactive waste from such advanced fast fission reactors and from fusion reactors. The study might thus provide evidence that radioactive waste from advanced fast fission reactors is not inherently more hazardous than that from fusion reactors.

Unlike actinide separation and recycling, separating long-lived nuclides from the radioactive waste of fusion reactors and recycling them back to the reactor has not been demonstrated yet and therefore will not be considered for this study. Nevertheless, theoretical concepts for such kind of separation and recycling do exist [50].

This comparative study of waste is important in view of the upcoming need for clean but efficient energy conversion systems to satisfy the world's ever growing demand for energy. However, following the diversity principle, this study does not suggest an "either-or" decision between advanced fast fission energy and fusion energy.

Fast fission and fusion energy are represented in this study by corresponding nuclear reactors and the radioactive waste produced by them is compared directly. The comparison shall be done for reactors of the experimental and the commercial type, because the radioactive waste produced by these different types of reactors is expected to be different. The comparison will be provided in terms of radioactive waste parameters which characterize 
the volume and the specific and absolute activation of the waste.

\subsection{Background}

The absorption of neutrons by a given nuclide either leads to its fission or its transmutation. The proper joining of nuclides leads to their fusion. All three cases give rise to new radioactive or non-radioactive nuclides. Those nuclides represent isotopes of almost all the elements of the periodic table. The following list gives an overview over the most important elements in this context. The list is arranged according to the common chemical classification of elements $[3,5]$.

1. Alkali Metals: $L i, N a, K, R b, C s, F r$.

2. Alkaline Earth Metals: $B e, M g, C a, S r, B a, R a$.

3. Lanthanides: $L a, C e, P r, N d, P m, S m, E u, G d, T b, D y, H o, E r$, $T m, Y b, L u$; also referred to as Rare Earth Metals.

4. Actinides: $A c, T h, P a, U, N p, P u, A m, C m, B k, C f$; also referred to as Transuranics, if three elements, $A c, T h$ and $P a$, which rank before uranium, and uranium itself are excluded. The transuranics without $P u$ are referred to as Minor Actinides.

5. General Metals: $C r, M n, F e, C o, N i, C u, Y, Z r, N b, M o, T c$, $R u, C d, I n, S n, S b, T e, P b, B i$, and others.

6. Noble Metals: $R h, P d, A g, I r, P t, A u, H g$, and others.

7. Noble Gases: $H e, N e, A r, K r, X e, R n$.

It is useful to arrange the elements and their isotopes in groups, since the difference between the radioactive waste from fission and fusion reactors is characterized by its composition of those groups. Four groups are 
generally distinguished; actinides, fission products, fusion products and activation products. Fission products are generated by fission, fusion products by fusion and actinides and activation products by transmutation. Fission products, actinides and the minor part of activation products originate in fission reactor fuel and fusion products originate in fusion reactor fuel. The major part of activation products originates in fission and fusion reactor structure and coolant.

An important subset of actinides are non-easily fissile isotopes of actinides that are converted to easily fissile isotopes of actinides by transmutation. Those isotopes of actinides are called fertile isotopes.

Neutrons are necessary to maintain the immediate fission reaction in fission fuel. Hence, the generation of fission products, actinides and activation products is inherent to fission reactors. This holds true for all fission fuels. No neutrons are necessary to maintain the immediate fusion reaction in fusion fuel. Hence, only the generation of fusion products is inherent to fusion reactors. Depending on the fusion fuel, the fusion reaction may generate neutrons. The easiest to achieve, and therefore intensively pursued, fusion reaction is the one between Deuterium and Tritium ( $D-T$ reaction). It will generate neutrons and hence activation products are inherent to these fusion reactors, too. For both fission and near-term $D-T$ fusion reactors to breed their own fuel, neutrons are mandatory.

Fusion products consist entirely of hydrogen or helium, while fission products generally comprise alkali metals, alkaline earth metals, rare earth metals, general metals, noble metals and noble gases. Activation products contain an elemental range comparable to the one of fission products. However, isotopes of elements of fission products have a far higher number of neutrons than isotopes of the same elements of activation products. Therefore, fission products usually feature a far higher specific activation than activation products. While fission and fusion products are more or less the same for any fission or fusion fuel, activation products and the associ- 
ated specific activation can differ considerably with different structure and coolant used in fission and fusion reactors.

The neutron flux is within approximately one order of magnitude in almost all components of a fission reactor, but can differ by several orders of magnitude in components of a fusion reactor. The peak neutron flux in fission and fusion reactors, however, is approximately the same $\left(10^{13} \ldots 10^{15} \frac{\mathrm{n}}{\mathrm{cm}^{2} \mathrm{~s}}\right)$, although with a different energy spectrum $(0.025 \mathrm{eV} \ldots$ $10 \mathrm{MeV}$ for thermal and fast fission reactors, $0.025 \mathrm{eV} \ldots 14 \mathrm{MeV}$ for $\mathrm{D}-T$ fusion reactors).

Thus on the one hand, fission reactors produce radioactive waste with a far higher specific activation than fusion reactors do. But on the other hand, fusion reactors generate a much larger volume of radioactive waste than fission reactors do, which possibly could result in a far higher absolute activation for fusion reactors than for fission reactors.

\subsection{Focus}

The characteristic feature of a self-sufficient fuel cycle is the breeding of fuel atoms by irradiation of fertile atoms with neutrons. The breeding takes place in parallel to the power generation in a reactor. Breeding and power generation are conflicting goals, because efficient breeding requires short irradiation times, while efficient power generation requires long irradiation times. Short irradiation times allow fuel atoms to build up without being destroyed again by subsequent fission or transmutation. Long irradiation times allow to extract as much energy as possible from the fuel atoms without expensive refueling of the reactor [27].

The breeding performance of a reactor is measured in terms of its breeding ratio. It is defined as the ratio of the number of generated fuel atoms to the number of consumed fuel atoms. Apparently, in a given reactor, fuel atoms that release only few neutrons per fission or fusion reaction - 
provided the latter one generates neutrons at all - will result in a lower breeding ratio than fuel atoms that release more neutrons per respective reaction. Thus the neutron yield of the fission or corresponding fusion reaction is a decisive factor in determining the breeding ratio [27].

A breeding ratio of greater than unity means a net generation of fuel atoms, a breeding ratio of less than unity means a net consumption of fuel atoms. Note, that a breeding ratio of greater than unity does not bring about a perpetuum mobile, because now fertile atoms have to be replenished instead of fuel atoms. Fertile atoms have a greater natural abundance than fuel atoms and hence breeding only means that the resources of fuel atoms can be extended by utilizing the resources of fertile atoms. In particular, this results in a longer life-time of an energy conversion system based on fission or $(D-T)$ fusion.

Since the tritium component of $D-T$ fusion fuel is not naturally abundant, $D$ - $T$ fusion reactors operate on a self-sufficient fuel cycle by necessity. The lithium isotopes ${ }^{6} \mathrm{Li}$ and ${ }^{7} \mathrm{Li}$ serve as the fertile isotopes and the hydrogen isotope $T={ }^{3} H$ - together with the naturally abundant hydrogen isotope $D={ }^{2} H$ - serves as the fuel isotope. Fission fuel is naturally abundant, so that an immediate necessity for a self-sufficient fuel cycle does not exist. However, the natural resources of uranium, which is the major fission fuel, are relatively limited. This can be concluded from some basic considerations.

At the current rate and the current method of uranium usage in thermal fission Laght Water Reactors ( $L W R$ ), this major fission fuel will be depleted within approximately one hundred years. An average $1,000 M W_{e}=1 G W_{e}$ $L W R$ requires annual refueling equivalent to $25 t$ of enriched uranium. With natural uranium having $0.72 n u c l i d e-\%{ }^{235} U$ and enriched uranium having $3.3 n u c l i d e-\%{ }^{235} U$, the amount of natural uranium necessary to manufacture $25 \frac{t}{G W_{e} y}$ of enriched uranium is about $150 \frac{t}{G W_{c} y}$, i.e six times as high. 
Reference [33] quotes natural uranium resources recoverable at a price of less than $130 \frac{\mathrm{s}}{\mathrm{kg}}$ to be approximately 6 million tons. The current $L W R$ reactor population makes for $400 G W_{e}$, resulting in an annual natural uranium demand of $60,000 t$. That does not account for a possible increase in the reactor population, e.g. due to coal, oil and gas energy being replaced by fission energy. Hence, the natural uranium resources will last for not more than another one hundred years. The same reference cites a number of 4 billion tons of natural uranium dissolved in seawater. This could secure the supply of natural uranium for another seventy thousand years, however at a price of more than $500 \ldots 1,000 \frac{\mathrm{s}}{\mathrm{kg}}$. Moreover, possibly only $10 \%$ of the natural uranium dissolved in seawater could be recovered, i.e. the seventy thousand year supply shrinks to seven thousand years [33].

Also, approximately $350,000 t$ or $350,000 \mathrm{~m}^{3}$ seawater have to be processed to gain only $1 \mathrm{~kg}$ of natural uranium. With the above natural uranium demand of $60,000 \frac{t}{y}$, an amount of seawater equal to $2.1 \cdot 10^{13 \frac{t}{y}}$ or $665,000 \frac{\mathrm{m}^{3}}{\mathrm{~s}}$ would have to be processed - a formidable task for ion exchangers [33].

Thus, a self-sufficient fuel cycle seems to be mandatory for fission energy to continue being a major energy source beyond the point in time where fusion energy could become available. Moreover, fission reactors operating on a self-sufficient fuel cycle shall be chosen for the outlined comparison to enhance comparability of fission and fusion reactors as well as that of reactor concepts.

It is possible to operate thermal fission reactors on a self-sufficient fuel cycle. Then, the thorium isotope ${ }^{232} \mathrm{Th}$ serves as the fertile isotope and the uranium isotope ${ }^{233} U$ serves as the fuel isotope. However, ${ }^{233} U$ has a low neutron yield in the thermal energy range, so that the breeding ratio is only slightly greater than 1.0. It is easier to operate fast fission reactors on a self-sufficient fuel cycle, where advantage can be taken of the high neutron yield of the plutonium isotope ${ }^{239} \mathrm{Pu}$ in the fast energy range. Then, the 
uranium isotope ${ }^{238} \mathrm{U}$ serves as the fertile isotope and ${ }^{239} \mathrm{Pu}$ serves as the fuel isotope. The breeding ratio of fast fission reactors can be as high as 1.3. Therfore, only fast fission reactors offer a sufficient potential for breeding. In any case, if no ${ }^{233} \mathrm{U}$ or ${ }^{239} \mathrm{Pu}$ is initially available, the reactor start-up would have to be done by using ${ }^{235} U[27,56]$.

As mentioned in Section 1.1, a solution to the problem of long-term storage of radioactive waste does not yet exist. Hence, any measure that lowers the long-term hazard of radioactive waste without drawing upon a specific long-term storage method could be suitable to alleviate this problem.

In particular, it is appropriate to separate actinides from the remainder of the radioactive waste from fast fission reactors, especially since a major share of these represents potential fuel in addition to the bred ${ }^{239} \mathrm{Pu}$. This is due to the fact that most actinides become economically fissionable at high neutron energies and do no longer represent a reactor poison then. Also, without such a separation, the intended comparison would be distorted, because there is no comparably long-lived counterpart to actinides in radioactive waste from fusion reactors.

It must be noted however, that actinide separation will not be complete, since the chemical processes involved do not allow for a $100 \%$, but rather only $99.9 \%$ separation efficiency [2]. The radioactive waste from fast fission reactors will thus always retain some actinides. However, the simplifying assumption of complete separation is made for the purpose of this study. The effect of this assumption on the radioactive waste parameters and its justification is assessed in Section 5.1.1.

Finally, recent developments in fission as well as fusion reactor technology and design lend themselves to a comparison of the outlined type.

The Integral Fast Reactor (IFR) concept was launched in 1984 by the Argonne National Laboratory (ANL) [5]. It represents a self-sufficient commercial fast fission reactor.

The IFR concept is based on experiences and ideas collected in the oper- 
ation of the Experimental Breeder Reactor I $(E B R-I)$ and the self-sufficient Experimental Breeder Reactor II (EBR-II) back in the 1950s and 1960s [44]. $E B R-I I$ was to be the final step before a commercial fast fission reactor.

The Reduced Activation Ferrite Helium Tokamak (RAFHT) is a selfsufficient commercial fusion reactor proposed in 1989 by the Senior Committee on Environmental, Safety and Economy Aspects of Magnetic Fusion Energy (ESECOM) [19]. It has the highest potential for realization among other commercial fusion reactors considered by ESECOM.

The International Thermonuclear Experimental Reactor (ITER) is being developed since 1988 under the auspices of the International Atomic Energy Agency (IAEA) [23]. It represents an experimental fusion reactor that is to be the final step before a commercial fusion reactor.

The experimental nuclear reactors $E B R-I I$ and $I T E R$ have similar demonstrative functions with respect to the commercial nuclear reactors $I F R$ and $R A F H T$. EBR-II was designed to establish operation experience with and confirm the feasibility of a self-sufficient fuel cycle for a fast fission reactor. $I T E R$ roughly has the same goals for a fusion reactor. However, EBR-II could start out with a functioning fast fission reaction, while ITER first has to show that the fusion reaction can be maintained at all.

\subsection{Structure}

This study will cover only a small section of the wide field of radioactive waste. Precise definitions and limitations within this small section have to be established.

Chapter 2 provides a detailed definition of what is to be considered radioactive waste in this study and presents the definitions of and possible standards for the radioactive waste parameters to which the comparison is oriented.

Chapter 3 gives an overview of the methods applied to obtain the different radioactive waste parameters for the different nuclear reactors. This 
comes basically down to a description of the different computer codes employed to calculate the radioactive waste parameters.

Chapter 4 describes in detail the two fast fission and two fusion reactors considered in this study. The histories and objectives, the operation schedules and the designs are covered for all nuclear reactors. For fast fission reactors, additional information is given on the reprocessing of radioactive waste, especially on the separation of actinides from radioactive waste. Knowledge about those aspects is important to the correct interpretation of the comparison. For fusion reactors, information on the reprocessing of radioactive waste is not given, because as of now no appreciable reprocessing concepts exists. Notwithstanding this fact, exemplary ideas of fusion reactor radioactive waste reprocessing are given in Reference [50].

Chapter 5 presents the actual comparison, showing the radioactive waste parameters for experimental and commercial nuclear reactors and giving explanations for particular behavior modes of those parameters.

Chapter 6 completes this study with a summary and conclusion. Appendix $A$ contains various tables with detailed information necessary to understand the essence of this study. Appendix B carries tables and figures that were too numerous or extensive to be given in Chapter 5. The computer codes developed by the author of this study and all information necessary to run them are filed with the computer librarian of the Department of Nuclear Engineering of the Massachusetts Institute of Technology. Therefore, a listing of the computer codes shall be forgone here.

\subsection{Previous Studies}

Several studies have been conducted with respect to radioactive waste from fast fission and fusion reactors. The set of radioactive waste parameters chosen for those studies generally is more sparse and often different from the one chosen for this study. The same holds true for the times after discharge 
from the nuclear reactor at which the radioactive waste is characterized by those radioactive waste parameters.

Even if comparable radioactive waste parameters or times were chosen, the type of characterization of radioactive waste still is different, i.e. radioactive waste parameters are sometimes applied only to nuclides contained in radioactive waste and sometimes only to radioactive waste as an entity. Also, specific radioactive waste parameters are related to different magnitudes, like mass or volume of radioactive waste or electric power output of the nuclear reactor. Only a few of those studies compare radioactive waste from fast fission and fusion reactors directly to each other. All this leads to a lack in consistency. The studies conducted so far shall be briefly listed here.

"The EBR-II Fuel Cycle Story" [44] covers the EBR-II self-sufficient closed fuel cycle and the radioactive waste associated with it. The study "Fusion Waste Management - Safety and Environment Studies 1985-1986 -" [12] describes radioactive waste produced by the Next European Torus fusion reactor $(N E T)$. It is the most detailed study on fusion reactor radioactive waste and the results are supposed to be applicable to ITER also. The report "Safety and Environment for ITER, Records and Conclusions" $[10]$ is a misnomer for a study that considers radioactive waste from fusion reactors similar to ITER but not from ITER itself. The study "USContributions to the Homework for ITER" [11] covers radioactive waste from ITER in some detail. The "Report of the Senior Committee on Environmental, Safety and Economic Aspects of Magnetic Fusion Energy" [19] compares commercial fusion reactors and a commercial fast fission reactor and also contains some sections about associated radioactive waste. The book "Fusion and Fast Breeder Reactors" [13] extensively deals with the entire self-sufficient fuel cycle of fast fission and fusion reactors and with radioactive waste associated with it. However, it does not provide a direct comparison of radioactive waste from fast fission and fusion reactors. 


\section{Chapter 2}

\section{Radioactive Waste Parameters}

\subsection{Introduction}

$\alpha-, \beta$ - and $\gamma$-radiation emitted by radioactive nuclides, i.e. radionuclides, during their radioactive decay interact with cells of all life forms of the biosphere. This interaction can have negative or positive effects on a cell. Because of either outcome of the interaction, the exposure of the biosphere to radiation from radionuclides represents a hazard to the biosphere.

The hazard depends on the type and concentration of radionuclides in the biosphere. Natural radionuclides are in equilibrium with the biosphere and the biosphere therefore is adapted to them. For man-made radionuclides, as produced in the application of fast fission or fusion reactions, the type and concentration upset the equilibrium of the biosphere and the biosphere therefore is not adapted to them.

Equilibrium will be reached for man-made radionuclides only after their radioactive decay has occured. During the time required for this radioactive decay, radiation from man-made radionuclides should be isolated from the biosphere in order to keep the associated hazard low. Isolation encompassing radiation from man-made radionuclides implies isolation of the radionuclides themselves.

Radioactive waste contains radioactive and non-radioactive nuclides. Since separation of radioactive from non-radioactive nuclides is economi- 
cally and technically hardly feasible, all the radioactive waste has to be isolated from the biosphere, rather than just the radioactive nuclides.

Radioactive waste management can be defined as comprising the two tasks of radioactive waste handling and radioactive waste storage. Radioactive waste handling shall be understood as the sum of all activities that transport radioactive waste and convert it into, or retrieve it from, a waste form contained in a waste container. Waste form and waste container compose a waste package suitable for radioactive waste storage. The conversion might either happen directly or with previous, the retrieval directly or with subsequent reprocessing of the radioactive waste. Radioactive waste storage shall be understood as the sum of all activities that transport and store waste packages. Storage may be done temporarily (Monitored Retrievable Storage) and/or finally, depending on whether the radioactive waste requires temporary storage to establish by radioactive decay of its radionuclides conditions suitable for final storage. Between temporary and final radioactive waste storage, radioactive waste handling could be necessary.

Structural stability of a waste package is a basic prerequisite for the isolation thus devised to be effective. As a function of time, a stable waste package will deteriorate to an unstable waste package. This, however is supposed to happen in times on the order of those necessary for radionuclides to reach equilibrium. Backfilling of void spaces in waste packages and in between waste packages with grouting or equivalent material can delay (but also promote) the deterioration and increases the isolation.

Isolation of radioactive waste must be ensured both during radioactive waste handling and storage. Isolating radioactive waste rather than discarding it renders the term radioactive waste storage more appropriate than the term radioactive waste disposal and consequently the term repository is used for the corresponding radioactive waste storage facility, rather than the term dump for a radioactive waste disposal facility. 
Depending on the type and concentration of radionuclides in radioactive waste, it will be classified as low, intermediate or high level radioactive waste and the task of isolation of radioactive waste assumes different degrees of difficulty with this classification. High level radioactive waste has to be stored in deep repositories according to Part 60 of Chapter 10 of the Code of Federal Regulations (10CFR60) [48], while low and intermediate level radioactive waste may be stored in shallow repositories according to Part 61 of Chapter 10 of the Code of Federal Regulations (10CFR61) [49].

In order to determine the type and degree of isolation required, it is important to characterize radioactive waste for essentially the entire time - after discharge from the reactor - required for the radionuclides in radioactive waste to reach equilibrium. The characterization can be split up into short-term $(100 s, 1 h, 1 d, 1 w, 1 y)$, intermediate-term $(10 y, 100 y)$ and long-term $(1,000 y, 10,000 y)$ characterization. Short-term and part of the intermediate-term characterization apply to radioactive waste handling, long-term and part of the intermediate-term characterization apply to radioactive waste storage in temporary and final form.

Fast fission reactor components will undergo extensive radioactive waste handling, which already is outlined qualitatively and quantitatively [5]. Fusion reactor components will have to undergo some kind of radioactive waste handling too. However, at this point in time not even a generally accepted qualitative assessment of radioactive waste handling is available for fusion reactors [50]. For these reasons, the comparison of radioactive waste from fast fission and fusion reactors will limit itself to the comparison of reactor components as discharged from the nuclear reactor.

However, qualitative details known about actual radioactive waste handling for fast fission and fusion reactors will be incorporated to modify discharged reactor components in a way so that they reflect the status subsequent to actual radioactive waste handling; in particular, no fast fission or fusion reactor component will contain liquids or gases. Also, no fast fission 
reactor component will contain actinides, since those are ideally recycled back into the fast fission reactor $[5,44]$.

Thereby, radioactive waste in this study can roughly be set equal to the solid fraction of reactor components, possibly converted to a special waste form with the same specific activation as the original reactor component and contained in a waste container to form a waste package suitable for radioactive waste storage.

Characterization of radioactive waste in terms of its activation can be done by evaluating distinct radioactive waste parameters as outlined in subsequent sections. The radioactive waste parameters chosen in this study are volume, radioactivity, whole body $\gamma$-dose rate, decay power, Biological Hazard Potential ( $B H P)$, radioactive waste classification index and intruder dose rate of or due to radioactive waste under consideration. It should be noted that these parameters are pertinent to US regulatory standards, where applicable. Countries other than the US do generally have different regulatory standards, i.e. the results of this study might not be immediately applicable to these countries.

Radioactive waste parameters can either be specific or absolute. Specific ones are generally used to establish classifications or standards and absolute ones give a hint to the total hazard associated with radioactive waste. Specific radioactive waste parameters will always relate to the volume of radioactive waste - having dimensions "per $\mathrm{m}^{3}$ "; absolute radioactive waste parameters are derived through multiplication of the specific radioactive waste parameters by the volume of radioactive waste.

Specific radioactive waste parameters are independent of a particular waste form, or a particular waste form volume, as long as no significant dilution of radionuclides in radioactive waste takes place during radioactive waste handling. Absolute radioactive waste parameters linearly depend on a particular waste form volume. 


\subsection{Volume}

The volume of radioactive waste is important with respect to radioactive waste handling and radioactive waste storage. The dimensions of facilities for handling and storage are chiefly ruled by the dimensions of the reactor components, which for the purpose of this study constitute the radioactive waste.

Since the cost of facilities for radioactive waste management is a strong function of the size of the required shielding against radiation from radioactive waste, and the dimensions of the reactor components chiefly determine this size, their volumes have an important impact on economic considerations.

Also, a particular reactor component that ranks way below all other reactor components with respect to specific radioactive waste parameters might turn out to have the highest values of absolute radioactive waste parameters due to its large volume, and vice versa.

With the exception of liquid and gaseous fission products, the activation of liquid and gaseous constituents of reactor components is generally much lower than the one of solid constituents. Therefore, the removal of those constituents during radioactive waste handling is of minor importance to the activation. Their contribution is not accounted for in the calculation of radioactive waste parameters; in particular the effective volume of a reactor component is then given by its solid volume. In this sense, liquid and gaseous fission products represent solid constituents.

Chiefly tritium and radioactive helium are affected by the outlined simplification. Radioactive corrosion products contained in liquids or gases originate from solid constituents and therefore are accounted for.

Fast fission reactor components in this study will be approximated as a number of $N_{c}$ hollow or $N_{f}$ full cylinders or as a number of $N_{d}$ hollow or $N_{a}$ full blocks of hexagonal cross section. Hollow cylinders have an outer diameter $D_{c}$, a wall thickness $t_{c}$ and a height $H_{c}$. Full cylinders are characterized by an outer diameter $D_{f}$ and a height $H_{f}$. For the hollow 
hexagonal blocks an outer measure $S_{d}$ across the flats of the hexagon, a wall thickness $t_{d}$ and a height $H_{d}$ can be specified, while full hexagonal blocks feature an outer measure $S_{a}$ across the flats of the hexagon and a height $H_{a}$. (The indices $c, f, d, a$ stand for clad, fuel, duct and assembly). The associated (approximate) solid volumes are specified by Equations 2.1, 2.2, 2.3 and 2.4. $\eta_{a}$ represents a solid volume fraction.

$$
\begin{aligned}
V_{c} & =N_{c} \frac{\pi}{4} H_{c}\left(D_{c}^{2}-\left(D_{c}-2 t_{c}\right)^{2}\right) \\
V_{f} & =N_{f} \frac{\pi}{4} H_{f} D_{f}^{2} \\
V_{d} & =N_{d} 2 \sqrt{3} S_{d} t_{d} H_{d} \\
V_{a} & =\eta_{a} N_{a} \frac{\sqrt{3}}{2} S_{a}^{2} H_{a}
\end{aligned}
$$

Fusion reactor components $c$ in this study will be approximated as hollow cylinders of inner radius $R_{c_{i}}$, outer radius $R_{c_{o}}$ and height $H_{c}$. With $\eta_{c}$ the solid volume fraction, the solid volume of a fusion reactor component is approximated as given in Equation 2.5.

$$
V_{c}=\eta_{c} \pi H_{c}\left(R_{c_{o}}^{2}-R_{c_{i}}^{2}\right)
$$

\subsection{Radioactivity}

Radioactivity is the classical radioactive waste parameter. It should be noted, however, that radioactivity as a radioactive waste parameter itself is not a sufficient measure of the hazard associated with radioactive waste. The radioactivity of each radionuclide in radioactive waste merely gives the number of decay events for this radionuclide per time. The radioactivity of radioactive waste merely gives the number of decay events for this radioactive waste per time. Only in combination with weighting factors 
for radionuclides does the radioactivity of each radionuclide in radioactive waste and subsequently the radioactivity of radioactive waste gain some meaning. Application of such weighting factors leads to all remaining radioactive waste parameters used in this study.

Possible weighting factors for radionuclides are the whole body $\gamma$-dose released per radioactive decay, the energy released per radioactive decay, the specific radioactivity limit in radioactive waste in order not to exceed a certain whole body dose rate, the specific radioactivity limit in radioactive waste in order to establish classification of radioactive waste and the pathway dose conversion factors in order to determine actual whole body dose rates under given scenarios.

Specific radioactivities of radionuclides in radioactive waste are obtained by solving the coupled linear equation system describing the generation and destruction of radionuclides by neutron absorption and radioactive decay and multiplying the resulting concentration of all radionuclides $n$ by their decay constants $\lambda_{n}$. Once the specific radioactivity $A_{n}^{*}$ of each radionuclide in radioactive waste is known, the specific radioactivity $A_{R W}^{*}$ of radioactive waste can be determined by simple summation according to Equation 2.6. The absolute radioactivity $A_{R W}$ of radioactive waste is the result of multiplication by the radioactive waste volume $V_{R W}$ as given in Equation 2.7.

$$
\begin{gathered}
A_{R W}^{*}=\sum_{n=1}^{n_{n}} A_{n}^{*} \\
A_{R W}=A_{R W}^{*} V_{R W}
\end{gathered}
$$

\subsection{Whole Body $\gamma$-Dose Rate}

The whole body $\gamma$-dose rate at $R=1 m$ distance from radioactive waste gives an estimate of the radiation load on personnel during radioactive waste handling. At this distance, easily shielded $\alpha$ - and $\beta$-dose rates become 
unimportant and thus for activation products the whole body $\gamma$-dose rate alone is a sufficient radioactive waste parameter. For fission products and a few actinides, however, neutron emission takes place for a short time after discharge of radioactive waste from the nuclear reactor. Therefore the whole body $\gamma$-dose rate is valid as a radioactive waste parameter for fission products and actinides only for times greater than $1 d$ after discharge.

Direct contact dose rates as opposed to dose rates at a distance $R$ are generally higher because $\alpha$ - and $\beta$-dose rates have to be accounted for also.

Whole body $\gamma$-dose rates are determined for point radioactivity sources, assuming that all relevant radionuclides in radioactive waste are lumped together in one spatial point. Self-shielding due to the actual shape of radioactive waste thus is not accounted for. The $\gamma$-power $\dot{q}_{\gamma_{n}}$ of radionuclide $n$ emitted by the point source can be determined by Equation 2.8, where $A_{n}^{*}$ is the specific radioactivity of this radionuclide in radioactive waste and $E_{\gamma_{n}}$ is the energy of the $\gamma$-radiation emitted by it.

$$
\dot{q}_{\gamma_{n}}=A_{n}^{*} E_{\gamma_{n}}
$$

The resulting $\gamma$-energy flux $q_{\gamma_{n}}^{\prime \prime}$ at a distance $R$ is given by Equation 2.9, where $A=4 \pi R^{2}$ is the surface of a sphere of radius $R$.

$$
q_{\gamma_{n}}^{\prime \prime}=\frac{\dot{q}_{\gamma_{n}}}{A}
$$

Upon entry into the whole body, this $\gamma$-energy flux decays by a factor $\mu$ per entry depth $d r$, as shown in Equation 2.10.

$$
\frac{d q_{\gamma_{n}}^{\prime \prime}}{d r}=\mu q_{\gamma_{n}}^{\prime \prime}
$$

Equations 2.9 and 2.10 can be combined to give the whole body $\gamma$-dose rate as stated in Equation 2.12; $\rho, d V$ and $d m$ are the density, differential volume and differential mass of the whole body. 


$$
\begin{aligned}
& \frac{d \dot{q}_{\gamma_{n}}}{A d r}=\mu q_{\gamma_{n}}^{\prime \prime} \\
& \frac{d \dot{q}_{\gamma_{n}}}{d V}=\mu q_{\gamma_{n}}^{\prime \prime} \\
& \frac{d \dot{q}_{\gamma_{n}}}{\rho d V}=\frac{\mu}{\rho} q_{\gamma_{n}}^{\prime \prime} \\
& \frac{d \dot{q}_{\gamma_{n}}}{d m}=\frac{\mu}{\rho} q_{\gamma_{n}}^{\prime \prime}
\end{aligned}
$$

The left hand side of Equation 2.11 is just the definition of the specific whole body $\gamma$-dose rate $\dot{D}_{\gamma_{n}}^{*}$ of each radionuclide in radioactive waste and therefore Equation 2.12 holds true.

$$
\dot{D}_{\gamma_{n}}^{*}=\frac{\mu}{\rho} q_{\gamma_{n}}^{\prime \prime}=\frac{\mu}{\rho} \frac{A_{n}^{*} E_{\gamma_{n}}}{4 \pi R^{2}}
$$

Multiplication of $\dot{D}_{\gamma_{n}}^{*}$ by the quality factor of $\gamma$-radiation (i.e. $Q_{\gamma_{n}}=1$ ) results in a specific whole body $\gamma$-dose rate $\dot{H}_{\gamma_{n}}^{*}=\dot{D}_{\gamma_{n}}^{*} Q_{\gamma_{n}}$ of each radionuclide in radioactive waste.

The specific whole body $\gamma$-dose rate $\dot{H}_{\gamma_{R W}}$ of radioactive waste can be derived by simple summation as provided in Equation 2.13.

$$
\dot{H}_{\gamma_{R W}}^{*}=\sum_{n=1}^{n_{n}} \dot{H}_{\gamma_{n}}^{*}
$$

And multiplication by the volume of radioactive waste results in the absolute whole body $\gamma$-dose rate $\dot{H}_{\gamma_{R W}}$ of radioactive waste as given in Equation 2.14.

$$
\dot{H}_{\gamma_{R W}}=\dot{H}_{\gamma_{R W}}^{*} V_{R W}
$$


The resulting whole body $\gamma$-dose rates should be compared to the radiation protection standards explained in Section 2.6 in order to determine whether hands-on radioactive waste handling can be conducted or handsoff, i.e. shielded, radioactive waste handling is required. In addition, Reference [39] cites a value of $2.0 \cdot 10^{-2} \frac{\mathrm{Sv}}{h}$, reached within 50y after discharge, for short-time hands-on radioactive waste handling and $2.5 \cdot 10^{-5} \frac{\mathrm{Sv}}{h}$, reached within $100 y$ after discharge, for long-time hands-on radioactive waste handling.

\subsection{Decay power}

Decay Power is the most important radioactive waste parameter with respect to radioactive waste storage. It determines the cooling equipment that has to be provided in temporary and final radioactive waste storage. In the case of final storage it also restricts the geology of the repository to highly heat conducting material. In addition, decay power rules the packing density of radioactive waste in a repository.

Specific decay powers below $1 \frac{W}{m^{3}}$ appear to be virtually negligible [39], while $10 \frac{\mathrm{W}}{\mathrm{m}^{3}}$, reached within $50 y$ after discharge, could be a possible restricting standard [39] in terms of the maximum allowed temperature rise in the repository.

For radioactive waste handling, decay power as a radioactive waste parameter does not seem to be that important, especially if shielding against radiation has to be provided. Also, there is considerable experience with handling of intensively heat radiating components, e.g. from the steel industry.

With $E_{n}$ the decay heat of radionuclide $n$ and $A_{n}^{*}$ the specific radioactivity of this radionuclide in radioactive waste, the specific decay power $P_{n}^{*}$ of this radionuclide in radioactive waste is given by Equation 2.15 . 


$$
P_{n}^{*}=A_{n}^{*} E_{n}
$$

The specific decay power $P_{R W}^{*}$ of radioactive waste then is derived by simple summation according to Equation 2.16.

$$
P_{R W}^{*}=\sum_{n=1}^{n_{n}} P_{n}^{*}
$$

Finally the absolute decay power $P_{R W}$ of radioactive waste is obtained through multiplication by the radioactive waste volume $V_{R W}$, as shown in Equation 2.17.

$$
P_{R W}=P_{R W}^{*} V_{R W}
$$

\subsection{Biological Hazard Potential}

Part 20 of Chapter 10 of the Code of Federal Regulations (10CFR20) [47] provides standards for protection against radiation. The standards restrict the whole body dose to an individual resulting from exposure of this individual to radiation over a given time. Whole body dose standard and time can be combined to form whole body dose rate standards.

Naturally, those regulatory standards should be lower than the injury standards. Measured whole body dose rates feature an uncertainty that is presumably lower than the one for calculated whole body dose rates. However, no information was available on whether regulatory standards already consider those uncertainties or whether appropriate safety factors have to be applied to measured or calculated whole body dose rates. This study assumes the former case.

Different standards apply to areas where access is controlled - referred to as restricted areas - and areas where access is not controlled - referred to as unrestricted areas. Exposure to radiation in the former type of areas 
is equivalent to occupational exposure, exposure to radiation in the latter type of areas represents public exposure.

For restricted areas, the whole body dose rate received by an individual shall not be greater than $5 \frac{\mathrm{rem}}{\mathrm{y}}$ or $5.7 \cdot 10^{-6} \frac{\mathrm{Sv}}{\mathrm{h}}$. Under exceptional circumstances, $12 \frac{\text { rem }}{y}$ or $1.4 \cdot 10^{-5} \frac{S v}{h}$ are allowed. For unrestricted areas the whole body dose rate received by an individual shall be limited to $0.5 \frac{\mathrm{rem}}{\mathrm{y}}$ or $5.7 \cdot 10^{-7} \frac{\mathrm{Sv}}{h}$. For the purpose of $10 C F R 20$ the radiation generating radionuclides in restricted or unrestricted areas can be airborne or waterborne and can either be soluble or insoluble in air or water. Table 2.1 summarizes the resulting eight radiation environments [47].

\begin{tabular}{|l|l|l|}
\hline \hline restricted area & air & soluble \\
\cline { 3 - 3 } & & insoluble \\
\cline { 2 - 3 } & \multirow{2}{*}{ water } & soluble \\
& & insoluble \\
\hline unrestricted area & \multirow{2}{*}{ air } & soluble \\
& & insoluble \\
\cline { 3 - 3 } & water & soluble \\
& & insoluble \\
\hline
\end{tabular}

Table 2.1: Radiation Environments according to 10CFR20, Adapted from [47]

Intake by inhalation or ingestion of thus contaminated air or water to the whole body leads to a corresponding whole body dose rate. Given a rate of intake, the specific radioactivity of each radionuclide in air or water has to be limited in order for the resulting whole body dose rate to meet the 10CFR20 standards.

Those specific radioactivity limits are called Maximum Permissible Concentrations (MPCs) and are listed in 10CFR20. Table I of 10CFR20 provides $M P C$ s for exposure to radiation from airborne or waterborne radionuclides in restricted areas, Table II provides MPCs for exposure to radiation from airborne or waterborne radionuclides in unrestricted areas due to ef- 
fluents from restricted areas. MPCs are available for fusion products, fission products, actinides and activation products [47].

The $M P C_{n}$ for a certain radionuclide $n$ can be derived, if a volume intake rate $\dot{V}\left(t_{0}\right)$ of air or water by the whole body and a specific radioactivity $A_{n}^{*}\left(t_{0}\right)$ of this radionuclide in air or water at time $t_{0}$ after begin of intake are assumed. This procedure accounts for exposure to internal radiation but not for exposure to external radiation. In a time interval $d t_{0}$ at time $t_{0}$ the absolute whole body radioactivity intake rate $\dot{A}_{n}\left(t_{0}\right)$ and absolute whole body radioactivity intake $d A_{n}\left(t_{0}\right)$ are then given in Equations 2.18 and 2.19.

$$
\begin{aligned}
\dot{A}_{n}\left(t_{0}\right) & =A_{n}^{*}\left(t_{0}\right) \dot{V}\left(t_{0}\right) \\
d A_{n}\left(t_{0}\right) & =\dot{A}_{n}\left(t_{0}\right) d t_{0}
\end{aligned}
$$

This absolute whole body radioactivity intake decays according to the effective decay constant $\lambda_{e_{n}}$, i.e. the combined biological and physical decay constant. Thus, at a time $t$ greater than $t_{0}$, this absolute whole body radioactivity intake has decayed to a value $d A_{n}\left(t, t_{0}\right)$ given by Equation 2.20.

$$
d A_{n}\left(t, t_{0}\right)=d A_{n}\left(t_{0}\right) e^{-\lambda_{e_{n}}\left(t-t_{0}\right)}
$$

Since the absolute whole body radioactivity intake happens continuously, this value has to be integrated over all "intake times" $t_{0}$, from 0 to $t$, in order to derive the absolute radioactivity $A_{n}(t)$ present in the whole body at time $t$ after begin of intake. This is shown in Equation 2.21, where $A_{n}^{*}\left(t_{0}\right)$ and $\dot{V}\left(t_{0}\right)$ are assumed to be constant and equal to $A_{n}^{*}$ and $\dot{V}$, respectively.

$$
A_{n}(t)=\int_{A_{n}(t, 0)}^{A_{n}(t, t)} d A_{n}\left(t, t_{0}\right)
$$




$$
\begin{aligned}
& =\int_{A_{n}(0) e^{-\lambda_{e_{n}}}}^{A_{n}(t)} d A_{n}\left(t_{0}\right) e^{-\lambda_{e_{n}}\left(t-t_{0}\right)} \\
& =\int_{0}^{t} \dot{A}_{n}\left(t_{0}\right) e^{-\lambda_{e_{n}}\left(t-t_{0}\right)} d t_{0} \\
& =\int_{0}^{t} A_{n}^{*}\left(t_{0}\right) \dot{V}\left(t_{0}\right) e^{-\lambda_{e_{n}}\left(t-t_{0}\right)} d t_{0} \\
& =\frac{A_{n}^{*} \dot{V}}{\lambda_{e_{n}}}\left(1-e^{-\lambda_{e_{n}} t}\right)
\end{aligned}
$$

The whole body dose rate $\dot{H}_{n}(t)$ resulting from radiation due to this absolute whole body radioactivity is obtained by multiplication of the absolute radioactivity by the average product of energy $E_{n}$ and quality factor $Q$ of all radiation emitted by radionuclide $n$, divided by the mass $m$ of the whole body. This relation is given as Equation 2.22.

$$
\dot{H}_{n}(t)=\frac{A_{n}(t) E_{n} Q}{m}
$$

The average product $E_{n} Q$ depends on the energy $E_{n r}$ and quality factor $Q_{r}$ of the various types of radiation. If the probability for emission of a particular radiation $r$ is $p_{n r}$, then the average product is defined by Equation 2.23.

$$
E_{n} Q=\sum_{r=1}^{n_{r}} p_{n r} E_{n r} Q_{r}
$$

Substituting for $A_{n}(t)$ in Equation 2.22 and resolving for $A_{n}^{*}$ yields Equation 2.24

$$
A_{n}^{*}=\frac{m \dot{H}_{n}(t) \lambda_{e_{n}}}{\dot{V} E_{n} Q\left(1-e^{-\lambda_{e_{n}} t}\right)}
$$

Now setting $\dot{H}_{n}(t)$ at a time $t$ after begin of intake equal to a 10CFR20 standard will result in a corresponding specific radioactivity $A_{n}^{*}$ of the ra- 
dionuclide in air or water, which is the desired Maximum Permissible Concentration $M P C_{n}$.

Specifically for the airborne radiation environment in restricted areas, the time $t$ is defined as 13 weeks at $40 \frac{h}{\text { week }}$, with $\dot{V}$ equal to the normal breathing rate. For the other radiation environments, certain schemes of water and air intake by the individual have to be defined also [47].

In order for the whole body dose rate resulting from exposure to the radiation of more than one radionuclide to meet the standards, the sum of the ratios of specific radioactivity $A_{n}^{*}$ in air or water and $M P C_{n}$ of all radionuclides $n$, referred to as specific Biological Hazard Potential (BHP'), must be less than unity [47]. The corresponding formula is given in Equation 2.25.

$$
B H P^{*}=\sum_{n=1}^{n_{n}} \frac{A_{n}^{*}}{M P C_{n}} \leq 1.0
$$

If $B H P^{*}$ exceeds unity, then the sum of the ratios of absolute radioactivity $A_{n}$ in an air or water volume $V$ and $M P C_{n}$ of all radionuclides $n$, referred to as absolute Biological Hazard Potential (BHP), gives the air or water volume that is required to dilute the specific radioactivities so that $B H P^{*}$ becomes equal to unity [47]. This can be cast in formulas as given in Equations 2.26, 2.27 and 2.28.

$$
\begin{aligned}
B H P & =\sum_{n=1}^{n_{n}} \frac{A_{n}}{M P C_{n}} \\
A_{n} & =A_{n}^{*} V \\
B H P & =B H P^{*} V
\end{aligned}
$$

For application to radioactive waste management, the common assumption is made that the radionuclides in radioactive waste find access to air or water in a way that establishes a specific radioactivity in air or water equal to that in the radioactive waste [13]. The specific $B H P_{R W}^{*}$ then is 
defined as in Equation 2.25. However, to derive the absolute $B H P_{R W}$, the volume $V$ of air or water in Equations 2.27 and 2.28 must be replaced by the volume $V_{R W}$ of radioactive waste.

The definition of the specific and absolute $B H P$ is applicable to radioactive waste handling and radioactive waste storage. However, the above assumption of the specific radioactivity of radionuclides being equal in radioactive waste and in air or water is time dependent and based on highly unlikely events, as can be demonstrated for the radioactive waste storage application.

For radioactive waste storage, only the exposure to radiation from waterborne radionuclides in unrestricted areas is of interest, since radioactive nuclides will find their way out of a repository as a restricted area most likely as effluents via release to the groundwater. Furthermore, in order to stay on the conservative side, solubility of the released radionuclides in water is assumed. Insoluble radioactive nuclides are much more harmless, since they are easily filtered out and precipitated during groundwater transport processes.

In the first place, repositories are not allowed to be built near the groundwater table, which makes groundwater ingression to radioactive waste highly unlikely [49]. But even for the case that groundwater ingression should occur, there are other factors which prove the above assumption to be too conservative.

Waste containers of waste packages prevent groundwater access to radioactive waste in a particular waste form for at least the time it takes for the waste container to significantly deteriorate. Thereafter, groundwater can get into direct contact with radioactive waste in a particular waste form. But even then the groundwater will be able to release only a small fraction of radionuclides in a given time.

Investigations [36] have shown, and 10CFR60 [48] for deep repositories requires, that the average fraction release rate of radionuclides from radioactive waste in a corresponding waste form to contacting groundwater 
is on the order of $\dot{f}_{R W G W}=10^{-5} \frac{1}{y}$. If radioactive waste of a given volume $I_{R W}^{\prime}$, specific radioactivity $A_{R W}^{*}$ or absolute radioactivity $A_{R W}$ and waste form is exposed to groundwater, the maximum allowed groundwater volume flow $\dot{V}_{G W}$ in order to reach a groundwater specific radioactivity $A_{G W}^{*}$ equal to that of radioactive waste can be estimated according to Equations 2.29, $2.30,2.31$ and 2.32 .

$$
\begin{aligned}
A_{R W} & =A_{R W}^{*} V_{R W} \\
\dot{A}_{G W} & =\dot{f}_{R W G W} A_{R W} \\
A_{G W}^{*} & =\frac{\dot{A}_{G W}}{\dot{V}_{G W}} \\
& =\frac{\dot{f}_{R W G W} A_{R W}}{\dot{V}_{G W}} \\
& =\frac{\dot{f}_{R W G W} A_{R W}^{*} V_{R W}}{\dot{V}_{G W}}
\end{aligned}
$$

Since it is assumed that $A_{G W}^{*}=A_{R W}^{*}$, dropping those specific radioactivities in Equation 2.31 and rearranging then yields Equation 2.32.

$$
\dot{V}_{G W}=\dot{f}_{R W G W} V_{R W}
$$

With a radioactive waste volume on the order of $V_{R W}=10 \mathrm{~m}^{3}$ this would be equivalent to a groundwater volume flow of $\dot{V}_{G W}=10^{-4} \frac{\mathrm{m}^{3}}{\mathrm{y}}$, which without question will be exceeded by an actual groundwater volume flow in the case of groundwater ingression. Assuming an actual groundwater volume flow of $1 \frac{m^{3}}{y}$ under the above release rate, the specific radioactivity of radioactive waste would be diluted by a factor of at least $10^{-4}$. The assumed groundwater volume flow is rather on the conservative side, because Reference [6] cites a number of $37 \frac{m^{3}}{y}$ per meter of groundwater flow width for 
New Mexcio; waste packages will in deed have dimensions on the order of $1 \mathrm{~m}$, so that an additional dilution by a factor of $10^{-1} \ldots 2.5 \cdot 10^{-2}$ seems likely.

Even if a specific radioactivity of water equal to that of radioactive waste would be reached locally at the waste package with deteriorated waste container, it will be naturally diluted and filtered while the groundwater travels from the restricted to the unrestricted area.

Due to the relatively long groundwater travel times from restricted to unrestricted areas, the time delay will lead to significant decay of most radionuclides so that their original specific radioactivities in radioactive waste do not hold true. 10CFR61 [48] for deep repositories requires corresponding groundwater travel times of more than $1,000 y$.

Despite those caveats, the $B H P$ is a handy waste parameter describing the worst, though extremely unlikely, case that could happen. A much more realistic estimate of the actual hazard can be achieved, if $B H P_{R W}^{*}$ and $B H P_{R W}$ as calculated are multiplied by e.g. a factor of $10^{-4}$ or less, equivalent to the above outlined dilution effect.

\subsection{Radioactive Waste Classification}

\subsubsection{Low, Intermediate and High Level Radioactive Waste}

A radionuclide can be clearly identified by the half life of its radioactive decay and the radiation that is emitted by it during this radioactive decay. Radioactive waste containing an equal concentration of radionuclides with short, medium or long half lives is characterized by a high, intermediate or low specific radioactivity. However, the short-lived radionuclides might emit relatively harmless radiation, while the long-lived radionuclides might emit extremely harmful radiation.

Also, on the one hand, a low concentration of short-lived radionuclides 
can result in a low specific radioactivity of radioactive waste and thereby decrease the effect of the associated radiation. On the other hand a high concentration of long-lived radionuclides can result in a high specific radioactivity of radioactive waste and thereby increase the effect of the associated radiation.

This shows that a classification of radioactive waste into low, intermediate and high level radioactive waste based on the half life of each radionuclide contained in it alone is not sufficient. A proper classification always requires the identification of each radionuclide by its half life and emitted radiation and the concentration of this radionuclide in radioactive waste. The natural combination of half life and concentration is the specific radioactivity of each radionuclide in radioactive waste. The emitted radiation can then be accounted for by limiting this specific radioactivity of each radionuclide in radioactive waste in order for the radioactive waste to belong to a certain class. The essence of these considerations also holds true for the MPCs as defined in Section 2.6.

Typically, radionuclides of fusion and fission products feature short half lives and $\beta$ - and $\gamma$-radiation, those of activation products feature medium half lives and $\beta$ - and $\gamma$-radiation, and those of actinides feature long half lives and $\alpha$-radiation. However, radionuclides of all four nuclide groups can have overlapping half lives and radiation emissions.

A classification of radioactive waste into low, intermediate and high level radioactive waste based solely on its specific radioactivity without identifying the radionuclides contained in it could not account for the radiation emitted by each radionuclide. Any limit for the specific radioactivity of radioactive waste must recognize this fact and therefore presumably constitutes a conservative approach to radioactive waste classification. However, this approach has been chosen, e.g. by the government of the Federal Republic of Germany, where any radioactive waste with a specific radioactivity greater than $10^{4} \frac{\mathrm{Ci}}{\mathrm{m}^{3}}$ is considered as high level radioactive waste.

Sufficient dilution of radioactive waste composed of any radionuclides 
will lead to a classification as low level radioactive waste. The limits to this procedure are set by economic constraints in terms of available storage facilities and possible surveillance.

The outlined reasoning leads to the classification of radioactive waste containing actinides as high level radioactive waste; the fact that this class of radioactive waste has to be stored in deep repositories is important with respect to proliferation and chemical toxicity issues. Also, radioactive waste containing fission products will most probably be classified as high level radioactive waste. Radioactive waste composed of activation products could either be intermediate or high level radioactive waste. In addition, for each of the above radioactive waste classes, gaseous, liquid and solid radioactive waste should be discerned, since the dilution of radionuclides increases and the specific radioactivity decreases from solid to liquid to gaseous radioactive waste.

\subsubsection{Class A, Class B, Class $C$ and Class D Ra- dioactive Waste}

Deep repositories are characterized by expensive exploration, construction and operation activities, while shallow repositories are relatively inexpensive to explore, construct and operate. However, deep repositories offer a much higher degree of isolation than shallow repositories do, which are susceptive to a violation of isolation. The most important violation of isolation in a shallow repository occurs in the case of inadvertent intrusion, where an inadvertent intruder might get direct contact with radioactive waste.

However, institutional control of access to a shallow (and deep) repository can be assumed to be effective for about $100 y$ after begin of storage, during which period inadvertent intrusion can be virtually excluded [49].

Thus, low and intermediate level radioactive waste with radionuclides that decay to approximate equilibrium conditions in less than $100 y$ does not require special protection against inadvertent intrusion. Low and interme- 
diate level radioactive waste with radionuclides that need more than $100 y$ to decay to approximate equilibrium conditions, however, does require such a special protection. For this distinction to make sense, begin of storage is assumed to take place within $5 y$ after discharge of radioactive waste from the nuclear reactor.

Although all radioactive waste consisting of radionuclides with equilibration times greater than $100 y$ has to be stored in solid form, or more specifically with less than $1.0 v o l u m e-\%$ of liquid [49], it is desirable to store some of the low or intermediate level radioactive waste containing radionuclides with equilibration times less than $100 y$ in liquid form, since this obliviates the process of solidification of the already liquid radioactive waste.

Hence, as provided in 10CFR61, low and intermediate level radioactive waste may be further divided into three classes corresponding to the radionuclides contained in it and their specific radioactivites in radioactive waste. With increasing hazard level, these classes are termed $A, B$ and $C$ [49].

Class $A$ radioactive waste may be liquid or solid, while class $B$ radioactive waste is always solid. The radionuclides in both class $A$ and $B$ radioactive waste decay to levels comparable to equilibrium conditions within the $100 y$ of institutional control. It therefore does not demand special protection against inadvertent intrusion [49].

Class $C$ radioactive waste is always solid but contains radionuclides that decay to levels comparable to equilibrium conditions only within 500y. For this time period a shallow repository with class $C$ radioactive waste must be equipped with an intruder barrier. This can be a sufficiently thick cover layer equivalent to storage at greater depth than class $A$ and $B$ radioactive waste and at least of $5 m$ thickness. It also can be an engineered structure to inhibit contact between the inadvertent intruder and the radioactive waste. The intruder barrier must be designed to have a life time of at least $500 y$. This requires that the shallow repository characteristics be evaluated for 
$500 y[49]$.

If Class $A$ radioactive waste is liquid it has to be separated from class $B$ and $C$ radioactive waste in order for the former one not to cause deterioration of the latter two by chemical interaction. Class $B$ and $C$ radioactive waste packages must be stable for at least $300 y$. The stability serves not only to ensure isolation of radionuclides but the associated perceptibility is important in the case of inadvertent intrusion [49].

For the purpose of radioactive waste classification, 10CFR61 lists Allowable Isotope Concentrations ( $A I C$ s), i.e. specific radioactivities in radioactive waste for several radionuclides and the three classes $A, B, C$. In order for radioactive waste to belong to either class $A, B$ or $C$, the sum of the ratios of specific radioactivity $A_{n}^{*}$ in radioactive waste and $A I C_{n}$ for either class of all radionuclides $n$, referred to as Radioactive Waste Class (RWC) index, must be less than unity. If the $R W C$ index exceeds unity even for class $C$, then the respective radioactive waste is no low or intermediate level radioactive waste anymore [49]. However, it does not yet necessarily have to be considered high level radioactive waste either. The corresponding formulas to determine $R W C$ indices are given as Equations 2.33, 2.34, 2.35, $2.36,2.37$ and 2.38 .

$$
\begin{aligned}
& R W C_{A}=\sum_{n=1}^{n_{n}} \frac{A_{n}^{*}}{A I C_{n_{A}}} \\
& R W C_{B} \doteq \sum_{n=1}^{n_{n}} \frac{A_{n}^{*}}{A I C_{n_{B}}} \\
& R W C_{C}=\sum_{n=1}^{n_{n}} \frac{A_{n}^{*}}{A I C_{n_{C}}} \\
& \text { Class } A: R W C_{A} \leq 1.0 \\
& \text { Class } B: R W C_{B} \leq 1.0
\end{aligned}
$$




$$
\text { Class } C: R W C_{C} \leq 1.0
$$

$A I C s$ are determined such that the whole body dose rate to an inadvertent intruder never exceeds the standards specified in 10CFR20. Specific radioactivities of radionuclides in radioactive waste translate to whole body dose rates via particular barrier and conversion factors. According to Reference [8], the AICs listed in 10CFR61 are based on partly wrong conversion factors. Therefore, this study uses AICs based on corrected conversion factors as provided in Reference [8]. This reference also exhibits a greater consistency in terms of the radionuclides for which AICs are prepared.

In addition, Reference [8] specifies another class of radioactive waste, termed $D$. This additional class is essentially the same as class $C$, with the exception, that the waste form of radioactive waste is considered to be purely metallic. The $R W C$ index for this class is determined by Equations 2.39 and 2.40 .

$$
\begin{gathered}
R W C_{D}=\sum_{i=1}^{n_{n}} \frac{A_{n}^{*}}{A I C_{n_{D}}} \\
\text { Class } D: R W C_{D} \leq 1.0
\end{gathered}
$$

\subsection{Intruder Dose Rate}

An inadvertent intruder might engage in construction or agriculture activities directly above a shallow or deep repository after institutional control ceased to be effective. This inadvertent intruder then could be unknowingly exposed to radiation originating in the repository [49]. This could either be radiation directly from radionuclides in the repository or radiation from radionuclides released from the repository.

In the case of construction activities, referred to as construction scenario, released radionuclides concentrate in air and soil above the repository. In 
the case of agriculture activities, referred to as agriculture scenario, released radionuclides concentrate in air, soil and food which has been cultivated above the repository. The inadvertent intruder will receive a whole body dose rate upon inhalation of or direct contact to air, direct contact to soil and ingestion of or direct contact to food [8]. The radiation sources air, soil and food are referred to as pathways. The point in time at which inadvertent intrusion under either scenario occurs is referred to as the beginning of the scenario.

For both shallow and deep repositories as initially restricted areas it must be proven that an inadvertent intruder under both scenarios will never receive whole body dose rates in excess of the standards specified in 10CFR20. After institutional control ceases to be effective, restricted areas become unrestricted areas for the purpose of 10CFR20 [47].

Shallow repositories will be located not more than $30 \mathrm{~m}$ below the earth's surface, while deep repositories will be located on the order of $1,000 \mathrm{~m}$ below the earth's surface and in a special geology. Even though shallow repositories only store low and intermediate level radioactive waste and deep repositories store high level radioactive waste, the hazard to an inadvertent intruder to a shallow repository will be significantly higher than the one to an inadvertent intruder to a deep repository, because of the inherently higher degree of isolation provided by the latter type of repositories. Therefore in this study, inadvertent intruder whole body dose rates shall apply only to shallow repositories, as also stated in $10 C F R 61[48,49]$.

For shallow repositories in compliance with 10CFR61 and as outlined in Reference [8], specific radioactivities of radionuclides in radioactive waste convert to specific radioactivities in air, soil or food via barrier factors. These are time delay barrier factor, site design barrier factor, site selection barrier factor and waste form barrier factor. Each of these four barrier factors is the product of auxiliary barrier factors of corresponding names. Once specific radioactivities in air, soil or food are determined, they can be 
converted to whole body dose rates via Pathway Dose Conversion ( $P D C)$ factors [8]. Due to errors in the computation of PDC factors in 10CFR61, corrected $P D C$ factors as listed in Reference [8] will be used.

The time delay barrier factor accounts for the decay of radionuclides from an initial specific radioactivity to a specific radioactivity at a later point in time [8]. However, in this study, specific radioactivities of radionuclides in radioactive waste are already calculated for all points in time under consideration. Therefore the time delay factor is not applicable and is always set equal to unity.

The site design barrier factor accounts for the design of the shallow repository. It allows specifying whether stacked or random storage of waste packages is used, whether grouting for backfilling of void spaces is utilized, and whether and what type of a special repository has been chosen. Hence it determines the degree of isolation the repository is able to provide [8].

The waste form barrier factor accounts for the extent to which waste packages are able to prevent release of radionuclides. It allows specifying the degree of prevention of dispersion of radionuclides in air, soil or food and whether a high metal content in radioactive waste significantly inhibits radionuclide transport out of it [8].

The site selection barrier factor accounts for the mode in which an inadvertent intruder will be exposed to radiation originating from the repository. This includes the repository environment and also allows specifying after what time the inadvertent intruder recognizes the exposure to radiation, depending on whether the waste packages are stable or unstable at the time of inadvertent intrusion [8].

The $P D C$, barrier and auxiliary barrier factors used in this study are summarized in Table 2.2.

For shallow repositories 10CFR 61 defines $A I C$ s which, when compared to the specific radioactivities of radionuclides in radioactive waste, serve to establish a classification of low and intermediate level radioactive waste into 


\begin{tabular}{|c|c|c|}
\hline Factor & Meaning & Dimension \\
\hline$f_{P D C_{n p}}$ & $\begin{array}{l}=\text { pathway dose conversion factor for } \\
\text { radionuclide } \mathrm{n} \text { and pathway } \mathrm{p} \\
(p=1,2,3=a i r, \text { soil }, \text { food })\end{array}$ & {$\left[\frac{S_{v} / h}{B q / m^{3}}\right]$} \\
\hline$f_{\text {onp }}$ & $\begin{array}{l}=\text { time delay barrier factor } \\
\text { for radionuclide } \mathrm{n} \text { and pathway } \mathrm{p}, \\
\quad(p=1,2,3)\end{array}$ & [1] \\
\hline$f_{d_{n p}}$ & $\begin{array}{l}=\text { site design barrier factor } \\
\text { for radionuclide } \mathrm{n} \text { and pathway } \mathrm{p}, \\
(p=1,2,3)\end{array}$ & {$[1]$} \\
\hline$f_{s_{n p}}$ & $\begin{array}{l}=\text { site selection barrier factor } \\
\text { for radionuclide } \mathrm{n} \text { and pathway } \mathrm{p}, \\
(p=1,2,3)\end{array}$ & {$[1]$} \\
\hline$f_{w_{n p}}$ & $\begin{array}{l}=\text { waste form barrier factor } \\
\text { for radionuclide } \mathrm{n} \text { and pathway } \mathrm{p}, \\
(p=1,2,3)\end{array}$ & [1] \\
\hline$\overline{f_{o_{n p j}}}$ & $\begin{aligned}= & \text { time delay auxiliary barrier factor } \mathrm{j} \\
& \text { for radionuclide } \mathrm{n} \text { and pathway } \mathrm{p}, \\
& (p=1,2,3)\end{aligned}$ & {$[1]$} \\
\hline$f_{d_{n p j}}$ & $\begin{array}{l}=\text { site design auxiliary barrier factor } \mathrm{j} \\
\text { for radionuclide } \mathrm{n} \text { and pathway } \mathrm{p}, \\
(p=1,2,3)\end{array}$ & [1] \\
\hline$f_{s_{n p j}}$ & $\begin{array}{l}=\text { site selection auxiliary barrier factor } \mathrm{j} \\
\text { for radionuclide } \mathrm{n} \text { and pathway } \mathrm{p}, \\
(p=1,2,3)\end{array}$ & {$[1]$} \\
\hline$f_{w_{n p j}}$ & $\begin{array}{l}=\text { waste form auxiliary barrier factor } \mathrm{j} \\
\text { for radionuclide } \mathrm{n} \text { and pathway } \mathrm{p}, \\
(p=1,2,3)\end{array}$ & [1] \\
\hline$f_{s d g_{n}}$ & $=$ shielding equivalent to $1 \mathrm{~m}$ of soil & [1] \\
\hline
\end{tabular}

Table 2.2: Meaning and Dimension of $P D C$, Barrier and Auxiliary Barrier Factors, Adapted from [8] 
class $A, B$ or $C$. $A I C$ s in combination with barrier factors and $P D C$ factors for all three classes, provided in supporting documents to 10CFR61 and in Reference [8], result in absolute whole body dose rates to an inadvertent intruder that fulfill the standards set in 10CFR20. Specific radioactivities of radionuclides in radioactive waste in combination with barrier factors and $P D C$ factors for all three classes result in absolute whole body dose rates to an inadvertent intruder that either fall short of or exceed the standards set in 10CFR20, depending on whether radioactive waste was classified as class $A, B$ or $C$ low or intermediate level radioactive waste or as high level radioactive waste.

The whole body dose rate to an inadvertent intruder depends on the time at which the inadvertent intrusion occurs. This time usually is measured after institutional control ceases to exist. If the beginning of storage takes place within $5 y$ after discharge of radioactive waste from the nuclear reactor, then the inadvertent intrusion could occur no more than roughly $100 y$ later. This relatively early inadvertent intrusion yields conservative intruder dose rates, in contrast to a later inadvertent intrusion. Intruder dose rates are relatively meaningless for times during institutional control, but nevertheless give an approximation of the radiation level above the shallow repository for those times.

\subsubsection{Construction Scenario}

The whole body dose rate to an inadvertent intruder under the construction scenario is based on the air and soil pathways. The time it takes to effectively engage in construction likens the time it takes to recognize waste packages. It therefore is important to specify whether waste packages are stable or unstable at the beginning of the scenario, because the recognition time is a function thereof. Also, the time after which stable waste packages deteriorate into unstable waste packages is a function of the shallow repository design [8]. In applying barrier factors and $P D C$ factors to spe- 
cific radioactivities $A_{n}^{*}$ of radionuclides $n$ in radioactive waste, the absolute whole body dose rate $\dot{H}_{R W}$ of radioactive waste can then be determined as in Equation 2.41 [8].

$$
\begin{aligned}
\dot{H}_{R W}= & \sum_{n=1}^{n_{n}} \sum_{p=1}^{n_{p}} \dot{H}_{n p} \\
= & \sum_{n=1}^{n_{n}} \sum_{p=1}^{n_{p}} f_{P D C_{n p}} A_{n p}^{*} \\
= & \sum_{n=1}^{n_{n}} \sum_{p=1}^{n_{p}} f_{P D C_{n p}} f_{o_{n p}} f_{d_{n p}} f_{s_{n p}} f_{w_{n p}} A_{n}^{*} \\
= & \sum_{n=1}^{n_{n}} f_{P D C_{n 1}} f_{o_{n 1}} f_{d_{n 1}} f_{s_{n 1}} f_{w_{n 1}} A_{n}^{*} \\
& +\quad f_{P D C_{n 2}} f_{o_{n 2}} f_{d_{n 2}} f_{s_{n 2}} f_{w_{n 2}} A_{n}^{*}
\end{aligned}
$$

Barrier factors are composed of auxiliary barrier factors as reflected in Table $2.3[8]$.

\begin{tabular}{|l|l|l|}
\hline \hline & Pathway 1 = air & Pathway 2 = soil \\
\hline$f_{o}$ & $f_{o_{n 1}}=f_{o_{n 11}}$ & $f_{o_{n 2}}=f_{o_{n 21}}$ \\
$f_{d}$ & $f_{d_{n 1}}=f_{d_{n 11}} f_{d_{n 12}} f_{d_{n 13}}$ & $f_{d_{n 2}}=f_{n_{n 21}} f_{d_{n 22}} f_{d_{n 23}}$ \\
$f_{w}$ & $f_{w_{n 1}}=f_{w_{n 11}} f_{w_{n 12}}$ & $f_{w_{n 2}}=f_{w_{n 21}}$ \\
$f_{s}$ & $f_{s_{n 1}}=f_{s_{n 11}} f_{s_{n 12}}$ & $f_{s_{n 2}}=f_{s_{n 21}} f_{s_{n 22}}$ \\
\hline
\end{tabular}

Table 2.3: Barrier and Auxiliary Barrier Factors, Construction Scenario

Auxiliary barrier factors for the time delay barrier factor are defined by Equations 2.42 and $2.43[8]$, where $\lambda_{j}, \lambda_{k}$ and $\lambda_{l}$ are decay constants of corresponding radionuclides $j, k, l$. In this study, they generally assume a value of unity.

$$
f_{\mathrm{o}_{11}}=\prod_{j=1}^{n} \lambda_{j} \sum_{k=0}^{n} \frac{\mathrm{e}^{-\lambda_{k} t}}{\prod_{l \neq k}\left(\lambda_{l}-\lambda_{k}\right)}
$$




$$
f_{o_{n 21}}=\quad f_{o_{n 11}}
$$

Auxiliary barrier factors for the site design barrier factor are defined by Equations 2.44, 2.45, 2.46, 2.47, 2.48 and 2.49. Shallow repositories with layered waste design allow stable waste packages for $500 y$, but with hot waste design allow stable waste packages for $1,000 y$ after beginning of storage. Shallow repositories with no particular design allow stable waste packages only for the time institutional control is effective [8].

$$
\begin{aligned}
f_{d_{n 11}}= & 0.75 \text { with stacked disposal } \\
& 0.50 \text { with random disposal } \\
f_{d_{n 12}=}= & 0.1 \text { with grouting } \\
& 1.0 \text { without grouting } \\
f_{d_{n 13}=} & 1.0 \text { with no particular design or unstable waste package } \\
& 0.1 \text { with layered waste design and stable waste package } \\
& 0.01 \text { with hot waste design and stable waste package }(2.46) \\
f_{d_{n 21}=} & f_{d_{n 11}} \\
f_{d_{n 22}=} & f_{d_{n 12}} \\
f_{d_{n 23}=} & 1.0 \text { with no particular design or unstable waste package } \\
& f_{s d g_{n}} \text { with layered waste design and stable waste package } \\
& f_{s d_{n}}^{2} \text { with hot waste design and stable waste package }
\end{aligned}
$$

Auxiliary barrier factors for the waste form barrier factor are defined by Equations 2.50, 2.51 and 2.52 [8].

$$
\begin{aligned}
& f_{w_{n 11}}=0.1,0.01,0.001 \text { with prevention of dispersion } \\
& 1.0 \quad \text { without prevention of dispersion } \\
& f_{w_{n 12}}=0.1,0.01 \quad \text { with transport inhibition }
\end{aligned}
$$




$$
f_{w_{n 21}}=\quad \begin{array}{ll}
1.0 & \text { without transport inhibition } \\
0.8 & \text { with low metal content } \\
0.1 & \text { with high metal content }
\end{array}
$$

Auxiliary barrier factors for the site selection barrier factor are defined by Equations 2.53, 2.54, 2.55 and 2.56 [8].

$$
\begin{aligned}
& f_{s_{n 11}}=2.01 \cdot 10^{-11} \\
& f_{s_{n 12}}=\quad 1.0 \quad \text { with unstable waste package } \\
& \frac{6}{500} \quad \text { with stable waste package } \\
& f_{s_{n 21}}=\quad \frac{500}{8780} \\
& f_{s_{n 22}}=f_{s_{n 12}}
\end{aligned}
$$

Unstable waste packages will be recognized only after $500 h$, while stable waste packages will be recognized after $6 h$. The auxiliary barrier factor $f_{s_{n 12}}=1.0$ or $f_{s_{n 12}}=\frac{6}{500}$ results from this assumption [8].

The auxiliary barrier factors used in this study are given in Table 2.4.

\begin{tabular}{|ll|}
\hline \hline Factor & Value \\
\hline$f_{\text {on }_{11}}$ & $=1.0$ \\
$f_{d_{n 11}}$ & $=0.75$ \\
$f_{d_{n 12}}$ & $=0.1$ \\
$f_{d_{n 13}}$ & $=0.1$ \\
$f_{d_{n 23}}$ & $=f_{s} g_{n}$ \\
$f_{w_{n 11}}$ & $=0.1$ \\
$f_{w_{n 12}}$ & $=0.1$ \\
$f_{w_{n 21}}$ & $=0.1$ \\
\hline
\end{tabular}

Table 2.4: Auxiliary Barrier Factors used in this Study, Construction Scenario 


\subsubsection{Agriculture Scenario}

The whole body dose rate to an inadvertent intruder under the agriculture scenario is based on the air, soil and food pathways. The time it takes to effectively engage in agriculture exceeds the time it takes to recognize waste packages. It therefore is not important to specify whether waste packages are stable or unstable at the beginning of the scenario, because the recognition time is no longer a function thereof once the engagement in agriculture proceeded far enough. Also, the time after which stable waste packages deteriorate into unstable waste packages is of no importance anymore and hence the distinction among different shallow repository designs does no longer matter [8]. In applying barrier factors and $P D C$ factors to specific radioactivities $A_{n}^{*}$ of radionuclides $n$ in radioactive waste, the absolute whole body dose rate $\dot{H}_{R W}$ of radioactive waste can then be determined as in Equation 2.57 [8].

$$
\begin{aligned}
\dot{H}_{R W}= & \sum_{n=1}^{n_{n}} \sum_{p=1}^{n_{p}} \dot{H}_{n p} \\
= & \sum_{n=1}^{n_{n}} \sum_{p=1}^{n_{p}} f_{P D C_{n p}} A_{n p}^{*} \\
= & \sum_{n=1}^{n_{n}} \sum_{p=1}^{n_{p}} f_{P D C_{n p}} f_{o_{n p}} f_{d_{n p}} f_{s_{n p}} f_{w_{n p}} A_{n}^{*} \\
= & \sum_{n=1}^{n_{n}} f_{P D C_{n 1}} f_{o_{n 1}} f_{d_{n 1}} f_{s_{n 1}} f_{w_{n 1}} A_{n}^{*} \\
& +\quad f_{P D C_{n 2}} f_{o_{n 2}} f_{d_{n 2}} f_{s_{n 2}} f_{w_{n 2}} A_{n}^{*} \\
& +\quad f_{P D C_{n 3}} f_{o_{n 3}} f_{d_{n 3}} f_{s_{n 3}} f_{w_{n 3}} A_{n}^{*}
\end{aligned}
$$

Barrier factors are composed of auxiliary barrier factors as reflected in Table 2.5 [8]. 


\begin{tabular}{|c|l|l|l|}
\hline \hline & Pathway 1 = air & Pathway 2 = soil & Pathway 3 = food \\
\hline$f_{0}$ & $f_{o_{n 1}}=f_{o_{n 11}}$ & $f_{o_{n 2}}=f_{o_{n 21}}$ & $f_{o_{n 3}}=f_{o_{n 31}}$ \\
$f_{d}$ & $f_{d_{n 1}}=\frac{1}{4} f_{d_{n 11}} f_{d_{n 12}}$ & $f_{d_{n 2}}=\frac{1}{4} f_{d_{n 21}} f_{d_{n 22}}$ & $f_{d_{n 3}}=\frac{1}{4} f_{d_{n 31}} f_{d_{n 32}}$ \\
$f_{w}$ & $f_{w_{n 1}}=f_{w_{n 11}} f_{w_{n 12}}$ & $f_{w_{n 2}}=f_{w_{n 21}} f_{w_{n 22}} f_{w_{n 23}} f_{w_{n 24}}$ & $f_{w_{n 3}}=f_{w_{n 31}}$ \\
$f_{s}$ & $f_{s_{n 1}}=f_{s_{n 11}}$ & $f_{s_{n 2}}=f_{s_{n 21}}$ & $f_{s_{n 3}}=f_{s_{n 31}}$ \\
\hline
\end{tabular}

Table 2.5: Barrier and Auxiliary Barrier Factors, Agriculture Scenario

Auxiliary barrier factors for the time delay barrier factor are defined by Equations 2.58, 2.59 and 2.60 [8], where $\lambda_{j}, \lambda_{k}$ and $\lambda_{l}$ are decay constants of corresponding radionuclides $j, k, l$. In this study, they generally assume a value of unity.

$$
\begin{array}{lc}
f_{o_{n 11}}= & \prod_{j=1}^{n} \lambda_{j} \sum_{k=0}^{n} \frac{e^{-\lambda_{k} t}}{\prod_{l \neq k}\left(\lambda_{l}-\lambda_{k}\right)} \\
f_{o_{n 21}}= & f_{o_{n 11}} \\
f_{o_{n 31}}= & f_{o_{n 11}}
\end{array}
$$

Auxiliary barrier factors for the site design barrier factor are defined by Equations 2.61, 2.62, 2.63, 2.64, 2.65 and 2.66 [8].

$$
\begin{aligned}
f_{d_{n 11}=} & 0.75 \text { with stacked storage } \\
& 0.50 \text { with random storage } \\
f_{d_{n 12}=} & 0.1 \quad \text { with grouting } \\
& 1.0 \text { without grouting } \\
f_{d_{n 21}=}= & f_{d_{n 11}} \\
f_{d_{n 22}=}= & f_{d_{n 12}} \\
f_{d_{n 31}=}= & f_{d_{n 11}} \\
f_{d_{n 32}=}= & f_{d_{n 22}}
\end{aligned}
$$

Auxiliary barrier factors for the waste form barrier factor are defined by 
Equations $2.67,2.68,2.69,2.70,2.71,2.72$ and 2.73 [8].

$$
\begin{aligned}
& f_{w_{n 11}}=\quad 0.1,0.01,0.001 \quad \text { with prevention of } \\
& \text { dispersion } \\
& 1.0 \quad \text { without prevention of } \\
& \text { dispersion } \\
& f_{w_{n 12}}=\quad 0.1,0.01 \quad, \quad \text { with transport inhibition } \\
& 1.0 \text { without transport inhibition (2.68) } \\
& f_{w_{n 21}}=\quad f_{w_{n 12}} \\
& f_{w_{n 22}}=\quad 1.15 \cdot 10^{+0} \quad \text { for isotopes of } H \\
& 5.76 \cdot 10^{-3} \text {. for isotopes of } C \\
& 1.48 \cdot 10^{-2} \quad \text { for isotopes of } \mathrm{Fe}, \mathrm{Co}, \mathrm{Ni} \\
& 9.86 \cdot 10^{-3} \quad \text { for isotopes of } \mathrm{Sr} \\
& 1.11 \cdot 10^{-2} \quad \text { for isotopes of } \mathrm{Nb} \\
& 1.15 \cdot 10^{-1} \quad \text { for isotopes of } T c, I \\
& 1.62 \cdot 10^{-4} \quad \text { for isotopes of } C s \\
& 1.25 \cdot 10^{-4} \quad \text { for isotopes of } U \\
& 4.67 \cdot 10^{-4} \quad \text { for isotopes of } \mathrm{Np}, \mathrm{Pu}, \mathrm{Cm} \\
& 4.11 \cdot 10^{-3} \quad \text { for isotopes of } \mathrm{Am} \\
& 3.42 \cdot 10^{-3} \quad \text { for all other radionuclides } \\
& f_{w_{n 23}}=\quad 1.0 \quad \text { without prevention of dispersion } \\
& 1.0,0.25,0.0625 \quad \text { without waste segmentation } \\
& 1.0,0.25,0.0625,0.015625 \text { with waste segmentation } \\
& f_{w_{n 24}}=\quad 1.0 \quad \text { contact time fraction } \\
& f_{w_{n 31}}=\quad 0.8 \quad \text { with low metal content }
\end{aligned}
$$


Auxiliary barrier factors for the site selection barrier factor are defined by Equations 2.74, 2.75 and 2.76 [8].

$$
\begin{aligned}
& f_{s_{n 11}}=3.18 \cdot 10^{-11} \\
& f_{s_{n 21}}=0.5 \\
& f_{s_{n 31}}=0.27
\end{aligned}
$$

The auxiliary barrier factor $f_{w_{n 24}}$ of the waste form barrier factor determines how long radioactive waste has been in contact with (ground)water. The auxiliary barrier factor $f_{w_{n 23}}$ of the waste form barrier factor determines to which extent radioactive waste will be segmented according to its chemical properties.

The auxiliary barrier factors used in this study are given in Table 2.6.

\begin{tabular}{|ll|}
\hline \hline Factor & Value \\
\hline$f_{o_{n 11}}$ & $=1.0$ \\
$f_{d_{n 11}}$ & $=0.75$ \\
$f_{d_{n 12}}$ & $=0.1$ \\
$f_{w_{n 11}}$ & $=0.1$ \\
$f_{w_{n 12}}$ & $=0.1$ \\
$f_{w_{n 23}}$ & $=1.0$ \\
$f_{w_{n 31}}$ & $=0.1$ \\
\hline
\end{tabular}

Table 2.6: Auxiliary Barrier Factors used in this Study, Agriculture Scenario 


\section{Chapter 3}

\section{Methods of the Study}

\subsection{Introduction}

The methodology employed to obtain the radioactive waste parameters as defined in Chapter 2 is based on the irradiation of homogenized reactor components in the neutron flux of a nuclear reactor and the subsequent discharge of the components from the reactor. It is assumed that the neutron flux does not change in time, although a changing nuclide composition of the components could lead to minute temporal variations. Neutron flux and nuclide composition of a component have the strongest influence on the activation of this component.

Components are defined by their function within a reactor, as detailed in Section 4.1. Components of fast fission reactors are located in the driver, the internal breeder and the radial breeder section and in the reflector and shield section of the reactor. Components of fusion reactors are located in the inboard and the outboard section of the reactor.

Radioactive and non-radioactive nuclides in those components will build up and decay during irradiation and after discharge. Build-up and decay are simulated by computer codes. ORIGEN-II plays the central role for fast fission reactors and $R E A C-I I$ plays the central role for fusion reactors. Radioactive waste parameters of radioactive nuclides and of components are determined at $100 s, 1 h, 1 d, 1 w, 1 y, 10 y, 100 y, 1,000 y$ and 10,000y after 
discharge. However, only radioactive waste parameters of components are used to conduct the comparison.

Homogenization of components substantially lessens the need for computer memory and disk storage space and for computer $C P U$-time, because not every material of a component has to be accounted for separately, especially, if those materials consist of similar elements. Also the efficiency in running the computer codes is increased. However, homogenization has a strong, although not distorting, effect on the accuracy. All components will be affected by homogenization in a similar way, so that a comparison of components, i.e. radioactive waste, still leads to valid conclusions.

Nuclide data libraries that have to be provided for neutron transportation and neutron activation computer codes also have a strong effect on the accuracy. Section 1.2 shows that radioactive waste from fast fission and fusion reactors contains different nuclides, so that nuclide data libraries for fast fission calculations usually do not feature the complete set of nuclides necessary for fusion calculations, and vice versa. Although some overlapping can be found, this does not hold true for actinides and a major part of fission products. However, the computer codes employed in this study use combined nuclide data libraries for fast fission and fusion calculations.

The methods used to determine radioactive waste parameters of radioactive waste from fast fission and fusion reactors are slightly different, so the respective methods will be outlined separately. Both methods can best be illustrated by describing the integration of the different computer codes employed. However, it is useful to portray the computer codes separately prior to the description of their integration. Plot codes shall not be portrayed.

\subsection{Fast Fission Reactors}

\subsubsection{ORIGEN-II Neutron Activation Code}

$O R I G E N-I I$ solves for a homogenized reactor component and for different 
times the coupled linear equation system describing the generation and destruction of nuclides by neutron absorption and radioactive decay. A single neutron energy group and $18 \mathrm{\gamma} /$ photon energy groups are used by ORIGEN-II; the latter ones are ignored in this study, because photons do not contribute to activation. ORIGEN-II is run separately for each component of the reactor. Detailed information about ORIGEN-II is provided in Reference [7].

An ASCII nuclide neutron cross section library and an ASCII nuclide decay data library come along with the code package. However, those nuclide data libraries only contain data for thermal reactors and the available version of ORIGEN-II was a thermal version also. Therefore, nuclide data libraries with data for fast reactors as provided by Reference [14] were used. Also, the thermal version of ORIGEN-II was transformed into a fast version.

The input file to ORIGEN-II must specify the composition of the component that is to be considered, the times for which the linear equation system is to be solved and the neutron flux effective at those times (including a vanishing neutron flux for times after discharge). The composition must be given as the masses of the nuclides contained in the component (in [g]). This already reflects the homogenization. Absolute radioactivities of the nuclides of the component at above times are calculated subsequently. Absolute radioactivities of the nuclides and the ones of the component at above times are written to an ASCII output file.

\subsection{Fusion Reactors}

\subsubsection{VMIBOB Code}

$V M I B O B$ determines the volumes and masses of reactor components and component materials and the volume fractions of materials in components. It therefore is the basis of homogenization of the components. However, to avoid too strong a homogenization, especially large components have to 
be divided into subcomponents, which then are treated as separate components. $Y M I B O B$ is run once for all components of a reactor.

In order to determine volumes, a certain geometry has to be assumed. Principally there are two ways of geometrically modelling a reactor without taking the extremely complicated non-perfect toroidal form into account. The first alternative cuts the torus apart perpendicular to its major radius circumference; the second alternative cuts the torus apart along its major radius circumference. Both types of cuts are done parallel to the torus rotational axis.

The first alternative yields two circles of minor radius as the cut lines and subsequent to the cut, the torus is bent into a straight cylinder, thereby increasing the original major radius to infinity. Hence the major radial circumference of the torus becomes the cylinder axis. This alternative is referred to as One-Cylinder approximation. Each component then is approximated as a cylindrical layer with inner and outer radii greater than the minor radius.

The second alternative yields four circles of major radius as the cut lines and subsequent to the cut, the two resulting parts of the torus are bent into two cylinders - inboard and outboard cylinder - by increasing the original minor radius to infinity. The inboard cylinder radius is equal to the major radius minus the minor radius, the outboard cylinder radius is equal to the major radius plus the minor radius. Hence the rotational axis of the torus becomes the cylinder axis. This alternative is referred to as Two-Cylinder approximation. Now, the reactor has inboard and outboard sections. Each inboard component is approximated as a cylindrical layer with inner and outer radii less than the inboard cylinder radius, each outboard component is approximated as a cylindrical layer with inner and outer radii greater than the outboard cylinder radius.

An elongation $\kappa \geq 2$ results in a pronounced $D$ shape of the non-perfect torus cross section. This renders the Two-Cylinder approximation much 
more appropriate than the One-Cylinder approximation.

Inner and outer component radii $R_{c_{1}}$ and $R_{c_{o}}$ of the single components are given by the radial build of the components at the reactor midplane, which is the plane cutting the non-perfect torus in half at its equator. The height $H_{c}$ of these components must be estimated, e.g. as two times the minor radius times the elongation plus the sum of all thicknesses $R_{c_{o}}-R_{c_{1}}$ of all components. The volume $V_{c}$ of a component can then be calculated according to Equation 3.1. Under this scheme, components that are not located at the reactor midplane can not be accounted for reasonably well.

$$
V_{c}=\pi H_{c}\left(R_{c_{o}}^{2}-R_{c_{1}}^{2}\right)
$$

The calculation of the volume fraction of a material in a component is facilitated, if each material $m$ is approximated as a cylindrical layer of inner radius $R_{m c_{1}}$ and outer radius $R_{m c_{o}}$ within a component $c$, where the radii are given by the radial build of the materials at the reactor midplane. The volume $V_{m c}$ of a material in a component then is given by Equation 3.2 and the volume fraction is calculated according to Equation 3.3.

$$
\begin{gathered}
V_{m c}=\pi H_{c}\left(R_{m c_{o}}^{2}-R_{m c_{\imath}}^{2}\right) \\
\eta_{m c}=\frac{V_{m c}}{V_{c}}=\frac{R_{m c_{o}}^{2}-R_{m c_{1}}^{2}}{R_{c_{o}}^{2}-R_{c_{1}}^{2}}
\end{gathered}
$$

The mass $m_{m c}$ of a material in a component, the mass $m_{c}$ and the mass density $\rho_{c}$ of a component then can be calculated by Equations 3.4, 3.5 and 3.6 , where $\rho_{m}$ is the mass density of the material.

$$
\begin{gathered}
m_{m c}=\rho_{m} V_{m c} \\
m_{c}=\sum_{m=1}^{n_{m}} m_{m c}=\sum_{m=1}^{n_{m}} \rho_{m} V_{m c}
\end{gathered}
$$




$$
\rho_{c}=\frac{\sum_{m=1}^{n_{m}} m_{m c}}{V_{c}^{r}}=\frac{\sum_{m=1}^{n_{m}} \rho_{m} V_{m c}}{V_{c}^{r}}=\sum_{m=1}^{n_{m}} \eta_{m c} \rho_{m}
$$

The input file to $V M I B O B$ must specify minor and major radius of the reactor, the thickness $R_{m c_{o}}-R_{m c_{i}}$ and the weight density $\rho_{m}$ of the materials in the components. The radii $R_{c_{i}}, R_{c_{o}}, R_{m c_{i}}$ and $R_{m c_{o}}$, the volumes and the masses of the materials and the components and the volume fractions of the materials in the components are then calculated and written to an ASCII output file.

The actual material and component shape is too complicated to be considered exactly. But it appears reasonable to make the above approximations, because retracting and protruding parts of an actual material or component can throughout average themselves out and thus the actual volume can be close to the approximated volume. The principle of averaging out might hold true also for the assumption of pure cylindrical geometry, although the more exact treatment would require to consider the reactor as a non-perfect torus with $D$ shaped cross section.

\subsubsection{ONEDANT Neutron Transportation Code}

One-Dimensional Diffusion-Accelerated Neutral-Particle Transport (ONE$D A N T$ ) solves for a reactor the one-dimensional multienergy group neutron transport equation. With the Two-Cylinder approximation as described in Section 3.3.1, the one dimension is given by the cylinder major radial direction. 30 neutron energy groups and $12 \gamma /$ photon energy groups are used by $O N E D A N T$; the latter ones are ignored in this study because photons do not contribute to activation. Spatial discretization of the one dimension by means of a fine and coarse grid is necessary. ONEDANT is run once for all homogenized reactor components. Detailed information about ONEDANT is provided in Reference [32]. 
An $A S C I I$ nuclide neutron cross section library comes along with the code package. The input file to $O N E D A N T$ must specify the radial build of the components at the reactor midplane, the volume fraction of each material in a component (calculated by VMIBOB) and the concentration of each element in a material (in $\left[\frac{1}{b a r n \cdot c m}\right]$ ). Also, the radial build of the components at the reactor midplane must be split up into fine and coarse grid points to allow discretization.

The available nuclide neutron cross section library allows components to be built out of materials containing the following elements or isotopes thereof: $\mathrm{H}, \mathrm{He},{ }_{3}^{6} \mathrm{Li},{ }_{3}^{7} \mathrm{Li}, \mathrm{Be},{ }_{5}^{10} \mathrm{~B},{ }_{5}^{11} \mathrm{~B}, \mathrm{C}, \mathrm{O}, \mathrm{F}, \mathrm{Mg}, \mathrm{Al}, \mathrm{Si}, \mathrm{P}, \mathrm{S}, \mathrm{Ti}, \mathrm{V}$, $\mathrm{Cr}, \mathrm{Mn}, \mathrm{Fe}, \mathrm{Ni}, \mathrm{Mo}, \mathrm{Cu}, \mathrm{W}, \mathrm{Pb}$.

$O N E D A N T$ assumes the entire major radial $D$-T-plasma extension as a source of neutrons with energy $E_{N}=14.08 \mathrm{MeV}$. ONEDANT performs calculations for a $H=1 \mathrm{~cm}$ high slice of the Two-Cylinder approximation of the reactor, cut out at the reactor midplane. A normalized neutron flux for each fine grid point then is calculated and written to a Binary output file. Normalization means that one single neutron per time is assumed to enter the cylindrical first wall of the $1 \mathrm{~cm}$ high slice. In order to obtain the denormalized neutron flux, ONEDANT's normalized neutron flux has to be multiplied by a denormalization factor, i.e. the actual number of neutrons per time entering the cylindrical first wall of the $1 \mathrm{~cm}$ high slice.

This can be derived from the neutron first wall load by knowing the area of the $1 \mathrm{~cm}$ high cylindrical first wall. The product of neutron first wall load and this first wall area, divided by $E_{N}$ then gives the actual number of neutrons per time entering the first wall. If $R_{F W_{1 B}}$ and $R_{F W_{O B}}$ denote the radii of the inboard and outboard first wall and $L_{F W_{N}}$ is the neutron first wall load, then the denormalization factor $\Phi_{0}$ is given by Equation 3.7.

$$
\Phi_{0}=2 \cdot \pi \cdot H \cdot\left(R_{F W_{I B}}+R_{F W_{O B}}\right) \cdot \frac{L_{F W_{N}}}{E_{N}}
$$




\subsubsection{FLXWRT Code}

FLXWRT constitutes the interface code between ONEDANT and REACII. The normalized neutron flux of the reactor calculated by ONEDANT is needed only at particular radii of the homogenized reactor components. Those radii in terms of $O N E D A N T$ fine grid points are required to run FLXWRT. Furthermore, the ONEDANT denormalization factor $\Phi_{0}$ must be specified. FLXWRT is run once for all components of a reactor.

FLXWRT then reads the normalized 30 energy group neutron flux at the particular radii of the components from ONEDANT's Binary output file and multiplies it by the denormalization factor. The code then widens the denormalized 30 energy group neutron flux into a denormalized 52 energy group neutron flux. This neutron flux at the particular radii of the components then is written to a Binary and an $A S C I I$ output file. The Binary output file is directly suitable as a neutron flux library for $R E A C-I I$, while the $A S C I I$ output file is directly suitable as a neutron flux library for AVEFLUX.

\subsubsection{AVEFLUX Code}

$A V E F L U X$ determines the volume average neutron flux and the radius of this neutron flux within the homogenized reactor components. A component is approximated as a cylindrical layer of corresponding inner radius, outer radius and height. AVEFLUX is based on the assumption, that the neutron flux shape within a component is essentially an exponential one, which is an appropriate assumption for homogenized components. The 52 energy group neutron flux is collapsed into a thermal, an epithermal and a fast energy group neutron flux. The radii of the volume average neutron flux for those three collapsed energy groups turn out to be almost identical. AVEFLUX is run once for all components of a reactor.

The user must supply an $A S C I I$ neutron flux library (calculated by FLXWRT) with the neutron flux $\Phi_{c_{i}}$ and $\Phi_{c_{o}}$ given at the inner and outer 
radii of the components. The input file to AVEFLUX must specify those inner and outer radii $R_{c_{i}}$ and $R_{c_{o}}$. The volume average neutron flux of the components and the radius of this neutron flux then are written to an ASCII output file.

In cylindrical coordinates the volume average neutron flux $\Phi_{c_{a}}$ in a component $c$ can be derived as given in Equation 3.11. $\mu_{c}$ is the spatial neutron flux decay constant and $\Phi_{c}(r)$ the neutron flux shape within component $c$, and $H_{c}$ is its height.

$$
\begin{aligned}
\Phi_{c_{a}} & =\frac{\int_{V} \Phi_{c}(r) d V}{\int_{V} d V} \\
\Phi_{c}(r) & =\Phi_{c_{i}} e^{-\mu_{c}\left(r-R_{c_{i}}\right)} \\
d V & =2 \pi H r d r \\
\Phi_{c_{a}} & =\frac{\Phi_{c_{i}} e^{\mu_{c} R_{c_{i}} \int_{R_{c_{i}}}^{R_{c_{o}}} r e^{-\mu_{c} r} d r}}{R_{c_{o}}^{2}-R_{c_{i}}^{2}}
\end{aligned}
$$

After performing the integration over the exponential neutron flux shape within the given boundaries, the volume average neutron flux results as Equation 3.12.

$$
\Phi_{c_{a}}=\frac{2 \Phi_{c_{i}}}{\mu_{c}^{2}} \frac{\left(-\mu_{c} R_{c_{o}}-1\right) e^{-\mu_{c}\left(R_{c_{o}}-R_{c_{i}}\right)}-\left(-\mu_{c} R_{c_{i}}-1\right)}{R_{c_{o}}^{2}-R_{c_{i}}^{2}}
$$

Inserting this volume average neutron flux into the general neutron flux shape, Equation 3.9, gives the radius $R_{c_{a}}$ at which the volume average neutron flux will be located within the component, Equation 3.13.

$$
R_{c_{a}}=R_{c_{i}}+\frac{\ln \Phi_{c_{a}}-\ln \Phi_{c_{i}}}{-\mu_{c}}
$$


The neutron flux decay constant $\mu_{c}$ can be derived from the radii $R_{c_{i}}$ and $R_{c_{o}}$ and the neutron flux at those radii by solving a linear system of two equations resulting from Equation 3.9.

$$
\begin{gathered}
\Phi_{c_{i}}=\Phi_{c_{i}} e^{-\mu_{c}\left(R_{c_{i}}-R_{c_{i}}\right)} \\
\Phi_{c_{o}}=\Phi_{c_{i}} e^{-\mu_{c}\left(R_{c_{o}}-R_{c_{i}}\right)} \\
-\mu_{c}=\frac{\ln \Phi_{c_{o}}-\ln \Phi_{c_{i}}}{R_{c_{o}}-R_{c_{i}}}
\end{gathered}
$$

For inboard components the neutron flux will increase with increasing radius $r$ and hence $\mu_{c}$ will be negative, for outboard components the neutron flux will decrease with increasing radius $r$ and hence $\mu_{c}$ will be positive.

Depending on how well the actual neutron flux shape resembles the exponential neutron flux shape, the radius of the volume average neutron flux will be more or less different from the radius at which the actual volume average neutron flux occurs. On the one hand, the volume average of an exponential neutron flux shape should give a more reasonable approximation of the actual radius of the volume average neutron flux than the one of a linear neutron flux shape would do.

On the other hand, the actual neutron flux shape might be significantly different from the exponential one, because homogenization smoothes out the peaks and troughs in an actual neutron flux shape due to particular materials in each component.

\subsubsection{COMCOMP Code}

COMCOMP determines the composition of the homogenized reactor components. A component comprises several materials, a material several elements and an element several isotopes. The composition is obtained as 
nuclide fractions of the single isotopes of the elements present in a component, i.e. as the number of nuclides of an isotope of an element per number of all isotopes of all elements of all materials of a component.

In the following derivation of the composition of a component in terms of nuclide fractions, the Avogadro number is denoted by the letter $A$, the symbols for number, concentration, mole number, mass, volume and atom or mole weight are $N, N^{*}, n, m, V$ and $M$, respectively. Mass densities are denoted by the letter $\rho$, nuclide, mass and volume fractions by the letters $a, \xi$ and $\eta$, respectively.

The fraction $a_{i e}$ of the number $N_{i e}$ of nuclides of isotope $i$ of element $e$ and the number $N_{e}$ of nuclides of all isotopes of element $e$ is usually given in the Chart of Nuclides as Equation 3.17.

$$
\begin{gathered}
a_{i e}=\frac{N_{i e}}{N_{e}} \\
N_{e}=\sum_{i=1}^{n_{i}(e)} N_{i e} \\
1=\sum_{i=1}^{n_{i}(e)} a_{i e}
\end{gathered}
$$

The number $N_{e m}$ of nuclides of all isotopes of element $e$ of material $m$ can be derived as Equation 3.21.

$$
\begin{aligned}
n_{e m} & =\frac{m_{e m}}{M_{e}}=\frac{\rho_{e}}{M_{e}} V_{e m} \\
N_{e m} & =n_{e m} A=\frac{\rho_{e} A}{M_{e}} V_{e m} \\
N_{e}^{*} & =\frac{N_{e m}}{V_{e m}}=\frac{\rho_{e} A}{M_{e}}
\end{aligned}
$$


The fraction $a_{e m}$ of the number $N_{e m}$ of nuclides of all isotopes of element $e$ of material $m$ and the number $N_{m c}$ of nuclides of all isotopes of all elements of material $m$ of component $c$ is written as Equation 3.23.

$$
a_{e m}=\frac{N_{e m}}{N_{m c}}
$$

The number $N_{m c}$ of nuclides of all isotopes of all elements of material $m$ of component $c$ can be derived as Equation 3.25.

$$
\begin{aligned}
& n_{m c}=\frac{m_{m c}}{M_{m}}=\frac{\rho_{m}}{M_{m}} V_{m c} \\
& N_{m c}=n_{m c} A=\frac{\rho_{m} A}{M_{m}} V_{m c} \\
& N_{m}^{*}=\frac{N_{m c}}{V_{m c}}=\frac{\rho_{m} A}{M_{m}}
\end{aligned}
$$

This procedure requires proper definition of $\rho_{m}$ and $M_{m}$ according to Equations 3.27 and 3.28 .

$$
\begin{aligned}
\rho_{m} & =\frac{m_{m c}}{V_{m c}}=\frac{m_{m c}}{\sum_{e=1}^{n_{e}(m)} V_{e m}}=\frac{m_{m c}}{\sum_{e=1}^{n_{e}(m)} \frac{m_{e m}}{\rho_{e}}}=\frac{1}{\sum_{e=1}^{n_{e}(m)} \frac{m_{e m}}{m_{m c}} \frac{1}{\rho_{e}}} \\
& =\frac{1}{\sum_{e=1}^{n_{e}(m)} \frac{\xi_{e m}}{\rho_{e}}} \\
M_{m} & =\frac{m_{m c}}{n_{m c}}=\frac{m_{m c}}{\sum_{e=1}^{n_{e}(m)} n_{e m}}=\frac{m_{m c}}{\sum_{e=1}^{n_{e}(m)} \frac{m_{e m}}{M_{e}}}=\frac{1}{\sum_{e=1}^{n_{e}(m)} \frac{m_{e m}}{m_{m c}} \frac{1}{M_{e}}} \\
& =\frac{1}{\sum_{e=1}^{n_{e}(m)} \frac{\xi_{e m}}{M_{e}}}
\end{aligned}
$$

Substituting $\rho_{m}$ and $M_{m}$ into Equation 3.25 shows that these are the right definitions since together with Equation 3.21 it yields Equations 3.29 and 3.30 . 


$$
\begin{aligned}
N_{m c} & =\sum_{e=1}^{n_{e}(m)} N_{e m} \\
1 & =\sum_{e=1}^{n_{e}(m)} a_{e m}
\end{aligned}
$$

The usage of $n_{m c}=\sum_{e=1}^{n_{e}(m)} n_{e m}$ in Equation 3.28 is allowed because of Equations 3.21, 3.25 and 3.29. $a_{\mathrm{em}}$ can then be given as Equation 3.31.

$$
a_{e m}=\frac{N_{e m}}{N_{m c}}=\frac{n_{e m}}{n_{m c}}=\frac{m_{e m}}{M_{e}} \frac{M_{m}}{m_{m c}}=\xi_{e m} \frac{M_{m}}{M_{e}}
$$

The fraction $a_{m c}$ of the number $N_{m c}$ of nuclides of all isotopes of all elements of material $m$ of component $c$ and the number $N_{c}$ of nuclides of all isotopes of all elements of all materials of component $c$ is written as Equation 3.32.

$$
a_{m c}=\frac{N_{m c}}{N_{c}}
$$

The number $N_{c}$ of nuclides of all isotopes of all elements of all materials of component $c$ can be derived as Equation 3.34.

$$
\begin{aligned}
n_{c} & =\frac{m_{c}}{M_{c}}=\frac{\rho_{c}}{M_{c}} V_{c} \\
N_{c} & =n_{c} A=\frac{\rho_{c} A}{M_{c}} V_{c} \\
N_{c}^{*} & =\frac{N_{c}}{V_{c}}=\frac{\rho_{c} A}{M_{c}}
\end{aligned}
$$

This procedure requires proper definition of $\rho_{c}$ and $M_{c}$ according to Equations 3.36 and 3.37 . 


$$
\begin{aligned}
\rho_{c} & =\frac{m_{c}}{V_{c}}=\frac{\sum_{m=1}^{n_{m}(c)} m_{m c}}{V_{c}}=\frac{\sum_{m=1}^{n_{m}(c)} V_{m c} \rho_{m}}{V_{c}}=\sum_{m=1}^{n_{m}(c)} \frac{V_{m c}}{V_{c}} \rho_{m} \\
& =\sum_{m=1}^{n_{m}(c)} \eta_{m c} \rho_{m} \\
M_{c} & =\frac{m_{c}}{n_{c}}=\frac{m_{c}}{\sum_{m=1}^{n_{m}(c)} n_{m c}}=\frac{m_{c}}{\sum_{m=1}^{n_{m}(c)} \frac{m_{m c}}{M_{m}}}=\frac{1}{\sum_{m=1}^{n_{m}(c)} \frac{m_{m c}}{m_{c}} \frac{1}{M_{m}}} \\
& =\frac{1}{\sum_{m=1}^{n_{m}(c)} \frac{\rho_{m} V_{m c}}{\rho_{c} V_{c}} \frac{1}{M_{m}}}=\frac{1}{\sum_{m=1}^{n_{m}(c)} \eta_{m c} \frac{\rho_{m}}{\rho_{c}} \frac{1}{M_{m}}}
\end{aligned}
$$

Substituting $\rho_{c}$ and $M_{c}$ into Equation 3.34 shows that these are the right definitions since together with Equation 3.25 it yields Equations 3.38 and 3.39 .

$$
\begin{aligned}
N_{c} & =\sum_{m=1}^{n_{m}(c)} N_{m c} \\
1 & =\sum_{m=1}^{n_{m}(c)} a_{m c}
\end{aligned}
$$

The usage of $n_{c}=\sum_{m=1}^{n_{m}(c)} n_{m c}$ in Equation 3.37 is allowed because of Equations 3.25, 3.34 and 3.38. $a_{m c}$ can then be given as Equation 3.40.

$$
a_{m c}=\frac{N_{m c}}{N_{c}}=\frac{n_{m c}}{n_{c}}=\frac{m_{m c}}{M_{m}} \frac{M_{c}}{m_{c}}=\frac{\rho_{m} V_{m c}}{M_{m}} \frac{M_{c}}{\rho_{c} V_{c}}=\eta_{m c} \frac{\rho_{m}}{\rho_{c}} \frac{M_{c}}{M_{m}}
$$

The fraction $a_{i c}$ of the number $N_{i e}$ of nuclides of isotope $i$ of element $e$ and the the number $N_{c}$ of nuclides of all isotopes of all elements of all materials of component $c$ is written as: 


$$
a_{i c}=\frac{N_{i e}}{N_{c}}
$$

This can be detailed as in Equations $3.42,3.43$ and 3.44.

$$
\begin{aligned}
a_{e m} a_{m c} & =\frac{N_{e m}}{N_{m c}} \frac{N_{m c}}{N_{c}}=\frac{N_{e m}}{N_{c}} \\
a_{e c} & =\frac{N_{e}}{N_{c}}=\sum_{m=1}^{n_{m}(c)} \frac{N_{e m}}{N_{c}}=\sum_{m=1}^{n_{m}(c)} a_{e m} a_{m c} \\
a_{i c} & =\frac{N_{i e}}{N_{c}}=\frac{N_{i e}}{N_{e}} \frac{N_{e}}{N_{c}}=a_{i e} \sum_{m=1}^{n_{m}(c)} a_{e m} a_{m c}
\end{aligned}
$$

Eventually substituting the known for the unknown variables, i.e. Equation 3.31 for $a_{e m}$ and Equation 3.40 for $a_{m c}$, and using Equations 3.28, 3.36 and 3.37 results in the formula as given in Equation 3.45.

$$
a_{i c}=a_{i e} \sum_{m=1}^{n_{m}(c)} \frac{\xi_{e m} \eta_{m c} \rho_{m}}{M_{e} \sum_{m=1}^{n_{m}(c)}\left(\eta_{m c} \rho_{m} \sum_{e=1}^{n_{e}(m)} \frac{\xi_{e m}}{M_{e}}\right)}
$$

If $a_{e m}$ is given instead of $\xi_{e m}$, then the calculation of $\xi_{e m}$ from $a_{e m}$ is necessary, because the calculation of $M_{m}$ requires $\xi_{\mathrm{em}}$. However, Equation 3.45 can be recast to yield Equation 3.46, using Equations 3.28 and 3.31.

$$
a_{i c}=a_{i e} \sum_{m=1}^{n_{m}(c)} \frac{a_{e m} \eta_{m c} \rho_{m}}{\sum_{e=1}^{n_{e}(m)} \frac{\xi_{e m}}{M_{e}} \sum_{m=1}^{n_{m}(c)}\left(\eta_{m c} \rho_{m} \sum_{e=1}^{n_{e}(m)} \frac{\xi_{e m}}{M_{e}}\right)}
$$

Thus, the calculation requires the nuclide fraction $a_{i e}$ of isotopes $i$ of elements $e$, the atom or mole weight $M_{e}$ of elements $e$, the weight fraction $\xi_{e m}$ or nuclide fraction $a_{e m}$ of elements $e$ of materials $m$, the weight density 
$\rho_{m}$ of materials $m$ and the volume fraction $\eta_{m c}$ of materials $m$ of component $c$ (calculated by $V M I B O B$ ); all those composition specifications are provided as FORTRAN-DATA statements for the components and hence COMCOMP is run once for all components of a reactor. The composition of the components is written to an $A S C I I$ output file.

\subsubsection{REAC-II Neutron Activation Code}

The program system REAC-II consistes of the three codes SREAC, SLST$C O M$ and $S L I B . S L I B$ is a $R E A C-I I$ auxiliary code that converts an $A S C I I$ composition library (calculated by COMCOMP) to a Binary composition library. $S L I B$ is run once for all homogenized reactor components.

$S R E A C$ solves for a component and for different times the coupled linear equation system describing the generation and destruction of nuclides by neutron absorption and radioactive decay. 52 neutron energy groups and $11 \mathrm{\gamma} /$ photon energy groups are used by $S R E A C$; the latter ones are ignored in this study, because photons do not contribute to activation. SREAC is run separately for each component of a reactor. Detailed information about $R E A C$-II is provided in Reference [30].

A Binary nuclide neutron cross section library and a Binary nuclide decay data library come along with the code package. The user must supply a Binary composition library (calculated by $S L I B$ ), where the composition of the components is given as nuclide fractions, and a Binary neutron flux library (calculated by FLXWRT), where the neutron flux must be given at the radius of the volume average neutron flux of a component (calculated by $A V E F L U X)$. The input file to $S R E A C$ must specify the component for which composition and neutron flux in those libraries are requested, the times for which the linear equation system is to be solved and the fraction of the neutron flux effective at those times (including a vanishing neutron flux at times after discharge). The concentrations of the nuclides of a component 
at the above times then are calculated and written to a Binary output file.

Using this Binary output file and the Binary nuclide decay data library, SLSTCOM then calculates specific radioactivities and specific $\gamma$-dose rates of the nuclides of a component by simply multiplying the concentrations of nuclides by corresponding decay constants and $\boldsymbol{\gamma}$-dose rate conversion factors, respectively. Specific radioactivities and specific $\gamma$-dose rates of the nuclides and the ones of the component at the above times are written to an ASCII output file. SLSTCOM is run once for each component of a reactor.

REAC-II had not yet implemented all of its potentials as of the time of this study. Among the missing capabilities are the calculation of the decay power and the Biological Hazard Potential from data like the decay heat per decay, the Maximum Permissable Concentration and the specific radioactivities of the nuclides in the component.

\subsection{OPCPOST \& RECPOST Codes}

$O P C P O S T$ and RECPOST determine for all homogenized reactor components at different times the specific and absolute radioactive waste parameters as listed in Chapter 2. The codes read the radioactivities calculated by $O R I G E N-I I$ and $R E A C-I I$ from the respective $A S C I I$ output files. However, ORIGEN-II gives absolute radioactivities and $R E A C-I I$ gives specific radioactivities. In addition, the formats of the aforesaid $A S C I I$ output files are different. Absolute radioactivities are converted to specific radioactivities upon completion of the reading procedure. Specific radioactivities of the radionuclides of components at the above times then form the basis of all further calculations by $O P C P O S T$ and $R E C P O S T$, which use the formulas developed in Chapter 2. OPCPOST and RECPOST are run once for all components of a reactor.

Although the above difference is the only difference between those codes, it appears reasonable to maintain two otherwise identical codes, because it 
allows maintenance of a clearly separated fast fission and fusion track on the computer.

$O P C P O S T$ and RECPOST allow to arbitrarily combine components to form new homogenized reactor components with corresponding radioactive waste parameters. In the case of OPCPOST, this is useful to calculate radioactive waste parameters of a component that represents the combination of corresponding components of the driver, internal breeder, radial breeder, reflector and shield sections. In the case of RECPOST, this is useful to calculate radioactive waste parameters of a component that represents the combination of corresponding components of the inboard and outboard sections, and also for subcomponents as defined in Section 3.3.1 to be recombined to components again.

$O P C P O S T$ and $R E C P O S T$ feature a great flexibility with respect to the nuclide data libraries used, since these are written in $A S C I I$ format and can be changed easily, as opposed to nuclide data libraries in Binary format. However, this places higher requirements on computer memory and disk storage space and $C P U$-time.

The user must supply an $A S C I I$ nuclide decay heat data library, an $A S C I I$ nuclide $M P C$ data library, an $A S C I I$ nuclide $W D R$ data library, an $A S C I I$ nuclide $P D C$ data library and one ORIGEN-II or REAC-II ASCII output file, respectively, for each component to be considered. The input files to OPCPOST and RECPOST must specify these ORIGEN-II or REAC-II ASCII output files, the volumes and solid volume fractions of the corresponding components, the components that are to be combined to form new components, the names of these components, the auxiliary barrier factors necessary to determine the intruder dose rates and several output control parameters.

Radioactivity, whole body $\boldsymbol{\gamma}$-dose rate, decay power and $B H P$ of all components at the above times on the one hand and $R W C$ indices and intruder dose rates of all components at the above times on the other hand 
are calculated and written to separate $A S C I I$ output files. In addition radioactive waste parameters of all nuclides of the components can be written to corresponding separate $A S C I I$ output files.

\subsection{Integration of the Codes}

\subsubsection{Fast Fission Reactors}

The nuclide neutron cross section data library and the nuclide decay data library as provided by Reference [14] have to be converted from an $A S C I I$ format suitable for the ORIGEN-II Mainframe version to an $A S C I I$ format suitable for the ORIGEN-II Personal Computer version. This conversion is done by the auxiliary code $D E C I B M$.

ORIGEN-II then calculates the absolute radioactivities of the nuclides contained in the homogenized reactor components at different times and makes them available to OPCPOST. OPCPOST then calculates the radioactive waste parameters of the components at the above times.

ORIGEN-II runs on a COMPAQ-DESKPRO-286 PC, as installed at the Department of Nuclear Engineering $(N E D)$ at the Massachusetts Institute of Technology, Cambridge, Massachusetts (MIT). DECIBM runs on a CRAY XM-P with two CPUs and $2 M$ egawords core memory, as installed at the National Energy Research Supercomputing Center (NERSC) of the Laurence Livermore National Laboratory ( $L L N L)$ at the University of California, Livermore, California (UCL). High core memory requirements necessitate that OPCPOST be run on a CRAYII with four CPUs and $128 \mathrm{Megawords}$ core memory, as installed at the NERSC of $L L N L$ at $U C L$.

Data transfer among all three computers is effected by the KERMIT and NETTY utilities. Only ORIGEN-II is commercially available. DECIBM was written by the author of this study. 


\subsubsection{Fusion Reactors}

$V M I B O B$ calculates for the reactor components the volume fractions of the materials contained therein. This is the basis of homogenization of the components. $V M I B O B$ makes the volume fractions available to $O N E D A N T$ and COMCOMP. ONEDANT then calculates the neutron flux in the reactor. FLXWRT makes available to AVEFLUX and REAC-II this neutron flux at the desired radii of the homogenized reactor components.

Under the computational scheme devised for this study, FLXWRT is run twice. The first run obtains the neutron flux at the inner and outer radii of the components. The code AVEFLUX then calculates the radii of the volume average neutron flux within the components. The second run of FLXWRT then obtains the neutron flux at these radii of the components. The corresponding neutron flux is assumed to be the representative neutron flux of each component.

COMCOMP homogenizes the components and makes available to $S L I B$ the corresponding composition. $S L I B$ then makes available this composition to the $R E A C-I I$ program system. SREAC calculates the concentrations of the nuclides contained in the components at different times. Subsequently SLSTCOM calculates the radioactivities and $\gamma$-dose rates of the nuclides and the components and makes them available to RECPOST. Auxiliary codes $D H T$ and $M P C$ generate $A S C I I$ nuclide decay heat data and $A S C I I$ nuclide $M P C$ data libraries used to run RECPOST. RECPOST eventually calculates all radioactive waste parameters of the components at the above times.

VMIBOB, COMCOMP, DHT and $M P C$ run on a DEC-VAX-11/780, as installed at the Plasma Fusion Center (PFC) at the Massachusetts Institute of Technology, Cambridge, Massachusetts (MIT). Both ONEDANT and REAC-II as well as FLXWRT and AVEFLUX run on a CRAYX-MP with two CPUs and 2Megawords core memory, as installed at the $\mathrm{Na}$ tional Energy Research Supercomputing Center (NERSC) of the Laurence Livermore National Laboratory (LLNL) at the University of Califormia, Liv- 
ermore, California (UCL). High core memory requirements necessitate that $R E C P O S T$ be run on a CRAY II with four CPUs and $128 \mathrm{M}$ egawords core memory, as installed at the NERSC of $L L N L$ at UCL. Only ONEDANT and $R E A C-I I$ are commercially available. FLXWRT already existed on the $C R A Y X-M P$ and all other codes were written by the author of this study. Data transfer among all three computers is effected by the NETTY utility. 


\section{Chapter 4}

\section{Description of the Reactors}

\subsection{Introduction}

Radioactive waste and radioactive waste parameters as defined in Chapter 2 are dependent on the nuclear reactors that are considered. The history and objectives of these reactors convey a feeling for their relative importance, the operation schedule and the design in combination with the methods as given in Chapter 3 allow the evaluation of radioactive waste and radioactive waste parameters.

The operation schedule provides the intensity and duration of irradiation of reactor components, the design provides the material that is irradiated in those components, which constitute the radioactive waste. The intensity of irradiation is expressed in terms of a neutron flux for fast fission reactors and in terms of a neutron first wall load for fusion reactors.

History and objectives, operation schedule and design of the reactors considered in this study shall therefore be described here. In addition, the description of the radioactive waste handling projected for the reactors enables the reader to appreciate important subtleties.

However, only the radioactive waste handling for fast fission reactors shall be outlined here, because no detailed information is available on radioactive waste handling for fusion reactors. Furthermore, only the chemical aspects of radioactive waste handling for fast fission reactors shall be 
concentrated on, because chemistry is the key issue.

\subsection{Fast Fission Reactors}

In this study, radioactive waste is identical to the solid fraction of the reactor components. The identification of those components is facilitated, if the reactor core is separated into a driver, internal breeder and radial breeder section.

A section contains a number of assemblies and an assembly consists of a duct and a number of fuel elements. A fuel element comprises fuel and clad. Fuel is composed of actindes, fission products and activation products.

In addition to the three core sections, there are a radial reflector section and a radial shield section. Assemblies of all sections feature a hexagonal cross section, caused by the triangular grid arrangement of the fuel elements in the driver and breeder sections.

Spacer wires wrapped around the clad of fuel elements shall not be considered in this study due to their minute volume. Coolant as well as thermal bond between fuel and clad of fuel elements are liquid at the time of discharge from the reactor and therefore are not considered, either. Also, the number of control and safety assemblies is small compared to the total number of assemblies, so that control and safety assemblies are ignored.

Thus, fuel clad and duct of the three core sections and reflector and shield of the reflector and shield sections shall be considered as components in this study. Clearly, these components are defined by distinct functions within a reactor. Note, that each component typically consists of only one material.

Radioactive waste handling with respect to fuel in both $E B R-I I$ and IFR mainly consists of reprocessing of discharged driver and breeder fuel in a self-sufficient fuel cycle. ${ }^{235} U$ and ${ }^{238} U$ are reprocessed chiefly from the driver, ${ }^{238} U$ and ${ }^{239} P u$ chiefly from the breeder section. The cycle is closed and can be characterized by breeding of fuel in the reactor and reprocessing 
of fuel in an adjacent fuel cycle facility. Ideally, only the make-up for the burned ${ }^{235} U$ and ${ }^{238} U$ enters the cycle and the major part of the fission and activation products leaves the cycle. All actinides remain in the cycle as fuel. Actinides in fuel of the three core sections therefore do not count as radioactive waste. Reactor and fuel cycle facility represent a fully integrated plant $[5,44]$.

All radioactive waste handling takes place behind massive shielding. This eliminates the need for extended cooling times before radioactive waste handling can take place. Consequently, reprocessing occurs only a few days after discharge in the case of $E B R-I I[44]$ and a few months after discharge in the case of IFR [5]. The inventory of radioactive waste in the self-sufficient closed fuel cycle can be kept low by rapid reprocessing $[5,44]$.

All three core sections use metallic fuel. In contrast to ceramic fuel, metallic fuel can be cast in pins and does not have to be sintered as pellets. Thereby, metallic fuel represents an important basis for the efficiency of the self-sufficient closed fuel cycle.

Furthermore, $E B R-I I$ and $I F R$ feature liquid sodium cooling and a pool type primary coolant loop under near-atmospheric pressure [44,46]. Typical neutron energies immediately after fission are at an average of $2 \mathrm{MeV}$, with a maximum of $10 \mathrm{MeV}$. Typical neutron energies in the reactor are at an

average of $100 \mathrm{keV}$. The typical neutron flux is at $10^{15} \frac{\mathrm{n}}{\mathrm{cm}^{2} \mathrm{~s}}$, with minimum and maximum values within a factor of 10 .

\subsubsection{The Experimental Breeder Reactor II}

\subsubsection{History \& Objectives}

During the latter part of World War II at the Metallurgical Laboratory of the University of Chicago, Enrico Fermi, Eugene Wigner, Glenn T. Seaborg and Walter H. Zinn initiated the fast fission breeder concept, triggered by a possible future need to extend the resources of fissionable material [44]. 
The efforts of particularly Walter $\mathrm{H}$. Zinn who became the first director of the Argonne National Laboratory $(A N L)$ - the new name of the old Metallurgical Laboratory - led to the Experimental Breeder Reactor I (EBR-I). The development of the $E B R-I$ driver and breeder fuel fabrication methods at $A N L$ took place as early as 1945. In 1948, the Austin Company was contracted to conduct the detailed design of EBR-I [44].

The experimental fast fission reactor was planned to be located at $A N L$ 's University of Chicago site, but the Atomic Energy Commission (AEC) had already determined a location in southeast Idaho to be the National Reactor Testing Station (NRTS) and in 1949 the prospective EBR-I site was moved there. The NRTS later became the Idaho National Engineering Laboratory (INEL). ANL is a contractor of NRTS/INEL and the ANL part of NRTS/INEL is often referred to as ANL's West site. The functions of the $A E C$ are now performed by the Nuclear Regulatory Commission (NRC, NUREG) [44].

The construction of EBR-I at $A N L$ 's West site began in October 1949, the reactor reached nuclear criticality in August 1951 and nominal thermal power on December 19th 1951. The reactor was shut down on December 30th 1963. EBR-I was taken from its stand-by status and declared a national historic landmark in 1966 and in 1974 it was opened to the public community [44].

The objectives of the EBR-I experiment were to demonstrate the breeding of fuel and to build up operating experience with fast fission reactors. These objectives were met by twelve successful years of $E B R-I$ operation with different cores. In particular, $E B R-I$ demonstrated a breeding ratio of approximately 1.27 with a ${ }^{239} \mathrm{Pu}$ driver fuel. The ${ }^{235} \mathrm{U}$ driver fuel had only been able to demonstrate conversion at a ratio of approximately unity [44].

However, a true extension of the resources of fissionable material requires reprocessing of fuel besides breeding of fuel. Reprocessing on an 
experimental and commercial scale requires a minimum fuel throughput for it to make sense. $E B R-I$ was not able to deliver this minimum throughput and consequently a second experimental fast fission reactor, the Experimental Breeder Reactor II (EBR-II) became necessary. The objective was to demonstrate fuel reprocessing by installing not only a reactor that would breed fuel, but also a Fuel Cycle Facility (FCF) that would reprocess fuel. The new plant was also sited at NRTS/INEL. It should be noted that the $E B R-I I$ project was not triggered by military reasons but by truly economic reasons [44].

Only two years after $E B R-I$ reached its nominal thermal power and after breeding was successfully demonstrated, $A N L$ embarked on the $E B R$ $I I / F C F$ project in December 1953 with a proposal to the $A E C$. Funding was authorized by the $A E C$ in July 1955 and the design of $E B R-I I$ and $F C F$ was initiated in 1957. The H. K. Ferguson Company was responsible for the $F C F$ part. The $E B R-I I$ fuel reprocessing was developed at $A N L$ in 1955. Construction of $E B R-I I / F C F$ was started in October 1957 and completed in December 1962. EBR-II reached nuclear criticality on November 11th 1963 and operation began in July 1964. FCF reprocessed the first $E B R-I I$ fuel as early as September 1964 and by April $1965 E B R-I I$ used the first reprocessed driver fuel [44].

The breeding-reprocessing interaction between $E B R-I I$ and $F C F$ continued until January 1969. At that time, $E B R-I I / F C F$ had successfully met its original objective and the mission of $E B R-I I / F C F$ was changed. The new mission was testing and examining irradiated fuel and structure as they might be used in future fast fission and fusion reactors. In the early years already, $E B R$-II had been identified as an useful high-temperature fast neutron irradiation facility, so that the change of mission came naturally [44].

This change of mission required different equipment in the FCF. Space 
limitations ultimately led to the removal of almost all the original $F C F$ equipment. Consequently in 1970 the FCF was renamed into Fuel Examination Facility $(F E F)$. In March 1975, a newly built plant, called the Hot Fuel Examination Facility (HFEF), started operation at $A N L$ 's west site, determined to primarily serve the Fast Flux Test Facility (FFTF), which at that time was under construction at the Hanford Engineering Development Laboratory (HEDL) site in Washinghton (FFTF started operation in 1981!). Later on, the $F E F$ and the $H F E F$ were renamed into $H F E F / S o u t h$ and $H F E F /$ North, respectively. The fuel for $E B R-I I$ is now fabricated in commercial and $A N L$ in-house facilities without reprocessing [44].

As early as 1957 it was understood that the radioactive waste produced by $E B R-I I / F C F$ would have to be transferred to the custody of the $A E C$ for storage at NRTS. A central radioactive waste storage facility serving all contractors of NRTS was in operation almost from NRTS's inception in 1949 on. The Idaho Chemical Processing Plant (ICPP) also had high level radioactive waste storage facilities. Due to the high activation of $E B R$ $I I / F C F^{\prime}$ s prospective radioactive waste, however, none of these facilities were suitable and a new one had to be erected. The site finally chosen is located approximately $800 \mathrm{~m}$ to the northeast of $E B R-I I / F C F$. The facility features monitored retrievable storage of high level radioactive waste, a design implemented with respect to future radioactive waste repositories yet to be found. The EBR-II/FCF storage facility was called Radioactive Scrap and Waste Facility $(R S W F)$. After submission of the proposal in January 1964 and its acceptance in May 1964, the $R S W F$ was ready for operation by the end of 1964 [44].

\subsubsection{Operation Schedule}

$E B R-I I$ operation phases may be defined by either the driver fuel with which experiments were run or the campaign under which experiments were 
run. The different driver fuels are discussed in Section 4.2.1.3, the different campaigns shall be briefly outlined here.

The first campaign ran from 1964 to 1969 , where breeding and reprocessing of fuel were demonstrated by $E B R-I I$ and $F C F$. This represents the operation phase concentrated on in this study, because breeding and reprocessing constituted the self-sufficient closed fuel cycle. However, this cycle was non-periodic due to its experimental character [44]. Accounting for reactor down times, the duration of this operation phase can be set to $4 y$ and is referred to as the reactor life time in subsequent sections of this study.

The second campaign ran from 1969 to 1977, where experiments were conducted under the Run-To-Cladding-Breach (RTCB) slogan. This meant operation of the reactor with a contaminated primary coolant loop. Actually, the first breached fuel element was encountered in May 1967 and the second fuel element breach occured by the end of 1967 , so that the RTCB campaign should rather begin in 1967. However, this would not allow the first campaign to be treated coherently and therefore 1969 has been chosen by the author of this study as the begin of the second campaign [44].

The third campaign now is running since 1977, where experiments are conducted under the Run-Beyond-Cladding-Breach $(R B C B)$ slogan. The first such experiment was conducted in January 1977 [44].

During the operation phase under consideration, defined by the first campaign, the thermal power output was restricted to a value of less than $62.5 M W_{t h}$. Starting power approach in July 1964, EBR-II reached $30 M W_{t h}$ in August 1964 and 37.5MWth in October 1964. By March $196545 M W_{t h}$ were reached and in August 1965 the power was raised to $50 M W_{t h}$. The nominal thermal power output of $62.5 M W_{t h}$ was installed in September 1969 , only after the original mission of $E B R-I I / F C F$ had been changed [44].

The change from "Mark-I" to "Mark-IA" fuel as defined in Section 4.2.1.3, 
the insteady thermal power output and the non-periodic self-sufficient closed fuel cycle during the operation phase from 1964 to 1969 as well as abundant but inconsistent information on EBR-II $[13,44,53,54]$ make it difficult to determine a simple operation schedule.

However, the choice of the "Mark-IA" fuel seems to be appropriate, because it was used over major parts of the above operation phase. A representative thermal power output of $50 \mathrm{MW}$ th and information provided in References [13] and [53] can be used to estimate the neutron flux in the three core sections and the shield section. Accordingly, $2.5 \cdot 10^{15} \frac{\mathrm{n}}{\mathrm{cm}^{2} \mathrm{~s}}$ for the driver and internal breeder section, $1.5 \cdot 10^{15} \frac{\mathrm{n}}{\mathrm{cm}^{2} \mathrm{~s}}$ for the radial breeder section and $1.0 \cdot 10^{15} \frac{\mathrm{n}}{\mathrm{cm}^{2} \mathrm{~s}}$ for the shield section appear to be reasonable.

Also, Reference [44] quotes a number of approximately 500 driver and internal breeder assemblies and 100 radial breeder assemblies that have been reprocessed in the $F C F$ to meet $E B R-I I / F C F$ 's original objective. Those assemblies have been irradiated for approximately $135 d$. In addition, the 162 shield assemblies cited in Section 4.2.1.3 were irradiated for the entire duration of the operation phase from 1964 to 1969. This information allows accounting for the non-periodic self-sufficient closed fuel cycle over the entire operation phase and is used subsequently to assess the radioactive waste generated by $E B R-I I$.

The rough approximation of the $E B R-I I$ operation schedule had to be made to allow for a consistent method as outlined in Chapter 3. The activation of $E B R-I I$ thus calculated is expected to be accurate only within an order of magnitude range.

\subsubsection{Design}

$E B R-I I$ has a nominal thermal power output of $62.5 \mathrm{MW}$. This is converted to a nominal electrical power output of $19.5 \mathrm{MW}$, yielding a thermal plant efficiency of $\eta_{t h}=0.31$. The sodium at the core inlet has an average 
temperature of $370^{\circ} \mathrm{C}$ and heats up to an average temperature of $505^{\circ} \mathrm{C}$ at the core outlet [44].

The driver section is composed of 127 assemblies. 14 of those 127 assemblies are fueled control and safety assemblies. Usually 8 of them are used for control purposes and the remaining 6 serve safety purposes. Above and below the driver section, the original $E B R-I I$ core featured an internal breeder section with $2 \times 113$ assemblies. The radial breeder section consisted of approximately 600 assemblies at that time. The shield section has 162 assemblies $[44,53]$.

The driver assemblies contain 91 fuel elements. Due to a smaller measure across the flats, the fueled control and safety assemblies contain only 61 fuel elements. The internal and radial breeder assemblies contain only 18 fuel elements because of a larger fuel element diameter [44,54]. The shield assemblies are assumed to be solid blocks.

Control and safety assemblies measure less across their flats, because they have to slide within ducts of the size of the rest of the assemblies. The extent to which the fueled part of the individually driven control and safety assemblies is pulled up into the driver section determines the reactor power. In order to maintain the neutron flux shape and reactivity, only few of the remaining 113 assemblies may be replaced by assemblies for irradiation experiments [44].

Today, the internal breeder section is replaced by a steel shield. Also, there are now steel reflectors in the two innermost rows of the radial breeder section. Both changes were made in order to maximize the neutron flux for irradiation experiments. In addition, it was found that the internal breeder section did not contribute significantly toward the overall breeding ratio $[44,54]$.

For this study, it is suggested that the radioactive waste produced by $E B R-I I$ is more realistically assessed, if the numbers of assemblies as given in Section 4.2.1.2 are used, rather than the numbers given here. Under this prerequisite, Tables 4.1 and 4.2 give volumes and masses of the clad, duct, 
fuel and shield components. Note that not all parameters were available in references, so some of the parameters represent estimates.

The $E B R-I I$ driver fuel contains approximately 5 weight - $\%$ of fissium, which serves as a fuel stabilizer and is defined in Section 4.4.3. The remainder of $95 w e i g h t-\%$ consists of 94.9 weight $-\%$ uranium and 0.1 weight $-\%$ plutonium with negligible traces of americium and curium. The uranium is chiefly ${ }^{238} U$ with ${ }^{235} U$ enriched to about 50 nuclide $-\%$. The plutonium is chiefly ${ }^{240} \mathrm{Pu}$ with ${ }^{239} \mathrm{Pu}$ enriched to 9.5 nuclide $-\%$. For the internal breeder and radial breeder fuel, natural or depleted uranium is chosen [44].

The clad and duct of all three core sections and the shield are made of SS304L. The approximate isotope and element composition of the ("MARK-IA") fuel component and the element composition of the clad, duct and shield components are given in Appendix A.

The original EBR-II driver fuel was enriched to 48.08 nuclide $-\%{ }^{235} \mathrm{U}$ and the corresponding fuel element was named "Mark-I". A later version "Mark-IA" had a slightly shorter fuel rod to allow for a greater fission gas volume and the associated criticality loss was compensated for by increasing the enrichment to 52.50 nuclide $-\%{ }^{235} U$. The maximum allowed burnup for "Mark-I" fuel elements with respect to the limited fission gas volume was set to 1.0 nuclide - \% and the improved version "Mark-IA", introduced in early 1966 , was able to stand $1.2 n u c l i d e-\%$. This limit was raised to 1.5 nuclide $-\%$ in early and $1.8 n u c l i d e-\%$ in late 1969. A final value of $2.6 n u c l i d e-\%$ was reached by 1975 [44].

After the change of mission of $E B R-I I / F C F$, yet another fuel element was devoloped, designated "Mark-II". "Mark-II" had a slightly thinner fuel rod than "Mark-IA" and the associated criticality loss was compensated for by increasing the enrichment again, this time to 67.00 nuclide $-\%{ }^{235} \mathrm{U}$. Also the clad length was increased, a modification that was avoided in changing from "Mark-I" to "Mark-IA" but became necessary for "Mark-II" in order to accommodate a higher fission gas volume due to a higher burnup. This 


\begin{tabular}{|lr|r|r|r|}
\hline \hline \multicolumn{2}{|c|}{ Parameter } & Driver & $\begin{array}{r}\text { Internal } \\
\text { Breeder }\end{array}$ & $\begin{array}{r}\text { Radial } \\
\text { Breeder }\end{array}$ \\
\hline$D_{c}$ & {$[\mathrm{~m}]$} & $4.5720 \cdot 10^{-3}$ & $9.1440 \cdot 10^{-3}$ & $9.1440 \cdot 10^{-3}$ \\
$\frac{P}{D_{c}}$ & {$[1]$} & 1.290 & 1.290 & 1.290 \\
$t_{c}$ & {$[\mathrm{~m}]$} & $2.2860 \cdot 10^{-4}$ & $3.0000 \cdot 10^{-4}$ & $3.0000 \cdot 10^{-4}$ \\
$S_{d}$ & {$[\mathrm{~m}]$} & $5.8166 \cdot 10^{-2}$ & $5.8166 \cdot 10^{-2}$ & $5.8166 \cdot 10^{-2}$ \\
$t_{d}$ & {$[\mathrm{~m}]$} & $1.0000 \cdot 10^{-3}$ & $1.0000 \cdot 10^{-3}$ & $1.0000 \cdot 10^{-3}$ \\
$D_{f}$ & {$[\mathrm{~m}]$} & $3.6576 \cdot 10^{-3}$ & $7.3152 \cdot 10^{-3}$ & $7.3152 \cdot 10^{-3}$ \\
$H_{c}$ & {$[\mathrm{~m}]$} & $4.6196 \cdot 10^{-1}$ & $1.1176 \cdot 10^{+0}$ & $1.4605 \cdot 10^{+0}$ \\
$H_{d}$ & {$[\mathrm{~m}]$} & $4.6196 \cdot 10^{-1}$ & $1.8705 \cdot 10^{+0}$ & $2.3324 \cdot 10^{+0}$ \\
$H_{f}$ & {$[\mathrm{~m}]$} & $3.4290 \cdot 10^{-1}$ & $1.1176 \cdot 10^{+0}$ & $1.4605 \cdot 10^{+0}$ \\
\hline$N_{a}$ & {$[1]$} & 500 & 500 & 100 \\
$n$ & {$[1]$} & 91 & 18 & 18 \\
\hline$V_{c}$ & {$\left[\mathrm{~m}^{3}\right]$} & $1.4410 \cdot 10^{-6}$ & $9.3155 \cdot 10^{-6}$ & $1.2174 \cdot 10^{-5}$ \\
$V_{d}$ & {$\left[\mathrm{~m}^{3}\right]$} & $9.3082 \cdot 10^{-5}$ & $3.7689 \cdot 10^{-4}$ & $4.6997 \cdot 10^{-4}$ \\
$V_{f}$ & {$\left[\mathrm{~m}^{3}\right]$} & $3.6029 \cdot 10^{-6}$ & $4.6971 \cdot 10^{-5}$ & $6.1382 \cdot 10^{-5}$ \\
$N_{\mathrm{a}} n V_{c}$ & {$\left[\mathrm{~m}^{3}\right]$} & $6.5566 \cdot 10^{-2}$ & $8.3840 \cdot 10^{-2}$ & $2.1913 \cdot 10^{-2}$ \\
$N_{a} V_{d}$ & {$\left[\mathrm{~m}^{3}\right]$} & $4.6541 \cdot 10^{-2}$ & $1.8845 \cdot 10^{-1}$ & $4.6997 \cdot 10^{-2}$ \\
$N_{a} n V_{f}$ & {$\left[\mathrm{~m}^{3}\right]$} & $1.6393 \cdot 10^{-1}$ & $4.2274 \cdot 10^{-1}$ & $1.1049 \cdot 10^{-1}$ \\
\hline$\rho_{c}=\rho_{d}$ & {$\left[\frac{k_{g}}{3}\right]$} & $7.6027 \cdot 10^{+3}$ & $7.6027 \cdot 10^{+3}$ & $7.6027 \cdot 10^{+3}$ \\
$\rho_{f}^{a}$ & {$\left[\mathrm{~kg}^{3}\right]$} & $1.8149 \cdot 10^{+4}$ & $1.8900 \cdot 10^{+4}$ & $1.8900 \cdot 10^{+4}$ \\
\hline$m_{c}$ & {$[\mathrm{~kg}]$} & $1.0956 \cdot 10^{-2}$ & $7.0823 \cdot 10^{-2}$ & $9.2555 \cdot 10^{-2}$ \\
$m_{d}$ & {$[\mathrm{~kg}]$} & $7.0767 \cdot 10^{-1}$ & $2.8654 \cdot 10^{+0}$ & $3.5730 \cdot 10^{+0}$ \\
$m_{f}$ & {$[\mathrm{~kg}]$} & $5.5582 \cdot 10^{-2}$ & $8.8775 \cdot 10^{-1}$ & $1.1601 \cdot 10^{+0}$ \\
$N_{a} n m_{c}$ & {$[\mathrm{~kg}]$} & $4.9847 \cdot 10^{+2}$ & $6.3741 \cdot 10^{+2}$ & $1.6659 \cdot 10^{+2}$ \\
$N_{a} m_{d}$ & {$[\mathrm{~kg}]$} & $3.5384 \cdot 10^{+2}$ & $1.4327 \cdot 10^{+3}$ & $3.5730 \cdot 10^{+2}$ \\
$N_{a} n m_{f}$ & {$[\mathrm{~kg}]$} & $2.5290 \cdot 10^{+3}$ & $7.9898 \cdot 10^{+3}$ & $2.0882 \cdot 10^{+3}$ \\
\hline \hline
\end{tabular}

${ }^{a}$ fuel smear density is $85.0 \%$ of this theoretical density

Table 4.1: Volumes and Masses of Experimental Breeder Reactor II Clad, Duct and Fuel Components, Adapted from [44,53,54] 


\begin{tabular}{|c|c|c|}
\hline \multicolumn{2}{|c|}{ Parameter } & Shield \\
\hline$S_{a}=S_{d}$ & {$[m]$} & $5.8166 \cdot 10^{-2}$ \\
\hline$H_{\alpha}=H_{d}$ & {$[m]$} & $2.3324 \cdot 10^{+0}$ \\
\hline$N_{a}$ & {$[1]$} & 162 \\
\hline$V_{a}$ & {$\left[m^{3}\right]$} & $6.8340 \cdot 10^{-3}$ \\
\hline$N_{a} V_{a}$ & {$\left[m^{3}\right]$} & $1.1071 \cdot 10^{+0}$ \\
\hline$\rho_{a}$ & {$\left[\frac{\mathrm{kg}}{\mathrm{m}^{3}}\right.$} & $7.6027 \cdot 10^{+3}$ \\
\hline & {$\left[k_{S}\right.$} & $5.1957 \cdot 10^{+1}$ \\
\hline$N_{\mathrm{a}} m_{\mathrm{c}}$ & {$[\mathrm{kg}]$} & $8.4171 \cdot 10^{+3}$ \\
\hline
\end{tabular}

Table 4.2: Volumes and Masses of Experimental Breeder Reactor II Shield Component, Adapted from [44,53,54]

measure allowed for an initial burnup of 6.0nuclide - \% between 1969 and 1976. In later years, 8.0 nuclide - $\%$ on average were reached, with top burnups of 16.0 nuclide - \%. Also, the clad was changed to SS316 [44].

$E B R-I$ was converted to the sole use of "Mark-II" in October 1974, although first tests started in late 1969. "Mark-II" fuel elements and the latest "Mark-IIA" fuel elements are currently used in EBR-II [44].

\subsubsection{The Integral Fast Reactor}

\subsubsection{History \& Objectives}

In 1984, at the Argonne National Laboratory of the University of Chicago, Yoon I. Chang and Charles E. Till initiated the Integral Fast Reactor concept. It revives the idea behind $E B R-I I / F C F$ as it was installed during its 1964 to 1969 operation phase [5].

Parallel to the development of reactor and fuel cycle facility at $A N L$, Rockwell International (RI) and General Electric (GE) provided in 1988 with their reactor studies Sodium Advanced Fast Reactor (SAFR) [24] and Power Reactor Inherently Safe Module (PRISM) [42] own reactor designs for the IFR concept. 
Reactors and fuel cycle facilities according to the IFR concept will feature breeding and reprocessing. However, the reprocessing employed for $I F R$ is significantly different from the one used for $E B R-I I$, as stated in Section 4.4.

$E B R-I I$ will be equipped with an IFR core in 1990 and the $H F E F /$ South will be renamed back into $F C F$, then featuring the equipment necessary for $I F R$ reprocessing. In fact, $E B R$-II has already been used in 1986 to demonstrate the safety properties of the IFR concept [45]. The demonstration of breeding and reprocessing under the IFR concept in $E B R-I I / F C F$ will take place from 1991 to 1995 [5].

\subsubsection{Operation Schedule}

The IFR operation phase is defined by a nominal thermal power output of $900 M W_{t h}$. This corresponds to a neutron flux of $3.42 \cdot 10^{15} \frac{\mathrm{n}}{\mathrm{cm}^{2} \mathrm{~s}}$ for the driver section, $3.25 \cdot 10^{15} \frac{n}{\mathrm{~cm}^{2} s}$ for the internal breeder section, $1.66 \cdot 10^{15} \frac{\mathrm{n}}{\mathrm{cm}^{2} s}$ for the radial breeder section and $1.66 \cdot 10^{15} \frac{n}{\mathrm{~cm}^{2} \mathrm{~s}}$ for the reflector and shield section [35].

$25 \%$ of the core will be exchanged every year, after having been irradiated for four years at a capacity factor of 0.8 . This results in a total irradiation time of $292 d$ per year or $1,168 d$. The shield is assumed not to be exchanged in the reactor life time of $30 y$.

\subsubsection{Design}

IFR has a nominal thermal power output of $900 M W_{t h}$. This is converted to a nominal electrical power output of $350 \mathrm{MW}$, yielding a thermal plant efficiency of $\eta_{t h}=0.38$. The sodium at the core inlet has an average temperature of $357^{\circ} \mathrm{C}$ and heats up to an average temperature of $510^{\circ} \mathrm{C}$ at the core outlet [25]. 
The driver section is composed of 114 assemblies. 12 of those 114 assemblies are unfueled control assemblies. Scattered in the driver section are 37 internal breeder assemblies. The radial breeder section consists of 48 assemblies. The reflector and shield sections have 54 and 126 assemblies, respectively [34].

The driver assemblies contain 271 fuel elements. The internal and radial breeder assemblies contain only 169 fuel elements because of a larger fuel element diameter [34]. The shield and reflector assemblies are assumed to be solid blocks.

Tables 4.3 and 4.4 give volumes and masses of the clad, duct, fuel, reflector and shield components.

The IFR driver fuel contains approximately 10 weight $-\%$ of zirconium, which serves as a fuel stabilizer. The remainder of 90 weight $-\%$ consists of 69 weight $-\%$ uranium, 20 weight $-\%$ plutonium and 1 weight $-\%$ americium and curium. The uranium is chiefly ${ }^{238} U$ with ${ }^{235} U$ depleted to $0.2 n u c l i d e-$ $\%$. The plutonium is chiefly ${ }^{240} \mathrm{Pu}$ with ${ }^{239} \mathrm{Pu}$ enriched to 72.5 nuclide $-\%$. For the internal breeder and radial breeder fuel, natural or depleted uranium is chosen. The burnup is located in the $100,000 \frac{M W d}{t_{H M}}$ range $[34,51]$.

The clad and duct of all three core sections and the reflector and shield are made of $H T-9$. In addition, the shield contains $60 \mathrm{volume}-\%$ of $B_{4} C$. The approximate isotope and element composition of the fuel component and the element composition of the clad, duct, reflector and shield components are given in Appendix A.

\subsection{Fusion Reactors}

In this study, radioactive waste is identical with the solid fraction of reactor components. The identification of those components is facilitated, if the reactor is separated into inboard $(I B)$ and outboard $(O B)$ sections, as also mandated in Chapter 3.

A section contains a protective layer, a first wall, a breeder blanket, a 


\begin{tabular}{|c|c|c|c|c|}
\hline \multicolumn{2}{|c|}{ Parameter } & Driver & $\begin{array}{l}\text { Internal } \\
\text { Breeder }\end{array}$ & $\begin{array}{r}\text { Radial } \\
\text { Breeder }\end{array}$ \\
\hline$\overline{D_{c}}$ & {$[m]$} & $7.2390 \cdot 10^{-3}$ & $9.9568 \cdot 10^{-3}$ & $9.9568 \cdot 10^{-3}$ \\
\hline$\frac{P}{D_{s}}$ & {$[1]$} & 1.180 & 1.087 & 1.087 \\
\hline$t_{c}$ & [m] & $5.5880 \cdot 10^{-4}$ & $5.5880 \cdot 10^{-4}$ & $5.5880 \cdot 10^{-4}$ \\
\hline$S_{d}$ & {$[m]$} & $1.5014 \cdot 10^{-1}$ & $1.5014 \cdot 10^{-1}$ & $1.5014 \cdot 10^{-1}$ \\
\hline$t_{d}$ & {$[m]$} & $3.5560 \cdot 10^{-3}$ & $3.5560 \cdot 10^{-3}$ & $3.5560 \cdot 10^{-3}$ \\
\hline$D_{f}=D_{c}-2 t_{c}$ & {$[m]$} & $6.1214 \cdot 10^{-3}$ & $8.8392 \cdot 10^{-3}$ & $8.8392 \cdot 10^{-3}$ \\
\hline$H_{c}$ & {$[m]$} & $2.1336 \cdot 10^{+0}$ & $2.2352 \cdot 10^{+0}$ & $2.2352 \cdot 10^{+0}$ \\
\hline$H_{d}$ & {$[m]$} & $2.1336 \cdot 10^{+0}$ & $2.2352 \cdot 10^{+0}$ & $2.2352 \cdot 10^{+0}$ \\
\hline$H_{f}$ & {$[m]$} & $9.1440 \cdot 10^{-1}$ & $1.1176 \cdot 10^{+0}$ & $1.1176 \cdot 10^{+0}$ \\
\hline$N_{a}$ & {$[1]$} & 102 & 37 & 48 \\
\hline$n$ & [1] & 271 & 169 & 169 \\
\hline$\overline{V_{c}}$ & {$\left[m^{3}\right]$} & $2.5021 \cdot 10^{-5}$ & $3.6877 \cdot 10^{-5}$ & $3.6877 \cdot 10^{-5}$ \\
\hline$V_{d}$ & {$\left[\mathrm{~m}^{3}\right]$} & $3.9460 \cdot 10^{-3}$ & $4.1339 \cdot 10^{-3}$ & $4.1339 \cdot 10^{-3}$ \\
\hline$V_{f}$ & {$\left[m^{3}\right]$} & $2.6911 \cdot 10^{-5}$ & $6.8581 \cdot 10^{-5}$ & $6.8581 \cdot 10^{-5}$ \\
\hline$N_{a} n V_{c}$ & {$\left[m^{3}\right]$} & $6.9164 \cdot 10^{-1}$ & $2.3059 \cdot 10^{-1}$ & $2.9915 \cdot 10^{-1}$ \\
\hline$N_{a} V_{d}$ & {$\left[\mathrm{~m}^{3}\right]$} & $4.0249 \cdot 10^{-1}$ & $1.5296 \cdot 10^{-1}$ & $1.9843 \cdot 10^{-1}$ \\
\hline$N_{a} n V_{f}$ & {$\left[m^{3}\right]$} & $7.4387 \cdot 10^{-1}$ & $4.2884 \cdot 10^{-1}$ & $5.5633 \cdot 10^{-1}$ \\
\hline$\rho_{c}=\rho_{d}$ & {$\left[\frac{\mathrm{kg}}{\mathrm{m}^{3}}\right]$} & $7.7321 \cdot 10^{+3}$ & $7.7321 \cdot 10^{+3}$ & $7.7321 \cdot 10^{+3}$ \\
\hline$\rho_{f}^{a}$ & {$\left[\frac{\mathrm{kg}}{\mathrm{m}^{3}}\right]$} & $1.5701 \cdot 10^{+4}$ & $1.5740 \cdot 10^{+4}$ & $1.5740 \cdot 10^{+4}$ \\
\hline$m_{c}$ & {$[\mathrm{~kg}]$} & $1.9347 \cdot 10^{-1}$ & $2.8514 \cdot 10^{-1}$ & $2.8514 \cdot 10^{-1}$ \\
\hline$m_{d}$ & {$[\mathrm{~kg}]$} & $3.0511 \cdot 10^{+1}$ & $3.1964 \cdot 10^{+1}$ & $3.1964 \cdot 10^{+1}$ \\
\hline$m_{f}$ & {$[k g]$} & $3.1690 \cdot 10^{-1}$ & $8.0962 \cdot 10^{-1}$ & $8.0962 \cdot 10^{+3}$ \\
\hline$N_{a} n m_{c}$ & {$[\mathrm{~kg}]$} & $5.3478 \cdot 10^{+3}$ & $1.7830 \cdot 10^{+3}$ & $2.3131 \cdot 10^{+3}$ \\
\hline$N_{\mathrm{a}} m_{d}$ & {$[k g]$} & $3.1121 \cdot 10^{+3}$ & $1.1827 \cdot 10^{+3}$ & $1.5343 \cdot 10^{+3}$ \\
\hline$N_{a} n m_{f}$ & {$[k g]$} & $8.7599 \cdot 10^{+3}$ & $5.0626 \cdot 10^{+3}$ & $6.5677 \cdot 10^{+3}$ \\
\hline
\end{tabular}

${ }^{a}$ fuel smear density is $75.0 \%$ of this theoretical density

Table 4.3: Volumes and Masses of Integral Fast Reactor Clad, Duct and Fuel Components, Adapted from [14,17,25,34,35,51] 


\begin{tabular}{|lr|r|r|}
\hline \hline \multicolumn{2}{|c|}{ Parameter } & Reflector & Shield \\
\hline$S_{a}=S_{d}$ & {$[\mathrm{~m}]$} & $1.5014 \cdot 10^{-1}$ & $1.5014 \cdot 10^{-1}$ \\
$H_{a}=H_{d}$ & {$[\mathrm{~m}]$} & $2.2352 \cdot 10^{+0}$ & $2.2352 \cdot 10^{+0}$ \\
\hline$N_{a}$ & {$[1]$} & 54 & 126 \\
\hline$V_{a}$ & {$\left[\mathrm{~m}^{3}\right]$} & $4.3635 \cdot 10^{-2}$ & $4.3635 \cdot 10^{-2}$ \\
$N_{a} V_{a}$ & {$\left[\mathrm{~m}^{3}\right]$} & $2.3563 \cdot 10^{+0}$ & $5.4980 \cdot 10^{+0}$ \\
\hline$\eta_{H T 9}$ & {$[1]$} & 0.816 & 0.219 \\
$\eta_{B_{4} C}$ & {$[1]$} & - & 0.597 \\
$\rho_{H T 9}$ & {$\left[\frac{\mathrm{kg}}{\mathrm{m}^{3}}\right]$} & $7.7321 \cdot 10^{+3}$ & $7.7321 \cdot 10^{+3}$ \\
$\rho_{B_{4} C}^{a}$ & {$\left[\frac{\mathrm{kg}^{3}}{\mathrm{~m}^{3}}\right]$} & - & $2.5200 \cdot 10^{+3}$ \\
$\rho_{a}$ & {$\left[\frac{\mathrm{m}^{3}}{\mathrm{~m}^{3}}\right]$} & $6.3094 \cdot 10^{+3}$ & $2.5509 \cdot 10^{+3}$ \\
\hline$m_{a}$ & {$[\mathrm{~kg}]$} & $2.7531 \cdot 10^{+2}$ & $1.1131 \cdot 10^{+2}$ \\
$N_{a} m_{a}$ & {$[\mathrm{~kg}]$} & $1.4867 \cdot 10^{+4}$ & $1.4025 \cdot 10^{+4}$ \\
\hline \hline
\end{tabular}

${ }^{a} B_{4} C$ smear density is $57.0 \%$ of this theoretical density

Table 4.4: Volumes and Masses of Integral Fast Reactor Reflector and Shield Components, Adapted from [14,17,25,34,35,51]

back wall and/or manifold, a shield, a vacuum vessel, and toroidal coils. The inboard section in addition has an ohmic heating coil, which is referred to in this study as the poloidal coil. Coolant is gaseous or liquid at the time of discharge from the reactor and therefore is not considered.

Thus, protective layer, first wall, breeder blanket, back wall and/or manifold, shield, vacuum vessel, toroidal coils and poloidal coil of the two sections shall be considered as components in this study. Clearly, these components are defined by distinct functions within a reactor. Note, that in contrast to the components of a fast fission reactor, each component typically consists of more than one material.

Two components not considered in this study are the divertor and the limiter, which have a minute volume in comparison to the rest of the components. Nevertheless, those components can have a very high activation. Also, for the reactors considered in this study, there was no proper information available on the divertor or limiter design.

Two further components that shall be mentioned here are the cryostat 
coolant and the cryostat vessel, which are present only in cryogenically cooled reactors. Their activation in general is negligible.

ITER and RAFHT are reactors of the Tokamak type and employ the $D-T$ fusion reaction $[19,21]$. Typical neutron energies in the reactor are at a maximum of $14 \mathrm{MeV}$. The typical neutron flux is at $10^{15} \frac{n}{\mathrm{~cm}^{2} \mathrm{~s}}$, although it can be as low as $10^{2} \frac{n}{\mathrm{~cm}^{2} \mathrm{~s}}$.

\subsubsection{The International Thermonuclear Experimen- tal Reactor}

\subsubsection{History \& Objectives}

In 1987, the Director General of the International Atomic Energy Agency (IAEA) invited representatives of the United States, Europe, the Soviet Union and Japan to initiate a more invloved international cooperation on fusion energy, eventually resulting in a proposal for ITER $[21,23]$.

Under the auspices of the IAEA, definition activities for ITER began in 1988, followed by design activities in 1989 and 1990 [23].

The first objective of ITER is the expansion of the international cooperation on fusion energy, especially among the four parties mentioned above. The second objective of ITER is to demonstrate that the fusion reaction can be employed to generate net energy. Ignition, extended burn and steady burn of the plasma are milestones on this way $[21,23]$.

Only then the build-up of operating experience in terms of designing, building and maintaining a fusion reactor can be considered as useful, including the demonstration of a self-sufficient closed fuel cycle. It is interesting to note in the context of radioactive waste, that the above objectives are subjected to an "environmentally acceptable operation" [23]. 


\subsubsection{Operation Schedule}

Two ITER operation phases have to be distinguished. The Physics Phase concentrates on plasma performance and the Technology Phase focuses on component performance [21].

The major differences between both phases consist in the different plasmas used, the different number of plasma shots and the different plasma burn times. Physics phase and technology phase will have one set of components [21].

The physics phase provides a duration of about six years and a number of approximately 15000 shots. Those six years are divided into three different stages, depending on the plasma used in each stage. The stages are given in Table 4.5.

\begin{tabular}{|l|c|c|c|}
\hline \hline \multicolumn{3}{|c|}{ Stage } & Shots \\
\hline Number & Plasma Type & Duration & Number \\
\hline 1 & ${ }_{1}^{1} H$ & $2.5 y$ & 6000 \\
2 & ${ }_{1}^{2} H+{ }_{2}^{3} H e$ & $1.0 y$ & 2000 \\
3 & ${ }_{1}^{3} H++_{1}^{2} H$ & $2.5 y$ & 7000 \\
\hline
\end{tabular}

Table 4.5: Stages of ITER Physics Phase, Adapted from [21,22]

Apparently only the third stage will contribute significantly to any activation of reactor components. This third stage is divided into three substages, which are given in Table 4.6. The neutron first wall load for all plasma shots in these substages is assumed to be $1.0 \frac{\mathrm{MW}}{\mathrm{m}^{2}}[21,22]$.

The technology phase provides a duration of about ten years without a number of plasma shots specified. However, an attempt to reach a steady state plasma will be made. For this study, it is assumed that the maximum achievable performance of ITER with a steady state plasma is a total of $0.5 y$ operation per year at $1.0 \frac{\mathrm{MW}}{\mathrm{m}^{2}}$. Furthermore this study assumes that this will be the case only for the last two years of the $10 y$ technology phase. Assuming a linear increase of the performance by a total of $0.1 y$ every two 


\begin{tabular}{|l|c|c|c|c|}
\hline \multicolumn{3}{|c|}{ Substage } & \multicolumn{2}{c|}{ Plasma Shots } \\
\hline Number & Plasma Type & Duration & Number & Duration \\
\hline 1 & ${ }_{1}^{3} H-{ }_{1}^{2} H$ & $0.83 y$ & 3000 & $20 s$ \\
2 & ${ }_{1}^{3} H-{ }_{1}^{2} H$ & $0.83 y$ & 2000 & $200 s$ \\
3 & ${ }_{1}^{3} H-l_{1}^{2} H$ & $0.83 y$ & 2000 & $1000 s$ \\
\hline \hline
\end{tabular}

Table 4.6: Substages of Stage 3 of ITER Physics Phase, Adapted from $[21,22]$

years, the technology phase starts out with a total of $0.1 y$ operation per year for the first two years. Each two year period is then considered a stage. This scheme for the technology phase represents an approximation that was made solely for the purpose of this study. Although it is based upon references, it can not be found in this form in any of these references.

Since the third stage of the physics phase will have essentially the same components as the technology phase, a total duration of $12.5 y$ can be concluded for the two operation phases.

With the outlined intensity and duration of the irradiation for the physics and technology phase, an average neutron first wall load as given in Table 4.7 can be derived, where the duration of all plasma shots of a stage or substage is given as the percentage of the duration of the corresponding stage or substage.

The total neutron first wall fluence for the combined operation phases is then given as $3.0778 \frac{\mathrm{MWa}}{\mathrm{m}^{2}}$. These figures result in $3.1167 \% F P D$ and $30.000 \% F P D$ for the physics and technology phase, respectively, where FPD means full power day. No reactor component is assumed to be exchanged during the $12.5 y$. However, at least the protective layer specified in Section 4.3.1.3 will be exchanged frequently. It shows only low activation and therefore does not upset the comparison. 


\begin{tabular}{|c|c|c|c|}
\hline Duration & $\begin{array}{l}\text { Nominal Neutron } \\
\text { First Wall Load }\end{array}$ & $\begin{array}{r}\text { Duration } \\
\text { of Shots }\end{array}$ & $\begin{array}{l}\text { Average Neutron } \\
\text { First Wall Load }\end{array}$ \\
\hline $0.83 y$ & $1.0 \frac{M W}{x^{2}}$ & $0.23 \%$ & $2.3 \cdot 10^{-3} \frac{M W}{s^{2}}$ \\
\hline $0.83 y$ & $1.0 \frac{m^{2} W}{m^{2}}$ & $1.52 \%$ & $1.5 \cdot 10^{-2} \frac{\mathrm{m}^{2} W}{\mathrm{~m}^{2}}$ \\
\hline $0.83 y$ & $1.0 \frac{M W}{m^{2}}$ & $7.60 \%$ & $7.6 \cdot 10^{-2} \frac{3 W}{m^{2}}$ \\
\hline $2.00 y$ & $1.0 \frac{\mathrm{m} W}{\mathrm{~m}^{2}}$ & $10.00 \%$ & $1.0 \cdot 10^{-1} \frac{M W}{m^{2}}$ \\
\hline $2.00 y$ & $1.0 \frac{M W}{m^{2}}$ & $20.00 \%$ & $2.0 \cdot 10^{-1} \frac{M W}{m^{2}}$ \\
\hline $2.00 y$ & $1.0 \frac{m_{W}}{\pi^{2}}$ & $30.00 \%$ & $3.0 \cdot 10^{-1} \frac{m^{2} W}{m^{2}}$ \\
\hline $2.00 y$ & $1.0 \frac{\mathrm{m} W}{\mathrm{~m}^{2}}$ & $40.00 \%$ & $4.0 \cdot 10^{-1} \frac{\mathrm{m} W}{\mathrm{~m}^{2}}$ \\
\hline $2.00 y$ & $1.0 \frac{\mathrm{d} W}{\mathrm{~m}^{2}}$ & $50.00 \%$ & $5.0 \cdot 10^{-1} \frac{\mathrm{d} W}{\mathrm{~m}^{2}}$ \\
\hline
\end{tabular}

Table 4.7: Average Neutron First Wall Load of ITER Operation Phases

\subsubsection{Design}

ITER has a nominal fusion power output of $830 M W_{f}$. This could theoretically be converted to approximately $1,000 \mathrm{M} W_{\text {th }}$ and about $350 \mathrm{MW}$ with an assumed thermal plant efficiency of $\eta_{t h}=0.35$. However, ITER is not designed to deliver any electrical power output. Toroidal and poloidal coils are cooled by liquid helium, all other components are cooled by low-pressure water [21].

Reference [21] contains detailed drawings of the major ITER dimensions. The radial build of the components first wall, breeder blanket, back wall, shield and toroidal coils and the materials thereof were adapted from Reference [11]. The radial build of the materials given therein did not match the major ITER dimensions and hence this study modified the radial build of the materials to make it fit into the major ITER dimensions. The major radius is $R=6 \mathrm{~m}$, the minor radius $a=2.15 \mathrm{~m}$, with an elongation of $\kappa=2.5[21]$.

The protective layer consists of carbon fiber tiles for the inboard section. Those may be cooled by conduction or radiation. A sprayed layer of tungsten is also considered. The outboard section most likely has no protective layer [21]. 
First wall, back wall and breeder blanket are integrated. The breeder blanket of the inboard section consists of a sandwich of one layer of breeder material between two layers of multiplier material. The breeder blanket of the outboard section consists of two such sandwiches. Note, that the multiplier material not only multiplies the neutron flux in order to yield a higher tritium breeding ratio but also serves as a thermal insulation in order to reduce the temperature of the coolant $[11,21]$.

The breeder blankets have not been designed to produce the tritium necessary for a self-sufficient closed fuel cycle, but will operate as realistically as possible under power reactor conditions. The tritium removal from the breeder blankets is effected by means of helium purging [21].

The shield has a steel shield and a lead shield as its subcomponents. The toroidal and poloidal coils are composites of superconducting and normal conducting conductors. The same is assumed for the ohmic heating or poloidal coil.

Tables 4.8 and 4.9 give volumes and masses of the components. The height of the reactor components has been assumed as $H_{c}=10 \mathrm{~m}$.

ITER has 16 toroidal coils which do not form a cylindrical layer at all. The volume derived for the toroidal coils by a cylindrical approximation therefore was multiplied by 0.86 for the inboard section and 0.21 for the outboard section. Those factors can be obtained by comparing the actual shape of the coils to the cylindrical layer approximation.

The breeder material for ITER is solid $\mathrm{Li}_{2} \mathrm{O}$ with the lithium enriched to 90 nuclide $-\%^{8} \mathrm{Li}$. Due to the high enrichment, there is no need to utilize $\mathrm{Li}_{2} \mathrm{O}$ thicknesses greater than $10 \mathrm{~mm}$ in order to achieve a sufficient tritium breeding ratio. The multiplier material is $\mathrm{Be}$. The $\mathrm{BeO}$ impurity in $B e$ is less than 1.0 weight $-\%$, so it is relatively pure beryllium. Structure material serves as a breeder blanket stabilizer $[11,21]$.

All component structure is made of stainless steel $S S 316$. The lead shield features $\mathrm{B}_{4} \mathrm{C}$ as a neutron absorber. Superconducting and normal- 


\begin{tabular}{|c|c|c|c|c|}
\hline Parameter & Component & Inboard & Outboard & $\overline{\text { Total }}$ \\
\hline \multirow[t]{10}{*}{$\overline{V_{c} / \eta_{c}}\left[\mathrm{~m}^{3}\right]$} & Protective Layer & 4.6 & $=$ & 4.6 \\
\hline & First Wall & 1.2 & 2.6 & $\overline{3.8}$ \\
\hline & Breeder Blanket & 30.6 & 201.2 & 231.8 \\
\hline & Back Wall & 15.4 & 27.4 & 42.8 \\
\hline & Steel Shield & 48.2 & 405.1 & 453.3 \\
\hline & Lead Shield & 6.0 & 17.9 & 23.9 \\
\hline & Vacuum Vessel & $\overline{56.9}$ & 182.1 & $\overline{239.0}$ \\
\hline & Toroidal Coils & 89.7 & 160.6 & 250.3 \\
\hline & Poloidal Coil & 68.7 & - & 68.7 \\
\hline & Reactor & 321.3 & 996.9 & 1318.2 \\
\hline \multirow[t]{10}{*}{$m_{c}$} & Protective Layer & 10.4 & - & 10.4 \\
\hline & First Wall & $\overline{9.1}$ & 20.6 & 29.7 \\
\hline & Breeder Blanket & 64.2 & 409.1 & 473.3 \\
\hline & Back Wall & 121.8 & 216.1 & 337.9 \\
\hline & Steel Shield & 266.6 & 2326.9 & 2593.5 \\
\hline & Lead Shield & 52.5 & 155.2 & 207.7 \\
\hline & Vacuum Vessel & 449.7 & 1438.5 & 1888.2 \\
\hline & Toroidal Coils & $\overline{736.7}$ & 1324.0 & 2060.7 \\
\hline & Poloidal Coil & 565.3 & - & 565.3 \\
\hline & Reactor & 2276.3 & 5890.4 & 8166.7 \\
\hline
\end{tabular}

Table 4.8: Volumes and Masses of International Thermonuclear Experimental Reactor Components, Adapted from [11,21] 


\begin{tabular}{|c|c|c|c|c|}
\hline Parameter & Component & Inboard & Outboard & Total \\
\hline \multirow[t]{10}{*}[\frac{t}{m^{3}}]{} & Protective Layer & 2.3 & - & $\overline{2.3}$ \\
\hline & First Wall & 7.6 & $\overline{7.9}$ & 7.8 \\
\hline & Breeder Blanket & 2.1 & 2.0 & 2.0 \\
\hline & Back Wall & 7.9 & $\overline{7.9}$ & 7.9 \\
\hline & Steel Shield & 5.5 & $\overline{5.7}$ & 5.7 \\
\hline & Lead Shield & 8.8 & 8.7 & 8.7 \\
\hline & Vacuum Vessel & 7.9 & $\overline{7.9}$ & 7.9 \\
\hline & Toroidal Coils & 8.2 & $\overline{8.2}$ & 8.2 \\
\hline & Poloidal Coil & 8.2 & - & 8.2 \\
\hline & Reactor & 7.1 & 5.9 & 6.2 \\
\hline \multirow[t]{10}{*}{$\eta_{c}$} & Protective Layer & 100.0 & 二 & 100.0 \\
\hline & First Wall & 100.0 & 100.0 & 100.0 \\
\hline & Breeder Blanket & 96.3 & 98.2 & 97.9 \\
\hline & Back Wall & 100.0 & 100.0 & 100.0 \\
\hline & Steel Shield & 65.7 & 68.8 & 68.50 \\
\hline & Lead Shield & 100.0 & 100.0 & 100.0 \\
\hline & Vacuum Vessel & 100.0 & 100.0 & 100.0 \\
\hline & Toroidal Coils & 80.0 & 80.0 & 80.0 \\
\hline & Poloidal Coil & 80.0 & $=$ & 80.0 \\
\hline & Reactor & 84.6 & 83.7 & 83.9 \\
\hline
\end{tabular}

Table 4.9: Weight Densities and Solid Volume Fractions of International Thermonuclear Experimental Reactor Components, Adapted from [11,21] 
conducting conductors are made of $\mathrm{Nb}_{3} \mathrm{Sn}$ and $\mathrm{Cu}$ as stabilizer, respectively [21].

The approximate material composition of the components as well as their radial build is given in Appendix A.

Note that the ITER design presented here does not necessarily represent the final ITER design, since work on ITER was continued after completion of this study. However, the present and the final design should not differ substantially. Also, for some reactor components, several alternatives exist, one of which had to be chosen for this study.

\subsubsection{The Reduced Activation Ferrite Helium Toka- mak}

\subsubsection{History \& Objectives}

In 1989, the Senior Committee on Environmental, Safety and Economic Aspects of Magnetic Fusion Energy (ESECOM) conducted a study to assess fusion reactors with respect to safety, economy and environmental hazard potential [19].

Several fusion reactors were considered. The breeder blankets of most of those reactors stem from Reference [43], which is mentioned in Reference [19]. This holds true also for the breeder blanket of RAFHT. It was ranked at the top of the breeder blankets considered in Reference [43]. This and the fact that Reference [19] assigns it the highest potential of realization were the reasons for $R A F H T$ being chosen for this study.

\subsubsection{Operation Schedule}

The RAFHT operation phase is defined by a nominal thermal power output of $3,648 M W_{t h}$. The neutron first wall load is given as $3.18 \frac{\mathrm{MW}}{\mathrm{m}^{2}}$.

All components will be exchanged after 6 years of operation. However, the shield will not be exchanged during the reactor life time of $30 y$. 


\subsubsection{Design}

RAFHT has a nominal fusion power output of $3,027 M W_{f}$. This is converted to $3,648 \mathrm{M} W_{\text {th }}$ and $1,200 \mathrm{MW}$ with a calculated thermal plant efficiency of $\eta_{t h}=0.33$. All components are cooled by gaseous helium, except for the shield and presumably the toroidal and poloidal coils, which are cooled by water [19]. The radial build of the components is given in Reference [19], the major radius is $R=6.07 \mathrm{~m}$, the minor radius $a=1.52 \mathrm{~m}$, with an elongation of $\kappa=2.5$. Information on the vacuum vessel, the toroidal coils and the poloidal coil was not available.

Table 4.10 gives volumes and masses of the components. The height of the components has been assumed as $H_{c}=10 \mathrm{~m}$.

Only insufficient information on the materials used in $R A F H T$ is available. However, the breeder material for $\mathrm{RAFHT}$ is solid $\mathrm{Li}_{2} \mathrm{O}$ with the assumption of lithium being enriched to 90 nuclide $-\%{ }^{8} \mathrm{Li}$. Structure material serves as a breeder blanket stabilizer. All component structure is made of Reduced Activation Material (RAM), i.e. stainless steel RAF. The $R A M F e C r V$ is used for the shield [19].

The approximate material composition of the components as well as their radial built is given in Appendix A.

\subsection{Radioactive Waste Processing for Fast Fission Reactors}

\subsubsection{EBR-II and IFR Pyroprocesses}

The destinations of $E B R-I I$ and IFR require two different types of fuel. The one for the driver section has to have a high ${ }^{235} U$ content in the case of $E B R-I I$ and a high non- ${ }^{238} U$ actinide content in the case of $I F R$. The fuel for the breeder sections has to have a high ${ }^{238} U$ content. The distinction is important to notice, since spent fuel from both sections enters the same 


\begin{tabular}{|c|c|c|c|c|}
\hline Parameter & Component & Inboard & Outboard & Total \\
\hline \multirow{5}{*}{$V_{c} / \eta_{c} \quad\left[m^{3}\right]$} & First Wall & 16.5 & 29.3 & 45.8 \\
\hline & Breeder Blanket & $\overline{145.2}$ & 289.7 & 434.9 \\
\hline & Manifold & 50.6 & 117.3 & 167.9 \\
\hline & Steel Shield & 62.2 & 166.7 & 228.9 \\
\hline & Reactor & 274.5 & 603.0 & $\overline{877.5}$ \\
\hline \multirow[t]{5}{*}{$m_{c} \quad[t]$} & First Wall & 19.1 & 34.0 & 53.1 \\
\hline & Breeder Blanket & 367.6 & 740.0 & 1107.6 \\
\hline & Manifold & 89.0 & 206.4 & 295.4 \\
\hline & Steel Shield & 396.0 & 1061.7 & 1457.7 \\
\hline & Reactor & 871.7 & 2042.1 & 2913.8 \\
\hline \multirow[t]{5}{*}{$\rho_{c} \quad\left[\frac{t}{m^{3}}\right]$} & First Wall & 1.2 & 1.2 & 1.2 \\
\hline & Breeder Blanket & 2.5 & 2.6 & 2.5 \\
\hline & Manifold & 1.8 & 1.8 & 1.8 \\
\hline & Steel Shield & 6.4 & 6.4 & 6.4 \\
\hline & Reactor & 3.2 & $\overline{3.4}$ & $\overline{3.3}$ \\
\hline \multirow{5}{*}{$\eta_{c}$} & First Wall & 12.0 & 12.0 & 12.0 \\
\hline & Breeder Blanket & 89.6 & 89.7 & 89.6 \\
\hline & Manifold & 20.0 & 20.0 & 20.0 \\
\hline & Steel Shield & 80.0 & 80.0 & 80.0 \\
\hline & Reactor & 69.9 & 69.7 & 69.8 \\
\hline
\end{tabular}

Table 4.10: Volumes and Masses, Weight Densities and Solid Volume Fractions of Reduced Activation Ferrite Helium Tokamak Components, Adapted from [19] 
EBR-II and IFR High Level Radioactive Waste Pyroprocess, but in the case of $E B R$-II the products are used for the fabrication of fuel for the driver section only, while in the case of $I F R$ the products are used for fabrication of fuel for both the driver and breeder section. EBR-II actinides contain virtually only ${ }^{235} U$ and ${ }^{238} U$, while $I F R$ actinides feature the full spread $[5,44]$.

The EBR-II pyroprocess comprises two subprocesses, the IFR pyroprocess comprises three subprocesses. The subprocesses of melt-refining for $E B R-I I$ and electro-refining for IFR separate the major part of fission and activation products from the major part of actinides. The partition between fission and activation products on the one hand and actinides on the other hand, however, is not complete; so small amounts of the latter group will accompany the former two groups and vice versa $[5,44]$.

This necessitates the subprocesses of recovering for $E B R-I I$ and purifying and extracting and stripping for IFR. The subprocess of recovering removes the small share of actinides from the fission and activation products. The subprocess of purifying removes the small share of fission and activation products from the actinides and the subprocess of extracting and stripping has the same function as the one of recovering. For $E B R-I I$, there is no need to remove the small share of fission and activation products from the actinides by a subprocess equivalent to the $I F R$ subprocess of purifying, because this small share serves as a fuel stabilizer. For $I F R$, such a fuel stabilizer has to be added extra $[5,44]$.

The major part of the fission and activation products is destined for the $R S W F$ in the case of $E B R-I I$ and for deep repositories in the case of $I F R$. The major part of the actinides is refabricated into the aforesaid driver and/or breeder fuel $[5,44]$.

Gaseous fission products are extracted throughout the entire process via filters and treated separately. Tritium, krypton and xenon dominate those gaseous fission products $[5,44]$. 
In Sections 4.4.3 and 4.4.4, a somewhat simplyfied and idealized description of the $E B R-I I$ and $I F R$ pyroprocesses is presented. Although there are some modifications and specific considerations as to the design and the operation of an actual pyroprocessing plant $[5,44]$ that can not be regarded in the frame of this study, the essential technique of $E B R-I I$ and $I F R$ pyroprocessing is explained in some detail. Also, it is suitable to first review some of the chemistry that forms the basis of the pyroprocesses. This review is given in Section 4.4.2.

\subsubsection{Basic Chemistry}

The chemical foundation of the pyroprocesses is the fact that elements of the chemical classification groups as listed in Section 1.2 have different potentials or affinities for oxidation and reduction. This is qualitatively reflected in Table 4.11 .

\begin{tabular}{|l|c|c|}
\hline $\begin{array}{l}\text { Classification } \\
\text { Group }\end{array}$ & $\begin{array}{c}\text { Oxidation } \\
\text { Potential }\end{array}$ & $\begin{array}{c}\text { Reduction } \\
\text { Potential }\end{array}$ \\
\hline Alkaline Metals & very high & very low \\
Alkaline Earth Metals & high & low \\
Lanthanides & high to medium & low to medium \\
Actinides & medium to low & medium to high \\
General Metals & low & high \\
Noble Metals & very low & very high \\
\hline
\end{tabular}

Table 4.11: Oxidation and Reduction Potentials, Adapted from [3,5]

Oxidation potential shall be understood as the affinity toward the reaction $M_{i} \rightarrow M_{i}^{j+}+j e^{-}$, releasing metal ions in a reaction with an oxidant. Reduction potential denotes the affinity toward the reaction $\mathrm{Mi}_{i}^{j+}+j e^{-} \rightarrow$ $M_{i}$, accepting electrons in a reaction with a reductant. Hence oxidation is the affinity for giving electrons and reduction is the affinity for taking electrons. An oxidant takes electrons and thus undergoes reduction, a reduc- 
tant gives electrons and thus undergoes oxidation. Consistently, reactions at anodes are oxidations, reactions at cathodes are reductions [3].

In electrolytes, a certain fraction of metal compounds will be dissociated into metal ions and ions of the compound partner and a certain fraction of metal atoms will be dissociated into metal ions and electrons. Metal ions usually have positive charge, compound partner ions usually have negative charge. The degree of dissociation depends on the oxidation potential of the metal. High oxidation potentials result in a low degree of dissociation, low oxidation potentials in a high degree.

A quantitative assessment of oxidation and reduction potentials can be done via comparison of the free energy of formation in standard state, $\Delta G_{f}^{o}$, of an oxidation product. It is essentially the difference between the free energies of products and reactants of the oxidation-reduction (REDOX) reaction. Oxidation will occur, if this free energy of formation is negative, reduction will occur, if it is positive. This is a direct consequence of the fact that a system reduces its free energy if it is given the chance to do so. The affinity for oxidation or reduction increases with increasing absolute value of the free energy of formation, and a zero free energy of formation means no reaction takes place because equilibrium is reached [3].

This scheme, however, only holds true if there is no external energy source or sink. A source will accelerate the corresponding reaction, a sink will decelerate it. Typical sources of energy in chemical processes are elevated temperatures or electrical potentials [3]. The magnitude of the electrical potential itself and the interface area between electrolyte and electrodes are important factors in the latter case. The oxidation potential can also be influenced by the effect of passivation. A surface layer of material with very low oxidation potential (e.g. oxides) formed by a material with relatively high oxidation potential serves as protection against further oxidation of this material.

Free energies of formation for the chlorine oxidation products (chlorides) 
of some of the elements of the chemical classification groups are provided in Table 4.12. A similar table can be composed, e.g. for oxygen oxidation products, with the order only slightly changed.

\subsubsection{EBR-II Pyroprocess}

\subsubsection{Melt-refining Process}

The melt-refining process requires the mechanical separation of fuel from clad. The spent driver and breeder fuel with adhering thermal bond sodium is then melted in a crucible at about $1,400^{\circ} \mathrm{C}$ [44]. The melt can be considered an electrolyte. Due to their high oxidation potential, alkali, alkaline earth and rare earth metal atoms present in the spent fuel will undergo almost no dissociation in the melt, while actinide, general and noble metal atoms feature a low oxidation potential and therefore will exhibit a considerable degree of dissociation. The oxidant in the primitive melt-refining process is oxygen. Addition of $\mathrm{ZrO}_{2}$ to the melt does release a sufficient amount of oxygen ions because of the low oxidation potential of the general metal $Z r$. Depending on their oxidation potentials, most metal atoms present in the melt will now with different intensities form metal oxides that partly dissociate into metal and oxygen ions subsequently.

High oxidation potential metals will almost entirely form metal oxides with only a minor share remaining as metal atoms and very few metal oxides dissociating into ions. Low oxidation potential metals will almost entirely remain as metal atoms with only a minor share forming metal oxides and very many metal oxides dissociating into ions.

This effects the separation of the actinides and the major part of the general and noble metals of the fission and activation products from the major part of the alkali, alkaline earth and rare earth metals of the fission and activation products. The result is the formation of a dross of alkali, alkaline earth and rare earth metal oxides, contaminated with a few actinide, 


\begin{tabular}{|l|r|}
\hline \hline Oxidation Product & $\Delta \mathrm{G}_{\mathrm{f}}$ \\
\cline { 2 - 2 } & {$\left[\frac{\mathrm{J}}{\mathrm{kq}}\right]$} \\
\hline $\mathrm{BaCl}_{2}$ & 367773.6 \\
$\mathrm{CsCl}$ & 367355.2 \\
$\mathrm{RbCl}$ & 364008.0 \\
$\mathrm{KCl}$ & 362752.8 \\
$\mathrm{SrCl} l_{2}$ & 354384.8 \\
$\mathrm{LiCl}$ & 345180.0 \\
$\mathrm{NaCl}$ & 339322.4 \\
$\mathrm{CaCl}$ & 337648.8 \\
$\mathrm{LaCl}_{3}$ & 293716.8 \\
$\mathrm{PrCl}_{3}$ & 288696.0 \\
$\mathrm{CeCl}_{3}$ & 287022.4 \\
$\mathrm{NdCl}$ & 284093.6 \\
$\mathrm{YCl}$ & 272378.4 \\
$\mathrm{CmCl}_{3}$ & 267776.0 \\
$\mathrm{PuCl}_{3}$ & 261081.6 \\
$\mathrm{MgCl}_{2}$ & 259826.4 \\
$\mathrm{NpCl}_{3}$ & 243090.4 \\
$\mathrm{UCl} l_{2}$ & 230538.4 \\
$\mathrm{ZrCl}_{2}$ & 194974.4 \\
$\mathrm{CdCl}_{2}$ & 135143.2 \\
$\mathrm{FeCl}_{2}$ & 122172.8 \\
$\mathrm{NbCl}_{5}$ & 111712.8 \\
$\mathrm{MoCl}_{2}$ & 70291.2 \\
$\mathrm{TcCl}$ & 46024.0 \\
$\mathrm{RhCl}_{3}$ & 41840.0 \\
$\mathrm{PdCl}_{2}$ & 37656.0 \\
$\mathrm{RuCl}_{3}$ & 28869.6 \\
\hline \hline
\end{tabular}

Table 4.12: Free Energy of Formation at $500^{\circ} \mathrm{C}$, Adapted from [5] 
general and noble metal oxides. Most actinide, general and noble metal atoms will remain in the melt, contaminated by a few alkali, alkaline earth and rare earth metal atoms [44].

Subsequently, the thus purified melt is mechanically separated from the dross; during this process, minor parts of the actinide, general and noble metal atoms are mechanically retained by the dross and hence will contaminate it in addition to the actinide, general and noble metal oxides. The contaminated dross is called skull. New fuel can be refabricated directly from the purified melt. General and noble metals in the purified melt constitute desirable fuel alloy elements. The fuel typically shows a composition of $95 w e i g h t-\%$ actinides and 5 weight $-\%$ general and noble metals, where the latter $5 w e i g h t-\%$ are referred to as fissium [44].

\subsubsection{Recovering Process}

The recovery process consists of the two steps of skull oxidation and skull reclamation. These steps allow the removal of actinides including a major part of the general and noble metals from the skull. The skull represents a considerable resource of actinides, so it is reasonable to contemplate this removal [44].

However, only the skull oxidation step was conducted at the FCF. The skull reclamation step was demonstrated only in a laboratory arrangement. Therefore, the product of the skull oxidation step was directly shipped to the ICPP for storage [44].

4.4.3.2.1 Skull Oxidation Step Oxidation of the skull in an argonoxygen atmosphere at $750^{\circ} \mathrm{C}$ will oxidize the actinide, metal and noble metal atoms that were mechanically retained in the dross to actinide, general and noble metal oxides. The entire skull can subsequently be converted to a powder. This powder then is submitted to the skull reclamation step [44]. 
4.4.3.2.2 Skull Reclamation Step The powder is suspended in a liquid halide, where the noble metal oxides are reduced to noble metal atoms by adding $Z n$, which has a higher oxidation potential than noble metals. Liquid halide can be considered an electrolyte. Noble metal oxides therefore dissociate in liquid halides and may recombine with electrons to metal atoms. The noble metal atoms then are extracted mechanically. Actinide metal oxides are subsequently reduced to actinide metal atoms by adding $M g Z n$ alloy, which has a higher oxidation potential than $Z n$. This is necessary, because actinides also have a higher oxidation potential than noble metals. The actinide metal atoms then will precipitate and can mechanically be separated from the remainder of the halide. Some actinides, however, will remain in the halide and hence considering radioactive waste from $E B R-I I$ as actinide free is only a good approximation [44].

\subsubsection{IFR Pyroprocess}

\subsubsection{Electro-refining Process}

The electro-refining process basically comprises two steps. First, sections of spent driver and breeder fuel elements are submerged in an electrolyte for dissolution. Subsequently, an applied electric potential difference in the electrolyte effects the separation of actinides and fission and activation products, eventually resulting in the precipitation of the actinides on the cathode and the fission and activation products remaining in the electrolyte $[5]$.

4.4.4.1.1 Dissolution Step The oxidant in the electro-refining process is chlorine. Liquid $\mathrm{LiCl}-\mathrm{KCl}$ eutecticum at $500^{\circ} \mathrm{C}$ is used as the electrolyte. Due to the very high oxidation potential of the alkali metals $L i$ and $K$, the electrolyte will undergo almost no dissociation, i.e. will have almost no metal ions and chlorine ions in it. Addition of $C d C l_{2}$ will release 
a sufficient amount of chlorine ions because of the low oxidation potential of the general metal $C d[5]$.

The spent fuel element sections contain spent fuel, adhering sodium thermal bond and clad material. The latter is stainless steel and due to the effect of passivation it will not be affected, once the spent fuel element sections are submerged into the electrolyte. Depending on their oxidation potentials, most metals present in the spent fuel and the sodium thermal bond will with different intensities form metal chlorides that partly dissociate into metal and chlorine ions subsequently. This represents the dissolution.

High oxidation potential metals will almost entirely form metal chlorides with only a minor share remaining as metal atoms and very few metal chlorides dissociating into ions. Low oxidation potential metals will almost entirely remain as metal atoms with only a minor share forming metal chlorides and very many metal chlorides dissociating into ions.

Supplying an external energy source in the form of a an electric potential will enhance this dissolution process. The metal atoms to be dissolved have to form the anode so that chlorine ions will be attracted to the site of oxidation. Also, the metal ions that are a result of dissociation after metal chloride formation will be repelled from the site of oxidation, both effects together enhancing the reaction because of the greater distance from equilibrium. The metal ions attracted by the cathode will be reduced to form metal atoms, whereby the cathode will not be oxidized by the metal ions to be reduced only if their elements have a higher oxidation potential than the cathode element and hence can not take electrons from the cathode metal atoms but must receive it from electric current. This is one reason for the choice of liquid cadmium as the material for the cathode. The enhanced dissolution process is referred to as anodic dissolution.

Since the metal chlorides of the elements with a high oxidation potential are produced in large amounts but do not dissociate into many ions and since the metal chlorides of elements with a low oxidation potential that 
do dissociate into many ions are produced in only small amounts, the total number of ions is not very significant to the process. Moreover, after reaching equilibrium, most chlorine ions will be bound in metal chlorides, most metal ions will have been reduced to metal atoms.

Clearly, the noble metals and most of the general metals will remain in the metal atom form, while almost all alkali metals and alkaline earth metals form metal chlorides. To a lesser extent this holds true for the rare earth metals and the actinides, leaving the former ones mostly in their metal chloride form and the latter ones mostly in their metal atom form. Since the general metals and noble metals are relatively inert as metal atoms and the alkali and alkaline earth metals as metal chlorides, due to their low and high oxidation potential, respectively, only the metal chlorides of the rare earths (lanthanides) and the metal atoms of the actinides will be affected by the following separation step.

4.4.4.1.2 Separation Step Imposing a potential difference on the electrolyte after the dissolution or anodic dissolution step represents the separation step. At the anode, oxidation of actinide metal atoms to metal ions occurs and at the cathode, lanthanide metal chlorides will be reduced to metal ions and chlorine ions. The latter ones combine with the metal ions at the anode to form actinide metal chlorides. Then actinide metal chlorides are reduced at the cathode also. They even control the reduction process after a while, because the oxidation potential of actinides is lower than the one of lanthanides, i.e. the affinity of actinides for reduction is higher. Thus the cathode will have initially a high lanthanide metal ion precipitation rate, but then the actinide metal ion precipitation rate becomes much higher than the one for lanthanides. The cathode will thus contain mostly actinides with some lanthanide impurity. The lanthanides mostly remain in the electrolyte as metal chlorides.

It takes a cathode and anode metal with an oxidation potential lower than the one for actinides and lanthanides. Otherwise the chlorine ions just 
released at the cathode by reduction of the metal chlorides would oxidize the cathode and the metal ions just produced at the anode would reclaim their electrons from the cathode metal and thus oxidize or dissolve it, respectively.

If the cathode metal has an oxidation potential way below the one of actinides, then all actinide metal chlorides are approximately being reduced equally well. If, however, the cathode metal oxidation potential is in the vicinity of the one for actinides, then the actinide metal chloride of the actinide with the nearest oxidation potential will be reduced preferentially, in this case uranium. In general, the minor actinides behave like plutonium and thus accompany it in the various processes.

Hence by chosing two different cathode metals, the goal of precipitating driver and breeder fuel can be achieved in one step, i.e. the lower oxidation potential cathode metal precipitates all actinides and provides the driver fuel, the higher oxidation potential cathode metal precipitates uranium slightly better than the other actinides and hence provides the breeder fuel. Chosing uranium as the higher oxidation potential cathode allows the entire cathode with adhering uranium precipitation to be treated as an entity in the following recovering process. As to the lower oxidation potential cathode, liquid cadmium again lends itself, as well as for the anode [5].

\subsubsection{Purifying Process}

The contamination of the actinides at the cathodes with lanthanides makes necessary the purifying process, which is essentially a retorting or distillation process. Both lower and higher oxidation potential cathode with actinide and uranium precipitation are melted so that the lanthanides evaporate. The uranium deposition on the low oxidation cathode has to be mechanically removed prior to retorting, if a different material than uranium is used for this cathode. The remainder of the retorting process represents highly purified uranium or actinides, ready for refabrication of new fuel [5].

Efficiency of the electro-refining process requires plutonium at the liquid 
cadmium cathode to go in solution beyond saturation, thus intermetallic compounds with $C d$ form and precipitate. Uranium is preferentially deposited at the solid uranium cathode so that it will not eceed its saturation at the liquid cadmium cathode and will not form intermetallic compounds with $C d$. However, intermetallic compounds are expected to decompose in the retorting process [5].

\subsubsection{Extracting and Stripping Process}

Noble and general metals contaminate the liquid cadmium anode after a while, which renders necessary a mechanical purification of this cadmium. Alkali metal, alkaline earth metal and rare earth metal chlorides contaminate the $\mathrm{LiCl}-\mathrm{KCl}$ electrolyte after a while. Adding a $\mathrm{Li}-\mathrm{K}-\mathrm{Cd}$ alloy reduces most of the alkaline and rare earth metal chlorides to metal atoms or initiates formation of intermetallic compounds with $C d$, which can be removed mechanically. Thus the electrolyte will be purified, too. Cesium, strontium and iodine, however, remain in the electrolyte as chlorides due to their very high oxidation potential. The accumulation of these makes further use of the electrolyte impossible after a while [5].

In addiditon, the electrolyte contains traces of actinides that were not deposited on the cathodes. The same holds true for the cadmium that serves as the cathode during the dissolution step and as the anode during the separation step. The actinide content can be as high as $1 \%$ of the initial actinide charge to the process. Also, the incomplete dissolution step leaves traces of actinides (and fission products) at the stainless steel clads [5].

In order for the electrolyte, the cadmium and the clads to be considered as Non-Transuranic waste (NTRUW), all those trace contaminations have to be reduced from the said $1 \%$ or $10^{-2}$ to $10^{-6}$. An attempt to achieve this is done with the extracting of non-uranium actinides in exchange for uranium and the subsequent removal or stripping of uranium.

In the extracting process, the electrolyte is brought into contact with a 
cadmium-uranium alloy. Although uranium has a slightly lower oxidation potential than the remainder of the actinides, and hence would not be affected by the non-uranic actinides in the electrolytes, the presence of $C d$ serves the same purpose as in the case of actinide precipitation on the higher oxidation potential cathode in the separation step of the electrorefinig process. Thereby part of the uranium in the $C d-U$ alloy will dissolve into the electrolyte in exchange for non-uranic actinides, which are reduced and deposited at the cadmium-uranium alloy in order to reach equilibrium in the electrolyte. If the uranium fraction in this alloy is high enough almost all the non-uranic actinides can be extracted. The then cadmium-actinide alloy can be retorted in order to separate cadmium for reuse and actinides for refabrication of driver fuel [5].

The stripping process removes the uranium, which now has a higher content than before the extracting process, from the electrolyte by adding a cadmium-lithium alloy. Again, the presence of cadmium in $C d-L i$ has a stabilizing effect on actinides and thus the lithium will be dissolved in exchange for uranium (and very few non-uranic actinides). The cadmiumuranium alloy together with the stainless steel clads and the cadmium will be retorted in order to recover any cadmium. The remainder of this retorting process contains general and noble metals, very few uranium and even less non-uranic actinides; it is considered as the first of two significant waste streams. The electrolyte contains alkali and alkaline earth metals with strontium, cesium and iodine as the dominant fission products as well as a vanishing amount of actinides and is considered the second significant waste stream [5].

The actinide content in both waste streams will correspond to less than $10^{-4}$ of the initial actinide charge to the process. Further investigation and implementation of an additional process will bring this number down to the $10^{-6}$ required for the waste streams to be considered as NTRUW [14]. Rare earth metals or lanthanides are distributed between both waste streams, 
with much less lanthanides in the electrolyte waste than in the retorting waste. Consequently, as of this time, considering radioactive waste from $I F R$ as actinide free is only a good approximation. 


\section{Chapter 5}

\section{Comparison of the Reactors}

\subsection{Introduction}

To understand the essence of the comparison conducted in this study, it is important to devote some attention to the objects that are being compared, i.e. the reactor components and the reactors themselves. Also of significance is the way the comparison is presented.

\subsubsection{Reactor Components as Radioactive Waste}

The radioactive waste parameters defined in Chapter 2 serve to characterize the radioactive waste produced by both fast fission and fusion reactors. A comparison of fast fission and fusion reactors oriented to these radioactive waste parameters, however, must specify to which components of the reactors they apply. In this study, distinct functions within the reactor define a component as outlined in Chapter 4. Typical components can be found for fast fission reactors and for fusion reactors, allowing comparison among them.

For experimental and commercial fast fission reactors the following components can be defined by their functions. These components allow comparison among fast fission reactors.

1. Fuel (Actinides) 
2. Fuel (Fission Products and Activation Products)

3. Fuel (Actinides and Fission Products and Activation Products)

4. Clad

5. Duct

6. Reflector

7. Shield

For experimental and commercial fusion reactors the following components can be defined by functions. These components allow comparison among fusion reactors.

1. Protective Layer

2. First Wall

3. Breeder Blanket

4. Back Wall or Manifold

5. Shield

6. Vacuum Vessel

7. Toroidal Coils

8. Poloidal Coil

9. Cryostat Coolant

10. Cryostat Vessel

As noted in Chapter 4, a fast fission reactor can be separated into a driver, internal breeder, radial breeder, reflector and shield section, a fusion reactor can be separated into an inboard and outboard section. Each fast fission and fusion reactor section contains one or more of the above 
components. The separation into sections allows for a more detailed characterization of a reactor itself, but is not very important with respect to the comparison envisioned in this study. Hence, although the separation into sections has been made for the calculation, it is eliminated for the comparison by combining corresponding components of the sections.

A comparison between fast fission and fusion reactors can be conducted, if corresponding components from fast fission and fusion reactors can be found. However, even if there is no exact correspondence between fast fission and fusion reactor components, a comparison can still list the components in the order of highest radioactive waste parameters. The components considered for the comparison and the reasons for their choice are briefly listed here.

The function of the fission breeder fuel is to breed from ${ }^{238} U$ the ${ }^{239} \mathrm{Pu}$ and ${ }^{241} \mathrm{Pu}$ which is necessary to maintain a self-sufficient fuel cycle. Besides the fact that some breeding also happens in the driver fuel, both driver and breeder fuel represent the major heat producing component of a fast fission reactor. The function of the fusion breeder blanket is to breed from ${ }^{6} \mathrm{Li}$ and ${ }^{7} \mathrm{Li}$ the ${ }^{3} \mathrm{H}$ which is necessary to maintain a self-sufficient fuel cycle. It is also the major heat producing component of a fusion reactor. It therefore appears to be reasonable to match the fuel component to the breeder blanket component, the more since both are neutron producing and absorbing components.

The clad in fast fission reactors separates the fuel from the remainder of the reactor and the first wall (and the back wall or the manifold) does fulfill the same function for the breeder blanket in fusion reactors. Clad and first wall are exposed to the highest neutron flux, both from inside and outside the fuel and the breeder blanket. It thus is obvious to match the clad component to the first wall component.

The duct in fast fission reactors has the function of guiding the coolant flow, comparable to the manifold (and the back wall) in fusion reactors. Also, the duct in fast fission reactors is one of the components with a higher 
neutron flux, as is the manifold (and the back wall) in fusion reactors. Hence it is appropriate to match the duct component to the manifold component and/or the back wall component.

Finally, it is straight forward to match the shields, since their function is unambiguous. Except for those components (clad/first wall, fuel/breeder blanket, duct/manifold/back wall, shield/shield), no other components will be considered for the comparison.

Most actinides considerably affect the radioactive waste characterization at any time after discharge from the reactor. In particular the difficulty of radioactive waste handling immediately after discharge is being underestimated if actinides are completely ignored. However, a comparison between fuel with and without actinides can give an idea of how serious this underestimation is. Only the specifc radioactive waste parameters need to be compared, since both actinides and fission products and activation products are contained in the same fuel volume for a given reactor.

As examples, Figures 5.1, 5.2 and 5.3 compare the specific radioactivity, the specific deacy power and the specific Biological Hazard Potential of the actinides to those of the fission and activation products. Until about $100 y$ after discharge, the above parameters for the actinides are equal to or less than those for the fission and activation products. Thereafter the parameters for the actinides are about three orders of magnitude greater than those for the fission and activation products. However, the parameters for $0.1 \%$ of the actinides and those for the fission and activation products would have the same order of magnitude even for times greater than $100 y$ after discharge.

Reprocessing for both $E B R-I I$ and $I F R$ takes place within a few years after discharge, so that actinides in the radioactive waste can indeed be ignored for that time. Even if $0.1 \%$ of the actinides would remain in the radioactive waste, their contribution would then indeed be negligible.

Note, that all three radioactive waste parameters of the actinides from 


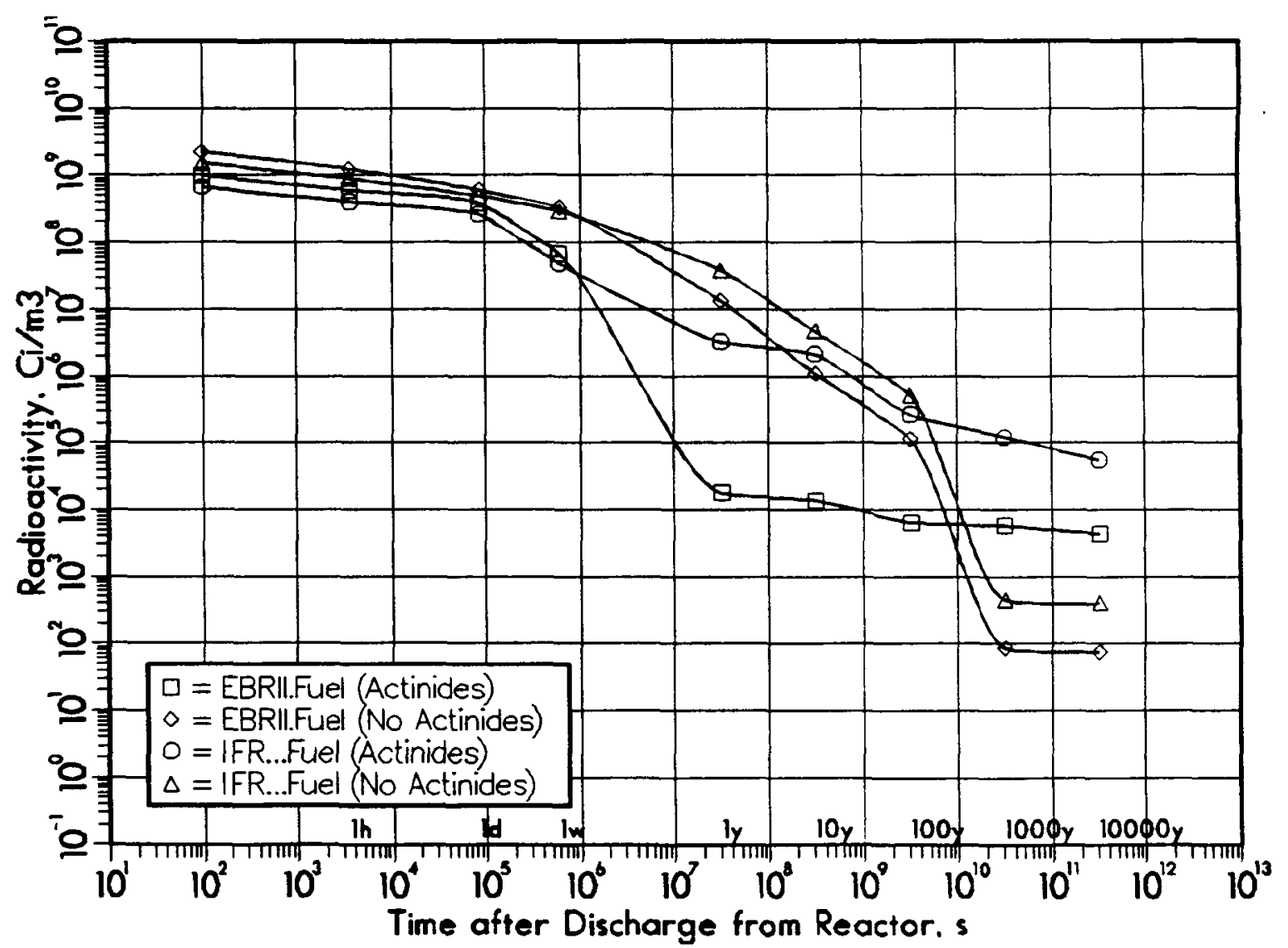

Figure 5.1: Comparison of Specific Radioactivity of Fission Products, Activation Products and Actinides, EBRII and IFR 


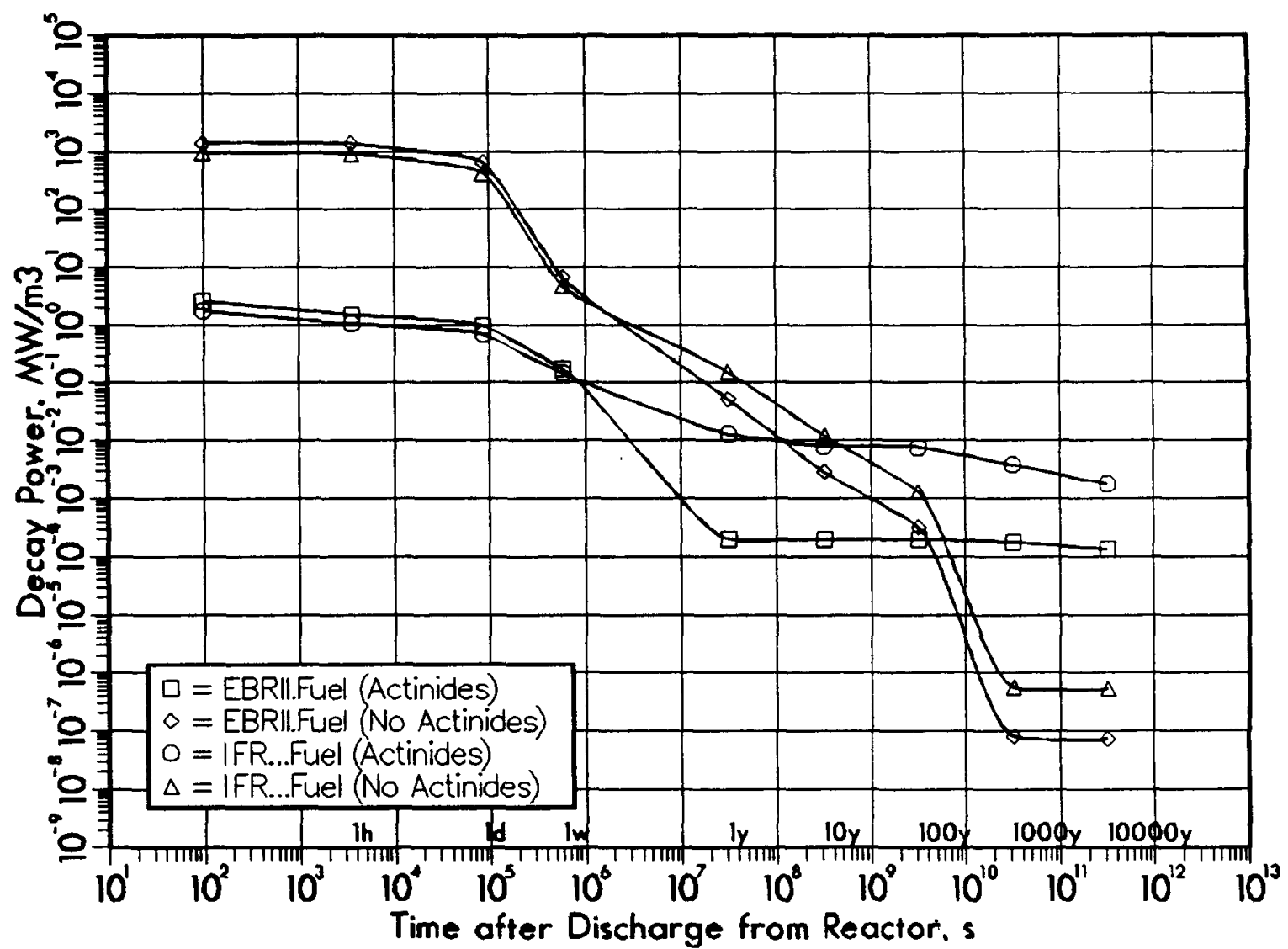

Figure 5.2: Comparison of Specific Decay Power of Fission Products, Activation Products and Actinides, EBRII and IFR 


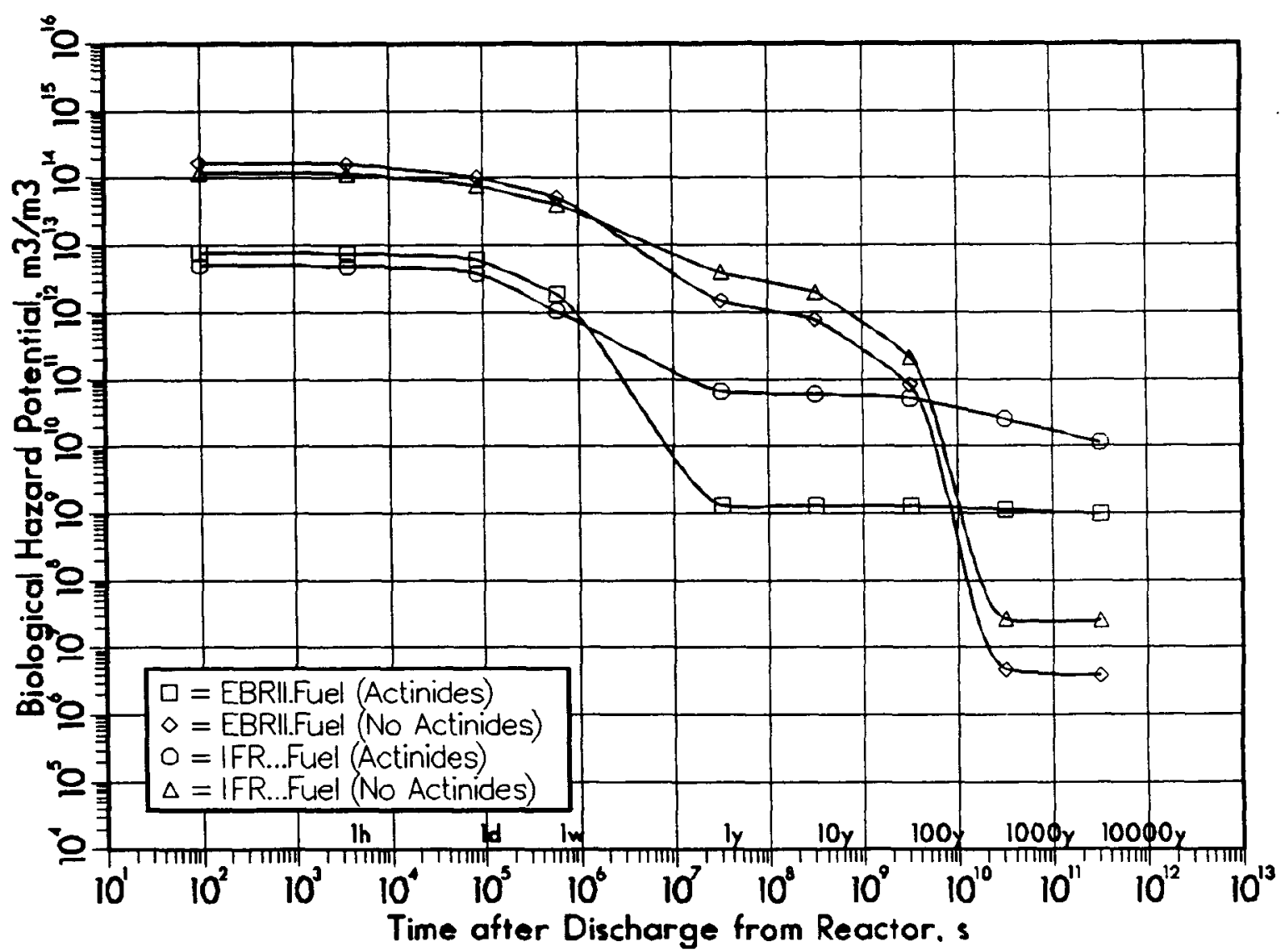

Figure 5.3: Comparison of Specific Biological Hazard Potential of Fission Products, Activation Products and Actinides, EBRII and IFR 
$E B R-I I$ drop drastically within one year after discharge, while the ones of the actinides from IFR remain at a relatively high level. A detailed analysis of the actinide nuclides shows that within one year after discharge the radioactive waste parameters are determined by plutonium. The plutonium isotopes ${ }^{239} \mathrm{Pu}$ and ${ }^{241} \mathrm{Pu}$ are dominant. They are produced by transmutation of the uranium isotope ${ }^{238} U$. As explained in Section 4.2, due to its high enrichment in ${ }^{235} U$, EBR-II fuel contains less ${ }^{238} U$ than $I F R$ fuel. Also, $E B R$-II fuel contains almost no plutonium, while one fifth by weight of the $I F R$ fuel is plutonium. Adding to those facts the low burnup of $E B R-I I$ fuel yields the explanation for the drastic drop of its actinide radioactive waste parameters within one year after discharge. The displayed behavior of the three radioactive waste parameters of the $E B R-I I$ actinides is typical of highly ${ }^{235} U$ enriched fuel.

\subsubsection{Cycle, Life-time \& Decommissioning Radioac- tive Waste}

Comparing four rather different reactors with respect to radioactive waste does not seem fair, since due to different operation schedules as outlined in Chapter 4, all of them will have different neutron fluences at the time a component is considered radioactive waste. However, this study is not a mere comparison of isolated components under irradiation, where a constant fluence is important to ensure comparability of isolated components, but rather a comparison of components in different reactor systems. In particular, different operational requirements of fusion and fast fission reactors are accounted for and hence different neutron fluences for these reactors are inherent to the topic of this study. Also, a comparison of radioactive waste, i.e. components on a "per year" basis would distort the effect of operational requirements.

Operation schedules for experimental and commercial reactors are fundamentally different. In experimental reactors, the duration of irradiation 
is determined by the requirements of the experiments being conducted, which rarely follow a periodic plan or cycle. The intensity of irradiation may also vary from one experiment to the next. In commercial reactors on the other hand, the duration of irradiation is ruled by efficiency, which is best achieved in following a periodic plan or cycle. Also, the intensity of irradiation is almost constant over time.

For these reasons, radioactive waste from experimental reactors shall be assessed for the entire life-time of the reactor. In the case of $E B R-I I$, the lifetime is assumed to be $4 y$. The 500 driver and internal breeder assemblies, the 100 radial breeder assemblies and the 162 shield assemblies discharged during the life-time are irradiated for $135 d$ each and $4 y$, respectively. In the case of ITER, the life-time is $12.5 y$. The components are irradiated for these $12.5 y$.

Radioactive waste from commercial reactors can be assessed for a cycle. However, there are some components in commercial reactors that will be irradiated for the entire life-time of the reactor as well and thus will not follow a cycle at all. While the former ones shall be referred to as cycled components, the latter ones shall be designated non-cycled components. In the case of $I F R$, a cycle is $1 y$ and the cycled components correspond to $25 \%$ of the core. The cycled components are irradiated for $4 y$. In the case of RAFHT, a cycle is $6 y$ and the cycled components correspond to $100 \%$ of the first wall, breeder blanket and manifold. The cycled components are irradiated for $6 y$. The non-cycled component is equivalent to $100 \%$ of the shield for both IFR and RAFHT, which have a life-time of $30 y$.

Under this prerequisite, the operation schedules given in Chapter 4 can be summarized in terms of the duration of irradiation and the components that are irradiated for this duration, as given in Table 5.1.

Specific radioactive waste parameters only depend on the neutron fluence received during irradiation and can therefore be compared easily for corresponding components. Absolute radioactive waste parameters heavily 


\begin{tabular}{|l|r|r|}
\hline \hline Reactor & Component & $\begin{array}{r}\text { Duration of } \\
\text { Irradiation }\end{array}$ \\
\hline Experimental Fission & $\frac{1}{500}$ Clad $/ \frac{1}{100}$ Clad & 135 days \\
\cline { 2 - 3 } Reactor: EBR-II & $\frac{1}{50}$ Fuel $\frac{1}{10}$ Fuel & 135 days \\
\cline { 2 - 3 } & $\frac{1}{500}$ Duct $/ \frac{1}{100}$ Duct & 135 days \\
\cline { 2 - 3 } & Steel Shield & 4 years \\
\hline Commercial Fission & $\frac{1}{4}$ Clad & 4 years \\
\cline { 2 - 3 } Reactor: IFR & $\frac{1}{4}$ Fuel & 4 years \\
\cline { 2 - 3 } & $\frac{1}{4}$ Duct & 4 years \\
\cline { 2 - 3 } & Steel Shield & 30 years \\
\hline Experimental Fusion & First Wall & 12.5 years \\
\cline { 2 - 3 } Reactor: ITER & Breeder Blanket & 12.5 years \\
\cline { 2 - 3 } & Back Wall & 12.5 years \\
\cline { 2 - 3 } & Steel Shield & 12.5 years \\
\hline Commercial Fusion & First Wall & 6 years \\
\cline { 2 - 3 } Reactor: RAFHT & Breeder Blanket & 6 years \\
\cline { 2 - 3 } & Manifold & 6 years \\
\cline { 2 - 3 } & Steel Shield & 30 years \\
\hline \hline
\end{tabular}

Table 5.1: Summary of Operation Schedules 
depend on the presence or absence of cycles and therefore a comparison of radioactive waste parameters for corresponding components is not quite as easy.

To determine life-time absolute radioactive waste parameters for commercial reactors, it is necessary to multiply absolute radioactive waste parameters of cycled components by the number of cycles per life-time. Absolute radioactive waste parameters of non-cycled components do not have to be modified.

This procedure represents an idealization of the reality for commercial reactors, because irradiated components of different cycles amount with a time distance of a cycle's duration, during which some decay of activation is allowed to take place. So the actual life-time radioactive waste will have lower radioactive waste parameters than the idealized life-time radioactive waste. However, it could be argued that a number of commercial reactors equal to the number of cycles per life-time of a reactor exists and the idealized life-time radioactive waste would then be reactor compound radioactive waste.

Another important concept is the radioactive waste present in the reactor at the time of decommissioning. For experimental reactors, decommissioning radioactive waste is equal to life-time radioactive waste, assuming that the experimental reactors will be decommissioned after the experiment has been conducted. For commercial reactors, decommissioning radioactive waste represents the cycled components of the final cycle, expanded by the non-cycled components. Decommissioning is assumed to take place $10 y$ after reactor shut down or end of life-time, which is equivalent in this study to $10 y$ after discharge of the components from the reactor.

Definitions of cycle, life-time and decommissioning radioactive waste in this study are displayed in Table 5.2 for the different reactors. 


\begin{tabular}{|l|r|r|r|}
\hline \hline Reactor & $\begin{array}{r}\text { Cycle } \\
\text { Radioactive } \\
\text { Waste (EOC) }\end{array}$ & $\begin{array}{r}\text { Life-time } \\
\text { Radioactive } \\
\text { Waste (EOL) }\end{array}$ & $\begin{array}{r}\text { Decommissioning } \\
\text { Radioactive } \\
\text { Waste }(\text { DEC) }\end{array}$ \\
\hline \multirow{4}{*}{ EBR-II } & - & $500 \times \frac{1}{500} / 100 \times \frac{1}{100}$ Clad & $500 \times \frac{1}{50} / 100 \times \frac{1}{100}$ Clad \\
\cline { 2 - 4 } & - & $500 \times \frac{1}{500} / 100 \times \frac{1}{100}$ Fuel & $500 \times \frac{1}{500} / 100 \times \frac{1}{100}$ Fuel \\
\cline { 2 - 4 } & - & $500 \times \frac{1}{500} / 100 \times \frac{1}{100}$ Duct & $500 \times \frac{1}{500} / 100 \times \frac{1}{100}$ Duct \\
\cline { 2 - 4 } & - & $1 \times$ Steel Shield & $1 \times$ Steel Shield \\
\hline \multirow{4}{*}{ IFR } & $1 \times \frac{1}{4}$ Clad & $30 \times \frac{1}{4}$ Clad & $4 \times \frac{1}{4}$ Clad \\
\cline { 2 - 4 } & $1 \times \frac{1}{4}$ Fuel & $30 \times \frac{1}{4}$ Fuel & $4 \times \frac{1}{4}$ Fuel \\
\cline { 2 - 4 } & $1 \times \frac{1}{4}$ Duct & $30 \times \frac{1}{4}$ Duct & $4 \times \frac{1}{4}$ Duct \\
\cline { 2 - 4 } & - & $1 \times$ Steel Shield & $1 \times$ Steel Shield \\
\cline { 2 - 5 } & - & $1 \times$ First Wall & $1 \times$ First Wall \\
\cline { 2 - 5 } & - & $1 \times$ Breeder Blanket & $1 \times$ Breeder Blanket \\
\cline { 2 - 5 } & - & $1 \times$ Back Wall & $1 \times$ Back Wall \\
\cline { 2 - 5 } & - & $1 \times$ Steel Shield & $1 \times$ Steel Shield \\
\cline { 2 - 5 } & $1 \times$ First Wall & $5 \times$ First Wall & $1 \times$ First Wall \\
\cline { 2 - 5 } & $1 \times$ Breeder Blanket & $5 \times$ Breeder Blanket & $1 \times$ Breeder Blanket \\
\cline { 2 - 5 } & $1 \times$ Back Wall & $5 \times$ Back Wall & $1 \times$ Back Wall \\
\cline { 2 - 5 } & & $1 \times$ Steel Shield & $1 \times$ Steel Shield \\
\hline \hline
\end{tabular}

Table 5.2: Definition of Cycle, Life-time and Decommissioning Radioactive Waste 


\subsubsection{Presentation of the Comparison of Radioactive Waste}

The comparison will be conducted in separate sections for each radioactive waste parameter. Each of those sections, with the exception of the one on volumes, will contain figures showing specific and/or life-time absolute radioactive waste parameters of the afore mentioned components. Experimental and commercial reactors are presented in separate figures.

Figures only have a limited capability of resolution, so it is not possible to display experimental and commercial reactors in the same figure and the restriction to a reasonable number of figures prevents displaying figures comparing fusion and fission reactors separately in addition to figures comparing experimental and commercial reactors separately.

To make up for the possible loss in overview, tables are supplied in Appendix B that list specific radioactive waste parameters as well as cycle, life-time and decommissioning absolute radioactive waste parameters for all afore mentioned components of all reactors. Each table covers a certain time after discharge. However, to keep the number of tables reasonably low, only three times were considered. Those are 10y, 100y and 1,000y after discharge of the components from the reactor. These three points in time represent the short-term, intermediate-term and long-term characterization of radioactive waste.

Appendix B will also contain figures showing specific and life-time absolute radioactivity and decay power for the sum of the above components of each reactor. This allows a ranking of the reactors with respect to their overall radioactive waste behavior.

A short section will be devoted to the radionuclides that determine most radioactive waste parameters.

As detailed in Chapter 4, each of the reactors under consideration has a different nominal thermal and electrical power output. IFR is a $350 M W_{e}$ reactor, while $R A F H T$ generates $1,200 M W_{e}$. Also, EBR-II is rated at 
$60 M W_{t h}$, while ITER is rated at $1,000 M W_{t} h$. The radioactive waste parameters are not normalized to the power output, but are evaluated and presented for the reactors as built. Therefore, the different power output should always be kept in mind when looking at the comparison.

\subsection{Volume}

The biggest disadvantage of fusion reactors is the volume of radioactive waste produced. Table 5.3 lists those volumes of the different reactor components and reactors for cycle, life time and decommissioning radioactive waste.

The energy per nucleus and neutron released in the fusion reaction is greater than the one released in the fission reaction. However, the energy released in a fission reaction is mostly transferred to fission products which have a high heat generating cross section with nuclides of a high concentration material - the fuel. The energy released in a fusion reaction is mostly transferred to neutrons which have a relatively low heat generating cross section with nuclides of a low concentration material - the breeder blanket. The only way to compensate for low heat generating cross sections and low concentrations is to provide a sufficiently large volume of the respective material.

Thus, as far as the fuel component and breeder blanket component are concerned, larger volumes for fusion reactors as opposed to fast fission reactors are inherent to the indirect way the fusion reaction is utilized in tokamaks. The energy density of a fast fission reactor is simply much higher than that of a fusion reactor and the volumes of fuel and breeder blanket component differ by about three orders of magnitude.

Although the high energy density of a fast fission reactor is beneficial, it requires dividing up the fuel component into thousands of small units in order to allow removal of the generated heat. Each such unit must be contained by a clad unit, while the breeder blanket component of fusion reactors may be contained by just one large first wall component. The 


\begin{tabular}{|c|c|c|c|c|c|}
\hline \multirow{2}{*}{\multicolumn{2}{|c|}{ Para }} & \multicolumn{2}{|c|}{ Fission Reactors } & \multicolumn{2}{|c|}{ Fusion Reactors } \\
\hline & & EBR-II & IFR & ITER & RAFH' \\
\hline \multirow{4}{*}{$\begin{array}{l}\text { EOC-Volume } \\
\text { EOL-Volume } \\
\text { DEC-Volume }\end{array}$} & \multirow[b]{2}{*}[m^{3}]{} & \multicolumn{2}{|c|}{ Clad } & \multicolumn{2}{|c|}{ First Wall } \\
\hline & & \multicolumn{2}{|l|}{ = } & - & \\
\hline & & $0^{-1}$ & & $10^{+0}$ & \\
\hline & & & & $\cdot 10^{+0}$ & $10^{+0}$ \\
\hline \multirow{4}{*}{$\begin{array}{l}\text { EOC-Volume } \\
\text { EOL-Volume }\end{array}$} & \multirow{4}{*}{$\begin{array}{l}{\left[m^{3}\right]} \\
{\left[m^{3}\right]} \\
{\left[m^{3}\right]}\end{array}$} & \multicolumn{2}{|c|}{ Fuel } & \multicolumn{2}{|c|}{ Breeder Blanket } \\
\hline & & - & $4.3 \cdot 10^{-1}$ & - & $3.9 \cdot 10^{+2}$ \\
\hline & & $.0 \cdot 10^{-1}$ & & $.3 \cdot 10^{+2}$ & $10^{+3}$ \\
\hline & & & & $2.3 \cdot 10^{+2}$ & $3.9 \cdot 10^{+2}$ \\
\hline \multirow{4}{*}{\multicolumn{2}{|c|}{$\begin{array}{l}\text { EOC-Volume } \\
\text { EOL-Volume } \\
\text { DEC-Volume }\end{array}$}} & \multicolumn{2}{|c|}{ Duct } & \multicolumn{2}{|c|}{ Back Wall/Manifold } \\
\hline & & $=$ & $10^{-1}$ & $=$ & $3.4 \cdot 10^{+1}$ \\
\hline & & $8 \cdot 10^{-1}$ & & $4.3 \cdot 10^{+1}$ & $10^{+2}$ \\
\hline & & $8 \cdot 10^{-1}$ & $7.6 \cdot 10^{-1}$ & $.3 \cdot 10^{+1}$ & $3.4 \cdot 10^{+1}$ \\
\hline \multirow{4}{*}{\multicolumn{2}{|c|}{$\begin{array}{l}\text { EOC-Volume } \\
\text { EOL-Volume } \\
\text { DEC-Volume }\end{array}$}} & \multicolumn{2}{|c|}{ Steel Shield } & \multicolumn{2}{|c|}{ Steel Shield } \\
\hline & & - & & & \\
\hline & & +0 & +0 & $1 \cdot 10^{+2}$ & $10^{+2}$ \\
\hline & & $0^{+0}$ & $6.4 \cdot$ & $\cdot 10^{+2}$ & $10^{+}$ \\
\hline \multirow{4}{*}{$\begin{array}{l}\text { EOC-Volume } \\
\text { EOL-Volume }\end{array}$} & \multirow{4}{*}{$\begin{array}{l}{\left[\mathrm{m}^{3}\right]} \\
{\left[\mathrm{m}^{3}\right]}\end{array}$} & \multicolumn{2}{|c|}{ Reactor } & \multicolumn{2}{|c|}{$\mathbf{R e}$} \\
\hline & & - & 9.3. & - & $4.3 \cdot 10^{+2}$ \\
\hline & & & & & $10^{+3}$ \\
\hline & & +0 & $1.0 \cdot 10^{-7}$ & $6.0 \cdot 10^{+2}$ & $6.1 \cdot 10^{+2}$ \\
\hline
\end{tabular}

Table 5.3: Volumes of Cycle, Life Time and Decommissioning Radioactive Waste 
large number of clad units generates a clad component that is comparable in volume to the first wall component.

The commercial fusion reactor can be run with significantly less cycles than the commercial fast fission reactor. However, because for each cycle the volume of the components of the fusion reactor is so much larger than the one of the components of the fast fission reactor, this fact does not result in a life time volume for fusion reactors less than that for fast fission reactors.

Due to the difference in energy densities of the reactors, the steel shield can also be significantly smaller for fast fission reactors than for fusion reactors.

A comparison of the volumes of experimental and commercial reactor components on the fast fission reactor and fusion reactor side shows, that the ratios of the volumes of components of the experimental and commercial reactor are usually lower for the fast fission reactor than for the fusion reactor.

\subsection{Radioactivity}

The course of the specific radioactivity over time after discharge is shown in Figures 5.4 and 5.5. Although the EBR-II fuel starts out at a specific radioactivity 10 times higher than the one of the ITER first wall right after discharge, it ends up 100 times lower than this at 10,000y after discharge. The fuel then reaches the specific radioactivity of the remainder of the $E B R$ $I I$ and ITER components, which in general is 100 to 1,000 times lower than the one of the first wall at all times after discharge.

The specific radioactivity of those components is spread by a factor of about 10 , with the one of the $E B R-I I$ components tending to be higher than the one of the ITER components right after discharge, but lower than this at 10,000y after discharge. At less than 1,000y after discharge, only the ITER first wall has a specific radioactivity of greater than $1,000 \frac{\mathrm{Ci}}{\mathrm{m}^{3}}$. 
$I F R$ and $R A F H T$ are separated more clearly. The RAFHT first wall starts out at a specific radioactivity 10 times lower than the one of the IFR fuel and also ends up like this at 10,000y after discharge. However, right after discharge, it has the second highest specific radioactivity, while at $10,000 y$ after discharge the specific radioactivity is lower than the one of all IFR components.

The remainder of the $R A F H T$ components has a specific radioactivity that is on the average 10 to 100 times lower than the one of the remainder of the IFR components. While all IFR components show a spread in the specific radioactivity by a factor of about 10 at $10,000 y$ after discharge, the one of the RAFHT components is spread by a factor of 10,000 by then. However, at 1,000y after discharge, all components have a specific radioactivity less than $1,000 \frac{\mathrm{Ci}}{\mathrm{m}^{3}}$ and at $100 y$ after discharge, only the one of the IFR fuel exceeds this figure.

With the specific radioactivity of $E B R-I I$ components only slightly lower than the one of IFR components, the effect of utilization of low activation material in RAFHT as opposed to standard material in ITER becomes obvious. The first wall of ITER and RAFHT is almost equal in specific radioactivity right after discharge, but at $100 y$ after discharge already, the $R A F H T$ first wall specific radioactivity is a factor of 100 lower than the one of the ITER first wall; this ratio is also present at 10,000y after discharge. A similiar behavior can be recorded for the remainder of the RAFHT and ITER components.

This fact can be appreciated even more, if it is taken into account, that the intensity of irradiation in terms of the neutron first wall load is on the average three to six times lower in ITER than in RAFHT. At the same time, the duration of irradiation is only two times longer. The advantage of $R A F H T$ over ITER is bought by a slightly higher specific radioactivity of the RAFHT first wall than the one of the ITER first wall for only the first $10 y$ after discharge. 
The slightly higher specific radioactivity of $I F R$ fuel as opposed to $E B R$ $I I$ fuel in the long-term is partly due to dominant ${ }^{93} \mathrm{Zr}$ in $I F R$ fuel activation products as opposed to dominant ${ }^{93} M O$ in $E B R-I I$ activation products.

The course of the absolute radioactivity over time after discharge is shown in Figures 5.6 and 5.7. Again, the EBR-II fuel starts out with the highest absolute radioactivity, but at less than $1 y$ after discharge, all ITER components display a higher absolute radioactivity than all components of $E B R$-II. At 1,000y after discharge, the EBR-II fuel has reached the absolute radioactivity of the remainder of the $E B R-I I$ components, which in general have an absolute radioactivity less than the one of all ITER components by a factor of 100 to 10,000 . Note, that the absolute radioactivity of all ITER components is within a spread of a factor of 10 , while this spread is on the order of 100 to 1,000 for all EBR-II components. Only the absolute radioactivity of all ITER components is greater than $1,000 \mathrm{C} i$ at $1,000 \mathrm{y}$ after discharge and at $100 y$ after discharge, only the $E B R-I I$ fuel exceeds this figure.

The comparison of the absolute radioactivity of the IFR and RAFHT components shows that it can be appropriate to match the fuel and the breeder blanket component for comparison, since the absolute radioactivity of both is different by less than a factor of 10 and higher than the remainder of the components by a factor of 100 right after discharge and 10 at 10,000y after discharge. The exception is the RAFHT first wall, which shows an absolute radioactivity comparable to the one of the fuel and breeder blanket. At 1,000y after discharge, only the fuel and the breeder blanket have an absolute radioactivity of greater than $1,000 \mathrm{C} i$. The remainder of the components clearly shows a lower absolute radioactivity for RAFHT than for IFR, especially at $10,000 y$ after discharge.

The comparison of the absolute radioactivity highlights the importance of the distinction between specific and absolute radioactive waste parameters, because the slight advantage of $E B R-I I$ over ITER with respect to 


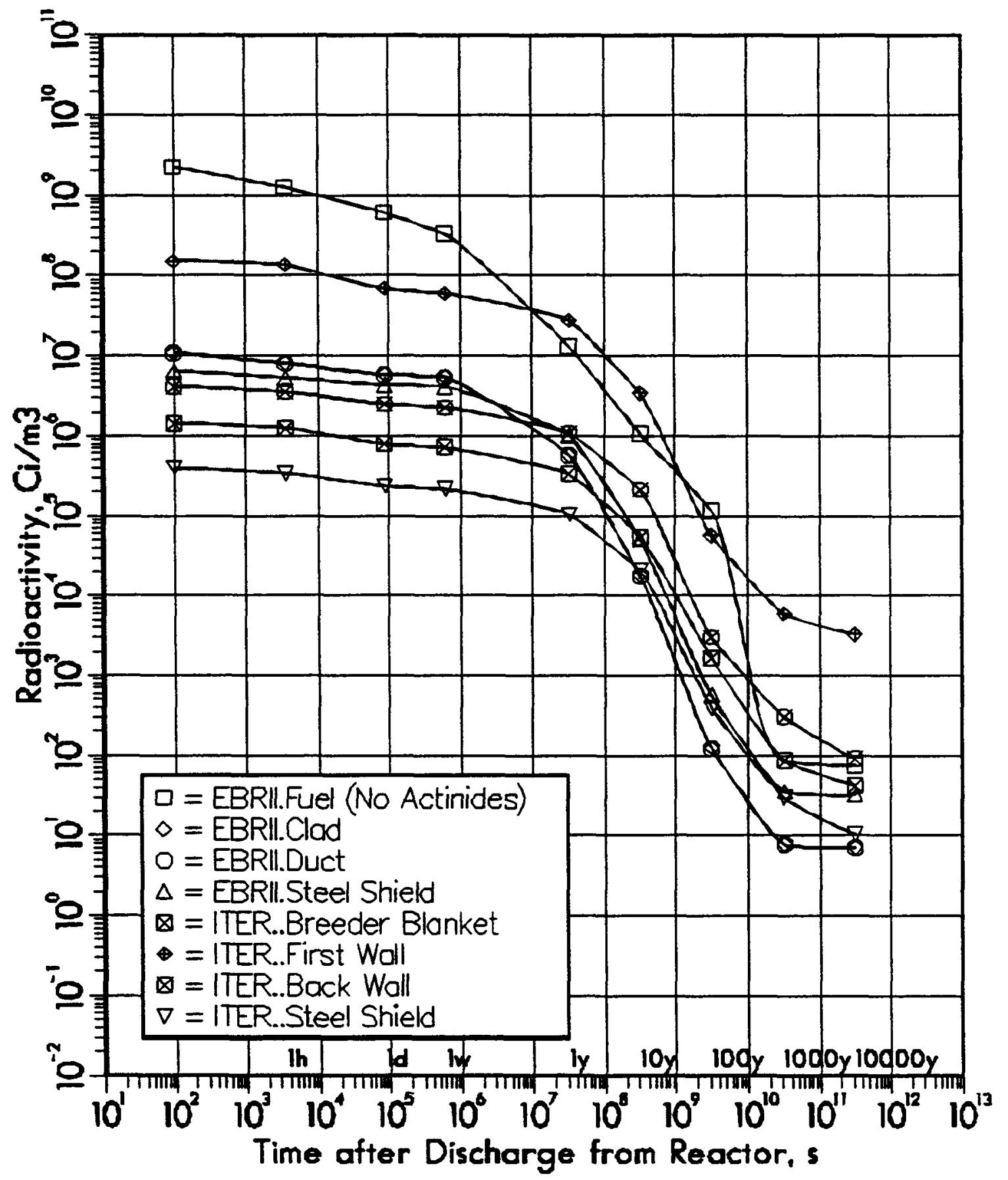

Figure 5.4: Specific Radioactivity EBR-II vs. ITER 


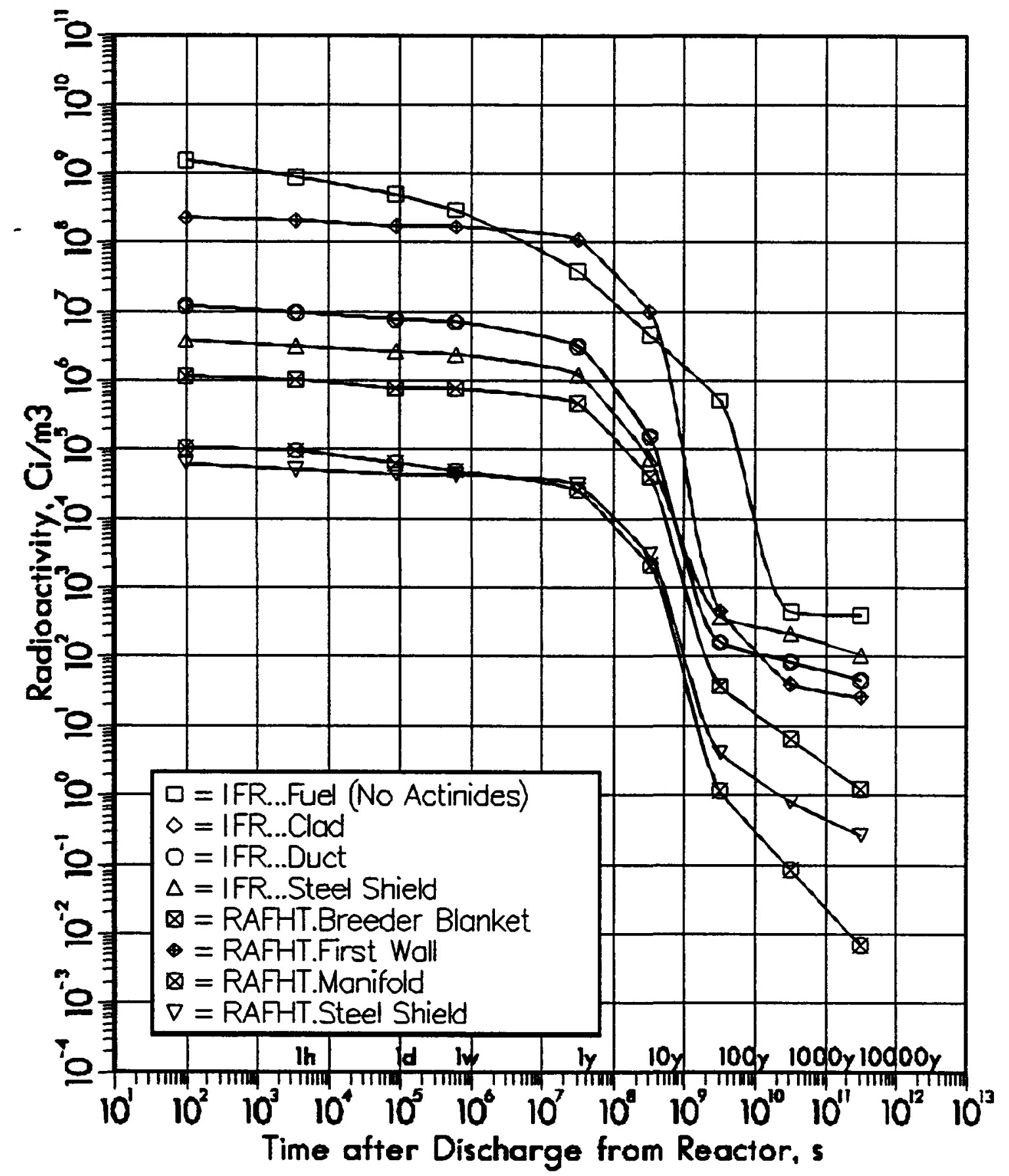

Figure 5.5: Specific Radioactivity IFR vs. RAFHT 
the specific radioactivity turns into a clear advantage with respect to the absolute radioactivity. Also, the advantage of RAFHT over IFR with respect to the specific radioactivity is diminished a little bit with respect to the absolute radioactivity. However, the volumes of RAFHT components are not large enough to turn the specific radioactivity advantage into an absolute radioactivity disadvantage.

Also, the specific radioactivity of ITER components in general is higher than the one of $R A F H T$ components by a factor of 100 to 1,000 , a ratio which is maintained for the absolute radioactivity. And while EBR-II components have approximately the same as or only a slightly lower specific radioactivity than $I F R$ components, the absolute radioactivity of $E B R-I I$ components is generally 10 times lower than the one of IFR components.

It is interesting to note, that the slopes of the graphs in the long-term tend to be zero for fast fission reactor components, but notably negative for fusion reactor components. This could indicate, that the fast fission reactor components either have reached an intermediate plateau or the final equilibrium, where the former case is more likely. Also, the graph for the fuel displays a negative slope that is lower than the one of the graphs for the remainder of the components at the begin of the intermediate term, but higher than this at the end of the intermediate-term. In the short term, the slopes of the graphs for all components are almost zero, with only the one of the graph for the fuel notably negative. The distinct temporal behavior of the fuel is naturally caused by the dominance of its fission products over its activation products. The temporal behavior of the remainder of the components is solely caused by their activation products.

It is worthwhile to mention that Reference [13] cites a value of $6 \frac{\mathrm{Ci}}{\mathrm{m}^{3}}$ for the specific radioactivity of natural uranium. Within $100 y$ after discharge from the reactor, only the shield and the manifold of $R A F H T$ reach this specific radioactivity, and only the breeder blanket of RAFHT can be added to these within 1,000y after discharge. The next closest components are 


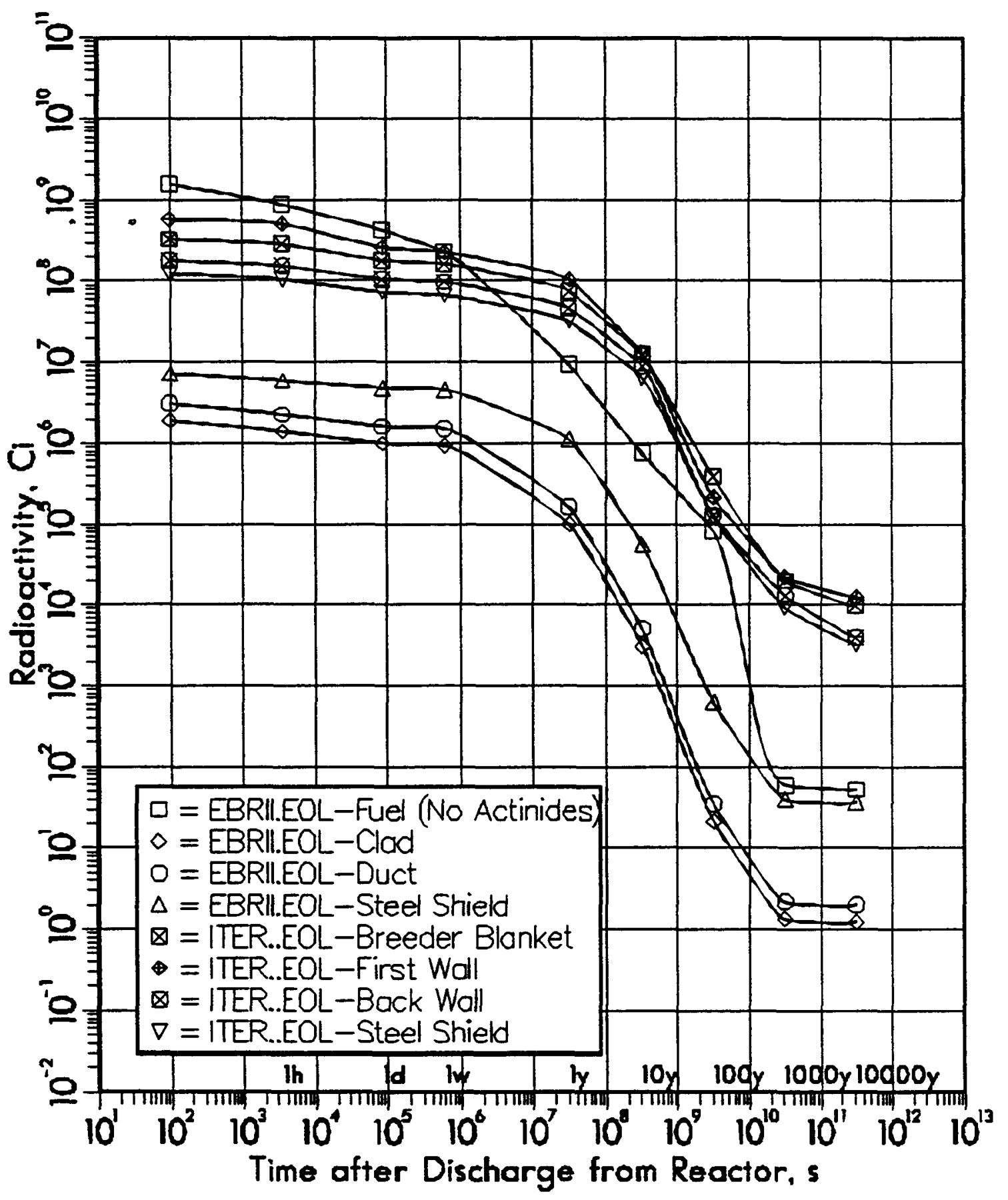

Figure 5.6: Life Time Absolute Radioactivity EBR-II vs. ITER 


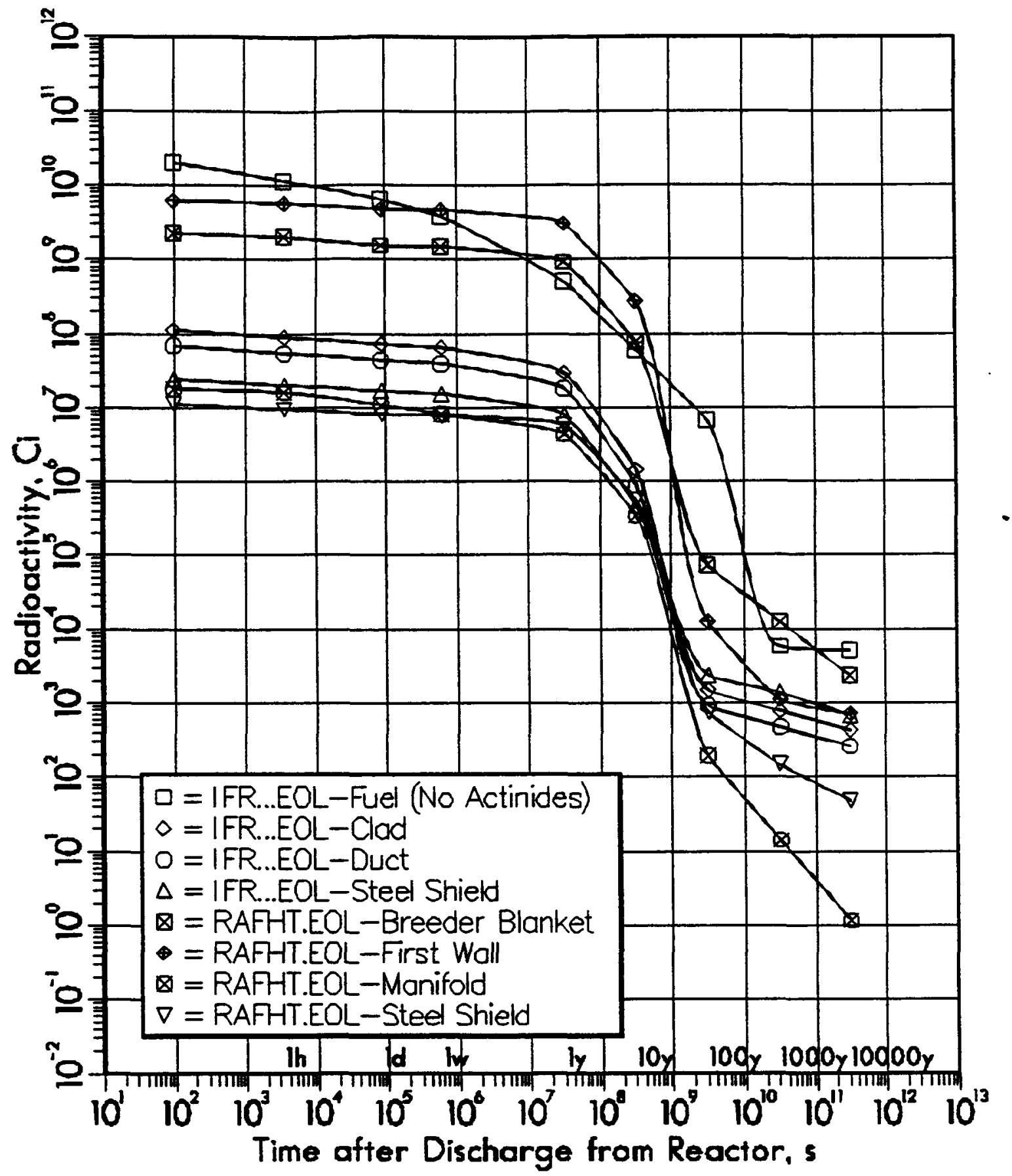

Figure 5.7: Life Time Absolute Radioactivity IFR vs. RAFHT 
clad and duct of EBR-II.

The same reference provides a specific radioactivity of the fuel from a fast fission reactor. This radioactivity shows good agreement with the radioactivity of the fuel from the fast fission reactors as calculated in this study. The latter one is generally within a factor of 2 of the former one. Based on this agreement, it can be assumed that the specific radioactivity of all remaining fast fission reactor components as calculated in this study represents a reasonable estimate.

Reference [11] provides a specific radioactivity of the first wall and the breeder blanket from ITER. This radioactivity shows good agreement with the radioactivity of those components from ITER as calculated in this study. The latter one is generally within a factor of less than 2 of the former one. Based on this agreement, it can be assumed that the specific radioactivity of the RAFHT components as well as the one of all remaining fusion reactor components as calculated in this study represents a reasonable estimate.

All remaining radioactive waste parameters are based on the radioactivity of the components. The different weighting factors of the radionuclides in the components, used to determine those radioactive waste parameters, more or less average themselves out to a constant weighting factor for any component under consideration. The averaging is mostly due to the similar radionuclides in these components.

It is the reason for the fact, that the remaining radioactive waste parameters of the fast fission reactor and fusion reactor components basically show the same temporal behavior as the radioactivity of those components. Furthermore, this means that the order of the components with respect to the magnitude of a radioactive waste parameter does not change significantly with the radioactive waste parameter under consideration. However, radioactive waste parameters of components can be closer together or further apart than the radioactivity, due to slight variations in the avering out.

For these reasons, a description of the temporal behavior shall be forgone 
for the remaining radioactive waste parameters. Rather, particularities shall be noted and the behavior with respect to possible standards for those radioactive waste parameters in order to fulfill certain requirements shall be illustrated.

\subsection{Whole Body $\gamma$-Dose Rate}

The specific whole body $\gamma$-dose rate does not convey much information and therefore its course over time after discharge is not shown here. Much more important is the absolute whole body $\gamma$-dose rate. Its course over time after discharge is shown in Figures 5.8 and 5.9.

The standards of 10CFR20 for restricted areas give a hint to the allowed radiation environment for radiactive waste handling. In general, the radiation environment must not result in a whole body $\gamma$-dose rate greater than $5.7 \cdot 10^{-6} \frac{\mathrm{Sv}}{h}$. Under exceptional circumstances, $1.4 \cdot 10^{-5} \frac{\mathrm{Sv}}{h}$ may be allowed. Only the clad, duct and shield of $E B R-I I$ will reach such a whole body $\gamma$ dose rate, although only within $100 y$ after discharge. All other components do not comply with these standards at any time after discharge.

The same holds true for the $2.5 \cdot 10^{-5} \frac{\mathrm{Sv}}{h}$ to be reached within $100 y$ after discharge as recommended by Reference [39] for long-time hands-on radioactive waste handling. The $2.0 \cdot 10^{-2} \frac{S v}{h}$ to be reached within $50 y$ after discharge as recommended by the same reference for short-time hands-on radioactive waste handling could be fallen short of by all components of $E B R-I I$ within $10 y$ and by all components of $R A F H T$ within several hundred years after discharge. All other components require hands-off, i.e. shielded radioactive waste handling at any time after discharge.

However, self-shielding of the components was not considered and hence the actual whole $\gamma$-dose rate of the components is lower than the idealized one given in the figures. Roughly a self-shielding factor of at least $10^{-4}$ is 
required to bring the whole body $\gamma$-dose rates for all components down to the $10^{-5} \frac{S v}{h}$ range within $10 y$ after discharge.

Whole body $\gamma$-dose weighting factors were available for the radionuclides of activation products and for only a minor part of the radionuclides of fission products. It is estimated that the whole body $\gamma$-dose rate of fuel is by a factor of less than 10 too low due to the lack of whole body $\gamma$-dose weighting factors for a major part of the radionuclides of fission products.

\subsection{Decay Power}

The course of the specific decay power over time after discharge is shown in Figures 5.10 and 5.11. Reference [39] cites a specific decay power of $10 \frac{\mathrm{W}}{\mathrm{m}^{3}}$ to be reached within $50 y$ after discharge in order to allow storage in a repository. With the exception of the fuel and the first wall, all components of $E B R-I I$ and $I T E R$ will fulfill this limitation. Fuel of $E B R-I I$ and first wall of ITER fall short of this limitation at about $100 y$ after discharge almost simultaneously. Also, the specific decay power of $1 \frac{\mathrm{W}}{\mathrm{m}^{3}}$, cited by Reference [39] as virtually negligible, will be reached by almost all components of $E B R$ $I I$ and ITER at several hundred years after discharge. However, this does not hold true for the ITER first wall, which will remain at a specific decay power of greater than $1 \frac{\mathrm{W}}{\mathrm{m}^{3}}$ even at $10,000 y$ after discharge.

The comparison of IFR and RAFHT shows that all components, except for the IFR fuel, will come down to a specific decay power of $10 \frac{\mathrm{W}}{\mathrm{m}^{3}}$ within the above 50y. The fuel needs several hundred years to reach this specific decay power. $1 \frac{\mathrm{W}}{\mathrm{m}^{3}}$ will be fallen short of by all components, except for the fuel, at $100 y$ after discharge and the fuel needs $1,000 y$ to accomplish this.

The course of the absolute radioactivity over time after discharge is shown in Figures 5.12 and 5.13. All components of EBR-II and ITER produce less than $1 M W$ within $1 w$ after discharge. While the fuel of $I F R$ 


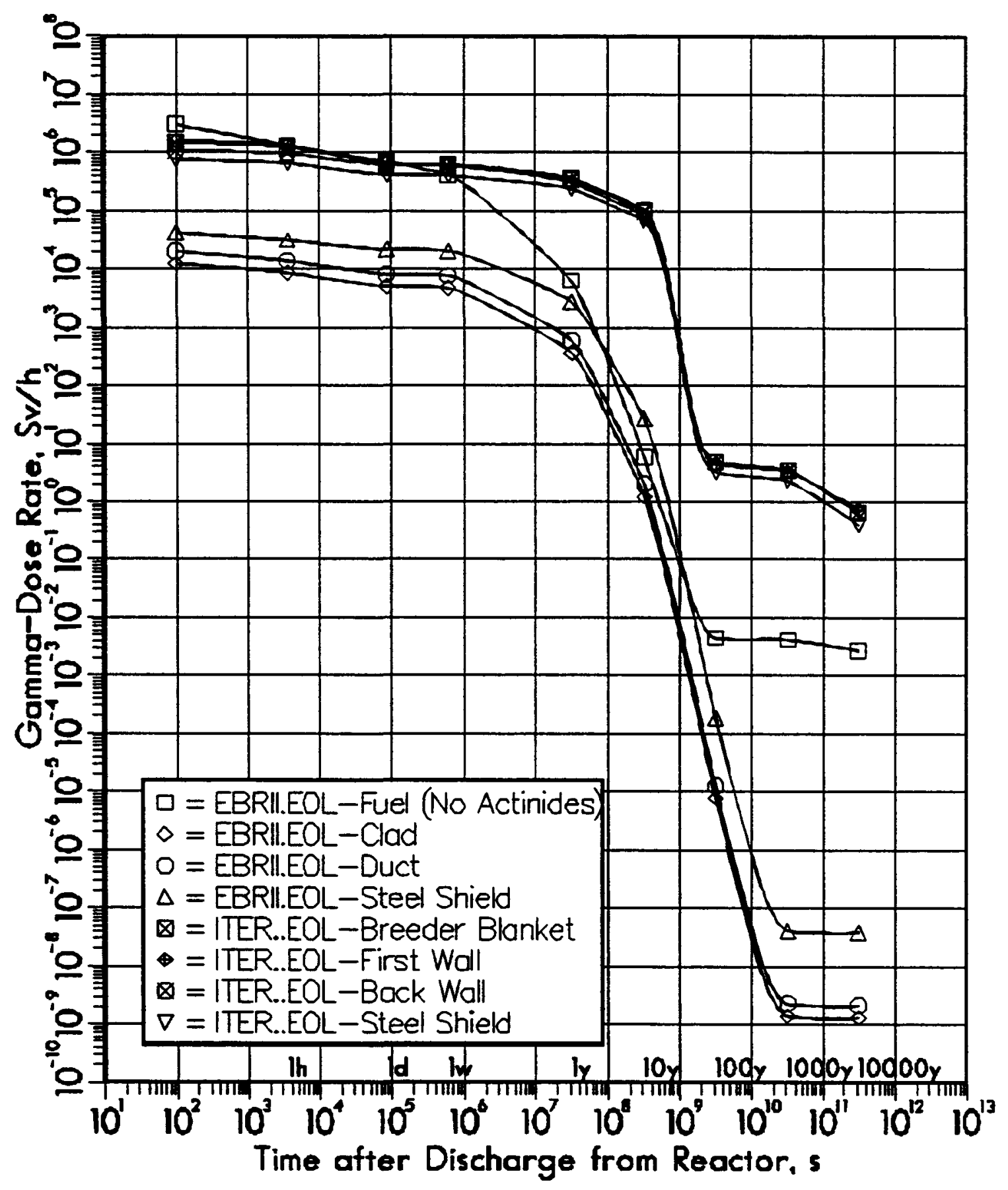

Figure 5.8: Life Time Absolute $\gamma$-Dose Rate EBR-II vs. ITER 


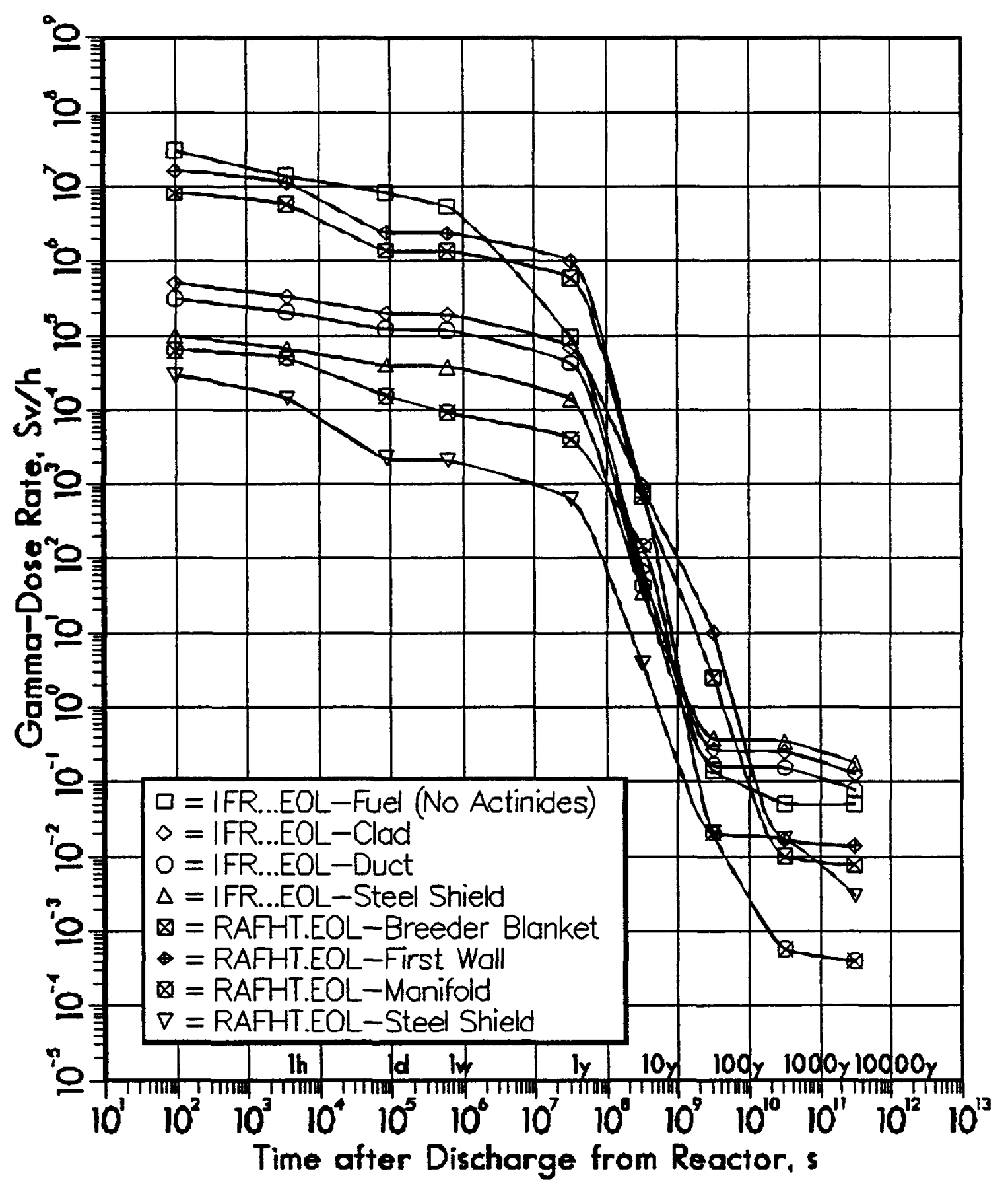

Figure 5.9: Life Time Absolute $\gamma$-Dose Rate IFR vs. RAFHT 


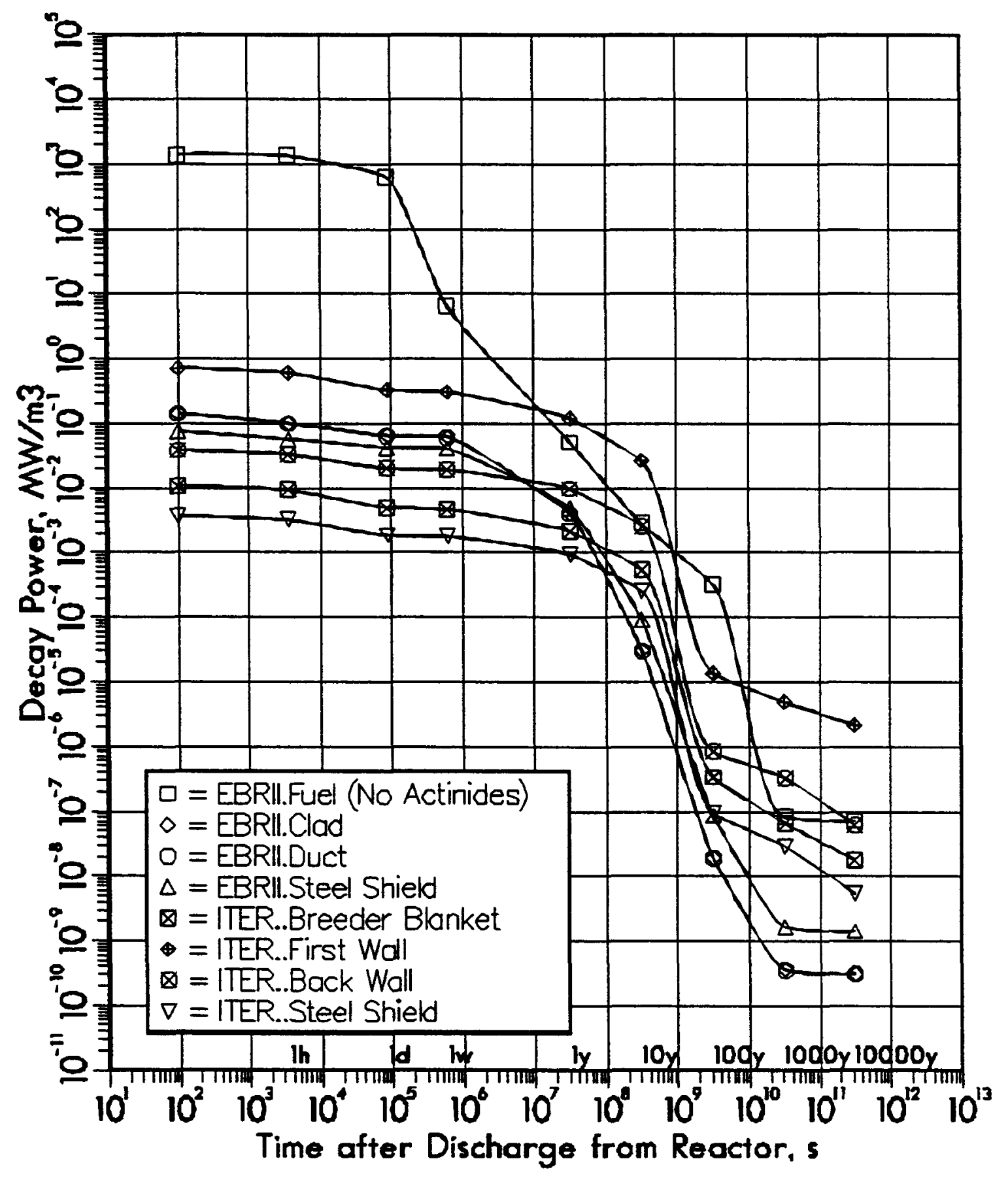

Figure 5.10: Specific Decay Power EBR-II vs. ITER 


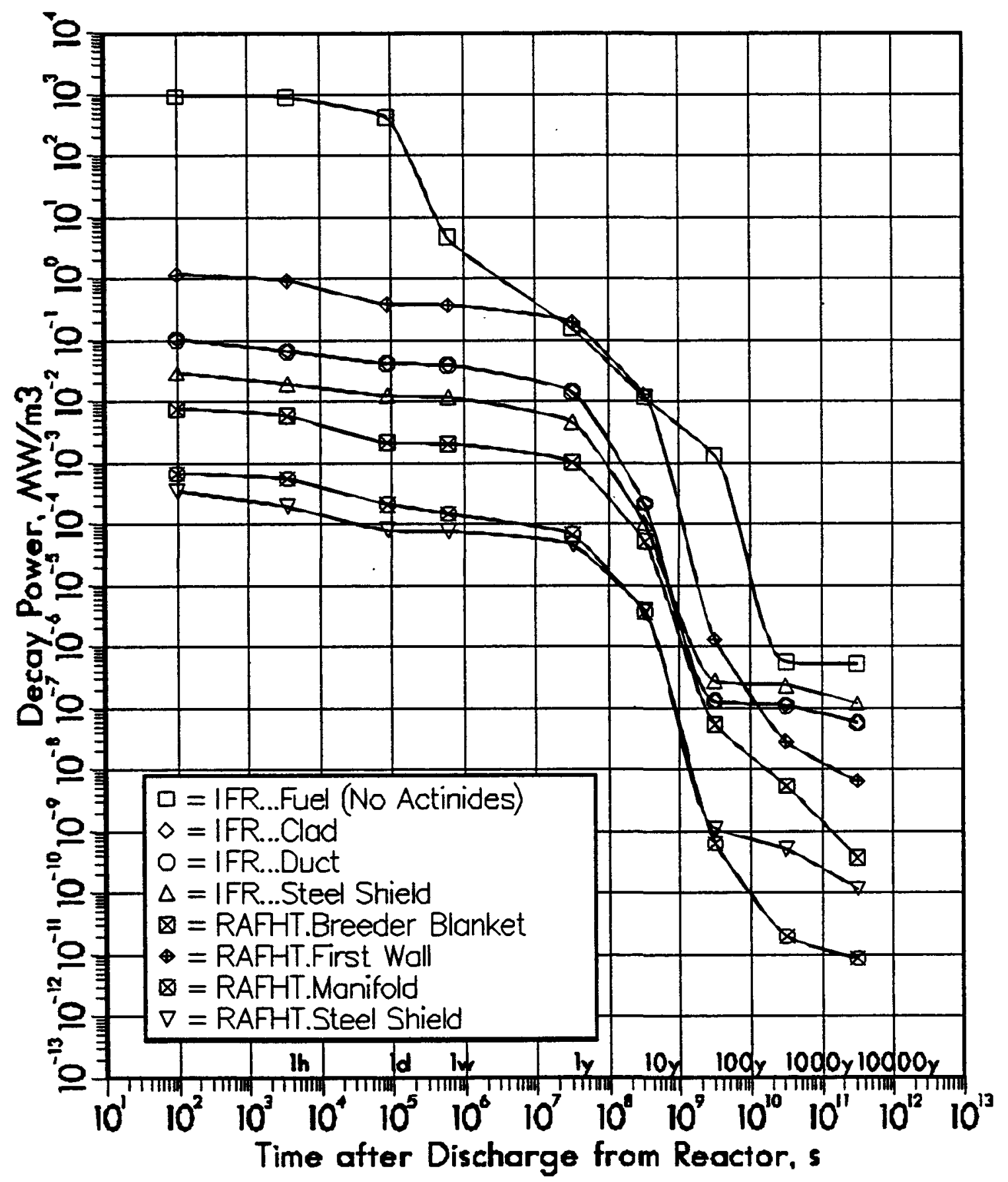

Figure 5.11: Specific Decay Power IFR vs. RAFHT 
and the first wall and breeder blanket of RAFHT need several years to fall short of this absolute decay power, all other components produce less than $1 M W$ right after discharge.

If the absolute decay power of the IFR life time fuel and the RAFHT life time first wall is divided by the number of cycles per life time of the respective reactors, the resulting absolute decay power would still be at $1 M W$ at $1 y$ after discharge. Assuming that, say, 50 reactors of the $I F R$ and $R A F H T$ type each are existing at a future point in time, the combined absolute decay power of the cycle fuel and the cycle first wall would make up for more than $100 M W_{t h}$, i.e. the size of a small conventional power station, for $1 y$. However, the efficiency of conversion from thermal to electrical power output is relatively low. Also, using the first wall as an energy source would interfere with the intention of reprocessing it, while the fuel is already reprocessed and hence suitable as a heat source.

Even more so than for the absolute radioactivity it is striking how concentrated the absolute decay power for the ITER components appears in the graph.

While radionuclides of activation products are only a few neutrons away from their equilibrium condition in terms of the number of neutrons, radionuclides of fission products are sometimes more than 10 neutrons away from their equilibrium condition. This results in a much higher heat production for the decay of a radionuclide of fission products than for the one of a radionuclide of activation products.

Consequently a much higher specific and absolute decay power results for fuel than for the remainder of the components. That also means that fuel is especially "hot" for the first couple of days after discharge. However, both specific and absolute decay power come down to the decay power of the first wall at less than $1 y$ after discharge. The graph for fuel starts to fall off at $1 d$ after discharge already, displaying the fact that the short-lived radionuclides decay soon. 


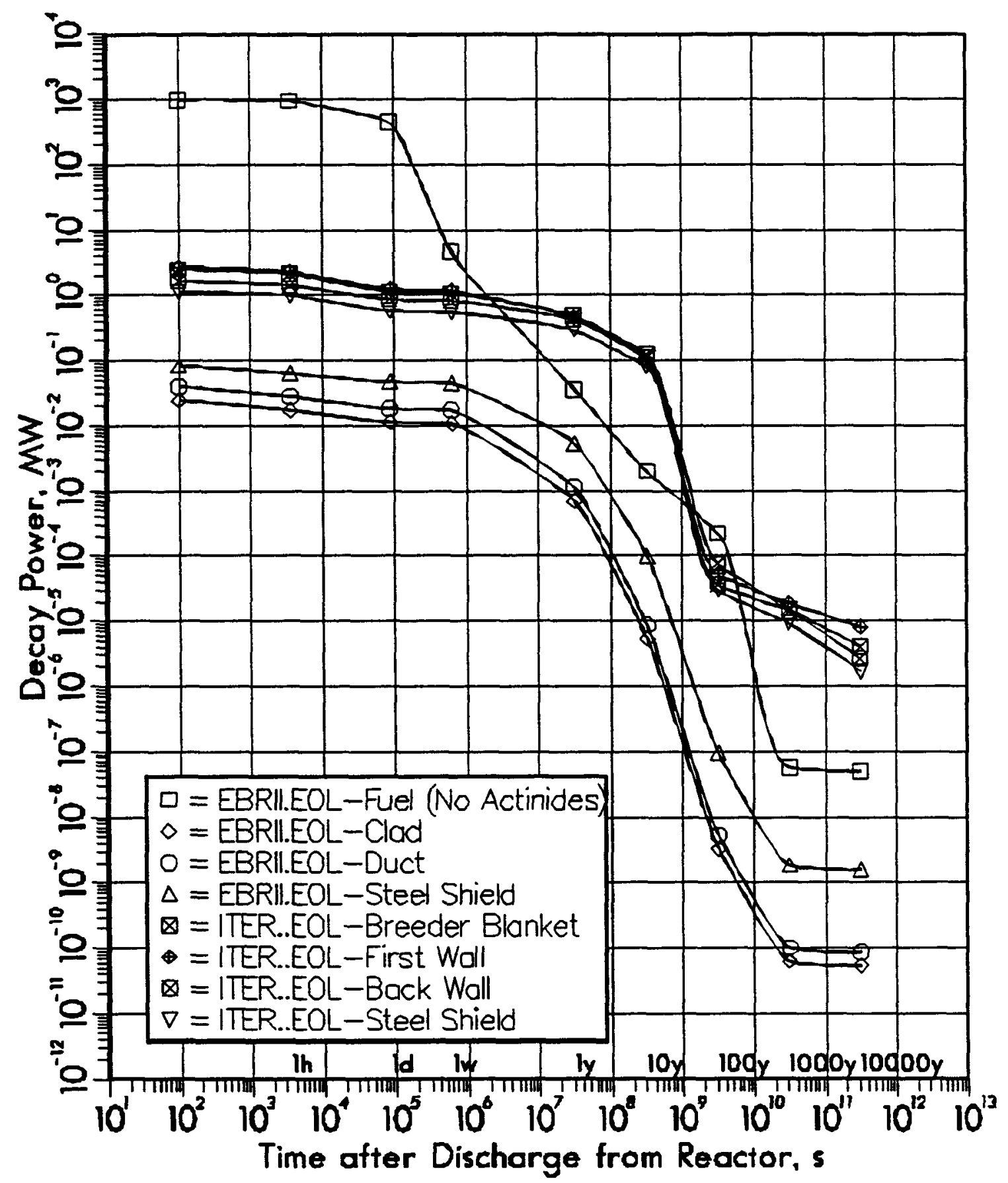

Figure 5.12: Life Time Absolute Decay Power EBR-II vs. ITER 


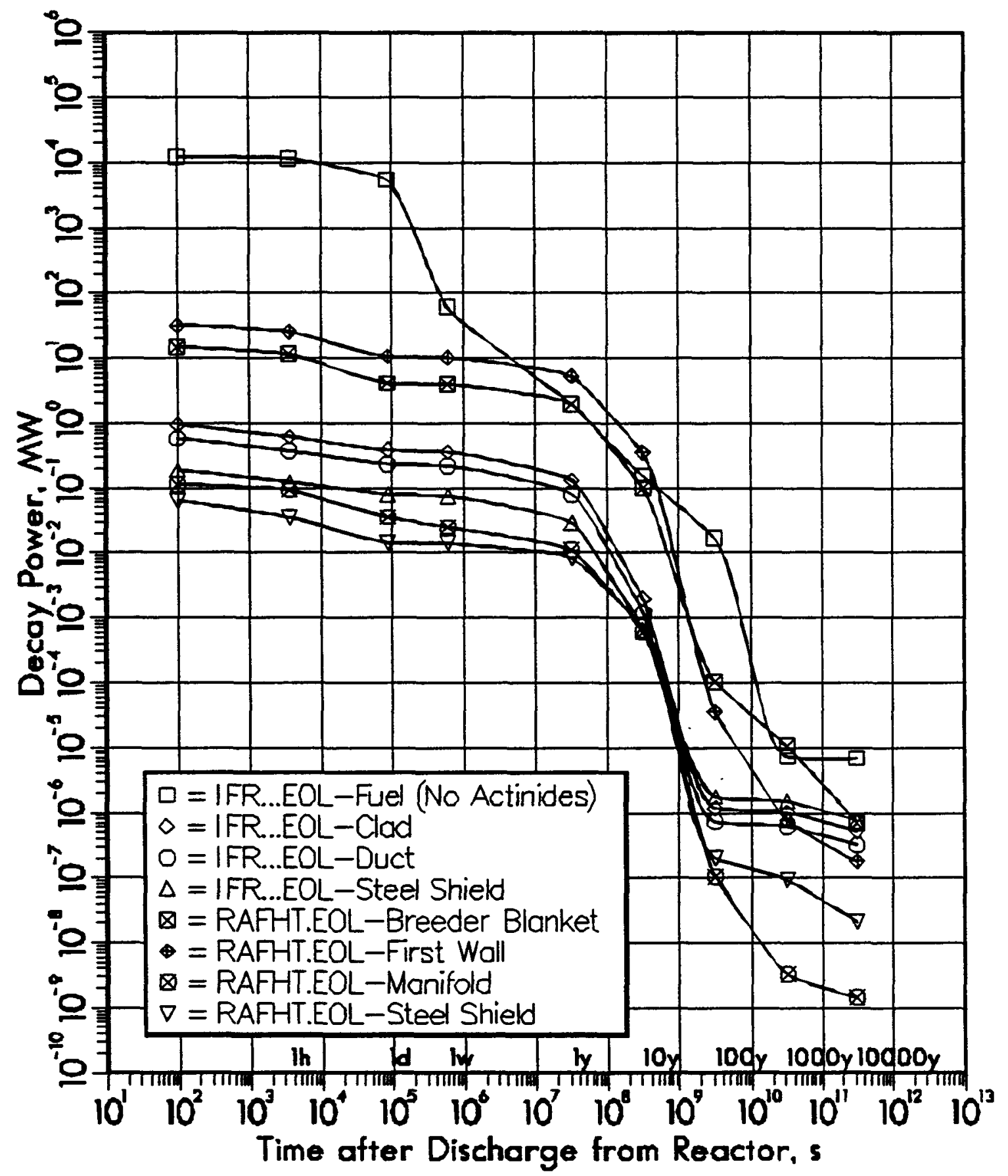

Figure 5.13: Life Time Absolute Decay Power IFR vs. RAFHT 


\subsection{Biological Hazard Potential}

The course of the specific Biological Hazard Potential over time after discharge is shown in Figures 5.14 and 5.15. It can be seen that no component of any reactor reaches a specific $B H P$ equal to unity within 10,000y. All components therefore represent a serious hazard to the biosphere for a very long time.

The course of the absolute Biological Hazard Potential over time after discharge is shown in Figures 5.16 and 5.17. A supertanker fleet of about 4,000 ships represents the immense volume of $1 \mathrm{~km}^{3}$. It takes the absolute $B H P$ of the $E B R-I I$ fuel several hundred years and each one of the remainder of the $E B R-I I$ components several years to fall short of this volume. The absolute $B H P$ of all ITER components reaches this volume only at several thousand years after discharge.

Except for the IFR fuel and the RAFHT first wall, all components of IFR and $R A F H T$ drop below the $1 \mathrm{~km}^{3}$ volume at $10 y$ after discharge. While $I F R$ fuel and $R A F H T$ first wall reach this volume after several hundred years after discharge, it takes the RAFHT breeder blanket several thousand years to accomplish this; it is mostly caused by the low $M P C$ of the radionuclides ${ }^{14} \mathrm{C}$ and ${ }^{39} \mathrm{Ar}$ produced in the breeder material.

\subsection{Radioactive Waste Classification}

Note that the classification presented here is pertinent to US regulatory standards. Countries other than the US might have different standards, which might lead to a different classification. Also note that according to $10 C F R 61$, radioactive waste that does not fall into class $A, B$ or $C$ is not necessarily high level radioactive waste. However, for the purpose of the study, this waste is assumed to be high level radioactive waste, because it exceeds the limits for class $A, B$ or $C$ waste by a large factor in almost all 


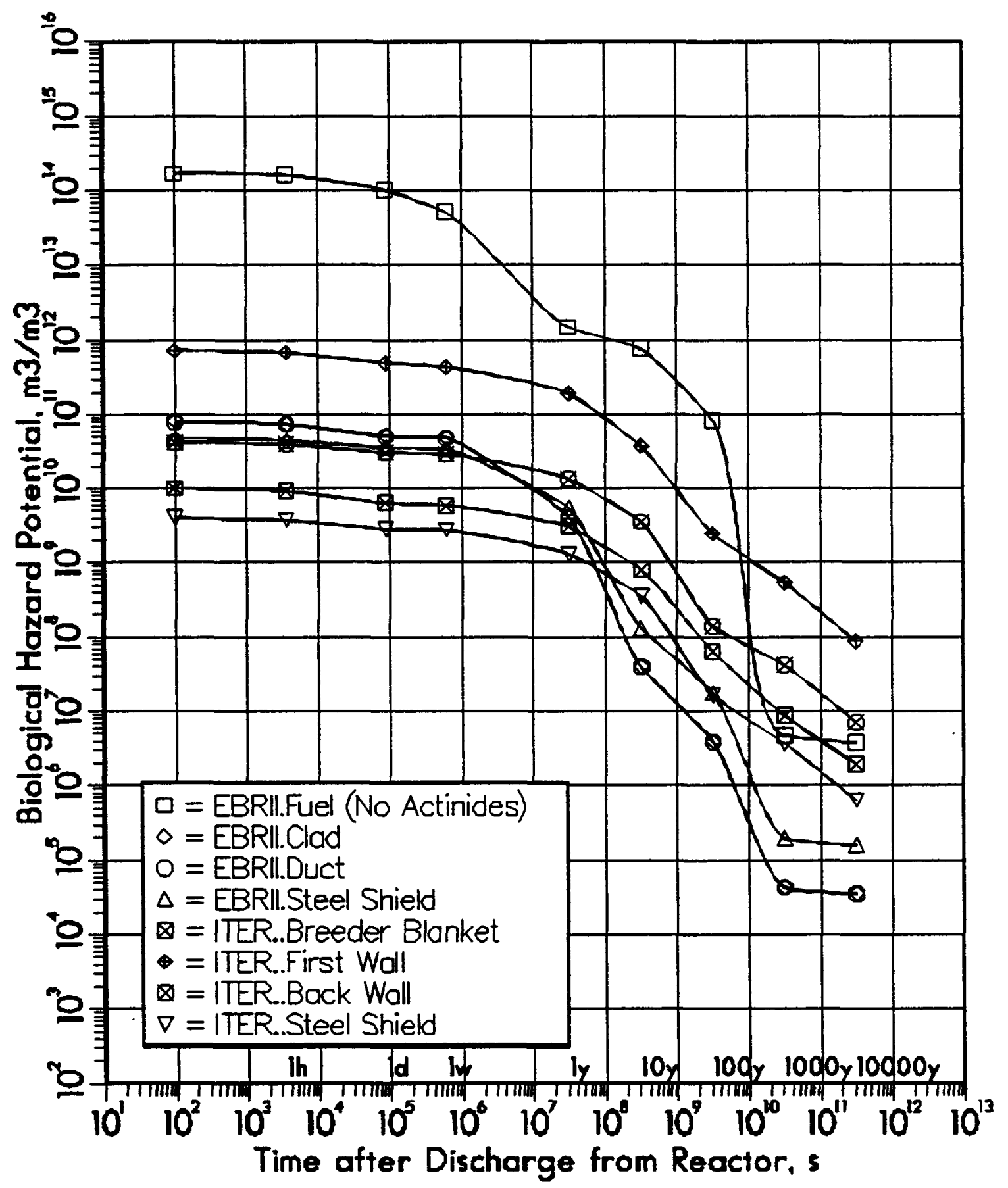

Figure 5.14: Specific Biological Hazard Potential EBR-II vs. ITER 


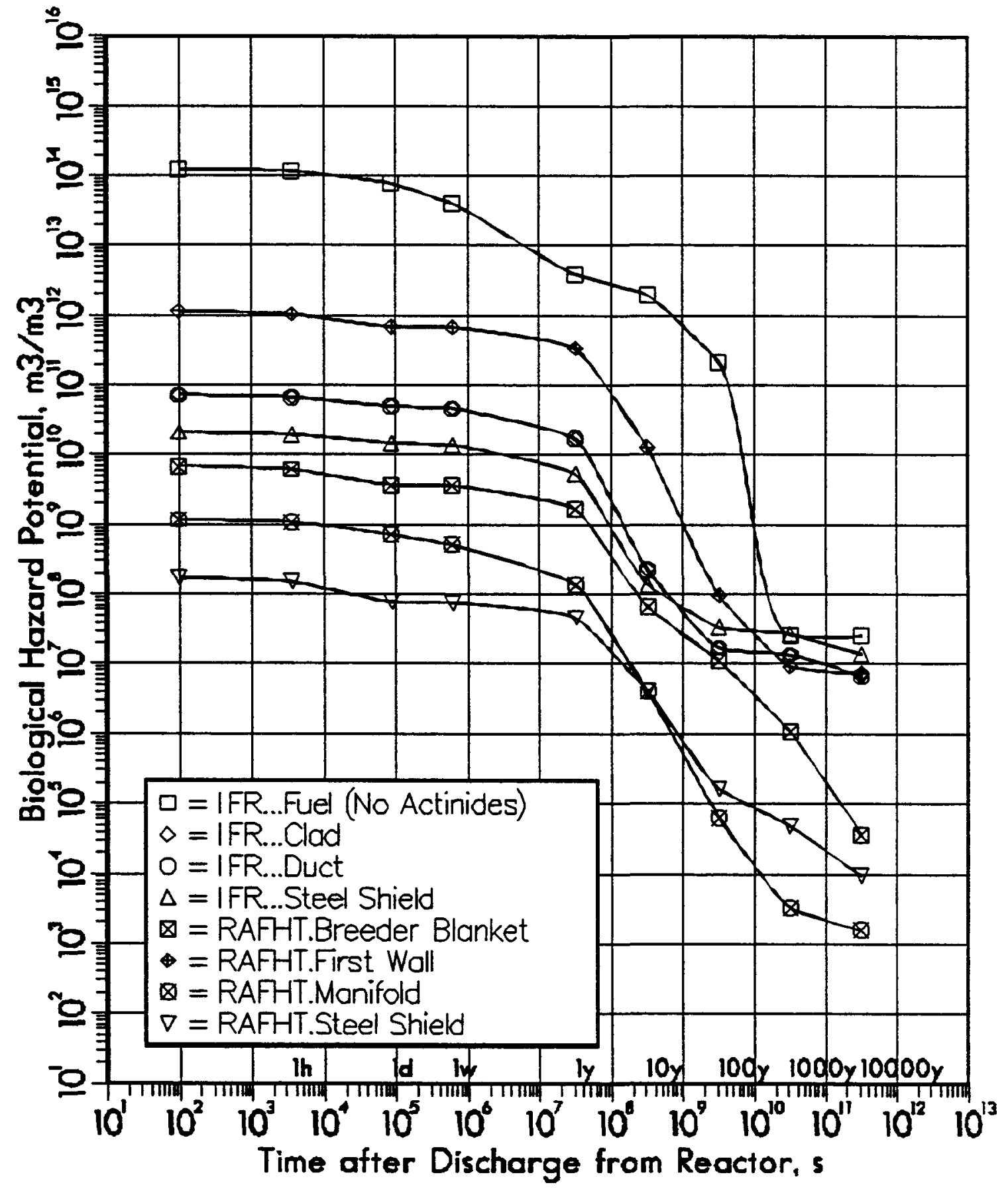

Figure 5.15: Specific Biological Hazard Potential IFR vs. RAFHT 


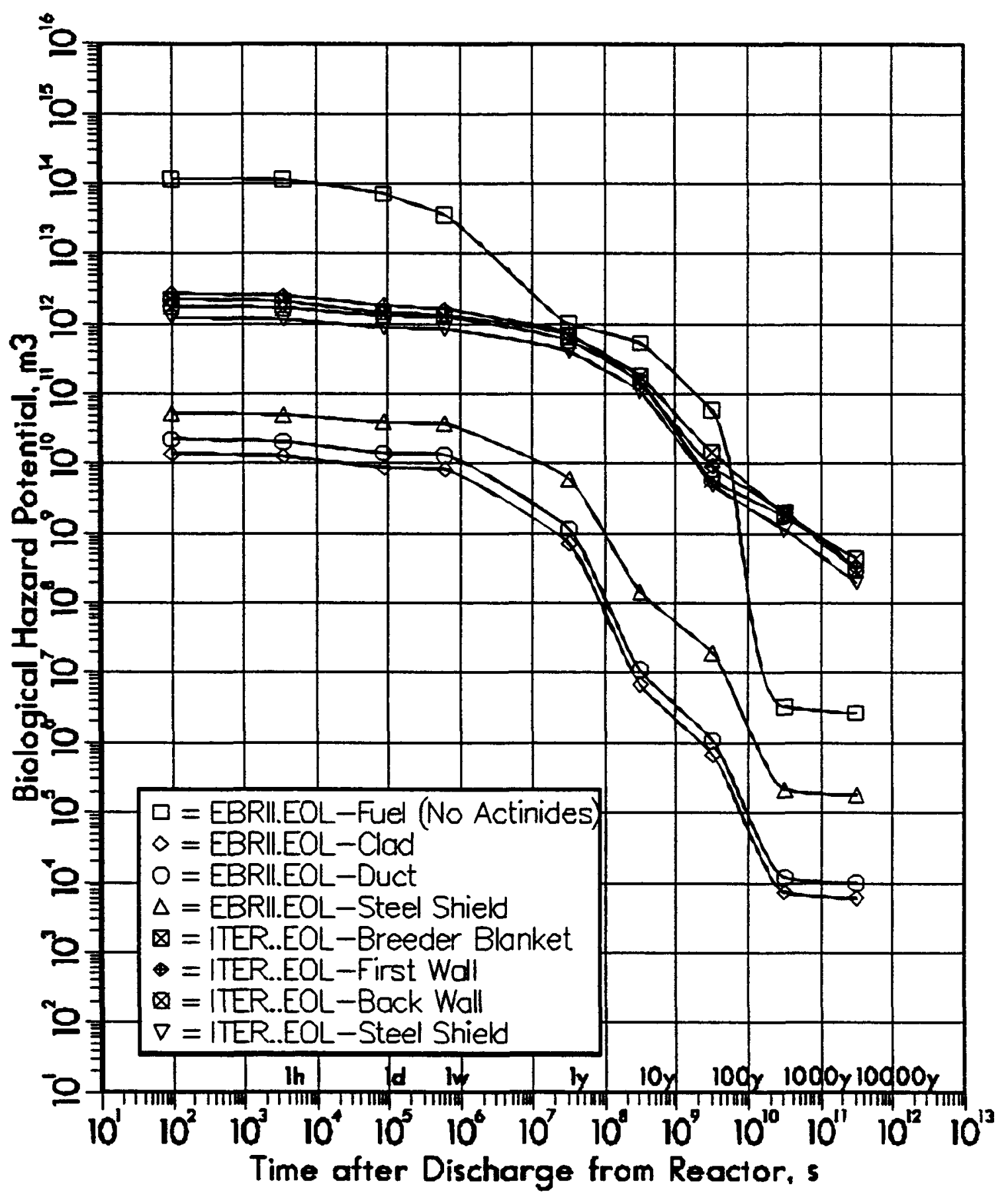

Figure 5.16: Life Time Absolute Biological Hazard Potential EBR-II vs. ITER 


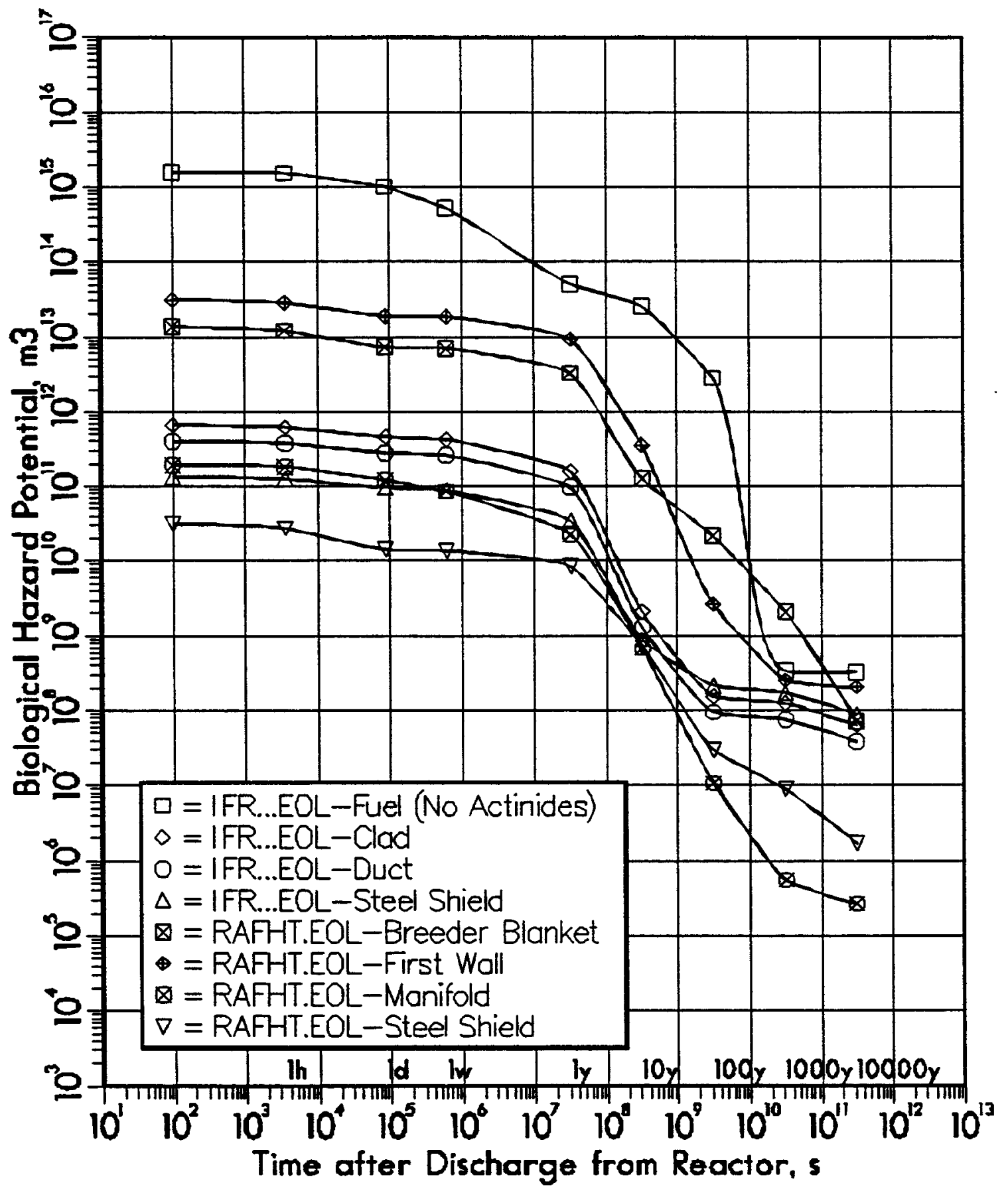

Figure 5.17: Life Time Absolute Biological Hazard Potential IFR vs. $R A F H T$ 
cases. The association of high level radioactive waste with deep repositories or low and intermediate level radioactive waste with shallow repositories is based on typical considerations in radioactive waste management but is by no means compelling.

The course of the classification index $R W C_{C}$ for radioactive waste class $C$ over time after discharge is shown in Figures 5.18 and 5.19. Any component with $R W C_{C}$ greater than 1 has to be classified as high level radioactive waste, while $R W C_{C}$ less than 1 qualifies the component for class $C$ intermediate level radioactive waste or even lower classes $B$ and $A$ radioactive waste.

Clad, duct and shield of $E B R-I I$ clearly qualify for class $C$ radioactive waste right after discharge and only the fuel has to be classified as high level radioactive waste. In contrast, first wall, breeder blanket and back wall of ITER constitute high level radioactive waste for 10,000years after discharge and only the shield qualifies for class $C$ intermediate level radioactive waste right after discharge. However, $R W C_{C}$ s of back wall and breeder blanket are greater than 1 by less than a factor of two, which in view of possible uncertainties in the calculation means that both components could also qualify for class $C$ intermediate level radioactive waste, implying that only the fuel of $E B R-I I$ and the first wall of ITER must be stored in deep repositories.

All components of IFR have to be classified as high level radioactive waste right after discharge, but clad and duct fall below an $R W C_{C}$ classification index of 1 at $1 y$ after discharge. The $R W C_{C}$ classification index of the steel shield almost reaches unity at $1 y$ after discharge, with the same caveat as for the ITER back wall and breeder blanket. The first wall is the only component of $R A F H T$ that possibly could be high level radioactive waste for more than 10,000y after discharge; but again its $R W C_{C}$ classification index for class $C$ radioactive waste is only slightly greater than one. All other components of $R A F H T$ qualify for class $C$ intermediate level radioactive waste. 


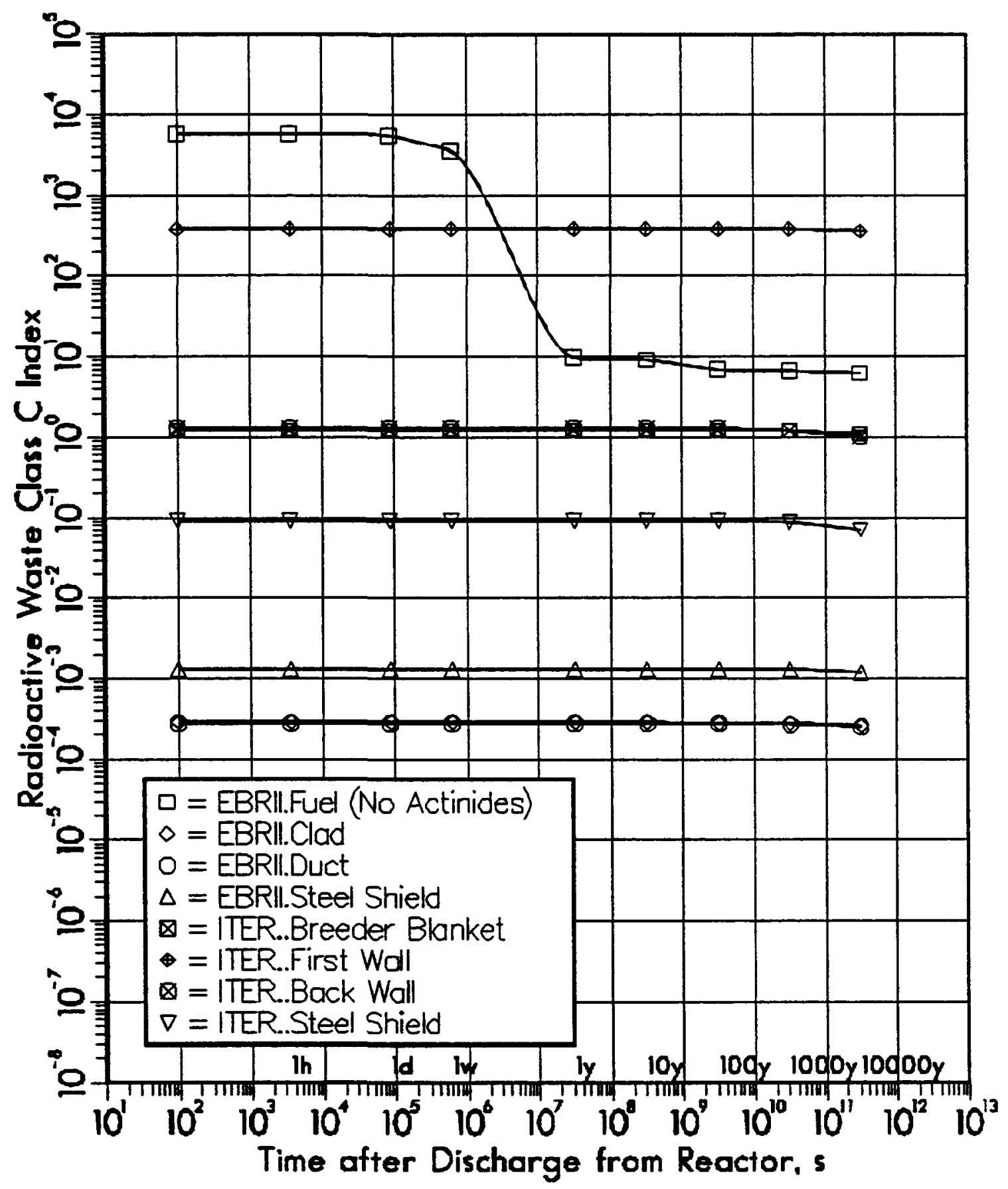

Figure 5.18: Radioactive Waste Class $C$ Index, EBR-II vs. ITER 


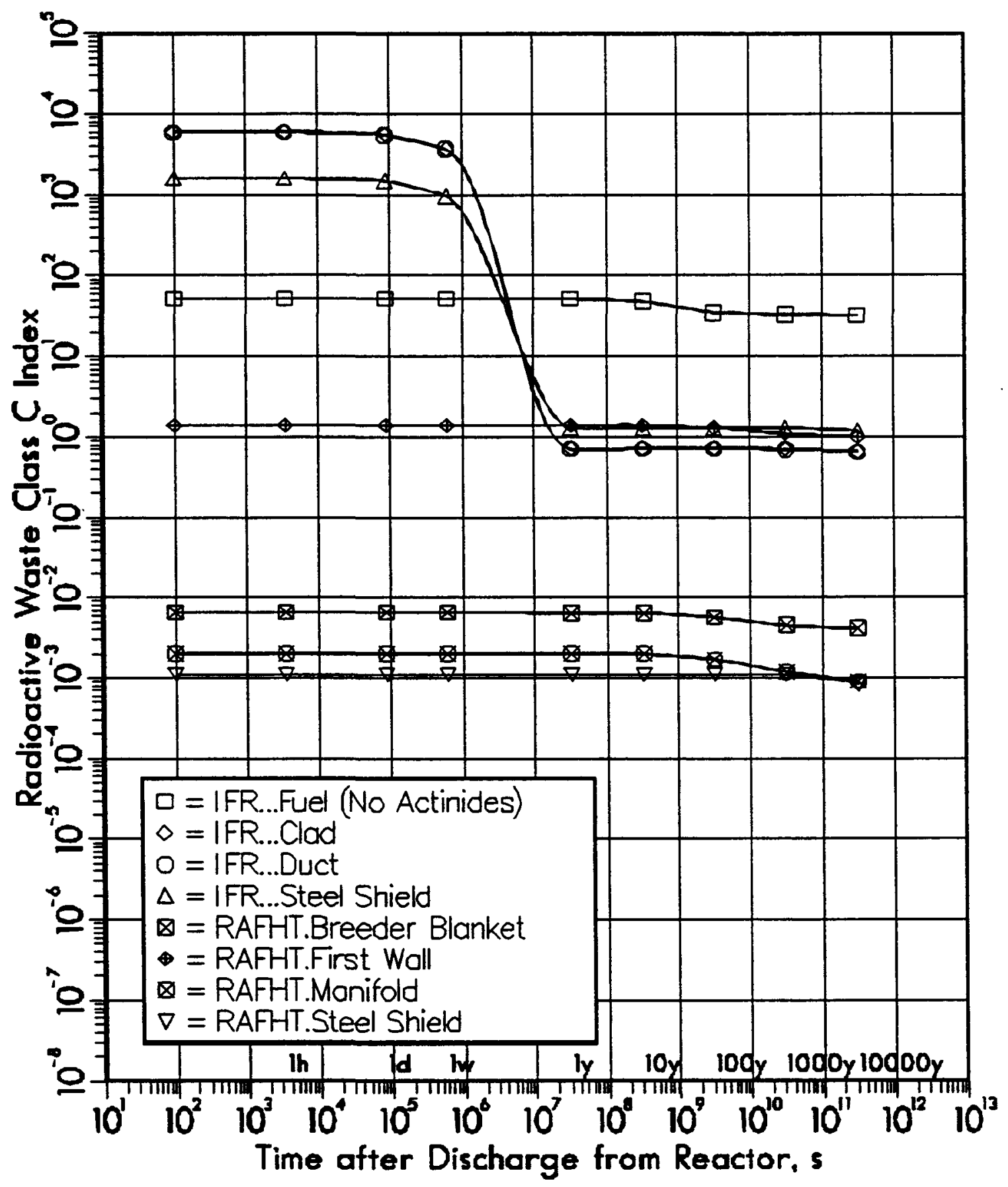

Figure 5.19: Radioactive Waste Class $C$ Index, IFR vs. RAFHT 
The strange behavior of the $E B R-I I$ fuel as compared to $I F R$ fuel is most likely due to the different specific radioactivity of the short-lived radionuclide ${ }^{92} \mathrm{Nb}$, which is partly produced by $\mathrm{Nb}$ initially present in $E B R-I I$ fuel, while there is no initial $N b$ present in $I F R$ fuel. The same $N b$ isotope is probably responsible for the likewise strange behavior of clad, duct and steel shield of $I F R$ as compared to $E B R$-II, i.e. there is ${ }^{92} \mathrm{Nb}$ present in those components of $I F R$ but not of $E B R-I I .{ }^{92} \mathrm{Nb}$ is partly produced by radioactive decay of activated $M o$; IFR clad, duct and shield being made of $H T-9$ do have some $M o$, while the clad duct and shield material $S S 304 L$ of $E B R-I I$ does not contain $M o$. Due to the particular low WDR of this $N b$ isotope and the generally low number of relevant radionuclides considered for radioactive waste classification purposes, ${ }^{92} \mathrm{Nb}$ can be the determining radionuclide with respect to radioactive waste classification.

There is, however, a possibility that the radionuclide specified as ${ }^{92} \mathrm{Nb}$ in the ORIGEN-II decay data library, which was obtained from Argonne National Laboratory [14], is actually supposed to be the $N b$ isotope ${ }^{82 m} \mathrm{Nb}$. In that case it would not matter for classification purposes and the graphs for the $R W C_{C}$ classification index would run as straight lines, classifying clad and duct of $I F R$ as class $C$ intermediate level radioactive waste right after discharge and leaving only the $I F R$ fuel and steel shield as high level radioactive waste. Also, the $E B R$-II fuel would remain high level radioactive waste under this assumption with the corresponding graph running as a straight line.

Should this explanation hold true, and this is most likely the case, as a comparison of half life and radionuclide between the ORIGEN-II decay data library and the Table of Isotopes suggests, then it demonstrates the sensitivity of the radioactive waste classification method.

The above uncertainty about the $N b$ isotopes ${ }^{92} \mathrm{Nb}$ and ${ }^{92 m} \mathrm{Nb}$, respectively, was reported to Argonne National Laboratory, but no final clarifica- 
tion of this uncertainty was obtained [14].

\subsection{Intruder Dose Rate}

\subsubsection{Construction Scenario}

For the purpose of this study, a layered waste repository design was chosen. This results in additional shielding equivalent to a certain thickness of soil between the radioactive waste and the intruder. The shielding effect depends on the radionuclide and is assumed to be effective for the time the layered waste repository design allows stable waste packages to exist - here $500 y$ after beginning of storage. After this period, the shielding is stated to be not effective anymore.

The shielding explains the typical shape of the graph of the intruder dose rate versus time after beginning of storage. The decrease due to natural decay of radionuclides is enhanced by the shielding effect until $500 y$ after beginning of storage, leading the graph into a "trough". Then the shielding effect vanishes and the graph increases almost instantaneously to a "peak" before starting to fall off again, this time much slower than previously with shielding. The graph distorts reality a little bit, because radioactive waste parameters for $500 y$ were not determined and thus the increase seemingly already starts at $100 y$ after beginning of storage,i.e. the time at which institutional control is assumed to cease to exist. Radioactive waste with radionuclides whose radiation does not or only weakly experience a significant shielding does not or only much weaker exhibit the "peak" and "trough" behavior.

The course of the intruder dose rate under the construction scenario over time after discharge is shown in Figures 5.20 and 5.21.

The graphs displayed in above figures follow precisely this description, and the graphs for fuel do not go through a significant "trough", because its intruder dose rate is determined by ${ }^{90} \mathrm{Sr},{ }^{90} \mathrm{Y},{ }^{93} \mathrm{Zr},{ }^{99} \mathrm{Tc},{ }^{121 \mathrm{~m}} \mathrm{Sn},{ }^{129} \mathrm{I}$, 


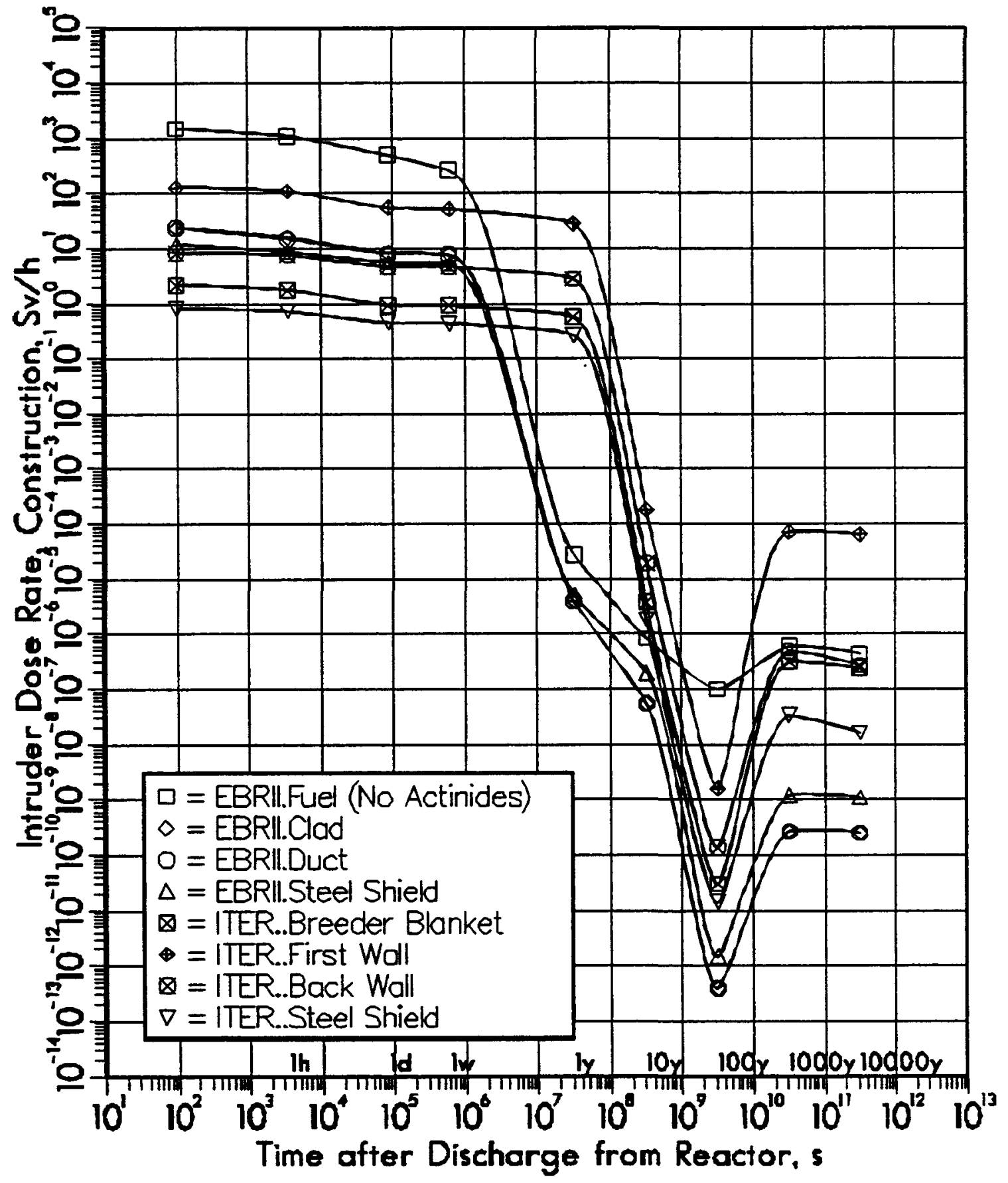

Figure 5.20: Intruder Dose Rate, Construction Scenario, EBR-II vs. ITER 


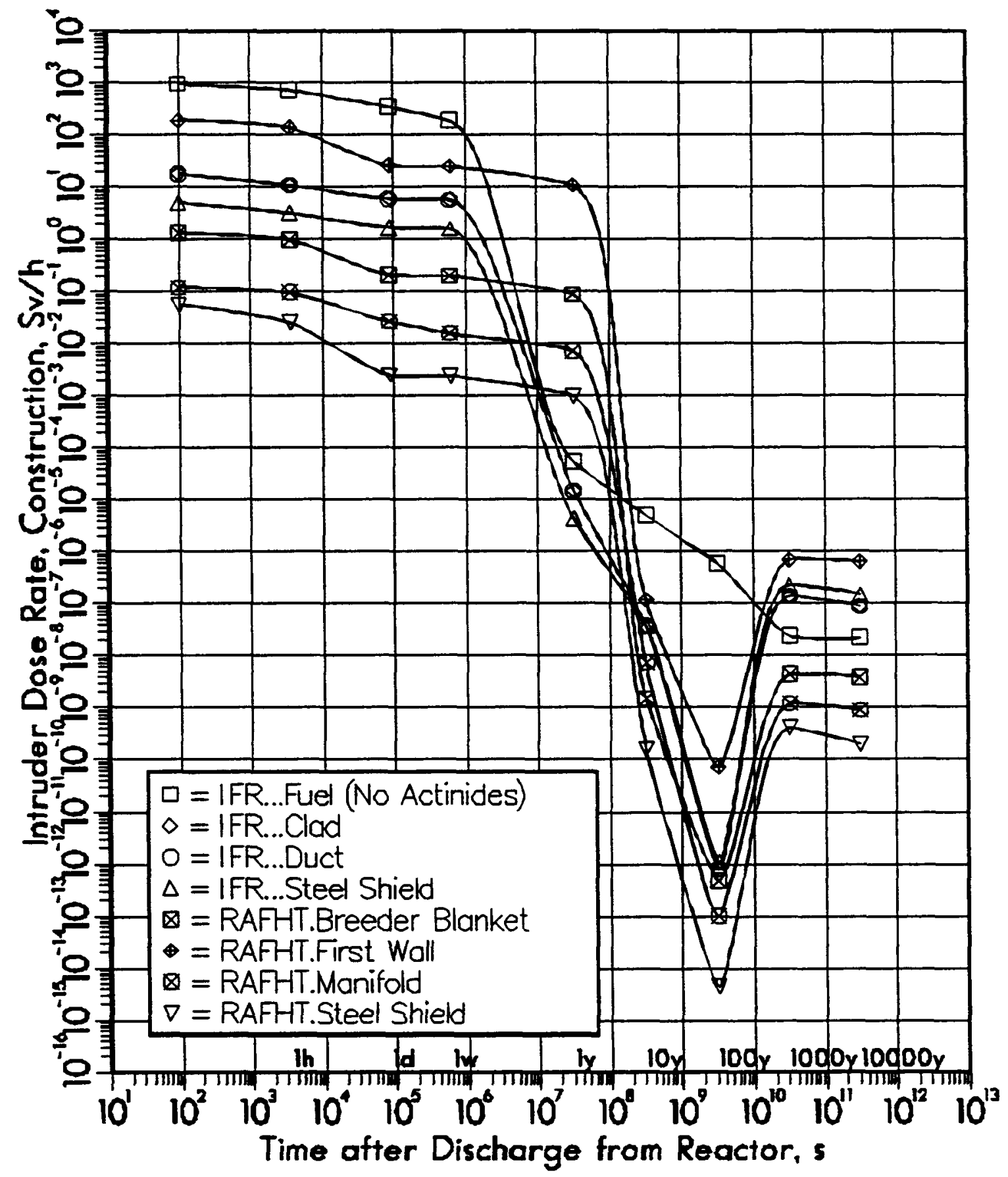

Figure 5.21: Intruder Dose Rate, Construction Scenario, IFR vs. RAFHT 
${ }^{93 m} \mathrm{Nb}$ and ${ }^{137} \mathrm{Cs}$, which, with the exception of ${ }^{137} \mathrm{Cs}$, experience no shielding [8].

Of all high level radioactive waste components only the first wall of ITER significantly exceeds the standard of $5.7 \cdot 10^{-7} \frac{\mathrm{Sv}}{\mathrm{h}}$ for unrestricted areas set by 10CFR20. Back wall and breeder blanket of ITER and first wall of $R A F H T$ as well as fuel of $E B R-I I$ and $I F R$ approach this standard from the lower side or just touch it. In particular it should be noted that, except for the first wall of ITER, no component at any time after institutional control ceases to exist will lead to an intruder dose rate greater than the 10CFR20 standard. Thus it should be possible to store this high level radioactive waste in a shallow repository.

\subsubsection{Agriculture Scenario}

The course of the intruder dose rate under the agriculture scenario over time after discharge is shown in Figures 5.22 and 5.23. Waste packages are assumed to be unstable under this scenario right after institutional control ceases to exist, and hence there is no shielding effect due to a particular repository design. This, in combination with the food pathway in addition to the air and soil pathways of the construction scenario, leads to a higher intruder dose rate than under the construction scenario.

All components classified as high level radioactive waste exhibit an intruder dose rate greater than the 10CFR20 standard. As a discrepancy to their classification, also clad and duct of IFR and steel shield of ITER exceed this standard. This is acceptable in the case of clad and duct, since they classify for class $C$ radioactive waste with a $R W C_{C}$ classification index of only slightly less than 1 and hence exist in the "grey zone". The steel shield however falls short of $R W C_{C}=1$ by almost an order of magnitude. ${ }^{92} \mathrm{Nb}$ or ${ }^{92 \mathrm{~m}} \mathrm{Nb}$ can not be made responsible for this discrepancy, because both $N b$ isotopes have approximately the same $P D C$ and shielding factors.

A remark may be made on the relation of radioactive waste classification 


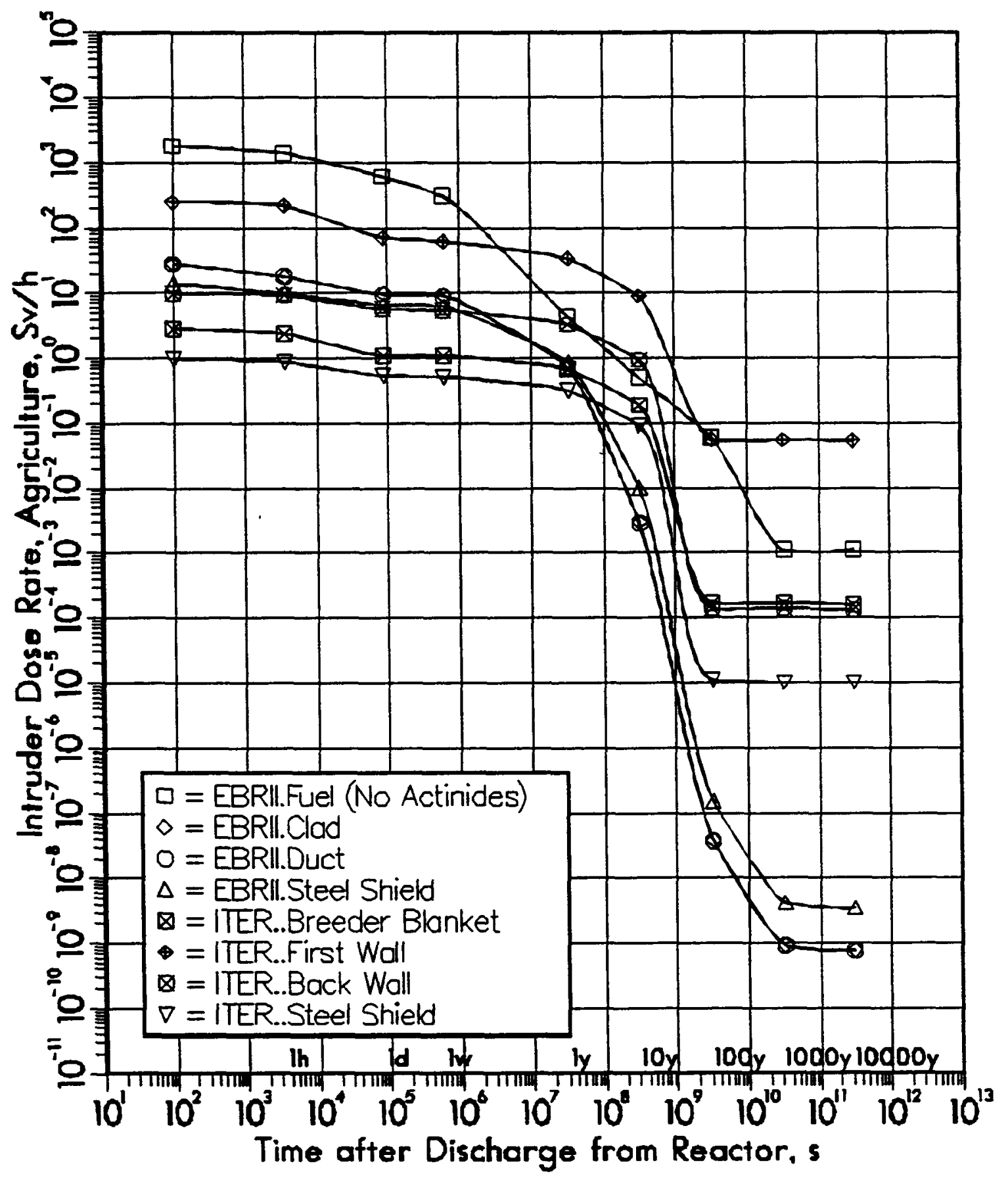

Figure 5.22: Intruder Dose Rate, Agriculture Scenario, EBR-II vs. ITER 


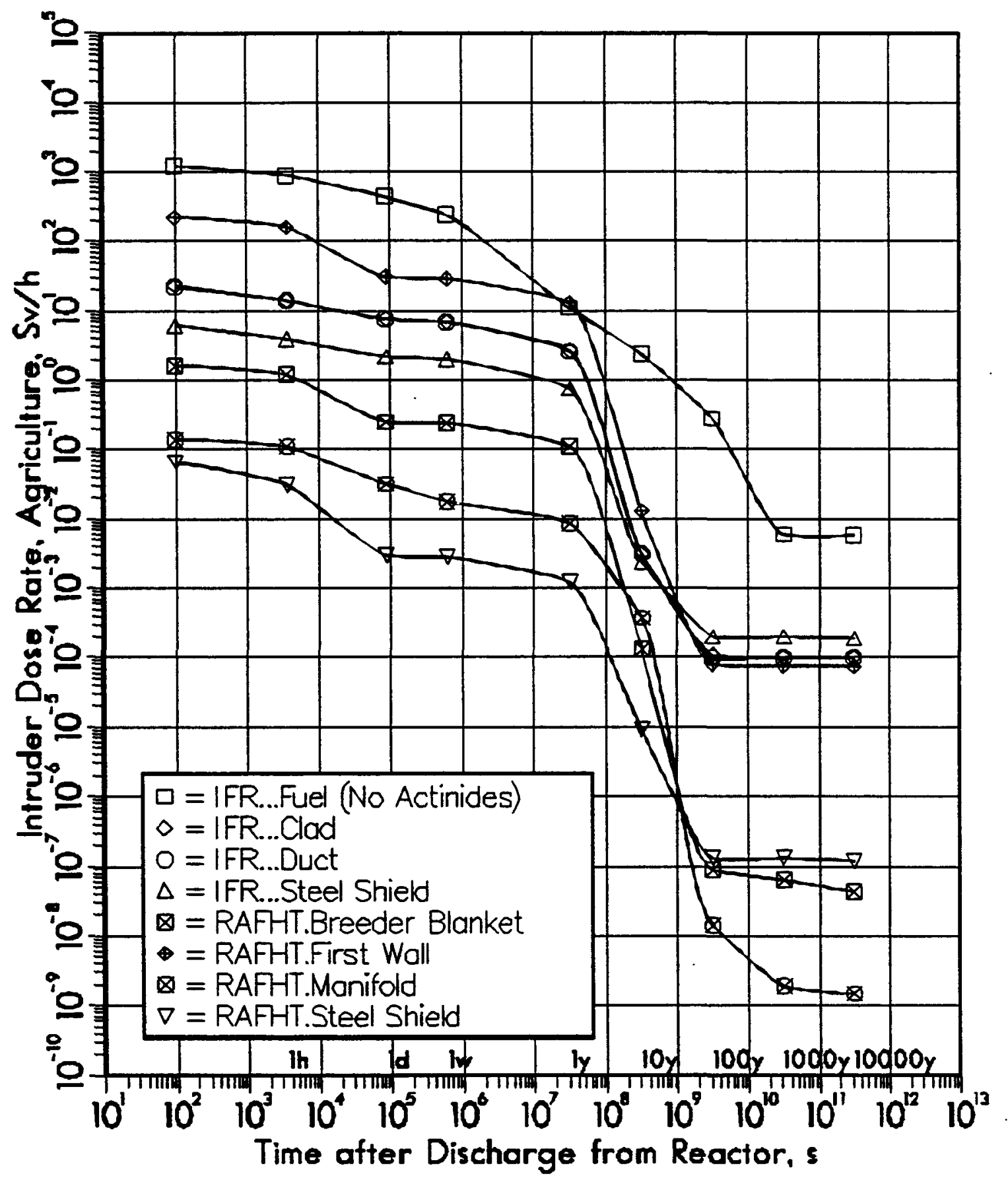

Figure 5.23: Intruder Dose Rate, Agriculture Scenario, IFR vs. RAFHT 
and intruder dose rate. It appears that a safe radioactive waste classification into high level radioactive waste and intermediate or low level radioactive waste that correctly corresponds to the intruder dose rate can be made only, if unity for the $R W C_{C}$ classification index is fallen short of by at least an order of magnitude.

\subsection{Radionuclides in Radioactive Waste}

The components in comparison contain different materials, have different geometries and are irradiated by a different neutron flux. However, the strongest influence on activation is exercised by the materials. To help associate particular high or low radioactive waste parameters with particular radionuclides in those materials, this section lists the most important radionuclides for each component. The importance of the single radionuclides changes over time and the following list is representative for $10 y, 100 y$ and $1,000 y$ after discharge only. It turns out that all radioactive waste parameters for a component are ruled by basically the same radionuclides.

Clad, duct and shield in EBR-II are made of SS304L stainless steel with a particular high $\mathrm{Ni}$ content. Activation of these components is therefore mostly due to ${ }^{59} \mathrm{Ni}$ and ${ }^{63} \mathrm{Ni}$. Other important activation products in $S S 304 \mathrm{~L}$ are ${ }^{54} \mathrm{Mn},{ }^{55} \mathrm{Fe}$ and ${ }^{60} \mathrm{Co}$.

Fuel in $E B R-I I$ is a compound of uranium, plutonium and fissium. The fission products from fission of uranium and plutonium comprise ${ }^{79} \mathrm{Se},{ }^{90} \mathrm{Sr}$, ${ }^{90} \mathrm{Y},{ }^{93} \mathrm{Zr},{ }^{93 m} \mathrm{Nb},{ }^{99} \mathrm{Tc},{ }^{108 m} \mathrm{Ag},{ }^{121 m} \mathrm{Sn},{ }^{125} \mathrm{Sb},{ }^{126 m} \mathrm{Sb},{ }^{125 m} \mathrm{Te},{ }^{129} \mathrm{I},{ }^{137} \mathrm{Cs}$, ${ }^{137 m} \mathrm{Ba},{ }^{93 m} \mathrm{Nb},{ }^{94} \mathrm{Nb},{ }^{93} \mathrm{Mo}$ and ${ }^{99} \mathrm{Tc}$ and the most important activation products due to fissium are ${ }^{93 m} \mathrm{Nb},{ }^{94} \mathrm{Nb},{ }^{93} \mathrm{Mo}$ and ${ }^{99} \mathrm{Tc}$.

Clad, duct and shield in IFR are made of $H T-9$ stainless steel which has only few $\mathrm{Ni}$ but therefore some $\mathrm{Mo}$. Consequently, ${ }^{93 m} \mathrm{Nb},{ }^{94} \mathrm{Nb},{ }^{93} \mathrm{Mo}$ are most important, then follow ${ }^{54} \mathrm{Mn},{ }^{55} \mathrm{Fe},{ }^{60} \mathrm{Co},{ }^{59} \mathrm{Ni},{ }^{63} \mathrm{Ni}$ and ${ }^{99} \mathrm{Tc}$. The $B_{4} C$ part of the shield generates some ${ }^{14} C$. 
The fission products of $I F R$ fuel are not significantly different to $E B R$ $I I$ fuel. But IFR fuel is a compound of uranium, plutonium, actinides and zirconium and the $\mathrm{Zr}$ leads to ${ }^{90} \mathrm{Sr},{ }^{90} \mathrm{Y},{ }^{93} \mathrm{Zr},{ }^{93 \mathrm{~m}} \mathrm{Nb}$ and ${ }^{94} \mathrm{Nb}$ as activation products in fuel.

Any structure material and the shield in ITER are made of SS 316 with a significant amount of $M 0$ and some $T a$. Typical activation products comprise ${ }^{54} \mathrm{Mn},{ }^{55} \mathrm{Fe},{ }^{60} \mathrm{Co},{ }^{59} \mathrm{Ni},{ }^{63} \mathrm{Ni},{ }^{91} \mathrm{Nb},{ }^{93 m} \mathrm{Nb},{ }^{93} \mathrm{Mo}$ and ${ }^{99} \mathrm{Tc}$. The breeder blanket contains some ${ }^{10} \mathrm{Be},{ }^{39} \mathrm{Ar}$, and ${ }^{49} \mathrm{~V}$ in addition.

Any structure in RAFHT is made of RAF stainless steel and due to the high amount of $W$ some new radionuclides can be observed. The general activation products are ${ }^{53} \mathrm{Mn},{ }^{54} \mathrm{Mn},{ }^{55} \mathrm{Fe},{ }^{60} \mathrm{Co},{ }^{94} \mathrm{Nb},{ }^{108 m} \mathrm{Ag},{ }^{158} \mathrm{~Tb}$, ${ }^{179} \mathrm{Ta},{ }^{192} \mathrm{Ir}$ and ${ }^{210 \mathrm{~m}} \mathrm{Bi}$. The breeder blanket again has some ${ }^{39} \mathrm{Ar}$. The shield is made of $\mathrm{FeCrV}$ stainless steel and activation products for $\mathrm{FeCrV}$ are ${ }^{54} \mathrm{Mn},{ }^{55} \mathrm{Fe},{ }^{60} \mathrm{Co},{ }^{63} \mathrm{Ni},{ }^{83 m} \mathrm{Nb},{ }^{94} \mathrm{Nb},{ }^{93} \mathrm{Mo}$.

The bulk of the radionuclides is the same for all reactors and components and hence it is only the presence or absence of a couple of radionuclides and their activation that leads to a significantly higher or lower activation of the components. In the case of ITER and RAFHT, the difference certainly is due to the presence of ${ }^{59} \mathrm{Ni}$ and ${ }^{63} \mathrm{Ni}$ in $S S 316$ but their absence in $R A F$. The comparison of EBR-II and IFR teaches that ${ }^{83 m} \mathrm{Nb}$ and ${ }^{83} \mathrm{Mo}$ can be a substitute for the $N i$ isotopes in terms of resulting in a comparable activation. 


\section{Chapter 6}

\section{Summary and Conclusion}

\subsection{Summary}

A comparative study of radioactive waste from specific fast fission and specific fusion reactors has been conducted. First, radioactive waste from fast fission and fusion reactors was compared between two experimental nuclear reactors and between two commercial nuclear reactors. Second, radioactive waste from experimental and commercial nuclear reactors was compared between the two fast fission reactors and between the two fusion reactors.

The experimental and commercial nuclear reactors considered in this study were the Experimental Breeder Reactor II and the Integral Fast Reactor as the two fast fission reactors and the International Thermonuclear Experimental Reactor and the Reduced Activation Ferrite Helium Tokamak as the two fusion reactors.

The fast fission and fusion reactors were assumed to operate on a selfsufficient closed fuel cycle, i.e. they were assumed to breed the fuel needed for their operation. The importance of this assumption lies in the fact that radioactive waste from the two fast fission reactors did not contain actinides, nor did radioactive waste from the two fusion reactors contain gaseous or liquid tritium, because actinides and tritium as the respective bred fuels were recycled back to the reactor. Thereby, the fast fission reactors have the potential for fissioning long-lived actinides, i.e. transforming them into 
short-lived fission products. Thermal fission reactors do not support such a self-sufficient closed fuel cycle economically, because their breeding and actinide fissioning potentials are not high enough.

However, it must be noted that the separation of actinides or tritium from the remainder of the radioactive waste will not be complete, because the chemical processes involved do not allow for a $100 \%$ separation efficiency. Rather, typical separation efficiencies are in the $99.9 \%$ range. Thus the radioactive waste from the two specific fast fission reactors will always contain some actinides and that from the two specific fusion reactors will always contain some tritium. Consequently, the comparison conducted in this study represents an idealization in that it assumes $100 \%$ separation efficiency.

Recycling of structural material from the fusion reactors was not accounted for, because as of now, such recycling is a theoretical concept. On the other hand, recycling of actinides from the fast fission reactors has already been demonstrated in an experimental approach.

Radioactive waste from the four specific reactors was approximated as the solid fraction of reactor components. Reactor components were defined by their function within a reactor. In particular, the fuel, clad, duct, reflector and shield components of the fast fission reactors and the breeder blanket, first wall, duct or manifold and shield components of the fusion reactors were compared. Also compared was the sum of these components for the fast fission and the fusion reactors, as given in the tables and figures in Appendix B. Homogenization of the reactor components was necessary to increase the efficiency of this study.

The radioactive waste was characterized in terms of activation by radioactive waste parameters. Those parameters were calculated by computer codes. The central role for the fast fission reactors was played by ORIGEN$I I$, that for the fusion reactors by ONEDANT and REAC-II. All three codes were used to simulate build-up and decay of radionuclides in reactor com- 
ponents during and after irradiation by a neutron flux. Auxiliary computer codes were supplied to convert the output of those three computer codes into radioactive waste parameters.

The radioactive waste parameters chosen for this study were volume, radioactivity, whole body $\gamma$-dose rate, decay power, Biological Hazard Potential, radioactive waste class index and intruder dose rates. Those parameters were calculated for $100 s, 1 h, 1 d, 1 w, 1 y, 10 y, 100 y, 1,000 y$ and $10,000 y$ after discharge of the reactor components from the nuclear reactor. Furthermore, radioactive waste was assessed as cycle, life time and decommissioning radioactive waste.

Thereby, a short-, intermediate- and long-term characterization of radioactive waste was established. Thus, the radioactive waste parameters are applicable to waste handling as well as temporary or final waste storage. To our knowledge, no other study comprises this wide a spectrum of radioactive waste characterization.

\subsection{Conclusion}

The comparison conducted in Chapter 5 and the tables and figures presented in Appendix B suggest that, in the short- and intermediate-term after discharge, the specific activation of radioactive waste from the two fast fission reactors is higher than the specific activation of radioactive waste from the two fusion reactors. The specific activation of radioactive waste from the two commercial reactors is generally slightly higher than the specific activation of radioactive waste from the two experimental reactors.

However, in the long-term after discharge, a cross-over can be observed for the specific activation of radioactive waste from the fusion reactors but not for waste from the fast fission reactors. The same can be observed for the specific activation of radioactive waste from the experimental reactors but not for waste from the commercial reactors. Due to these cross-overs, a ranking list of specific activation of radioactive waste in the long-term would 
have IFR at its top, followed by ITER, EBR-II and RAFHT. However, the specific activation of $E B R-I I$ and $I T E R$ is not very different in the longterm.

On the other hand, in the short- and intermediate-term after discharge, the life-time absolute activation of radioactive waste from the experimental reactors is lower than that from the commercial reactors. Except for a very short time right after discharge, the life-time absolute activation of radioactive waste from the fast fission reactors is lower than that from the fusion reactors.

However, in the long-term after discharge, a cross-over can be observed for the life-time absolute activation of waste from the commercial reactors, but not for that from the experimental reactors. The same can be observed for the life-time absolute activation of radioactive waste from the fusion reactors, but not for that from the fast fission reactors. Due to these crossovers, a ranking list of life-time absolute activation of radioactive waste in the long-term would have ITER at its top, followed by IFR, RAFHT and $E B R-I I$. However, the life-time absolute activation of IFR and $R A F H T$ is not very different in the long-term. Note again, that $I F R$ is a $350 M W_{e}$ reactor, while $R A F H T$ generates $1,200 M W_{e}$, and $E B R-I I$ is rated at $60 M W_{t h}$, while ITER is rated at $1,000 M W_{t h}$.

The differences in the specifc and life-time absolute activation displayed by the waste of the four reference reactors, of course, are due to the different volumes of the reactor components. In particular, the fusion reactor components have larger volumes than the fission reactor components.

However, these conclusions have been drawn under the assumption that actinides are excluded from the radioactive waste of the fast fission reactors. Actinides increase the activation of radioactive waste from the fast fission reactors by a factor of less than two in the short- and intermediate-term, but by a factor of up to 1,000 in the long-term. If $100 \%$ of the actinides from the fast fission reactors are included in the radioactive waste, e.g. because a self-sufficient closed fuel cycle is not employed, then the comparison 
of the activation of radioactive waste from the fast fission and the fusion reactors and from the experimental and the commercial reactors will result in significantly different conclusions.

While the comparison of IFR and RAFHT would show an even increased gap in the long-term activation of the respective radioactive waste, the comparison of EBR-II and ITER presumably would show ITER as the experimental nuclear reactor with the lower long-term activation of radioactive waste, instead of $E B R-I I$ as in the case which excludes the actinides.

Considering that approximately $0.1 \%$ of the actinides may realistically remain in the radioactive waste from the two fast fission reactors, the longterm activation of the waste would roughly be two times, instead of thousand times, higher than that of the idealized actinide-free waste. This would not result in significantly different conclusions for the comparison.

$E B R$-II utilizes highly ${ }^{235} U$ enriched uranium fuel, while IFR utilizes a ${ }^{235} U$ depleted mixed uranium-plutonium-minor-actinides fuel. The longterm activation is determined by plutonium, and hence the long-term activation of radioactive waste from $I F R$ will always be higher than the long-term activation of radioactive waste from $E B R$ - $I I$, irrespective of the inclusion or exclusion of actinides.

The above conclusions have also been drawn under the assumption that the experimental fusion reactor utilizes standard materials, while the commercial fusion reactor utilizes Reduced Activation Materials (RAMs, such as Reduced Activation Ferrite). Assuming that the experimental fusion reactor utilizes $R A M \mathrm{~s}$ or the commercial fusion reactor utilizes standard materials can result in different conclusions.

If standard materials were utilized for the fusion reactors, the comparison of the two fast fission and the two fusion reactors would show not only ITER but also RAFHT with a higher long-term activation of the waste than that of $E B R-I I$ and $I F R$. This is supported by the three to six times higher intensity of irradiation, i.e. neutron flux, in $R A F H T$ with an only a factor of two shorter duration of irradiation as opposed to ITER. On the 
other hand, the comparison of the fast fission and the fusion reactors would show not only $R A F H T$ but also ITER with a lower long-term activation of the waste than that of $E B R$-II and IFR, if RAMs were utilized for the two fusion reactors. The decommissioning radioactive waste as presented in Table B.6 in Appendix B already features a higher absolute activation for the fusion reactors than for the fast fission reactors, regardless of the utilization of $R A M$ s.

Accordingly, the activation of radioactive waste from both the fast fission and the fusion reactors strongly depends on the materials used, although this dependence is much weaker for the fast fission reactors than for the fusion reactors. However, even utilization of $R A M \mathrm{~s}$ in the commercial fusion reactor can most likely not entirely bypass the production of high level radioactive waste and hence the need for provision of deep repositories would probably not be eliminated if the nuclear component of the energy supply structure switched from (fast) fission energy to fusion energy with fusion reactors of the analysed type. Only Low Activation Materials ( $L A M \mathrm{~s}$ ) [39], such as silicon carbide or vanadium-alloy, or the utilization of less neutrons producing fusion reactions, as the one between Deuterium and Helium ( $D-{ }_{2}^{3} H e$ reaction), can presumably eliminate this need.

Fusion reactor concepts, such as ARIES [20], based on these advanced materials and fuel cycles already exist. A comparison of the radioactive waste from such a reactor to that from IFR would certainly show, that the radioactive waste from IFR is far more hazardous than that from ARIES at all times after discharge. Yet it seems as if the development of both advanced materials and advanced fuel cycles does not receive the attention it should receive. This is especially important, if the fusion community is not to jeopardize one of it's major arguments for fusion: A significant reduction of radioactive waste compared to fission reactors.

In this study, the experimental reactors have the objective of establishing the foundation of commercial reactors. The latter are not conceivable 
without the former. Hence, the experimental and commercial reactors can be considered as a connected entity. This entity then has one investment and one return on investment. The investment in experimental reactors and the return on investment from commercial reactors have an economical and an ecological component. While the economical component is typically important in the short- and - at most - the intermediate-term, the ecological component is important in the long-term.

This study concentrated on the ecological component, which can be assessed in terms of the activation of radioactive waste produced by the experimental and commercial nuclear reactors. While the ecological investment is equivalent to the activation of radioactive waste from the experimental nuclear reactors, the ecological return on investment is equivalent to the difference in the activation of radioactive waste from the experimental and commercial nuclear reactors.

According to the above ranking of the nuclear reactors in the long-term, given the reactor designs in this study, the experimental fusion reactor requires a higher ecological investment than the experimental fast fission reactor. On the other hand, the commercial fusion reactor yields a positive ecological return on investment, while the commercial fast fission reactor yields a slightly negative one. This does not necessarily mean that eventually fusion reactors will be ecologically preferable to fission reactors, because a final evaluation of the ecological compatibility of fission and fusion reactor systems has to also account for all resources that are needed to operate those systems. 


\section{Bibliography}

[1] M.A. Abdou, C.C. Baker, M. Chazalon, P.J. Gierszewski, T. Kobayashi, K. Miya, J.E. Vetter, G. Casini, P. Dinner, A. Hishinuma, C. Holloway, D.F. Holland, C. Ponti, M.E. Sawan, D. Steiner, D.L. Smith, G. Vieider and H. Watanabe, "Summary of the ISFNT Workshop on the International Thermonuclear Experimental Reactor, Meeting Summary", International Symposium on Fusion Nuclear Technology, Tokyo, Japan, Fusion Technology, Volume 14, 1988, pp.1399-1414, November 1988.

[2] Argonne National Laboratory, "Technical Session Presentations, University of Chicago Review of the Engineering Physics Division", Argonne National Laboratory, Idaho Falls, Idaho, February 1989.

[3] T.L. Brown and H.E. LeMay, Jr., "Chemistry, The Central Science, Second Edition", Prentice-Hall Incorporation, Englewood Cliffs, New Jersey, ISBN 0-13-128504-1, 1981.

[4] L.J. Carter, "Nuclear Imperatives And Public Trust", Resources for the Future Incorporation, Washington, D.C., ISBN: 0-915707-29-2, 1987.

[5] Y.I. Chang, L.C. Walters, J.E. Battles, D.R. Pedersen, D.C. Wade and M.J. Lineberry, "Integral Fast Reactor Program Summary Progress Report FY1985 - FY1989", Argonne National Laboratory, Argonne, Ilinois, ANL-IFR-125, January 1990. 
[6] B.L. Cohen, "High Level Radioactive Waste from Light Water Reactors", University of Pittsburgh, Pittsburgh, Pennsylvania, Review of Modern Physics, Volume 49, 1, 1977, pp.1-20, January 1977.

[7] A.G. Croff, "User's Manual for the ORIGEN-II Computer Code", Oak Ridge National Laboratory, Oak Ridge, Tennessee, ORNL/TM7175/UC70, July 1980, latest supplementary Revision September 1989.

[8] S.A. Fetter, "Radiological Hazards of Fusion Reactors: Models and Comparisons", Dissertation, University of California, Berkeley, California, April 1985.

[9] D.F. Fischer and T.R. Johnson, "Immobilization of IFR Wastes in Mortar", SPECTRUM'88 ANS Fuel Cycle and Waste Management Division Meeting, Pasco, Washington, September 1988.

[10] C.H. Flanagan, W. Gulden, H. Iida, A. Kashirskij, B. Kolbasov, D. Leger, S. Moshkin, S. Piet, J. Raeder, Y. Seki, L. Topilskij and O. Tschipakin, "Safety and Environment for ITER, Records and Conclusions", ITER Specialists Meeting, Garching, Federal Republic of Germany, ITER-TN-SA-8-3, June 1988.

[11] Y. Gohar and H. Attaya, "US-Contributions to the Homework for ITER, Chapter III-a, Chapter IV", Argonne National Laboratory, Argonne, Illinois, March 1989.

[12] W. Gulden, C. Ponti, P. Guetat, D. Ochem, K. Broden, G. Olsson and G.J. Butterworth, "Fusion Waste Management - Safety and Environment Studies 1985 - 1986 -", Next European Torus, European Fusion Technology Programme, EUR-FU/XII-80/89-92, January 1989. 
[13] W. Häfele, J.P. Holdren, G. Kessler and G.L. Kulcinski, "Fusion and Fast Breeder Reactors", International Institute for Applied Systems Analysis, Laxenburg, Austria, RR-77-8, November 1976.

[14] R.N. Hill, "Private Communication", Argonne National Laboratory, Argonne, Mlinois June 1990 - April 1991.

[15] R.N. Hill, D.C. Wade, E.K. Fujita and H. Khalil, "Physics Studies of Higher Actinide Consumption in an LMR", Argonne National Laboratory, Argonne, Mlinois.

[16] R.N. Hill, "Physics Studies of Higher Actinide Consumption in an LMR, Intra-Laboratory Memo", Argonne National Laboratory, Argonne, Mlinois, September 1988.

[17] G.L. Hofman, A.G. Hins, D.L. Porter, L. Leibowitz and E.L. Wood, "Chemical Interaction of Metallic Fuel with Austenitic and Ferritic Stainless Steel Cladding", Argonne National Laboratory, Argonne, Illinois, International Conference on Reliable Fuels for Liquid Metal Reactors, Tucson, Arizona, September 1986.

[18] W.F. Holcomb, D.M. Paige, C.E. Stevenson and E.D. Graham, "High Level Radioactive Solid Waste Burial Operations For the EBR-II Fuel Cycle Facility", Argonne National Laboratory, Idaho Falls, Idaho, Nuclear Engineering and Design, 5, 1967, pp.221-227, January 1967.

[19] J.P. Holdren, D.H. Berwald, R.J. Budnitz, J.G. Crocker, J.G. Delene, R.D. Endicott, M.S. Kazimi, R.A. Krakowski, B.G. Logan and K.R. Schultz, "Report of the Senior Committee on Environmental, Safety, and Economic Aspects of Magnetic Fusion Energy", Laurence Livermore National Laboratory, Livermore, California, UCRL-59766, September 1989. 
[20] The ARIES Team, "The ARIES Tokamak Reactor Study", University of California, Los Angeles, California, UCLA-PPG-1274, October 1989.

[21] The ITER Team, "ITER Conceptual Design Interim Report", International Atomic Energy Agency, Vienna, Austria, October 1989.

[22] The ITER Team, "International Thermonuclear Experimental Reactor, ITER Concept Definition, Volume 2", International Atomic Energy Agency, Vienna, Austria, IAEA/ITER/DS/3, September 1989.

[23] The ITER Team, "International Thermonuclear Experimental Reactor, ITER Definition Phase", International Atomic Energy Agency, Vienna, Austria, IAEA/ITER/DS/2, July 1989.

[24] R.A. Johnson, S. Golan and E. Guenther, "SAFR, An Economic, Inherently Safe Liquid Metal Reactor", Rockwell International Corporation, Canoga Park, California, Nuclear Engineering and Design, 109, 1988, pp.87-95, April 1988.

[25] H. Khalil, E.K. Fujita, S. Yang and Y. Orchewa, "Fuel Management Studies of Small Metal and Oxide LMRs", Argonne National Laboratory, Argonne, Mlinois, Topical Meeting on Advances in Fuel Management, Pinehurst, North Carolina, March 1986.

[26] G.B. Krueger, C.E. Boardman, E.E. Olich, D.M. Switick, A.E Dubberley, F.J. Halfen and P.M. Magee, "PRISM: An Innovative Liquid Metal Fast Breeder Reactor", General Electric Company, Sunnyvale, California, Joint ASME/IEEE Power Generation Conference, Portland, Oregon, October 1986.

[27] J.R. Lamarsh, "Introduction to Nuclear Engineering, Second Edition", Addison-Wesley Publishing Company, Reading, Massachusetts, ISBN: 0-201-14200-7, 1983. 
[28] R.C. Maninger and D.W. Dorn, "Issues in Radioactive Waste Management for Fusion Power", Lawrence Livermore National Laboratory, Livermore, California, UCRL-87786, October 1982.

[29] F.M. Mann, "Private Communication", Hanford Engineering Development Laboratory, Richland, Washington, September 1989 - August 1990.

[30] F.M. Mann, "REAC-II: User's Manual and Code Description", Westinghouse Hanford Company, Richland, Washington, WHC-EP-0282, December 1989.

[31] J. Massidda, "Private Communication", Grumman Space Systems, Bethpage, New York, September 1989 - August 1990.

[32] R.D. O'Dell, F.W. Brinkley, Jr. and D.R. Marr, "User's Manual for ONEDANT: A Code Package for One-Dimensional Diffusion Accelerated, Neutral-Particle Transport", Los Alamos National Laboratory, Los Alamos, New Mexico, LA-9184-M, February 1982.

[33] OECD Nuclear Energy Agency and International Atomic Energy Agency, "Uranium Resources, Production and Demand - A Joint Report by the OECD-NEA and the IAEA",OECD-NEA and IAEA, Paris, France, 1990.

[34] Y. Orechwa and S.T. Yang, "Development of an LMR Core Design using Metallic Fuel", Argonne National Laboratory, Argonne, Illinois, International Conference on Reliable Fuel for Liquid Metal Reactors, Tucson, Arizona, September 1986.

[35] Y. Orchewa, H. Khalil, R.B. Turski and E.K. Fujita, "Core Design and Performance of Small Inherently Safe LMRs", Argonne National Laboratory, Argonne, Mlinois, Topical Meeting on Advances in Fuel Management, Pinehurst, North Carolina, March 1986. 
[36] B. Packard, C. Martell, "Private Communication", Arthur D. Little Incorporation, Cambridge, Massachusetts, February 1990.

[37] S. Piet, "Private Communication", Idaho National Engineering Laboratory, Idaho Falls, Idaho, September 1989 - August 1990.

[38] C. Ponti, "Special Materials for Fusion Reactors", European Communities' Joint Research Center, Ispra, Italy, July 1989.

[39] C. Ponti, "A New Trend in the Criteria for Low Activation Materials", European Communities' Joint Research Center, Ispra, Italy, July 1989.

[40] J. Raeder and S. Piet, "ITER Safety Analyses, Draft, Chapter 8", ITER Joint Work Session at the Max Planck Institut für Plasma Physik, Garching, Federal Republic of Germany, March 1990.

[41] J. Rifkin and T. Howard, "Entropy - A New World View", Bantam Books, New York, New York, ISBN: 0-553-20215-4, 1981.

[42] L.N. Salerno, R.C. Berglund, G.L. Gyorey, F.E. Tippets and P.M. Tschamper, "PRISM Concept, Modular LMR Reactors", General Electric Company, San Jose, California, Nuclear Engineering and Design, 109, 1988, pp.79-86, April 1988.

[43] D.L. Smith, C.C. Baker, D.K. Sze, G.D. Morgan, M.A. Abdou, S.J. Piet, K.R. Schultz, R.W. Moir and J.D. Gordon, "Overview of the Blanket Comparison and Selection Study", Fusion Technology, Volume 8, 10, 1985, October 1985 .

[44] C.E. Stevenson, "The EBR-II Fuel Cycle Story", American Nuclear Society, La Grange Park, Illinois, ISBN: 0-89448-031-6, 1987. 
[45] C.E. Till, "The US Advanced Reactor Development Program", Argonne National Laboratory, Argonne, Illinois, International Reactor Physics Conference, Jackson, Wyoming, September 1988.

[46] C.E. Till and Y.I. Chang, "The Integral Fast Reactor Concept", Argonne National Laboratory, Argonne, Mlinois, 48th Annual Meeting of the American Power Conference, Chicago, Illinois, April 1986.

[47] United States Nuclear Regulatory Commission, "Rules and Regulations, Standards for Protection against Radiation", Title 10, Chapter 1, Code of Federal Regulations - Energy, Part 20, 10CFR20, January 1990.

[48] United States Nuclear Regulatory Commission, "Rules and Regulations, Disposal of High Level Radioactive Waste in Geological Repositories", Title 10, Chapter 1, Code of Federal Regulations - Energy, Part 60, 10CFR60, January 1990.

[49] United States Nuclear Regulatory Commission, "Rules and Regulations, Licensing Requirements for Land Disposal of Radioactive Waste", Title 10, Chapter 1, Code of Federal Regulations - Energy, Part 61, 10CFR61, January 1990.

[50] S. Vogler, J. Jung, M.J. Steindler, I. Maya, H.E. Levine, D.D. Peterman, S. Strausburg and K.R. Schultz, "Materials Recycle and Waste Management in Fusion Power Reactors", Progress Report for 1982, Argonne National Laboratory, Argonne, Ilinois, ANL/FPP/TM-163, January 1983.

[51] D.C. Wade and Y.I. Chang, "The Integral Fast Reactor Concept: Physics of Operation and Safety", Argonne National Laboratoty, Argonne, Mlinois, Nuclear Science and Engineering, 100, 1988, pp.507524, December 1988. 
[52] F.W. Walker, J.R. Parrington and F. Feiner, "Nuclides and Isotopes, Fourteenth Edition, Chart of the Nuclides", General Electric Company Nuclear Energy Operations, San Jose,California, 1989.

[53] A.E. Waltar and A.B. Reynolds, "Fast Breeder Reactors", Pergamon Press, 1981.

[54] L.C. Walters, B.R. Seidel and J.H. Kittel, "Performance of Metallic Fuels and Blankets in Liquid Metal Fast Breeder Reactors", Argonne National Laboratory, Argonne, Ilinois, Nuclear Technology, 65, 1984, pp.179-231, May 1984.

[55] L.C. Witbeck and R.M. Fryer, "Waste Production and Management at EBR-II", Argonne National Laboratory, Argonne, Mlinois, UC-79, April 1979.

[56] A. Ziegler, "Lehrbuch der Reaktortechnik, Band 2: Reaktortechnik", Springer-Verlag, Berlin, Federal Republic of Germany, ISBN: 3-54013180-9, April 1984. 


\section{Appendix A}

\section{Reactor Materials and Geometries}

Tables A.1, A.2, A.3 and A show the isotope and element composition of the Experimental Breeder Reactor II components. Tables A.5, A.6, A.7 and A.8 show the isotope and element composition of the Integral Fast Reactor components.

Table A.9 shows the material composition of the International Thermonuclear Experimental Reactor components. Tables A.10, A.11, A.12 and A.13 show the radial build of the materials of ITER at the reactor midplane.

Table A.14 shows the material composition of the Reduced Activation Ferrite Helium Tokamak components. Tables A.15 and A.16 show the radial build of the materials of RAFHT at the reactor midplane.

Finally, Tables A.17 and A.18 show the element composition including impurities of some materials of the four reactors. 


\begin{tabular}{|l|r|r|r|}
\hline \hline Element & Driver & $\begin{array}{r}\text { Internal } \\
\text { Breeder }\end{array}$ & $\begin{array}{r}\text { Radial } \\
\text { Breeder }\end{array}$ \\
\cline { 2 - 4 } & {$[\mathrm{kg}]$} & {$[\mathrm{kg}]$} & {$[\mathrm{kg}]$} \\
\hline$m_{C}$ & $2.99084 \cdot 10^{-4}$ & $3.82444 \cdot 10^{-4}$ & $4.99785 \cdot 10^{-4}$ \\
$m_{S i}$ & $9.96947 \cdot 10^{-3}$ & $1.27481 \cdot 10^{-2}$ & $1.66595 \cdot 10^{-2}$ \\
$m_{P}$ & $4.48626 \cdot 10^{-4}$ & $5.73666 \cdot 10^{-4}$ & $7.49677 \cdot 10^{-4}$ \\
$m_{S}$ & $2.99084 \cdot 10^{-4}$ & $3.82444 \cdot 10^{-4}$ & $4.99785 \cdot 10^{-4}$ \\
$m_{C r}$ & $1.89420 \cdot 10^{-1}$ & $2.42214 \cdot 10^{-1}$ & $3.16530 \cdot 10^{-1}$ \\
$m_{M n}$ & $1.99389 \cdot 10^{-2}$ & $2.54963 \cdot 10^{-2}$ & $3.33190 \cdot 10^{-2}$ \\
$m_{F e}$ & $6.76877 \cdot 10^{-1}$ & $8.65534 \cdot 10^{-1}$ & $1.13110 \cdot 10^{+0}$ \\
$m_{N i}$ & $9.96947 \cdot 10^{-2}$ & $1.27481 \cdot 10^{-1}$ & $1.66595 \cdot 10^{-1}$ \\
\hline$\sum_{e=1}^{n_{\bullet}} m_{e}$ & $9.96947 \cdot 10^{-1}$ & $1.27481 \cdot 10^{+0}$ & $1.66595 \cdot 10^{+0}$ \\
\hline \hline
\end{tabular}

Table A.1: Element Composition of Assembly of Experimental Breeder Reactor II Clad Component

\begin{tabular}{|l|r|r|r|}
\hline \hline Element & Driver & $\begin{array}{r}\text { Internal } \\
\text { Breeder }\end{array}$ & $\begin{array}{r}\text { Radial } \\
\text { Breeder }\end{array}$ \\
\cline { 2 - 4 } & {$[\mathrm{kg}]$} & {$[\mathrm{kg}]$} & {$[\mathrm{kg}]$} \\
\hline$m_{C}$ & $2.12303 \cdot 10^{-4}$ & $8.59607 \cdot 10^{-4}$ & $1.07191 \cdot 10^{-3}$ \\
$m_{S i}$ & $7.07676 \cdot 10^{-3}$ & $2.86535 \cdot 10^{-2}$ & $3.57303 \cdot 10^{-2}$ \\
$m_{P}$ & $3.18454 \cdot 10^{-4}$ & $1.28941 \cdot 10^{-3}$ & $1.60786 \cdot 10^{-3}$ \\
$m_{S}$ & $2.12303 \cdot 10^{-4}$ & $8.59607 \cdot 10^{-4}$ & $1.07191 \cdot 10^{-3}$ \\
$m_{C r}$ & $1.34458 \cdot 10^{-1}$ & $5.44417 \cdot 10^{-1}$ & $6.78875 \cdot 10^{-1}$ \\
$m_{M n}$ & $1.41535 \cdot 10^{-2}$ & $5.73070 \cdot 10^{-2}$ & $7.14605 \cdot 10^{-2}$ \\
$m_{F e}$ & $4.80477 \cdot 10^{-1}$ & $1.94543 \cdot 10^{+0}$ & $2.42591 \cdot 10^{+0}$ \\
$m_{N i}$ & $7.07676 \cdot 10^{-2}$ & $2.86535 \cdot 10^{-1}$ & $3.57303 \cdot 10^{-1}$ \\
\hline$\sum_{e=1}^{n_{e}} m_{e}$ & $7.07673 \cdot 10^{-1}$ & $2.86535 \cdot 10^{+0}$ & $3.57303 \cdot 10^{+0}$ \\
\hline \hline
\end{tabular}

Table A.2: Element Composition of Assembly of Experimental Breeder Reactor II Duct Component 


\begin{tabular}{|c|c|c|c|}
\hline \multirow[t]{2}{*}{$\begin{array}{l}\text { Isotope or } \\
\text { Element }\end{array}$} & $\overline{\text { Driver }}$ & $\begin{array}{l}\text { Internal } \\
\text { Breeder }\end{array}$ & $\begin{array}{r}\text { Radial } \\
\text { Breeder }\end{array}$ \\
\hline & {$[\mathrm{kg}]$} & {$[k g]$} & {$[\mathrm{kg}]$} \\
\hline$m_{23 \mathrm{~B}} U$ & $2.50014 \cdot 10^{+0}$ & $1.13588 \cdot 10^{-1}$ & $1.48439 \cdot 10^{-1}$ \\
\hline$m_{23} s_{0 u}$ & $4.40749 \cdot 10^{-3}$ & 0.0 & 0.0 \\
\hline$m_{241} P_{u}$ & $1.61348 \cdot 10^{-4}$ & 0.0 & 0.0 \\
\hline$\sum_{i=1}^{n_{i}} m_{i}$ & $2.50471 \cdot 10^{+0}$ & $1.13588 \cdot 10^{-1}$ & $1.48439 \cdot 10^{-1}$ \\
\hline$m 234 U$ & $8.84432 \cdot 10^{-3}$ & $8.63996 \cdot 10^{-4}$ & $1.12909 \cdot 10^{-3}$ \\
\hline$m_{236} U$ & $3.56656 \cdot 10^{-2}$ & 0.0 & 0.0 \\
\hline$m_{238} U$ & $2.25028 \cdot 10^{+0}$ & $1.58616 \cdot 10^{+1}$ & $2.07283 \cdot 10^{+1}$ \\
\hline$m_{236} P_{u}$ & $0.18495 \cdot 10^{-9}$ & 0.0 & 0.0 \\
\hline$m_{238} P u$ & $2.37723 \cdot 10^{-5}$ & 0.0 & 0.0 \\
\hline$m_{240} P u$ & $1.40307 \cdot 10^{-3}$ & 0.0 & 0.0 \\
\hline$m_{242} P_{u}$ & $7.38460 \cdot 10^{-5}$ & 0.0 & 0.0 \\
\hline$\sum_{i=1}^{n_{i}} m_{i}$ & $4.80100 \cdot 10^{+0}$ & $1.59761 \cdot 10^{+1}$ & $2.08779 \cdot 10^{+1}$ \\
\hline$m_{S i}$ & $3.54056 \cdot 10^{-3}$ & 0.0 & 0.0 \\
\hline$m_{Z r}$ & $4.55215 \cdot 10^{-3}$ & 0.0 & 0.0 \\
\hline$m_{N b}$ & $5.05794 \cdot 10^{-4}$ & 0.0 & 0.0 \\
\hline$m_{M 0}$ & $1.26449 \cdot 10^{-1}$ & 0.0 & 0.0 \\
\hline$m_{T_{c}}$ & $2.52897 \cdot 10^{-3}$ & 0.0 & 0.0 \\
\hline$m_{R u}$ & $9.71125 \cdot 10^{-2}$ & 0.0 & 0.0 \\
\hline$m_{R h}$ & $1.31507 \cdot 10^{-2}$ & 0.0 & 0.0 \\
\hline$m_{P d}$ & $9.10430 \cdot 10^{-3}$ & 0.0 & 0.0 \\
\hline$\sum_{i e=1}^{n_{i e}} m_{i e}$ & $5.05794 \cdot 10^{+0}$ & $1.59761 \cdot 10^{+1}$ & $2.08779 \cdot 10^{+1}$ \\
\hline$\xi_{U}$ & 0.94800 & 1.0 & 1.0 \\
\hline$\xi_{P_{u}}$ & 0.00120 & 0.0 & 0.0 \\
\hline$\xi_{S i}$ & 0.00070 & 0.0 & 0.0 \\
\hline$\xi_{Z r}$ & 0.00090 & 0.0 & 0.0 \\
\hline$\xi_{N b}$ & 0.00010 & 0.0 & 0.0 \\
\hline$\xi_{M 0}$ & 0.02500 & 0.0 & 0.0 \\
\hline$\xi_{T c}$ & 0.00050 & 0.0 & 0.0 \\
\hline$\xi_{R u}$ & 0.01920 & 0.0 & 0.0 \\
\hline$\xi_{R h}$ & 0.00260 & 0.0 & 0.0 \\
\hline$\xi_{P d}$ & 0.00180 & 0.0 & 0.0 \\
\hline
\end{tabular}

Table A.3: Isotope and Element Composition of Assembly of Experimental Breeder Reactor II Fuel Component, Adapted from [44] 


\begin{tabular}{|l|r|}
\hline \hline Element & Shield \\
\cline { 2 - 2 } & {$[\mathrm{kg}]$} \\
\hline$m_{C}$ & $2.52512 \cdot 10^{+1}$ \\
$m_{S i}$ & $8.41706 \cdot 10^{+2}$ \\
$m_{P}$ & $3.78768 \cdot 10^{+1}$ \\
$m_{S}$ & $2.52512 \cdot 10^{+1}$ \\
$m_{C r}$ & $1.59924 \cdot 10^{+3}$ \\
$m_{M n}$ & $1.68341 \cdot 10^{+2}$ \\
$m_{F_{e}}$ & $5.46225 \cdot 10^{+3}$ \\
$m_{N i}$ & $8.41706 \cdot 10^{+2}$ \\
\hline$m_{=\sum_{e=1}^{n_{e}} m_{e}}$ & $8.41706 \cdot 10^{+3}$ \\
\hline \hline
\end{tabular}

Table A.4: Element Composition of $100 \%$ of Experimental Breeder Reactor II Shield Component

\begin{tabular}{|l|r|r|r|}
\hline \hline Element & Driver & $\begin{array}{r}\text { Internal } \\
\text { Breeder }\end{array}$ & $\begin{array}{r}\text { Radial } \\
\text { Breeder }\end{array}$ \\
\cline { 2 - 4 } & $1.12304 \cdot 10^{+1}$ & $3.74425 \cdot 10^{+0}$ & $4.85740 \cdot 10^{+0}$ \\
\hline$m_{C}$ & $1.97870 \cdot 10^{-1}$ & $6.59700 \cdot 10^{-2}$ & $8.55800 \cdot 10^{-2}$ \\
$m_{N}$ & $3.10170 \cdot 10^{-1}$ & $1.03410 \cdot 10^{-1}$ & $1.34160 \cdot 10^{-1}$ \\
$m_{O}$ & $1.17652 \cdot 10^{+1}$ & $3.92254 \cdot 10^{+0}$ & $5.08871 \cdot 10^{+0}$ \\
$m_{S i}$ & $1.06960 \cdot 10^{-1}$ & $3.56600 \cdot 10^{-2}$ & $4.62600 \cdot 10^{-2}$ \\
$m_{T i}$ & $1.81826 \cdot 10^{+1}$ & $6.06211 \cdot 10^{+0}$ & $7.86436 \cdot 10^{+0}$ \\
$m_{V}$ & $6.42808 \cdot 10^{+2}$ & $2.14314 \cdot 10^{+2}$ & $2.78028 \cdot 10^{+2}$ \\
$m_{C r}$ & $2.67391 \cdot 10^{+1}$ & $8.91487 \cdot 10^{+0}$ & $1.15652 \cdot 10^{+1}$ \\
$m_{M n}$ & $4.52364 \cdot 10^{+3}$ & $1.50819 \cdot 10^{+3}$ & $1.95657 \cdot 10^{+3}$ \\
$m_{F_{e}}$ & $3.04826 \cdot 10^{+1}$ & $1.01630 \cdot 10^{+1}$ & $1.31844 \cdot 10^{+1}$ \\
$m_{N i}$ & $5.50826 \cdot 10^{+1}$ & $1.83646 \cdot 10^{+1}$ & $2.38244 \cdot 10^{+1}$ \\
$m_{M o}$ & $2.72739 \cdot 10^{+1}$ & $9.09317 \cdot 10^{+0}$ & $1.17966 \cdot 10^{+1}$ \\
$m_{W}$ & $5.34782 \cdot 10^{+3}$ & $1.78298 \cdot 10^{+3}$ & $2.31305 \cdot 10^{+3}$ \\
\hline$\sum_{e=1}^{n_{e} m_{e}}$ & 5
\end{tabular}

Table A.5: Element Composition of $100 \%$ of Integral Fast Reactor Clad Component 


\begin{tabular}{|l|r|r|r|}
\hline \hline Element & Driver & $\begin{array}{r}\text { Internal } \\
\text { Breeder }\end{array}$ & $\begin{array}{r}\text { Radial } \\
\text { Breeder }\end{array}$ \\
\cline { 2 - 4 } & {$[\mathrm{kg}]$} & {$[\mathrm{kg}]$} & {$[\mathrm{kg}]$} \\
\hline$m_{C}$ & $6.53549 \cdot 10^{+0}$ & $2.48361 \cdot 10^{+0}$ & $3.22198 \cdot 10^{+0}$ \\
$m_{N}$ & $1.15150 \cdot 10^{-1}$ & $4.37600 \cdot 10^{-2}$ & $5.67700 \cdot 10^{-2}$ \\
$m_{O}$ & $1.80500 \cdot 10^{-1}$ & $6.85900 \cdot 10^{-2}$ & $8.89900 \cdot 10^{-2}$ \\
$m_{S i}$ & $6.84670 \cdot 10^{+0}$ & $2.60188 \cdot 10^{+0}$ & $3.37541 \cdot 10^{+0}$ \\
$m_{T_{i}}$ & $6.22400 \cdot 10^{-2}$ & $2.36500 \cdot 10^{-2}$ & $3.06900 \cdot 10^{-2}$ \\
$m_{V}$ & $1.05813 \cdot 10^{+1}$ & $4.02108 \cdot 10^{+0}$ & $5.21654 \cdot 10^{+0}$ \\
$m_{C r}$ & $3.74079 \cdot 10^{+2}$ & $1.42157 \cdot 10^{+2}$ & $1.84420 \cdot 10^{+2}$ \\
$m_{M n}$ & $1.55607 \cdot 10^{+1}$ & $5.91335 \cdot 10^{+0}$ & $7.67138 \cdot 10^{+0}$ \\
$m_{F e}$ & $2.63251 \cdot 10^{+3}$ & $1.00040 \cdot 10^{+3}$ & $1.29782 \cdot 10^{+3}$ \\
$m_{N i}$ & $1.77392 \cdot 10^{+1}$ & $6.74122 \cdot 10^{+0}$ & $8.74537 \cdot 10^{+0}$ \\
$m_{M o}$ & $3.20550 \cdot 10^{+1}$ & $1.21815 \cdot 10^{+1}$ & $1.58030 \cdot 10^{+1}$ \\
$m_{W}$ & $1.58719 \cdot 10^{+1}$ & $6.03162 \cdot 10^{+0}$ & $7.82480 \cdot 10^{+0}$ \\
\hline$\sum_{e=1}^{n_{e}} m_{e}$ & $3.11214 \cdot 10^{+3}$ & $1.18267 \cdot 10^{+3}$ & $1.53428 \cdot 10^{+3}$ \\
\hline \hline
\end{tabular}

Table A.6: Element Composition of $100 \%$ of Integral Fast Reactor Duct Component 


\begin{tabular}{|c|c|c|c|}
\hline \multirow[t]{2}{*}{$\begin{array}{l}\text { Isotope or } \\
\text { Element }\end{array}$} & Driver & $\begin{array}{l}\text { Internal } \\
\text { Breeder }\end{array}$ & $\begin{array}{r}\text { Radial } \\
\text { Breeder }\end{array}$ \\
\hline & {$[\mathrm{kg}]$} & {$[\mathrm{kg}]$} & {$[\mathrm{kg}]$} \\
\hline$m_{236} U$ & $2.83780 \cdot 10^{+0}$ & $2.27500 \cdot 10^{+0}$ & $2.95000 \cdot 10^{+0}$ \\
\hline$m_{23{ }_{P} \mathrm{Pu}}$ & $3.28210 \cdot 10^{+2}$ & 0.0 & 0.0 \\
\hline$m_{241} P_{u}$ & $1.20086 \cdot 10^{+1}$ & 0.0 & 0.0 \\
\hline$\sum_{i=1}^{n_{i}} m_{i}$ & $3.43056 \cdot 10^{+2}$ & $2.27500 \cdot 10^{+0}$ & $2.95000 \cdot 10^{+0}$ \\
\hline$m_{234} \mathrm{U}$ & $5.62441 \cdot 10^{-3}$ & 0.0 & 0.0 \\
\hline$m_{230_{U}}$ & $2.26810 \cdot 10^{-2}$ & 0.0 & 0.0 \\
\hline$m_{298 U}$ & $1.51099 \cdot 10^{+3}$ & $1.13680 \cdot 10^{+3}$ & $1.47478 \cdot 10^{+3}$ \\
\hline$m_{237} N_{p}$ & $2.19278 \cdot 10^{+0}$ & 0.0 & 0.0 \\
\hline$m_{236} P_{u}$ & $1.37720 \cdot 10^{-5}$ & 0.0 & 0.0 \\
\hline$m_{238} P_{u}$ & $1.78024 \cdot 10^{+0}$ & 0.0 & 0.0 \\
\hline$m_{240} P u$ & $1.04481 \cdot 10^{+2}$ & 0.0 & 0.0 \\
\hline$m_{242} P_{u}$ & $5.48666 \cdot 10^{+0}$ & 0.0 & 0.0 \\
\hline$m_{241} A m$ & $2.28644 \cdot 10^{-1}$ & 0.0 & 0.0 \\
\hline$m_{242} A m$ & 0.0 & 0.0 & 0.0 \\
\hline$m_{24 s_{A m}}$ & 0.0 & 0.0 & 0.0 \\
\hline$m_{242} \mathrm{Cm}$ & $6.46740 \cdot 10^{-3}$ & 0.0 & 0.0 \\
\hline$m_{243} \mathrm{Cm}$ & $2.87062 \cdot 10^{-3}$ & 0.0 & 0.0 \\
\hline$m_{244} \mathrm{Cm}$ & $1.24909 \cdot 10^{-1}$ & 0.0 & 0.0 \\
\hline$m_{245} \mathrm{Cm}$ & $2.69690 \cdot 10^{-2}$ & 0.0 & 0.0 \\
\hline$m_{240} \mathrm{Cm}$ & $9.88236 \cdot 10^{-3}$ & 0.0 & 0.0 \\
\hline$\sum_{i=1}^{n_{i}} m_{i}$ & $1.97098 \cdot 10^{+3}$ & $1.13908 \cdot 10^{+3}$ & $1.47773 \cdot 10^{+3}$ \\
\hline$m_{z r}$ & $2.18997 \cdot 10^{+2}$ & $1.26564 \cdot 10^{+2}$ & $1.64192 \cdot 10^{+2}$ \\
\hline$m_{\text {Balan }}$ & $2.56148 \cdot 10^{+0}$ & 0.0 & 0.0 \\
\hline$\sum_{i e=1}^{n_{i e}} m_{i e}$ & $2.18997 \cdot 10^{+3}$ & $1.26564 \cdot 10^{+3}$ & $1.64192 \cdot 10^{+3}$ \\
\hline$\xi_{U}$ & 0.69127 & 0.9 & 0.9 \\
\hline$\xi_{P u}$ & 0.20638 & 0.0 & 0.0 \\
\hline$\xi_{A m}$ & 0.00010 & 0.0 & 0.0 \\
\hline$\xi_{C m}$ & 0.00008 & 0.0 & 0.0 \\
\hline$\xi_{Z r}$ & 0.10000 & 0.1 & 0.1 \\
\hline
\end{tabular}

Table A.7: Isotope and Element Composition of $25 \%$ of Integral Fast Reactor Fuel Component, Adapted from [51] 


\begin{tabular}{|l|r|r|}
\hline \hline \multirow{2}{*}{ Element } & Reflector & Shield \\
\cline { 2 - 3 } & {$[\mathrm{kg}]$} & {$[\mathrm{kg}]$} \\
\hline$m_{C}$ & $3.12204 \cdot 10^{+1}$ & $1.95510 \cdot 10^{+1}$ \\
$m_{N}$ & $5.50070 \cdot 10^{-1}$ & $3.44470 \cdot 10^{-1}$ \\
$m_{O}$ & $8.62280 \cdot 10^{-1}$ & $5.39980 \cdot 10^{-1}$ \\
$m_{S i}$ & $3.27071 \cdot 10^{+1}$ & $2.04820 \cdot 10^{+1}$ \\
$m_{T i}$ & $2.97340 \cdot 10^{-1}$ & $1.86200 \cdot 10^{-1}$ \\
$m_{V}$ & $5.05473 \cdot 10^{+1}$ & $3.16540 \cdot 10^{+1}$ \\
$m_{C r}$ & $1.78700 \cdot 10^{+3}$ & $1.11906 \cdot 10^{+3}$ \\
$m_{M n}$ & $7.43343 \cdot 10^{+1}$ & $4.65500 \cdot 10^{+1}$ \\
$m_{F e}$ & $1.25756 \cdot 10^{+4}$ & $7.87519 \cdot 10^{+3}$ \\
$m_{N i}$ & $8.47410 \cdot 10^{+1}$ & $5.30670 \cdot 10^{+1}$ \\
$m_{M o}$ & $1.53129 \cdot 10^{+2}$ & $9.58930 \cdot 10^{+1}$ \\
$m_{W}$ & $7.58209 \cdot 10^{+1}$ & $4.74810 \cdot 10^{+1}$ \\
\hline$\sum_{e=1}^{n_{e}} m_{e}$ & $1.48668 \cdot 10^{+4}$ & $9.30999 \cdot 10^{+3}$ \\
\hline$m_{B}$ & 0.0 & $3.68987 \cdot 10^{+3}$ \\
$m_{C}$ & 0.0 & $1.02486 \cdot 10^{+3}$ \\
\hline$\sum_{e=1}^{n_{e}} m_{e}$ & $1.48668 \cdot 10^{+4}$ & $1.40247 \cdot 10^{+4}$ \\
\hline \hline
\end{tabular}

Table A.8: Element Composition of $100 \%$ of Integral Fast Reactor Reflector and Shield Components 


\begin{tabular}{|c|c|c|c|c|}
\hline Component & Material & $\begin{array}{r}\text { Inboard } \\
\text { [volume- } \% \text { ] }\end{array}$ & $\begin{array}{l}\text { Outboard } \\
\text { [volume-\%] }\end{array}$ & $\begin{array}{r}\text { Total } \\
\text { volume- } \% \text { ] }\end{array}$ \\
\hline Protective Layer & $\bar{C}$ & 100.0 & - & 100.0 \\
\hline First Wall & $S S 316$ & 100.0 & 100.0 & 100.0 \\
\hline \multirow[t]{4}{*}{ Breeder Blanket } & $S S 316$ & $\overline{4.4}$ & 3.2 & 3.3 \\
\hline & $\mathrm{Li}_{2} \mathrm{O}$ & 6.7 & 4.8 & 5.1 \\
\hline & $\mathrm{H}_{2} \mathrm{O}$ & 3.7 & 1.8 & 2.1 \\
\hline & $B e$ & 85.2 & 90.2 & 89.5 \\
\hline Back Wall & $S S 316$ & 100.0 & 100.0 & 100.0 \\
\hline \multirow[t]{2}{*}{ Steel Shield } & $S S 316$ & 65.7 & $\begin{array}{ll}68.8 \\
\end{array}$ & 68.5 \\
\hline & $\mathrm{H}_{2} \mathrm{O}$ & 34.3 & 31.2 & 31.5 \\
\hline \multirow[t]{2}{*}{ Lead Shield } & $\overline{P b}$ & 70.0 & 70.0 & 70.0 \\
\hline & $\mathrm{B}_{4} \mathrm{C}$ & 30.0 & 30.0 & 30.0 \\
\hline Vacuum Vessel & $S S 316$ & 100.0 & 100.0 & 100.0 \\
\hline \multirow[t]{5}{*}{ Toroidal Coils } & $S S 316$ & 43.0 & 43.0 & 43.0 \\
\hline & $\mathrm{Nb}_{3} \mathrm{Sn}$ & 8.7 & 8.7 & 8.7 \\
\hline & $C u$ & 20.3 & 20.3 & 20.3 \\
\hline & Mylar & 8.0 & 8.0 & 8.0 \\
\hline & $\mathrm{He}$ & 20.0 & 20.0 & 20.0 \\
\hline \multirow[t]{5}{*}{ Poloidal Coil } & $S S 316$ & 43.0 & - & 43.0 \\
\hline & $\mathrm{Nb}_{3} \mathrm{Sn}$ & 8.7 & - & 8.7 \\
\hline & $\mathrm{Cu}$ & 20.3 & - & 20.3 \\
\hline & Mylar & 8.0 & - & 8.0 \\
\hline & $\mathrm{H}_{2} \mathrm{O}$ & 20.0 & - & 20.0 \\
\hline \multirow[t]{11}{*}{ Reactor } & $S S 316$ & 54.3 & 56.8 & 56.1 \\
\hline & $\overline{P b}$ & 1.3 & 1.3 & 1.2 \\
\hline & $\mathrm{Nb}_{3} \mathrm{Sn}$ & 4.3 & 1.4 & 2.0 \\
\hline & $C u$ & 10.0 & 3.3 & 4.8 \\
\hline & $\mathrm{B}_{4} \mathrm{C}$ & 0.6 & 0.5 & 0.4 \\
\hline & $\mathrm{Li}_{2} \mathrm{O}$ & 0.6 & 1.0 & 0.8 \\
\hline & $\mathrm{H}_{2} \mathrm{O}$ & 9.8 & 13.0 & 12.2 \\
\hline & Mylar & 3.9 & 1.3 & 1.8 \\
\hline & $C$ & 1.4 & - & 1.3 \\
\hline & $\overline{B e}$ & 8.2 & 18.2 & 15.7 \\
\hline & $\mathrm{He}$ & 5.6 & 3.2 & 3.7 \\
\hline
\end{tabular}

Table A.9: Material Composition of International Thermonuclear Experimental Reactor Components, Adapted from [11,21,22] 


\begin{tabular}{|l|r|r|r|r|r|}
\hline \hline Component & \multirow{2}{*}{ Material } & $\mathbf{R}_{\mathbf{m c}}$ & $\mathbf{R}_{\mathbf{m c}_{\mathbf{o}}}$ & $\mathbf{R}_{\mathbf{m c}_{\mathbf{o}}}-\mathbf{R}_{\mathbf{m c}}$ & $\rho_{\mathbf{m}}$ \\
\cline { 3 - 6 } & & {$[\mathrm{mm}]$} & {$[\mathrm{mm}]$} & {$[\mathrm{mm}]$} & {$\left[\frac{t}{\mathbf{m}^{3}}\right]$} \\
\hline Protective Layer & $\mathrm{C}$ & 3680 & 3700 & 20 & 2.25 \\
\hline First Wall & $S S 316$ & 3675 & 3680 & 5 & 7.90 \\
\hline Breeder Blanket & $\mathrm{H}_{\mathbf{2}} \mathrm{O}$ & 3672 & 3675 & 3 & 1.00 \\
& $S S 316$ & 3670 & 3672 & 2 & 7.90 \\
& $B e$ & 3615 & 3670 & 55 & 1.85 \\
& $S S 316$ & 3614 & 3615 & 1 & 7.90 \\
& $\mathrm{Li}_{\mathbf{2}} \mathrm{O}$ & 3605 & 3614 & 9 & 2.01 \\
& $S S 316$ & 3604 & 3605 & 1 & 7.90 \\
& $B e$ & 3544 & 3604 & 60 & 1.85 \\
& $S S 316$ & 3542 & 3544 & 2 & 7.90 \\
& $\mathrm{H}_{2} \mathrm{O}$ & 3540 & 3542 & 2 & 1.00 \\
\hline Back Wall & $S S 316$ & 3470 & 3540 & 70 & 7.90 \\
\hline Gap & Vacuum & 3450 & 3470 & 20 & 0.00 \\
\hline \hline
\end{tabular}

Table A.10: Radial Build of International Thermonuclear Experimental Reactor Inboard Components, Adapted from [11,21] 


\begin{tabular}{|c|c|c|c|c|c|}
\hline \multirow[t]{2}{*}{ Component } & \multirow[t]{2}{*}{ Material } & $\mathbf{R}_{\mathbf{m} \mathbf{c}_{\mathrm{i}}}$ & $\mathbf{R}_{\mathbf{m c o}_{\mathrm{o}}}$ & $\overline{\mathbf{R}_{\mathbf{m c o}_{0}}-\mathbf{R}_{\mathbf{m c}_{i}}}$ & $\rho_{\mathbf{m}}$ \\
\hline & & {$[\mathrm{mm}]$} & {$[\mathrm{mm}]$} & {$[\mathrm{mm}]$} & {$\left[\frac{t}{m^{3}}\right]$} \\
\hline \multirow[t]{5}{*}{ Steel Shield } & $\overline{S S 316}$ & 3400 & 3450 & 50 & 7.90 \\
\hline & $\mathrm{H}_{2} \mathrm{O}$ & 3390 & 3400 & 10 & 1.00 \\
\hline & $S S 316$ & 3310 & 3390 & 80 & 7.90 \\
\hline & $\mathrm{H}_{2} \mathrm{O}$ & 3240 & 3310 & 70 & 1.00 \\
\hline & $S S 316$ & 3220 & 3240 & 20 & 7.90 \\
\hline Lead Shield & $\mathrm{Pb} / \mathrm{B}_{4} \mathrm{C}$ & 3190 & 3220 & 30 & 8.69 \\
\hline Gap & $\overline{\text { Vacuum }}$ & 3170 & 3190 & 20 & 0.00 \\
\hline Vacuum Vessel & $\overline{S S 316}$ & 2870 & 3170 & 300 & 7.90 \\
\hline Gap & Vacuum & 2800 & 2870 & $\overline{70}$ & 0.00 \\
\hline \multirow[t]{5}{*}{ Toroidal Coils } & $\overline{S S 316}$ & 2760 & 2800 & 40 & 7.90 \\
\hline & Mylar & 2755 & 2760 & 5 & 1.24 \\
\hline & Magnet & 2300 & 2755 & 455 & 8.50 \\
\hline & Mylar & 2295 & 2300 & 5 & 1.24 \\
\hline & $S S 316$ & 2130 & 2295 & 165 & 7.90 \\
\hline Gap & $\overline{V a c u u m}$ & 2050 & 2130 & 80 & 0.00 \\
\hline \multirow[t]{5}{*}{ Poloidal Coil } & $\overline{S S 316}$ & 2010 & 2050 & 40 & 7.90 \\
\hline & Mylar & 2005 & 2010 & 5 & 1.24 \\
\hline & Magnet & 1555 & 2005 & 450 & 8.50 \\
\hline & Mylar & 1550 & 1555 & 5 & 1.24 \\
\hline & $S S 316$ & 1420 & 1550 & 130 & 7.90 \\
\hline
\end{tabular}

Table A.11: Radial Build of International Thermonuclear Experimental Reactor Inboard Components, Continued, Adapted from [11,21] 


\begin{tabular}{|l|r|r|r|r|r|}
\hline \hline Component & Material & $\mathbf{R}_{\mathbf{m c}_{\mathbf{i}}}$ & $\mathbf{R}_{\mathbf{m c}_{\mathbf{o}}}$ & $\mathbf{R}_{\mathbf{m c}_{\mathbf{o}}}-\mathbf{R}_{\mathbf{m c}_{\mathbf{i}}}$ & $\rho_{\mathbf{m}}$ \\
\cline { 3 - 6 } & & {$[\mathrm{mm}]$} & {$[\mathrm{mm}]$} & {$[\mathrm{mm}]$} & {$\left[\frac{t}{\mathrm{~m}^{3}}\right]$} \\
\hline First Wall & $S S 316$ & 8300 & 8305 & 5 & 7.90 \\
\hline Breeder Blanket & $\mathrm{H}_{2} \mathrm{O}$ & 8305 & 8308 & 3 & 1.00 \\
& $S S 316$ & 8308 & 8310 & 2 & 7.90 \\
& $B e$ & 8310 & 8355 & 45 & 1.85 \\
& $S S 316$ & 8355 & 8356 & 1 & 7.90 \\
& $L i_{2} \mathrm{O}$ & 8356 & 8364 & 8 & 2.01 \\
& $S S 316$ & 8364 & 8365 & 1 & 7.90 \\
& $B e$ & 8365 & 8420 & 55 & 1.85 \\
& $S S 316$ & 8420 & 8422 & 2 & 7.90 \\
& $\mathrm{H}_{\mathbf{2}} \mathrm{O}$ & 8422 & 8424 & 2 & 1.00 \\
& $S S 316$ & 8424 & 8426 & 2 & 7.90 \\
& $B e$ & 8426 & 8516 & 90 & 1.85 \\
& $S S 316$ & 8516 & 8517 & 1 & 7.90 \\
& $L i_{2} \mathrm{O}$ & 8517 & 8527 & 10 & 2.01 \\
& $S S 316$ & 8527 & 8528 & 1 & 7.90 \\
& $B e$ & 8528 & 8678 & 150 & 1.85 \\
& $S S 316$ & 8678 & 8680 & 2 & 7.90 \\
& $\mathrm{H}_{2} \mathrm{O}$ & 8680 & 8682 & 2 & 1.00 \\
\hline Back Wall & $S S 316$ & 8682 & 8732 & 50 & 7.90 \\
\hline Gap & $V a c u u m$ & 8732 & 8752 & 20 & 0.00 \\
\hline \hline
\end{tabular}

Table A.12: Radial Build of International Thermonuclear Experimental Reactor Outboard Components, Adapted from [11,21] 


\begin{tabular}{|l|r|r|r|r|r|}
\hline \hline Component & Material & $\mathbf{R}_{\mathbf{m c}_{\mathbf{i}}}$ & $\mathbf{R}_{\mathbf{m c}_{\mathbf{o}}}$ & $\mathbf{R}_{\mathbf{m c}_{\mathbf{o}}}-\mathbf{R}_{\mathbf{m c}}$ & $\rho_{\mathbf{m}}$ \\
\cline { 3 - 6 } & & {$[\mathbf{m m}]$} & {$[\mathrm{mm}]$} & {$[\mathrm{mm}]$} & {$\left[\frac{t}{\mathbf{m}^{3}}\right]$} \\
\hline Steel Shield & $S S 316$ & 8752 & 8792 & 40 & 7.90 \\
& $\mathrm{H}_{2} \mathrm{O}$ & 8792 & 8802 & 10 & 1.00 \\
& $S S 316$ & 8802 & 8912 & 110 & 7.90 \\
& $\mathrm{H}_{2} \mathrm{O}$ & 8912 & 8942 & 30 & 1.00 \\
& $S S 316$ & 8942 & 9092 & 150 & 7.90 \\
& $\mathrm{H}_{2} \mathrm{O}$ & 9092 & 9120 & 28 & 1.00 \\
& $S S 316$ & 9120 & 9270 & 150 & 7.90 \\
& $\mathrm{H}_{2} \mathrm{O}$ & 9270 & 9340 & 70 & 1.00 \\
& $S S 316$ & 9340 & 9360 & 20 & 7.90 \\
& $H_{2} \mathrm{O}$ & 9360 & 9440 & 80 & 1.00 \\
& $S S 316$ & 9440 & 9460 & 20 & 7.90 \\
\hline Lead Shield & $P b / B_{4} \mathrm{C}$ & 9460 & 9490 & 30 & 8.69 \\
\hline Gap & Vacuum & 9490 & 9510 & 20 & 0.00 \\
\hline Vacuum Vessel & $S S 316$ & 9510 & 9810 & 300 & 7.90 \\
\hline Gap & Vacuum & 9810 & 9900 & 90 & 0.00 \\
\hline Toroidal Coils & $S S 316$ & 9900 & 10040 & 140 & 7.90 \\
& Mylar & 10040 & 10045 & 5 & 1.24 \\
& Magnet & 10045 & 10845 & 800 & 8.50 \\
& Mylar & 10845 & 10850 & 5 & 1.24 \\
& $S S 316$ & 10850 & 11100 & 250 & 7.90 \\
\hline Cryostat Coolant & $H_{e}$ & 11100 & 13000 & 1900 & 0.25 \\
\hline Cryostat Vessel & SS316 & 13000 & 13020 & 20 & 7.90 \\
\hline \hline
\end{tabular}

Table A.13: Radial Build of International Thermonuclear Experimental Reactor Outboard Components, Continued, Adapted from [11,21] 


\begin{tabular}{|l|r|r|r|r|}
\hline \hline Component & \multirow{2}{*}{ Material } & Inboard & Outboard & Total \\
\cline { 2 - 5 } & & [volume-\%] & [volume-\%] & [volume-\%] \\
\hline First Wall & $R A F$ & 12.0 & 12.0 & 12.0 \\
\cline { 2 - 5 } & $\mathrm{He}$ & 88.0 & 88.0 & 88.0 \\
\hline Breeder Blanket & $R A F$ & 12.2 & 12.5 & 12.4 \\
\cline { 2 - 5 } & $\mathrm{Li}_{2} \mathrm{O}$ & 77.4 & 77.1 & 77.2 \\
\cline { 2 - 5 } & $\mathrm{He}$ & 10.4 & 10.4 & 10.4 \\
\hline Manifold & $R A F$ & 20.0 & 20.0 & 20.0 \\
\hline Steel Shield & $\mathrm{He}$ & 80.0 & 80.0 & 80.0 \\
& $\mathrm{FeCrV}$ & 80.0 & 80.0 & 80.0 \\
\hline Reactor & $\mathrm{H}_{2} \mathrm{O}$ & 20.0 & 20.0 & 20.0 \\
& $\mathrm{RAF}$ & 10.9 & 10.5 & 10.6 \\
\cline { 2 - 5 } & $\mathrm{FeCrV}$ & 18.2 & 22.2 & 20.9 \\
\cline { 2 - 5 } & $\mathrm{Li} i_{2} \mathrm{O}$ & 40.9 & 37.0 & 38.3 \\
\cline { 2 - 5 } & $\mathrm{H}_{2} \mathrm{O}$ & 4.5 & 5.5 & 5.2 \\
\cline { 2 - 5 } & $\mathrm{He}$ & 25.5 & 24.8 & 25.0 \\
\hline \hline
\end{tabular}

Table A.14: Material Composition of Reduced Activation Ferrite Helium Tokamak Components, Adapted from [19]

\begin{tabular}{|c|c|c|c|c|c|}
\hline \multirow[t]{2}{*}{ Component } & \multirow[t]{2}{*}{ Material } & $\overline{\mathbf{R}_{\mathbf{m} \mathbf{c}_{\mathrm{i}}}}$ & $\mathbf{R}_{\mathbf{m c o}_{\mathbf{o}}}$ & $\mathbf{R}_{m c_{o}}-\mathbf{R}_{m c_{i}}$ & $\rho$ \\
\hline & & {$[\mathrm{mm}]$} & {$[\mathrm{mm}]$} & {$[\mathrm{mm}]$} & {$\left[\frac{t}{m^{3}}\right]$} \\
\hline First Wall & $R A F / H e$ & 4338 & 4398 & 60 & 1.16 \\
\hline \multirow[t]{2}{*}{ Breeder Blanket } & $\mathrm{RAF} / \mathrm{He} / \mathrm{Li}_{2} \mathrm{O}$ & 3888 & 4338 & 450 & 2.28 \\
\hline & $\mathrm{RAF} / \mathrm{He} /$ & 3768 & 3888 & 120 & 3.55 \\
\hline Manifold & $R A F / H e$ & 3548 & 3768 & 220 & 1.76 \\
\hline Gap & & 3448 & 3548 & 100 & 0.00 \\
\hline Steel Shield & $\mathrm{FeCrV} / \mathrm{H}_{2} \mathrm{O}$ & 3148 & 3448 & 300 & 6.37 \\
\hline
\end{tabular}

Table A.15: Radial Build of Reduced Activation Ferrite Helium Tokamak Inboard Components, Adapted from [19] 


\begin{tabular}{|c|c|c|c|c|c|}
\hline \multirow[t]{2}{*}{ Component } & \multirow[t]{2}{*}{ Material } & $\mathbf{R}_{\mathrm{mc}_{\mathrm{i}}}$ & $\mathbf{R}_{\mathbf{m c o}_{\mathrm{o}}}$ & $\overline{\mathbf{R}_{m c_{\mathbf{o}}}-\mathbf{R}_{\mathbf{m} c_{\mathbf{i}}}}$ & $\rho_{\mathrm{m}}$ \\
\hline & & {$[\mathrm{mm}]$} & {$[\mathrm{mm}]$} & {$[\mathrm{mm}]$} & {$\left[\frac{t}{m^{3}}\right]$} \\
\hline First Wall & $R A F / H e$ & 7742 & 7802 & 60 & 1.16 \\
\hline \multirow[t]{2}{*}{ Breeder Blanket } & $\mathrm{RAF} / \mathrm{He} / \mathrm{Li}_{2} \mathrm{O}$ & 7802 & 8252 & $\overline{450}$ & $\overline{2.28}$ \\
\hline & $\mathrm{RAF} / \mathrm{He} / \mathrm{Li}_{2} \mathrm{O}$ & 8252 & 8372 & 120 & 3.55 \\
\hline Manifold & $R A F / H e$ & 8372 & 8592 & 220 & 1.76 \\
\hline Gap & Vacuum & 8592 & 8692 & 100 & 0.00 \\
\hline Steel Shield & $\mathrm{FeCrV} / \mathrm{H}_{2} \mathrm{O}$ & 8692 & 8992 & 300 & 6.37 \\
\hline
\end{tabular}

Table A.16: Radial Build of Reduced Activation Ferrite Helium Tokamak Outboard Components, Adapted from [19] 


\begin{tabular}{|c|c|c|c|c|c|}
\hline \multirow{3}{*}{ Element } & \multicolumn{5}{|c|}{ Material } \\
\hline & $\overline{\text { SS316 }}$ & SS304L & $\overline{\mathrm{RAF}}$ & $\overline{\mathrm{FeCrV}}$ & HT-9 \\
\hline & [weight-\%] & [weight-\%] & [weight-\%] & [weight-\%] & [weight-\%] \\
\hline$B$ & 0.0025 & - & 0.0010 & - & - \\
\hline$C$ & 0.0300 & 0.0300 & 0.1500 & 0.1100 & 0.2100 \\
\hline$N$ & 0.0700 & & 0.0010 & 0.0150 & 0.0037 \\
\hline$O$ & $\longrightarrow$ & - & 0.0070 & - & 0.0058 \\
\hline$A l$ & - & - & 0.0080 & 0.0430 & \\
\hline$S i$ & 0.5000 & 1.0000 & 0.2000 & 0.3000 & 0.2200 \\
\hline$P$ & 0.0350 & 0.0450 & 0.0130 & 0.0070 & \\
\hline$S$ & 0.0250 & 0.0300 & 0.0040 & 0.0150 & $\longrightarrow$ \\
\hline$K$ & & & 0.0003 & & - \\
\hline$T i$ & & & 0.1000 & 0.0030 & 0.0020 \\
\hline$V$ & & & 0.3000 & 1.5000 & 0.3400 \\
\hline$C r$ & 17.5000 & 19.0000 & 11.0000 & 2.4000 & 12.0200 \\
\hline$M n$ & 1.8000 & 2.0000 & 0.5300 & 0.3000 & 0.5000 \\
\hline $\mathrm{Fe}$ & 63.8900 & 67.8950 & 85.1600 & 95.1300 & 84.5885 \\
\hline Co & 0.2500 & - & 0.0050 & & \\
\hline$N i$ & 12.2500 & 10.0000 & 0.0060 & 0.0500 & 0.5700 \\
\hline$C u$ & 1.0000 & & 0.0030 & 0.0400 & \\
\hline$Z r$ & & & 0.0010 & & \\
\hline $\mathrm{Nb}$ & - & & 0.0001 & - & $\ldots$ \\
\hline Mo & 2.5000 & & 0.0003 & 0.0200 & 1.0300 \\
\hline$A g$ & & & 0.0001 & & \\
\hline$C d$ & & & 0.0001 & & \\
\hline$S n$ & & & 0.0030 & & \\
\hline$S b$ & & & 0.0005 & & \\
\hline$B a$ & & & 0.0020 & & \\
\hline$T b$ & - & & 0.0020 & & \\
\hline$T a$ & 0.1500 & & 0.0004 & & \\
\hline$W$ & & & 2.5000 & & 0.5100 \\
\hline$I r$ & & & 0.0020 & & \\
\hline $\mathrm{Pb}$ & & & 0.0005 & & \\
\hline$B i$ & - & - & 0.0020 & - & - \\
\hline
\end{tabular}

Table A.17: Element Composition of Structure Material, Adapted from [12, 17,19] 


\begin{tabular}{|l|r|r|}
\hline \hline \multirow{2}{*}{ Element } & \multicolumn{2}{|c|}{ Material } \\
\cline { 2 - 3 } & Copper & Lithium Oxide \\
\cline { 2 - 3 } & [weight-\%] & [weight-\%] \\
\hline $\mathrm{Li}$ & - & 43.2800 \\
$\mathrm{Na}$ & - & 56.6300 \\
$\mathrm{Al}$ & - & 0.0050 \\
$\mathrm{Si}$ & - & 0.0020 \\
$\mathrm{~S}$ & & 0.0010 \\
$\mathrm{Cl}$ & 0.0012 & \\
$K$ & - & 0.0100 \\
$\mathrm{Ca}$ & - & 0.0370 \\
$\mathrm{Mn}$ & & 0.0210 \\
$\mathrm{Fe}$ & 0.0001 & 0.0020 \\
$\mathrm{Ni}$ & 0.0022 & 0.0100 \\
$\mathrm{Cu}$ & 0.0005 & 0.0020 \\
$\mathrm{As}$ & 99.8500 & 0.0006 \\
$\mathrm{Se}$ & 0.0005 & \\
$\mathrm{Zr}$ & 0.0002 & \\
$\mathrm{Ag}$ & 0.1500 & \\
$\mathrm{Sn}$ & 0.0012 & \\
$\mathrm{Sb}$ & 0.0001 & \\
$\mathrm{Te}$ & 0.0005 & \\
$\mathrm{~Pb}$ & 0.0001 & \\
$\mathrm{Bi}$ & 0.0005 & \\
\hline \hline & 0.0001 & \\
\hline
\end{tabular}

Table A.18: Element Composition of Conductor and Breeder Material, Adapted from [19] 


\section{Appendix B}

\section{Radioactive Waste Parameters}

Tables B.1, B.2 and B.3 show specific radioactive waste parameters of the reactor components at $10 y, 100 y$ and $1,000 y$ after discharge.

Tables B.4, B.5 and B.6 show cycle, life time and decommission absolute radioactive waste parameters of the reactor components at $10 y$ after discharge. Note that decommission absolute radioactive waste parameters are given only for this time after discharge, since decommission is assumed to take place then.

Tables B.7 and B.8 show cycle and life time absolute radioactive waste parameters of the reactor components at $100 y$ after discharge.

Tables B.9 and B.10 show cycle and life time absolute radioactive waste parameters of the reactor components at $1,000 y$ after discharge.

Figures B.1, B.2, B.3 and B.4 show the specific and life time absolute radioactivity and decay power for the entire reactor, i.e. the sum of fuel, clad, duct, reflector and shield for the fast fission reactors and the sum of breeder blanket, first wall, back wall or manifold and shield for the fusion reactors. 


\begin{tabular}{|c|c|c|c|c|c|}
\hline \multirow{2}{*}{\multicolumn{2}{|c|}{ Parameter }} & \multicolumn{2}{|c|}{ Fission Reactors } & \multicolumn{2}{|c|}{ Fusion Reactors } \\
\hline & & EBR-II & IFR & ITER & RAFHT \\
\hline & & \multicolumn{2}{|c|}{ Clad } & \multicolumn{2}{|c|}{ First Wall } \\
\hline Radioactivity & {$\left[\frac{C_{i}}{m^{3}}\right]$} & $1.8 \cdot 10^{+04}$ & $1.6 \cdot 10^{+05}$ & $3.4 \cdot 10^{+06}$ & $1.0 \cdot 10^{+07}$ \\
\hline$\gamma$-Dose Rate & $\delta v / h]$ & $7.2 \cdot 10^{+00}$ & $7.8 \cdot 10^{+00}$ & $2.1 \cdot 10^{+04}$ & $3.6 \cdot 10^{+01}$ \\
\hline Decay Power & {$\left[\frac{M W}{m^{3}}\right]$} & $3.1 \cdot 10^{-05}$ & $2.2 \cdot 10^{-04}$ & $2.7 \cdot 10^{-02}$ & $1.3 \cdot 10^{-02}$ \\
\hline$B H P^{\prime \prime \prime}$ & {$[1]$} & $4.0 \cdot 10^{+07}$ & $2.3 \cdot 10^{+08}$ & $3.7 \cdot 10^{+10}$ & $1.3 \cdot 10^{+10}$ \\
\hline$R W C$ & [1] & ${ }_{A}^{8.2} \cdot 10^{-02}$ & ${ }_{B} 5.0 \cdot 10^{-06}$ & $c^{3.8} \cdot 10^{+02}$ & $c 1.4 \cdot 10^{+\infty 0}$ \\
\hline$I D R_{C}$ & $\left.\frac{s v}{h}\right]$ & $5.5 \cdot 10^{-08}$ & $3.9 \cdot 10^{-08}$ & $1.8 \cdot 10^{-04}$ & $1.2 \cdot 10^{-07}$ \\
\hline \multirow[t]{2}{*}{$I D R_{A}$} & {$\left[\frac{S_{v}}{h}\right]$} & $3.0 \cdot 10^{-03}$ & $3.1 \cdot 10^{-03}$ & $9.1 \cdot 10^{+00}$ & $1.3 \cdot 10^{-02}$ \\
\hline & & \multicolumn{2}{|c|}{ Fuel } & \multicolumn{2}{|c|}{ Breeder Blanket } \\
\hline Radioactivity & {$\left[\frac{C i}{m^{3}}\right]$} & $1.1 \cdot 10^{+06}$ & $4.7 \cdot 10^{+06}$ & $5.4 \cdot 10^{+04}$ & $4.0 \cdot 10^{+04}$ \\
\hline$\gamma$-Dose Rate & $\left.\frac{s v / h}{m^{3}}\right]$ & $8.6 \cdot 10^{+00}$ & $6.3 \cdot 10^{+01}$ & $4.6 \cdot 10^{+02}$ & $3.4 \cdot 10^{-01}$ \\
\hline Decay Power & {$\left[\frac{M W}{m^{3}}\right]$} & $2.9 \cdot 10^{-03}$ & $1.2 \cdot 10^{-02}$ & $5.5 \cdot 10^{-04}$ & $5.2 \cdot 10^{-05}$ \\
\hline$B H P^{\prime \prime \prime}$ & {$[1]$} & $7.7 \cdot 10^{+11}$ & $2.0 \cdot 10^{+12}$ & $8.0 \cdot 10^{+08}$ & $6.6 \cdot 10^{+07}$ \\
\hline$R W C$ & {$[1]$} & ${ }_{c}^{9.2 \cdot 10^{+00}}$ & $c^{4.8} \cdot 10^{+01}$ & $c 1.2 \cdot 10^{+\infty}$ & ${ }_{A} 2.9 \cdot 10^{-01}$ \\
\hline$I D R_{C}$ & $\left.\frac{s_{v}}{h}\right]$ & $8.4 \cdot 10^{-07}$ & $5.2 \cdot 10^{-06}$ & $3.8 \cdot 10^{-08}$ & $1.5 \cdot 10^{-09}$ \\
\hline \multirow[t]{2}{*}{$I D R_{A}$} & $\left.\frac{s_{v}}{h}\right]$ & $5.1 \cdot 10^{-01}$ & $2.4 \cdot 10^{+\infty 0}$ & $1.9 \cdot 10^{-01}$ & $1.3 \cdot 10^{-04}$ \\
\hline & & \multicolumn{2}{|c|}{ Duct } & \multicolumn{2}{|c|}{ Back Wall/Manifold } \\
\hline Radioactivity & $\left.\frac{i}{3}\right]$ & $1.8 \cdot 10^{+04}$ & $1.6 \cdot 10^{+05}$ & $2.1 \cdot 10^{+05}$ & $2.1 \cdot 10^{+03}$ \\
\hline$\gamma$-Dose Rate & $\left.\frac{5 v / h}{m^{3}}\right]$ & $7.0 \cdot 10^{+00}$ & $7.7 \cdot 10^{+00}$ & $2.2 \cdot 10^{+03}$ & $8.8 \cdot 10^{-01}$ \\
\hline Decay Power & $\left.\frac{M W}{m^{3}}\right]$ & $3.1 \cdot 10^{-05}$ & $2.1 \cdot 10^{-04}$ & $2.6 \cdot 10^{-03}$ & $3.7 \cdot 10^{-06}$ \\
\hline$B H P^{\prime \prime \prime}$ & [1] & $4.0 \cdot 10^{+07}$ & $2.3 \cdot 10^{+08}$ & $3.6 \cdot 10^{+09}$ & $4.1 \cdot 10^{+06}$ \\
\hline$R W C$ & [1] & ${ }_{A}^{8.0} \cdot 10^{-02}$ & ${ }_{B}^{4.9} \cdot 10^{-08}$ & $c^{1.3 \cdot 3 \cdot 10^{+\infty}}$ & ${ }_{A} 9.1 \cdot 10^{-02}$ \\
\hline$I D R_{C}$ & $\left.\frac{S_{v}}{h}\right]$ & $5.4 \cdot 10^{-08}$ & $3.9 \cdot 10^{-08}$ & $1.9 \cdot 10^{-05}$ & $7.3 \cdot 10^{-09}$ \\
\hline$I D R_{A}$ & $\left.\frac{S_{v}}{h}\right]$ & $2.9 \cdot 10^{-03}$ & $3.1 \cdot 10^{-03}$ & $9.5 \cdot 10^{-01}$ & $3.7 \cdot 10^{-04}$ \\
\hline \multirow{8}{*}{$\begin{array}{l}\text { Radioactivity } \\
\gamma \text {-Dose Rate } \\
\text { Decay Power } \\
B H P^{\prime \prime \prime} \\
R W C \\
I D R_{C} \\
I D R_{A} \\
\end{array}$} & \multirow{4}{*}{$\frac{\left[\frac{C_{i}}{m^{3}}\right]}{\left[\frac{S_{v} / h}{m^{3}}\right]}$} & \multicolumn{2}{|c|}{ Steel Shield } & \multicolumn{2}{|c|}{ Steel Shield } \\
\hline & & $5.1 \cdot 10^{+04}$ & $7.4 \cdot 10^{+04}$ & $2.1 \cdot 10^{+04}$ & $3.0 \cdot 10^{+03}$ \\
\hline & & $2.4 \cdot 10^{+01}$ & $5.5 \cdot 10^{+\infty}$ & $2.2 \cdot 10^{+02}$ & $2.1 \cdot 10^{-02}$ \\
\hline & & $9.2 \cdot 10^{-05}$ & $1.0 \cdot 10^{-04}$ & $2.6 \cdot 10^{-04}$ & $3.9 \cdot 10^{-06}$ \\
\hline & {$[1]$} & $1.3 \cdot 10^{+08}$ & $1.4 \cdot 10^{+08}$ & $3.5 \cdot 10^{+08}$ & $4.0 \cdot 10^{+08}$ \\
\hline & [1] & ${ }_{A} 3.4 \cdot 10^{-01}$ & ${ }_{C}^{1.3} \cdot 10^{+00}$ & $B^{2.5} \cdot 10^{-05}$ & ${ }_{A} 1.2 \cdot 10^{-01}$ \\
\hline & {$\left[\frac{S v}{b}\right]$} & $2.0 \cdot 10^{-07}$ & $3.8 \cdot 10^{-08}$ & $1.8 \cdot 10^{-06}$ & $1.6 \cdot 10^{-10}$ \\
\hline & {$\left[\frac{S_{v}}{h}\right]$} & $1.0 \cdot 10^{-02}$ & $2.4 \cdot 10^{-03}$ & $9.3 \cdot 10^{-02}$ & $9.1 \cdot 10^{-06}$ \\
\hline
\end{tabular}

Table B.1: Specific Radioactive Waste Parameters at 10years after Discharge 


\begin{tabular}{|c|c|c|c|c|c|}
\hline \multirow{2}{*}{\multicolumn{2}{|c|}{ Parameter }} & \multicolumn{2}{|c|}{ Fission Reactors } & \multicolumn{2}{|c|}{ Fusion Reactors } \\
\hline & & EBR-II & IFR & ITER & RAFHT \\
\hline & & \multicolumn{2}{|c|}{ Clad } & \multicolumn{2}{|c|}{ First Wall } \\
\hline Radioactivity & {$\left[\frac{C_{i}}{m^{3}}\right]$} & $1.3 \cdot 10^{+02}$ & $1.6 \cdot 10^{+02}$ & $5.7 \cdot 10^{+04}$ & $4.6 \cdot 10^{+02}$ \\
\hline$\gamma$-Dose Rate & $S_{v} / h$ & $4.5 \cdot 10^{-05}$ & $3.0 \cdot 10^{-02}$ & $1.2 \cdot 10^{+00}$ & $3.7 \cdot 10^{-01}$ \\
\hline Decay Power & {$\left[\frac{M W}{m^{3}}\right]$} & $1.9 \cdot 10^{-08}$ & $1.3 \cdot 10^{-07}$ & $1.4 \cdot 10^{-05}$ & $1.3 \cdot 10^{-08}$ \\
\hline$B H P^{\prime \prime \prime}$ & & $4.0 \cdot 10^{+06}$ & $1.7 \cdot 10^{+07}$ & $2.5 \cdot 10^{+09}$ & $9.6 \cdot 10^{+07}$ \\
\hline$R W C$ & [1] & ${ }_{A} 2.5 \cdot 10^{-02}$ & $B^{4.6} \cdot 10^{-06}$ & $c^{3.8} \cdot 10^{+02}$ & $C^{1.3} \cdot 10^{+00}$ \\
\hline$I D R_{C}$ & $\left.\frac{s_{v}}{h}\right]$ & $4.0 \cdot 10^{-13}$ & $8.0 \cdot 10^{-13}$ & $1.6 \cdot 10^{-09}$ & $7.3 \cdot 10^{-11}$ \\
\hline \multirow[t]{2}{*}{$I D R_{A}$} & $\left.\frac{S_{v}}{h}\right]$ & $3.8 \cdot 10^{-08}$ & $1.1 \cdot 10^{-04}$ & $5.6 \cdot 10^{-02}$ & $8.1 \cdot 10^{-05}$ \\
\hline & & \multicolumn{2}{|c|}{ Fuel } & \multicolumn{2}{|c|}{ Breeder Blanket } \\
\hline Radioactivity & & $1.2 \cdot 10^{+05}$ & $5.2 \cdot 10^{+05}$ & $1.7 \cdot 10^{+03}$ & $3.8 \cdot 10^{+01}$ \\
\hline$\gamma$-Dose Rate & & $6.4 \cdot 10^{-03}$ & $1.1 \cdot 10^{-02}$ & $2.1 \cdot 10^{-02}$ & $1.3 \cdot 10^{-03}$ \\
\hline Decay Power & {$\left[\frac{M W}{m^{3}}\right]$} & $3.2 \cdot 10^{-04}$ & $1.3 \cdot 10^{-03}$ & $3.4 \cdot 10^{-07}$ & $5.4 \cdot 10^{-08}$ \\
\hline$B H P^{\prime \prime \prime}$ & {$[1]$} & $8.3 \cdot 10^{+10}$ & $2.2 \cdot 10^{+11}$ & $6.4 \cdot 10^{+07}$ & $1.1 \cdot 10^{+07}$ \\
\hline$R W C$ & [1] & $c^{6.9} \cdot 10^{+\infty 0}$ & $c^{3.5} \cdot 10^{+01}$ & ${ }_{C}^{1.2} \cdot 10^{+00}$ & ${ }_{A} 2.1 \cdot 10^{-01}$ \\
\hline$I D R_{C}$ & $\left.\frac{S_{v}}{h}\right]$ & $1.0 \cdot 10^{-07}$ & $5.9 \cdot 10^{-07}$ & $3.0 \cdot 10^{-11}$ & $4.8 \cdot 10^{-13}$ \\
\hline \multirow[t]{2}{*}{$I D R_{A}$} & {$\left[\frac{S_{v}}{h}\right]$} & $6.1 \cdot 10^{-02}$ & $2.8 \cdot 10^{-01}$ & $1.7 \cdot 10^{-04}$ & $9.0 \cdot 10^{-08}$ \\
\hline & & \multicolumn{2}{|c|}{ Duct } & \multicolumn{2}{|c|}{ Back Wall/Manifold } \\
\hline Radioactivity & & $1.2 \cdot 10^{+02}$ & $1.6 \cdot 10^{+02}$ & $3.0 \cdot 10^{+03}$ & $1.1 \cdot 10^{+00}$ \\
\hline$\gamma$-Dose Rate & $\left.\frac{S v / h}{m^{3}}\right]$ & $4.5 \cdot 10^{-05}$ & $2.9 \cdot 10^{-02}$ & $1.2 \cdot 10^{-01}$ & $1.2 \cdot 10^{-04}$ \\
\hline Decay Power & {$\left[\frac{m W}{m^{3}}\right]$} & $1.9 \cdot 10^{-08}$ & $1.3 \cdot 10^{-07}$ & $8.4 \cdot 10^{-07}$ & $6.3 \cdot 10^{-10}$ \\
\hline$B H P^{\prime \prime \prime}$ & {$[1]$} & $3.9 \cdot 10^{+06}$ & $1.7 \cdot 10^{+07}$ & $1.4 \cdot 10^{+08}$ & $6.4 \cdot 10^{+04}$ \\
\hline$R W C$ & [1] & ${ }_{A} 2.5 \cdot 10^{-02}$ & ${ }_{B} 4.4 \cdot 10^{-06}$ & $c 1.3 \cdot 10^{+00}$ & ${ }_{A} 5.6 \cdot 10^{-02}$ \\
\hline$I D R_{C}$ & {$\left[\frac{s v}{b}\right]$} & $4.0 \cdot 10^{-13}$ & $8.0 \cdot 10^{-13}$ & $1.4 \cdot 10^{-10}$ & $1.0 \cdot 10^{-13}$ \\
\hline \multirow[t]{2}{*}{$I D R_{A}$} & {$\left[\frac{s_{v}}{h}\right]$} & $3.7 \cdot 10^{-08}$ & $1.0 \cdot 10^{-04}$ & $1.4 \cdot 10^{-04}$ & $1.4 \cdot 10^{-08}$ \\
\hline & & \multicolumn{2}{|c|}{ Steel Shield } & \multicolumn{2}{|c|}{ Steel Shield } \\
\hline Radioactivity & {$\left[\frac{C_{i}}{m^{3}}\right]$} & $5.6 \cdot 10^{+02}$ & $3.7 \cdot 10^{+02}$ & $4.0 \cdot 10^{+02}$ & $4.1 \cdot 10^{+\infty}$ \\
\hline$\gamma$-Dose Rate & $\left.\frac{S v / h}{m^{3}}\right]$ & $1.7 \cdot 10^{-04}$ & $5.9 \cdot 10^{-02}$ & $1.0 \cdot 10^{-02}$ & $1.1 \cdot 10^{-04}$ \\
\hline Decay Power & {$\left[\frac{M W}{m^{3}}\right]$} & $8.6 \cdot 10^{-08}$ & $2.7 \cdot 10^{-07}$ & $9.4 \cdot 10^{-08}$ & $1.1 \cdot 10^{-09}$ \\
\hline$B H P^{\prime \prime \prime}$ & {$[1]$} & $1.8 \cdot 10^{+07}$ & $3.4 \cdot 10^{+07}$ & $1.7 \cdot 10^{+07}$ & $1.6 \cdot 10^{+05}$ \\
\hline$R W C$ & {$[1]$} & ${ }_{A} 1.1 \cdot 10^{-01}$ & $C^{1.3} \cdot 10^{+\infty}$ & ${ }_{B} 9.4 \cdot 10^{-07}$ & ${ }_{A} 1.2 \cdot 10^{-01}$ \\
\hline$I D R_{C}$ & $\left.\frac{S_{v}}{h}\right]$ & $1.5 \cdot 10^{-12}$ & $1.1 \cdot 10^{-12}$ & $1.4 \cdot 10^{-11}$ & $4.5 \cdot 10^{-15}$ \\
\hline$I D R_{A}$ & $\frac{s_{v}}{h}$ & $1.5 \cdot 10^{-07}$ & $2.0 \cdot 10^{-04}$ & $1.1 \cdot 10^{-05}$ & $1.3 \cdot 10^{-07}$ \\
\hline
\end{tabular}

Table B.2: Specific Radioactive Waste Parameters at 100years after Discharge 


\begin{tabular}{|c|c|c|c|c|c|}
\hline \multirow{2}{*}{\multicolumn{2}{|c|}{ Parameter }} & \multicolumn{2}{|c|}{ Fission Reactors } & \multicolumn{2}{|c|}{ Fusion Reactors } \\
\hline & & EBR-II & IFR & ITER & RAFHT \\
\hline & & \multicolumn{2}{|c|}{ Clad } & \multicolumn{2}{|c|}{ First Wall } \\
\hline Radioactivity & {$\left[\frac{C i}{m^{3}}\right]$} & $7.9 \cdot 10^{+00}$ & $8.5 \cdot 10^{+01}$ & $5.9 \cdot 10^{+03}$ & $4.1 \cdot 10^{+01}$ \\
\hline$\gamma$-Dose Rate & $\left.\frac{S v / h}{m^{3}}\right]$ & $8.3 \cdot 10^{-09}$ & $2.8 \cdot 10^{-02}$ & $8.9 \cdot 10^{-01}$ & $6.1 \cdot 10^{-04}$ \\
\hline Decay Power & & $3.6 \cdot 10^{-10}$ & $1.1 \cdot 10^{-05}$ & $4.9 \cdot 10^{-06}$ & $2.8 \cdot 10^{-08}$ \\
\hline$B H P^{\prime \prime \prime}$ & [1] & $4.3 \cdot 10^{+04}$ & $1.4 \cdot 10^{+07}$ & $5.4 \cdot 10^{+08}$ & $9.3 \cdot 10^{+06}$ \\
\hline$R W C$ & [1] & $A^{4.0} \cdot 10^{-03}$ & ${ }_{B} 4.4 \cdot 10^{-08}$ & $c^{3.8} \cdot 10^{+02}$ & $C^{1.1} \cdot 10^{+\infty 0}$ \\
\hline$I D R_{C}$ & $\left.\frac{S v}{h}\right]$ & $2.7 \cdot 10^{-10}$ & $1.4 \cdot 10^{-07}$ & $7.2 \cdot 10^{-05}$ & $6.9 \cdot 10^{-07}$ \\
\hline \multirow[t]{2}{*}{$I D R_{A}$} & $\frac{\xi_{v}^{2}}{h}$ & $9.2 \cdot 10^{-10}$ & $1.0 \cdot 10^{-04}$ & $5.5 \cdot 10^{-02}$ & $7.7 \cdot 10^{-05}$ \\
\hline & & \multicolumn{2}{|c|}{ Fuel } & \multicolumn{2}{|c|}{ Breeder Blanket } \\
\hline Radioactivity & & $8.6 \cdot 10^{+01}$ & $4.5 \cdot 10^{+02}$ & $8.8 \cdot 10^{+01}$ & $6.5 \cdot 10^{+00}$ \\
\hline$\gamma$-Dose Rate & & $6.0 \cdot 10^{-03}$ & $4.0 \cdot 10^{-03}$ & $1.5 \cdot 10^{-02}$ & $5.1 \cdot 10^{-06}$ \\
\hline Decay Power & & $8.2 \cdot 10^{-08}$ & $5.6 \cdot 10^{-07}$ & $6.7 \cdot 10^{-08}$ & $5.4 \cdot 10^{-09}$ \\
\hline$B H P^{\prime \prime \prime}$ & [1] & $4.8 \cdot 10^{+08}$ & $2.6 \cdot 10^{+07}$ & $8.7 \cdot 10^{+06}$ & $1.1 \cdot 10^{+06}$ \\
\hline$R W C$ & {$[1]$} & ${ }_{C} 6.6 \cdot 10^{+00}$ & ${ }_{c} 3.3 \cdot 10^{+01}$ & ${ }_{C}^{1.2} \cdot 10^{+\infty}$ & ${ }_{A}^{9.6} \cdot 10^{-02}$ \\
\hline$I D R_{C}$ & $\left.\frac{S v}{h}\right]$ & $6.0 \cdot 10^{-07}$ & $2.4 \cdot 10^{-08}$ & $3.1 \cdot 10^{-07}$ & $4.3 \cdot 10^{-09}$ \\
\hline \multirow[t]{2}{*}{$I D R_{A}$} & {$\left[\frac{s_{v}}{h}\right]$} & $1.1 \cdot 10^{-03}$ & $6.0 \cdot 10^{-03}$ & $1.7 \cdot 10^{-04}$ & $6.3 \cdot 10^{-08}$ \\
\hline & & \multicolumn{2}{|c|}{ Duct } & \multicolumn{2}{|c|}{ Back Wall/Manifold } \\
\hline Radioactivity & $\left.\frac{z_{i}}{3}\right]$ & $7.8 \cdot 10^{+\infty}$ & $8.4 \cdot 10^{+01}$ & $3.1 \cdot 10^{+02}$ & $8.4 \cdot 10^{-02}$ \\
\hline$\gamma$-Dose Rate & $\left.\frac{S v / h}{m^{3}}\right]$ & $8.1 \cdot 10^{-09}$ & $2.7 \cdot 10^{-02}$ & $8.3 \cdot 10^{-02}$ & $3.5 \cdot 10^{-08}$ \\
\hline Decay Power & & $3.6 \cdot 10^{-10}$ & $1.1 \cdot 10^{-07}$ & $3.3 \cdot 10^{-07}$ & $2.0 \cdot 10^{-11}$ \\
\hline$B H P^{\prime \prime \prime}$ & {$[1]$} & $4.3 \cdot 10^{+04}$ & $1.3 \cdot 10^{+07}$ & $4.2 \cdot 10^{+07}$ & $3.3 \cdot 10^{+03}$ \\
\hline$R W C$ & [1] & $A^{4.0} \cdot 10^{-03}$ & ${ }_{B}^{4.4} \cdot 10^{-06}$ & $C^{1.2} \cdot 10^{+00}$ & ${ }_{A} 1.2 \cdot 10^{-02}$ \\
\hline$I D R_{C}$ & $\left.\frac{s v}{h}\right]$ & $2.7 \cdot 10^{-10}$ & $1.4 \cdot 10^{-07}$ & $4.9 \cdot 10^{-07}$ & $1.2 \cdot 10^{-09}$ \\
\hline$I D R_{A}$ & $\frac{s_{v}}{h}$ & $9.1 \cdot 10^{-10}$ & $1.0 \cdot 10^{-04}$ & $1.4 \cdot 10^{-04}$ & $1.9 \cdot 10^{-09}$ \\
\hline \multirow{3}{*}{ Radioactivity } & \multirow[b]{2}{*}[\frac{Ci}{m^{3}}]{} & \multicolumn{2}{|c|}{ Steel Shield } & \multicolumn{2}{|c|}{ Steel Shield } \\
\hline & & $3.6 \cdot 10^{+01}$ & $2.2 \cdot 10^{+02}$ & $3.0 \cdot 10^{+01}$ & $8.1 \cdot 10^{-01}$ \\
\hline & $\left.\frac{S v h}{m^{3}}\right]$ & $3.8 \cdot 10^{-08}$ & $5.5 \cdot 10^{-02}$ & $7.3 \cdot 10^{-03}$ & $9.4 \cdot 10^{-05}$ \\
\hline Decay Power & {$\left[\frac{M W}{m^{3}}\right]$} & $1.7 \cdot 10^{-00}$ & $2.3 \cdot 10^{-07}$ & $2.9 \cdot 10^{-08}$ & $5.1 \cdot 10^{-10}$ \\
\hline$B H P^{\prime \prime \prime}$ & [1] & $2.0 \cdot 10^{+05}$ & $2.7 \cdot 10^{+07}$ & $3.7 \cdot 10^{+06}$ & $4.8 \cdot 10^{+04}$ \\
\hline$R W C$ & [1] & ${ }_{A}^{1.8} \cdot 10^{-02}$ & $C^{1.3} \cdot 10^{+\infty 0}$ & ${ }_{B} 9.1 \cdot 10^{-07}$ & ${ }_{A} 1.2 \cdot 10^{-01}$ \\
\hline$I D R_{C}$ & {$\left[\frac{S v}{h}\right]$} & $1.2 \cdot 10^{-09}$ & $2.3 \cdot 10^{-07}$ & $3.3 \cdot 10^{-08}$ & $4.0 \cdot 10^{-10}$ \\
\hline$I D R_{A}$ & {$\left[\frac{s_{v}^{2}}{h}\right]$} & $4.2 \cdot 10^{-09}$ & $2.0 \cdot 10^{-04}$ & $1.0 \cdot 10^{-05}$ & $1.3 \cdot 10^{-07}$ \\
\hline
\end{tabular}

Table B.3: Specific Radioactive Waste Parameters at 1000years after Discharge 


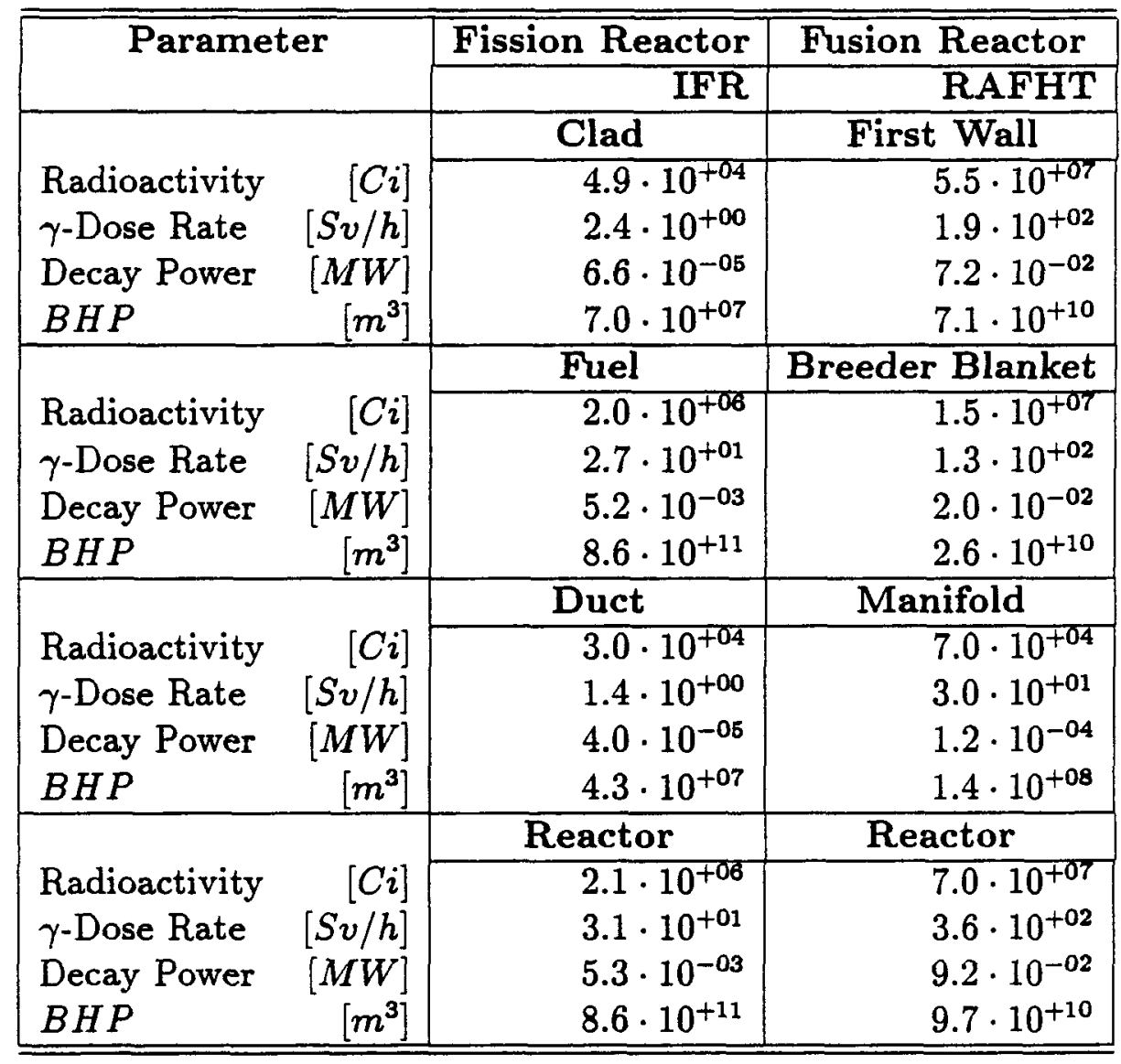

Table B.4: Absolute Radioactive Waste Parameters for Cycle Radioactive Waste at 10years after Discharge 


\begin{tabular}{|c|c|c|c|c|c|}
\hline \multirow{2}{*}{\multicolumn{2}{|c|}{ Parameter }} & \multicolumn{2}{|c|}{ Fission Reactors } & \multicolumn{2}{|c|}{ Fusion Reactors } \\
\hline & & EBR-II & IFR & ITER & RAFHT \\
\hline \multirow{5}{*}{$\begin{array}{l}\text { Radioactivity } \\
\gamma \text {-Dose Rate } \\
\text { Decay Power } \\
B H P \\
\end{array}$} & \multirow{5}{*}{$\begin{array}{r}{[\mathrm{Ci}]} \\
{[S v / h]} \\
{[M W]} \\
{\left[\mathrm{m}^{3}\right]} \\
\end{array}$} & \multicolumn{2}{|c|}{ Clad } & \multicolumn{2}{|c|}{ First Wall } \\
\hline & & $3.1 \cdot 10^{+03}$ & $1.5 \cdot 10^{+06}$ & $1.3 \cdot 10^{+07}$ & $2.7 \cdot 10^{+08}$ \\
\hline & & $1.2 \cdot 10^{+\infty}$ & $7.2 \cdot 10^{+01}$ & $8.0 \cdot 10^{+04}$ & $9.7 \cdot 10^{+02}$ \\
\hline & & $5.3 \cdot 10^{-08}$ & $2.0 \cdot 10^{-03}$ & $1.0 \cdot 10^{-01}$ & $3.6 \cdot 10^{-01}$ \\
\hline & & $6.9 \cdot 10^{+06}$ & $2.1 \cdot 10^{+09}$ & $1.4 \cdot 10^{+11}$ & $3.6 \cdot 10^{+11}$ \\
\hline \multirow{5}{*}{$\begin{array}{l}\text { Radioactivity } \\
\gamma \text {-Dose Rate } \\
\text { Decay Power } \\
B H P\end{array}$} & \multirow{5}{*}{$\begin{array}{r}{[C i]} \\
{[S v / h]} \\
{[M W]} \\
{\left[\mathrm{m}^{3}\right]} \\
\end{array}$} & \multicolumn{2}{|c|}{ Fuel } & \multicolumn{2}{|c|}{ Breeder Blanket } \\
\hline & & $7.5 \cdot 10^{+05}$ & $6.0 \cdot 10^{+07}$ & $1.2 \cdot 10^{+07}$ & $7.7 \cdot 10^{+07}$ \\
\hline & & $6.0 \cdot 10^{+\infty 0}$ & $8.1 \cdot 10^{+02}$ & $1.0 \cdot 10^{+05}$ & $6.7 \cdot 10^{+02}$ \\
\hline & & $2.0 \cdot 10^{-03}$ & $1.6 \cdot 10^{-01}$ & $1.2 \cdot 10^{-01}$ & $1.0 \cdot 10^{-01}$ \\
\hline & & $5.3 \cdot 10^{+11}$ & $2.6 \cdot 10^{+13}$ & $1.8 \cdot 10^{+11}$ & $1.3 \cdot 10^{+11}$ \\
\hline \multirow{5}{*}{$\begin{array}{l}\text { Radioactivity } \\
\gamma \text {-Dose Rate } \\
\text { Decay Power } \\
B H P\end{array}$} & \multirow{5}{*}{$\begin{array}{r}{[C i]} \\
{[S v / h]} \\
{[M W]} \\
{\left[\mathrm{m}^{3}\right]} \\
\end{array}$} & \multicolumn{2}{|c|}{ Duct } & \multicolumn{2}{|c|}{ Back Wall/Manifold } \\
\hline & & $5.0 \cdot 10^{+03}$ & $8.9 \cdot 10^{+05}$ & $9.0 \cdot 10^{+06}$ & $3.5 \cdot 10^{+05}$ \\
\hline & & $2.0 \cdot 10^{+\infty}$ & $10^{+01}$ & $9.5 \cdot 10^{+04}$ & $1.5 \cdot 10^{+02}$ \\
\hline & & $8.6 \cdot 10^{-06}$ & $1.2 \cdot 10^{-03}$ & $1.1 \cdot 10^{-01}$ & $6.2 \cdot 10^{-04}$ \\
\hline & & $1.1 \cdot 10^{+07}$ & $1.3 \cdot 10^{+09}$ & $1.5 \cdot 10^{+11}$ & $6.8 \cdot 10^{+08}$ \\
\hline \multirow{5}{*}{$\begin{array}{l}\text { Radioactivity } \\
\gamma \text {-Dose Rate } \\
\text { Decay Power } \\
B H P\end{array}$} & \multirow{5}{*}{$\begin{array}{r}{[C i]} \\
{[S v / h]} \\
{[M W]} \\
{\left[\mathrm{m}^{3}\right]}\end{array}$} & \multicolumn{2}{|c|}{ Steel Shield } & \multicolumn{2}{|c|}{ Steel Shield } \\
\hline & & $5.7 \cdot 10^{+04}$ & $4.7 \cdot 10^{+05}$ & $6.4 \cdot 10^{+08}$ & $5.5 \cdot 10^{+05}$ \\
\hline & & $2.7 \cdot 10^{+01}$ & $3.5 \cdot 10^{+01}$ & $6.8 \cdot 10^{+04}$ & $10^{+00}$ \\
\hline & & $1.0 \cdot 10^{-04}$ & $6.6 \cdot 10^{-04}$ & $7.9 \cdot 10^{-02}$ & $7.2 \cdot 10^{-04}$ \\
\hline & & $1.5 \cdot 10^{+08}$ & $8.9 \cdot 10^{+08}$ & $1.1 \cdot 10^{+11}$ & $7.4 \cdot 10^{+08}$ \\
\hline \multirow{5}{*}{$\begin{array}{l}\text { Radioactivity } \\
\gamma \text {-Dose Rate } \\
\text { Decay Power } \\
B H P\end{array}$} & \multirow{5}{*}{$\begin{array}{r}{[C i]} \\
{[S v / h]} \\
{[M W]} \\
{\left[\mathrm{m}^{3}\right]} \\
\end{array}$} & \multicolumn{2}{|c|}{ Reactor } & \multicolumn{2}{|c|}{ Reactor } \\
\hline & & $8.2 \cdot 10^{+05}$ & $6.3 \cdot 10^{+07}$ & $4.1 \cdot 10^{+07}$ & $3.5 \cdot 10^{+08}$ \\
\hline & & $3.6 \cdot 10^{+01}$ & $9.6 \cdot 10^{+02}$ & $3.5 \cdot 10^{+05}$ & $1.8 \cdot 10^{+03}$ \\
\hline & & $2.1 \cdot 10^{-03}$ & $1.6 \cdot 10^{-01}$ & $4.2 \cdot 10^{-01}$ & $4.6 \cdot 10^{-01}$ \\
\hline & & $5.3 \cdot 10^{+11}$ & $2.6 \cdot 10^{+13}$ & $5.8 \cdot 10^{+11}$ & $4.9 \cdot 10^{+11}$ \\
\hline
\end{tabular}

Table B.5: Absolute Radioactive Waste Parameters for Life Time Radioactive Waste at 10years after Discharge 


\begin{tabular}{|c|c|c|c|c|c|}
\hline \multirow{2}{*}{\multicolumn{2}{|c|}{ Para }} & \multicolumn{2}{|c|}{ Fission Rea } & \multicolumn{2}{|c|}{ Fusion Reg } \\
\hline & & & & & तर \\
\hline \multirow{5}{*}{$\begin{array}{l}\text { Radioactivity } \\
\gamma \text {-Dose Rate } \\
\text { Decay Power } \\
B H P\end{array}$} & \multirow{5}{*}{$\begin{array}{r}{[C i]} \\
{[S v / h]} \\
{[M W]} \\
{\left[\mathrm{m}^{3}\right]}\end{array}$} & \multicolumn{2}{|c|}{ Clad } & \multicolumn{2}{|c|}{ First Wall } \\
\hline & & $\pi$ & & $.10^{-1}$ & 55 \\
\hline & & & & & \\
\hline & & & & & \\
\hline & & & & 4. & \\
\hline \multirow{5}{*}{$\begin{array}{l}\text { Radioactivity } \\
\gamma \text {-Dose Rate } \\
\text { Decay Power } \\
B H P\end{array}$} & \multirow{5}{*}{$\begin{array}{r}{[\mathrm{Ci}]} \\
{[S v / h]} \\
{[M W]} \\
{\left[\mathrm{m}^{3}\right]} \\
\end{array}$} & \multicolumn{2}{|c|}{ Fuel } & \multicolumn{2}{|c|}{ Breeder Blanket } \\
\hline & & 05 & & $1.2 \cdot 1$ & \\
\hline & & & & & \\
\hline & & & & 2 & \\
\hline & & & & 1.8. & \\
\hline \multirow{5}{*}{$\begin{array}{l}\text { Radioactivity } \\
\gamma \text {-Dose Rate } \\
\text { Decay Power } \\
B H P\end{array}$} & \multirow{5}{*}{$\begin{array}{r}{[C i]} \\
{[S v / h]} \\
{[M W]} \\
{\left[\mathrm{m}^{3}\right]}\end{array}$} & \multicolumn{2}{|c|}{ Duct } & \multicolumn{2}{|c|}{ Back Wall/Manifold } \\
\hline & & 03 & & 9.0 . & \\
\hline & & & & & \\
\hline & & . & & & \\
\hline & & $\cdot 1$ & & $5 \cdot 10$ & \\
\hline \multirow{5}{*}{$\begin{array}{l}\text { Radioactivity } \\
\gamma \text {-Dose Rate } \\
\text { Decay Power } \\
B H P\end{array}$} & \multirow{5}{*}{$\begin{array}{r}{[C i]} \\
{[S v / h]} \\
{[M W]} \\
{\left[\mathrm{m}^{3}\right]} \\
\end{array}$} & \multicolumn{2}{|c|}{ Steel Shi } & \multicolumn{2}{|c|}{ eel Shie } \\
\hline & & $.7 \cdot 10$ & $10^{+05}$ & $6.4 \cdot 10^{+06}$ & $F$ \\
\hline & & & & 8 . & \\
\hline & & $\cdot 1$ & & $7.9 \cdot 10^{-02}$ & \\
\hline & & $0^{\text {Tud }}$ & 8.9 . & $.1 \cdot 10^{+11}$ & \\
\hline \multirow{5}{*}{$\begin{array}{l}\text { Radioactivity } \\
\gamma \text {-Dose Rate } \\
\text { Decay Power } \\
B H P\end{array}$} & \multirow{5}{*}{$\begin{array}{r}{[C i]} \\
{[S v / h]} \\
{[M W]}\end{array}$} & \multicolumn{2}{|c|}{ Reactor } & \multicolumn{2}{|c|}{ Reactor } \\
\hline & & $2 \cdot 10^{+}$ & $8.9 \cdot 10^{+06}$ & $4.1 \cdot 10$ & \\
\hline & & 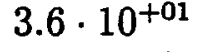 & & $3.5 \cdot 1$ & \\
\hline & & 1 . & & $4.2 \cdot$ & \\
\hline & & $3 \cdot 1$ & 3.4 & $5.8 \cdot 10$ & $9.8 \cdot 10^{+}$ \\
\hline
\end{tabular}

Table B.6: Absolute Radioactive Waste Parameters for Decommission Radioactive Waste at 10years after Discharge 


\begin{tabular}{|lr|r|r|}
\hline \hline \multicolumn{2}{|c|}{ Parameter } & Fission Reactor & Fusion Reactor \\
\cline { 3 - 4 } & & IFR & RAFHT \\
\hline & & Clad & \multicolumn{1}{c|}{ First Wall } \\
\cline { 3 - 4 } Radioactivity & {$[\mathrm{Ci}]$} & $5.0 \cdot 10^{+01}$ & $2.5 \cdot 10^{+03}$ \\
$\gamma$-Dose Rate & {$[\mathrm{Sv} / \mathrm{h}]$} & $9.0 \cdot 10^{-03}$ & $2.0 \cdot 10^{+00}$ \\
Decay Power & {$[\mathrm{MW}]$} & $4.0 \cdot 10^{-08}$ & $7.2 \cdot 10^{-06}$ \\
$B H P$ & {$\left[\mathrm{~m}^{3}\right]$} & $5.2 \cdot 10^{+06}$ & $5.3 \cdot 10^{+08}$ \\
\hline & & Fuel & Breeder Blanket \\
\cline { 3 - 4 } Radioactivity & {$[\mathrm{Ci}]$} & $2.2 \cdot 10^{+05}$ & $1.5 \cdot 10^{+04}$ \\
$\gamma$-Dose Rate & {$[S v / h]$} & $4.7 \cdot 10^{-03}$ & $5.1 \cdot 10^{-01}$ \\
Decay Power & {$[\mathrm{MW}]$} & $5.7 \cdot 10^{-04}$ & $2.1 \cdot 10^{-05}$ \\
$B H P$ & {$\left[\mathrm{~m}^{3}\right]$} & $9.3 \cdot 10^{+10}$ & $4.3 \cdot 10^{+09}$ \\
\hline & & Duct & Manifold \\
\cline { 3 - 4 } Radioactivity & {$[\mathrm{Ci}]$} & $3.0 \cdot 10^{+01}$ & $3.9 \cdot 10^{+01}$ \\
$\gamma$-Dose Rate & {$[S v / h]$} & $5.5 \cdot 10^{-03}$ & $4.0 \cdot 10^{-03}$ \\
Decay Power & {$[\mathrm{MW}]$} & $2.4 \cdot 10^{-08}$ & $2.1 \cdot 10^{-08}$ \\
$B H P$ & {$\left[\mathrm{~m}^{3}\right]$} & $3.2 \cdot 10^{+06}$ & $2.1 \cdot 10^{+06}$ \\
\hline & & Reactor & Reactor \\
\cline { 3 - 4 } Radioactivity & {$[\mathrm{Ci}]$} & $2.2 \cdot 10^{+05}$ & $1.7 \cdot 10^{+04}$ \\
$\gamma$-Dose Rate & {$[S v / h]$} & $1.9 \cdot 10^{-02}$ & $2.5 \cdot 10^{+00}$ \\
Decay Power & {$[\mathrm{MW}]$} & $5.7 \cdot 10^{-04}$ & $2.8 \cdot 10^{-05}$ \\
$B H P$ & {$\left[\mathrm{~m}^{3}\right]$} & $9.3 \cdot 10^{+10}$ & $4.9 \cdot 10^{+09}$ \\
\hline \hline
\end{tabular}

Table B.7: Absolute Radioactive Waste Parameters for Cycle Radioactive Waste at 100years after Discharge 


\begin{tabular}{|c|c|c|c|c|c|}
\hline \multirow{2}{*}{\multicolumn{2}{|c|}{ Para }} & \multicolumn{2}{|c|}{ Fission Reactors } & \multicolumn{2}{|c|}{ Fusion Reactors } \\
\hline & & EBR-II & IFR & ITER & RAFHT \\
\hline \multirow{5}{*}{$\begin{array}{l}\text { Radioactivity } \\
\gamma \text {-Dose Rate } \\
\text { Decay Power } \\
B H P\end{array}$} & \multirow[b]{2}{*}[Ci]{} & \multicolumn{2}{|c|}{ Clad } & \multicolumn{2}{|c|}{ First Wall } \\
\hline & & \multicolumn{2}{|c|}{$\begin{array}{l}01 \\
0+01\end{array}$} & $10^{+05}$ & $1.3 \cdot 10^{+04}$ \\
\hline & {$[S v / h]$} & $7.8 \cdot 10^{-08}$ & $10^{-01}$ & $4.5 \cdot 10^{+00}$ & $1.0 \cdot 10^{+01}$ \\
\hline & {$[M W]$} & $3.3 \cdot 10^{-09}$ & $1.2 \cdot 10^{-08}$ & $5.2 \cdot 10^{-05}$ & $3.6 \cdot 10^{-05}$ \\
\hline & & $8 \cdot 10^{+05}$ & $1.6 \cdot 10^{+08}$ & $9.3 \cdot 10^{+09}$ & $2.6 \cdot 10^{+09}$ \\
\hline \multirow{5}{*}{$\begin{array}{l}\text { Radioactivity } \\
\gamma \text {-Dose Rate } \\
\text { Decay Power } \\
B H P\end{array}$} & \multirow[b]{2}{*}[Ci]{} & \multicolumn{2}{|c|}{ Fuel } & \multicolumn{2}{|c|}{ Breeder Blanket } \\
\hline & & $8.1 \cdot 10^{+04}$ & $6.7 \cdot 10^{+06}$ & $3.8 \cdot 10^{+05}$ & $7.4 \cdot 10^{+04}$ \\
\hline & $S v / h]$ & $4.4 \cdot 10^{-03}$ & $1.4 \cdot 10^{-01}$ & $4.8 \cdot 10^{+00}$ & $2.5 \cdot 10^{+00}$ \\
\hline & {$[M W]$} & $2.2 \cdot 10^{-04}$ & $0^{-02}$ & $7.7 \cdot 10^{-05}$ & $10^{-04}$ \\
\hline & {$\left[m^{3}\right]$} & $.8 \cdot 10^{+10}$ & $2.8 \cdot 10^{+12}$ & $1.5 \cdot 10^{+10}$ & $2.2 \cdot 10^{+10}$ \\
\hline \multirow{5}{*}{$\begin{array}{l}\text { Radioactivity } \\
\gamma \text {-Dose Rate } \\
\text { Decay Power } \\
B H P \\
\end{array}$} & \multirow{5}{*}{$\begin{array}{r}{[C i]} \\
S v / h] \\
M W] \\
{\left[m^{3}\right]}\end{array}$} & \multicolumn{2}{|c|}{ Duct } & \multicolumn{2}{|c|}{ Back Wall/Manifold } \\
\hline & & $10^{+01}$ & $10^{+02}$ & $1.3 \cdot 10^{+05}$ & $10^{+02}$ \\
\hline & & $1.3 \cdot 10^{-05}$ & $1.6 \cdot 10^{-01}$ & $4.9 \cdot 10^{+00}$ & $2.0 \cdot 10^{-02}$ \\
\hline & & $5.3 \cdot 10^{-09}$ & $7.3 \cdot 10^{-08}$ & $3.6 \cdot 10^{-05}$ & $1.1 \cdot 10^{-07}$ \\
\hline & & $1.1 \cdot 10^{+06}$ & $9.5 \cdot 10^{+07}$ & $6.0 \cdot 10^{+09}$ & $1.1 \cdot 10^{+07}$ \\
\hline \multirow{5}{*}{$\begin{array}{l}\text { Radioactivity } \\
\gamma \text {-Dose Rate } \\
\text { Decay Power } \\
B H P\end{array}$} & \multirow{5}{*}{$\begin{array}{r}{[C i]} \\
{[S v / h]} \\
{[M W]} \\
{\left[m^{3}\right]}\end{array}$} & \multicolumn{2}{|c|}{ Steel Shield } & \multicolumn{2}{|c|}{ Steel Shield } \\
\hline & & $6.3 \cdot 10^{+02}$ & $2.4 \cdot 10^{+03}$ & $1.2 \cdot 10^{+05}$ & $7.5 \cdot 10^{+02}$ \\
\hline & & $1.8 \cdot 10^{-04}$ & $3.8 \cdot 10^{-01}$ & $3.2 \cdot 10^{+00}$ & $2.1 \cdot 10^{-02}$ \\
\hline & & $9.6 \cdot 10^{-08}$ & $1.8 \cdot 10^{-06}$ & $2.9 \cdot 10^{-05}$ & $2.0 \cdot 10^{-07}$ \\
\hline & & $2.0 \cdot 10^{+07}$ & $0^{+08}$ & $5.1 \cdot 10^{+09}$ & $3.0 \cdot 10^{+07}$ \\
\hline \multirow{5}{*}{$\begin{array}{l}\text { Radioactivity } \\
\gamma \text {-Dose Rate } \\
\text { Decay Power } \\
B H P\end{array}$} & \multirow{5}{*}{$\begin{array}{r}{[C i]} \\
{[S v / h]} \\
{[M W]} \\
{\left[m^{3}\right]}\end{array}$} & \multicolumn{2}{|c|}{ Reactor } & \multicolumn{2}{|c|}{ Reactor } \\
\hline & & $8.1 \cdot 10^{+04}$ & $6.7 \cdot 10^{+06}$ & $8.5 \cdot 10^{+05}$ & $8.8 \cdot 10^{+04}$ \\
\hline & & $4.7 \cdot 10^{-03}$ & $9.5 \cdot 10^{-01}$ & $1.8 \cdot 10^{+01}$ & $1.3 \cdot 10^{+01}$ \\
\hline & & $2.2 \cdot 10^{-04}$ & $1.7 \cdot 10^{-02}$ & $1.9 \cdot 10^{-04}$ & $1.4 \cdot 10^{-04}$ \\
\hline & & $5.8 \cdot 10^{+10}$ & $2.8 \cdot 10^{+12}$ & $3.5 \cdot 10^{+}$ & $2.4 \cdot 10^{+10}$ \\
\hline
\end{tabular}

Table B.8: Absolute Radioactive Waste Parameters for Life Time Radioactive Waste at 100years after Discharge 


\begin{tabular}{|c|c|c|c|}
\hline \multirow{2}{*}{\multicolumn{2}{|c|}{ Parameter }} & Fission Reactor & Fusion Reactor \\
\hline & & IFR & RAFHT \\
\hline \multirow{5}{*}{$\begin{array}{l}\text { Radioactivity } \\
\gamma \text {-Dose Rate } \\
\text { Decay Power } \\
B H P\end{array}$} & \multirow{5}{*}{$\begin{array}{r}{[C i]} \\
{[S v / h]} \\
{[M W]} \\
{\left[\mathrm{m}^{3}\right]}\end{array}$} & Clad & First Wall \\
\hline & & $2.6 \cdot 10^{+01}$ & $2.2 \cdot 10^{+02}$ \\
\hline & & $8.4 \cdot 10^{-03}$ & $3.4 \cdot 10^{-03}$ \\
\hline & & $3.4 \cdot 10^{-08}$ & $1.5 \cdot 10^{-07}$ \\
\hline & & $4.2 \cdot 10^{+06}$ & $5.1 \cdot 10^{+07}$ \\
\hline \multirow{5}{*}{$\begin{array}{l}\text { Radioactivity } \\
\gamma \text {-Dose Rate } \\
\text { Decay Power } \\
B H P\end{array}$} & \multirow{5}{*}{$\begin{array}{r}{[\mathrm{Ci}]} \\
{[S v / h]} \\
{[M W]} \\
{\left[\mathrm{m}^{3}\right]} \\
\end{array}$} & Fuel & Breeder Blanket \\
\hline & & $1.9 \cdot 10^{+02}$ & $2.5 \cdot 10^{+03}$ \\
\hline & & $1.7 \cdot 10^{-03}$ & $2.0 \cdot 10^{-03}$ \\
\hline & & $2.4 \cdot 10^{-07}$ & $2.1 \cdot 10^{-06}$ \\
\hline & & $1.1 \cdot 10^{+07}$ & $4.2 \cdot 10^{+08}$ \\
\hline \multirow{5}{*}{$\begin{array}{l}\text { Radioactivity } \\
\gamma \text {-Dose Rate } \\
\text { Decay Power } \\
B H P\end{array}$} & \multirow{5}{*}{$\begin{array}{r}{[C i]} \\
{[S v / h]} \\
{[M W]} \\
{\left[m^{3}\right]}\end{array}$} & Duct & Manifold \\
\hline & & $1.6 \cdot 10^{+01}$ & $2.8 \cdot 10^{+00}$ \\
\hline & & $5.1 \cdot 10^{-03}$ & $1.2 \cdot 10^{-04}$ \\
\hline & & $2.1 \cdot 10^{-08}$ & $6.6 \cdot 10^{-10}$ \\
\hline & & $2.5 \cdot 10^{+06}$ & $1.1 \cdot 10^{+05}$ \\
\hline \multirow{5}{*}{$\begin{array}{l}\text { Radioactivity } \\
\gamma \text {-Dose Rate } \\
\text { Decay Power } \\
B H P\end{array}$} & \multirow{5}{*}{$\begin{array}{r}{[C i]} \\
{[S v / h]} \\
{[M W]} \\
{\left[m^{3}\right]}\end{array}$} & Reactor & Reactor \\
\hline & & $2.4 \cdot 10^{+02}$ & $2.8 \cdot 10^{+03}$ \\
\hline & & $1.5 \cdot 10^{-02}$ & $5.5 \cdot 10^{-03}$ \\
\hline & & $3.0 \cdot 10^{-07}$ & $2.3 \cdot 10^{-06}$ \\
\hline & & $1.8 \cdot 10^{+07}$ & $4.7 \cdot 10^{+08}$ \\
\hline
\end{tabular}

Table B.9: Absolute Radioactive Waste Parameters for Cycle Radioactive Waste at 1000 years after Discharge 


\begin{tabular}{|c|c|c|c|c|c|}
\hline \multirow{2}{*}{\multicolumn{2}{|c|}{$\operatorname{ar}$}} & \multicolumn{2}{|c|}{ Fission Reactors } & \multicolumn{2}{|c|}{ Fusion Reactors } \\
\hline & & EBR-II & IFR & ITER & $\mathbf{R}$ \\
\hline \multirow{5}{*}{$\begin{array}{l}\text { Radioactivity } \\
\gamma \text {-Dose Rate } \\
\text { Decay Power } \\
B H P\end{array}$} & \multirow{5}{*}{$\begin{array}{r}{[C i]} \\
{[S v / h]} \\
{[M W]} \\
{\left[\mathrm{m}^{3}\right]} \\
\end{array}$} & \multicolumn{2}{|c|}{ Clad } & \multicolumn{2}{|c|}{ First Wall } \\
\hline & & $10^{+\infty}$ & & $2 \cdot 10^{+04}$ & $1.1 \cdot 10^{+c}$ \\
\hline & & & & & $0^{-c}$ \\
\hline & & & & & \\
\hline & & $\cdot 10^{+03}$ & & $2.0 \cdot 10^{+0 \theta}$ & 2.6 \\
\hline \multirow{5}{*}{$\begin{array}{l}\text { Radioactivity } \\
\gamma \text {-Dose Rate } \\
\text { Decay Power } \\
B H P\end{array}$} & \multirow{5}{*}{$\begin{array}{r}{[C i]} \\
{[S v / h]} \\
{[M W]} \\
{\left[m^{3}\right]} \\
\end{array}$} & \multicolumn{2}{|c|}{ Fuel } & \multicolumn{2}{|c|}{ Breeder Blanket } \\
\hline & & Fo1 & 5.8 & $2.0 \cdot 10^{+04}$ & $1.3 \cdot 10^{+0}$ \\
\hline & & & & & $1^{-0}$ \\
\hline & & & & & )$^{-0}$ \\
\hline & & & 3.4 & $2.0 \cdot 10^{+09}$ & $2.1 \cdot 10^{+08}$ \\
\hline \multirow{6}{*}{$\begin{array}{l}\text { Radioactivity } \\
\gamma \text {-Dose Rate } \\
\text { Decay Power } \\
B H P \\
\end{array}$} & \multirow{5}{*}{$\begin{array}{r}{[\mathrm{Ci}]} \\
{[S v / h]} \\
{[M W]} \\
{\left[\mathrm{m}^{3}\right]} \\
\end{array}$} & \multicolumn{2}{|c|}{ Duct } & \multicolumn{2}{|c|}{ Back Wall/Manifold } \\
\hline & & $2.2 \cdot 10^{+00}$ & $0^{+02}$ & $10^{+04}$ & $10^{+c}$ \\
\hline & & 39 & & $+\infty$ & $0^{-0}$ \\
\hline & & $\cdot 10^{-10}$ & 6.2 & $4 \cdot 10^{-05}$ & $0^{-0}$ \\
\hline & & $\cdot 10^{+04}$ & $7.6 \cdot 10^{+07}$ & $\cdot 10^{+09}$ & $10^{+c}$ \\
\hline & \multirow{5}{*}{$\begin{array}{r}{[C i]} \\
{[S v / h]} \\
{[M W]} \\
{\left[\mathrm{m}^{3}\right]} \\
\end{array}$} & \multicolumn{2}{|c|}{ Steel Shield } & \multicolumn{2}{|c|}{ Steel Shield } \\
\hline \multirow{4}{*}{$\begin{array}{l}\text { Radioactivity } \\
\gamma \text {-Dose Rate } \\
\text { Decay Power } \\
B H P \\
\end{array}$} & & $4.0 \cdot 10^{+01}$ & $1.4 \cdot 10^{+03}$ & $9.2 \cdot 10^{+03}$ & $1.5 \cdot 10^{+0}$ \\
\hline & & & & 1 & 1 . \\
\hline & & $10^{-09}$ & 1.5 & $9.1 \cdot 10^{-06}$ & $j^{-0}$ \\
\hline & & 0 & $1.8 \cdot 1$ & $\cdot 10^{+09}$ & $8.9 \cdot 10^{+0}$ \\
\hline \multirow{5}{*}{$\begin{array}{l}\text { Radioactivity } \\
\gamma \text {-Dose Rate } \\
\text { Decay Power } \\
B H P\end{array}$} & \multirow{5}{*}{$\begin{array}{r}{[C i]} \\
{[S v / h]} \\
{[M W]} \\
{\left[\mathrm{m}^{3}\right]} \\
\end{array}$} & \multicolumn{2}{|c|}{ Reactor } & \multicolumn{2}{|c|}{ Reactor } \\
\hline & & 1.0 & $8.5 \cdot 10^{+03}$ & $6.5 \cdot 10^{+04}$ & $1.4 \cdot 10^{+0}$ \\
\hline & & A & 8.1 & & \\
\hline & & $10^{-08}$ & 1.0 & $5.7 \cdot 10^{-08}$ & $1.1 \cdot 10^{-0}$ \\
\hline & & $3.6 \cdot 10$ & $7.2 \cdot 1$ & $7.0 \cdot 10$ & $2.4 \cdot 10^{+}$ \\
\hline
\end{tabular}

Table B.10: Absolute Radioactive Waste Parameters for Life Time Radioactive Waste at 1000 years after Discharge 


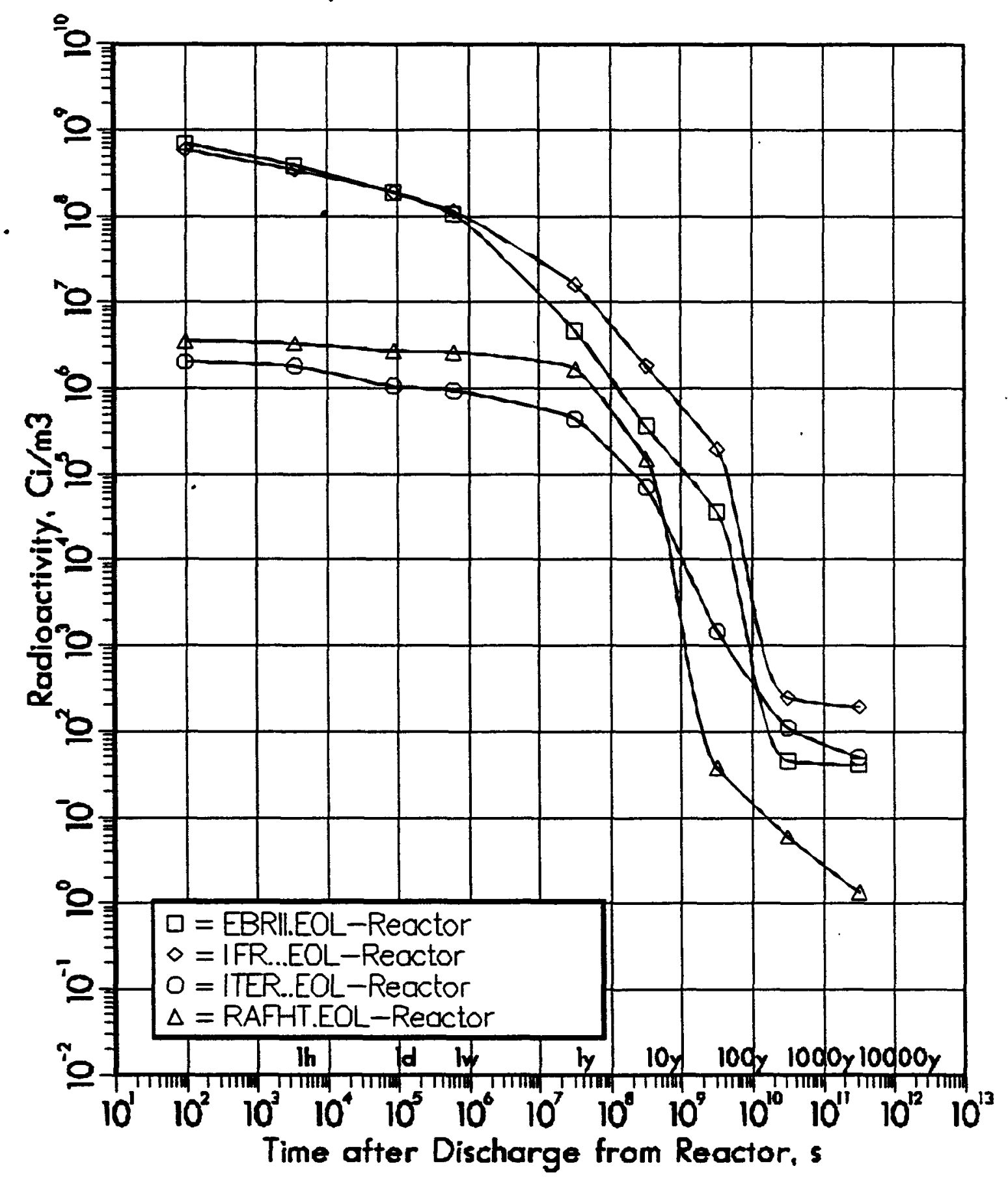

Figure B.1: Reactor Specific Radioactivity for EBR-II, IFR, ITER and $R A F H T$ 


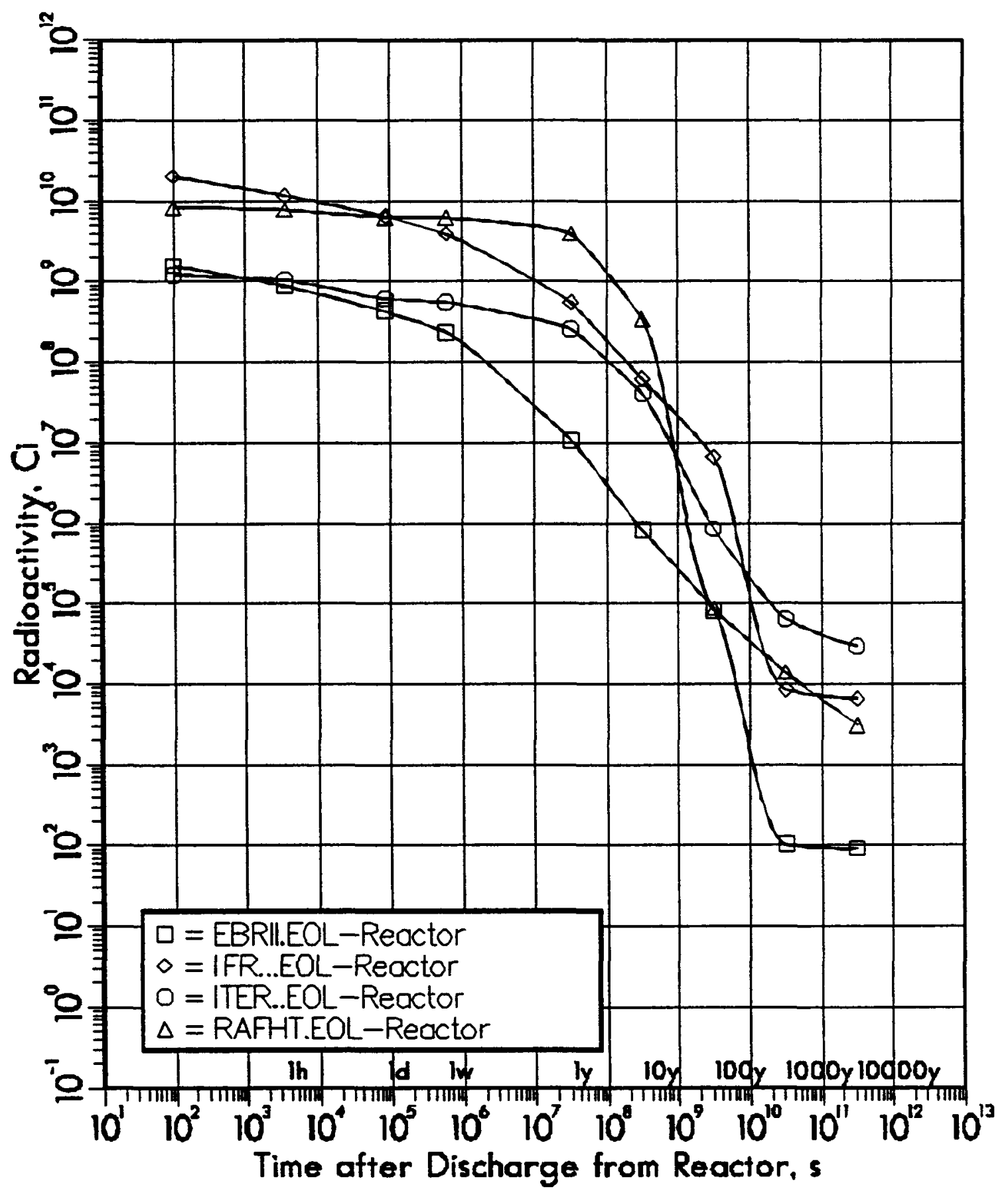

Figure B.2: Reactor Life Time Absolute Radioactivity for $E B R-I I, I F R$, ITER and RAFHT 


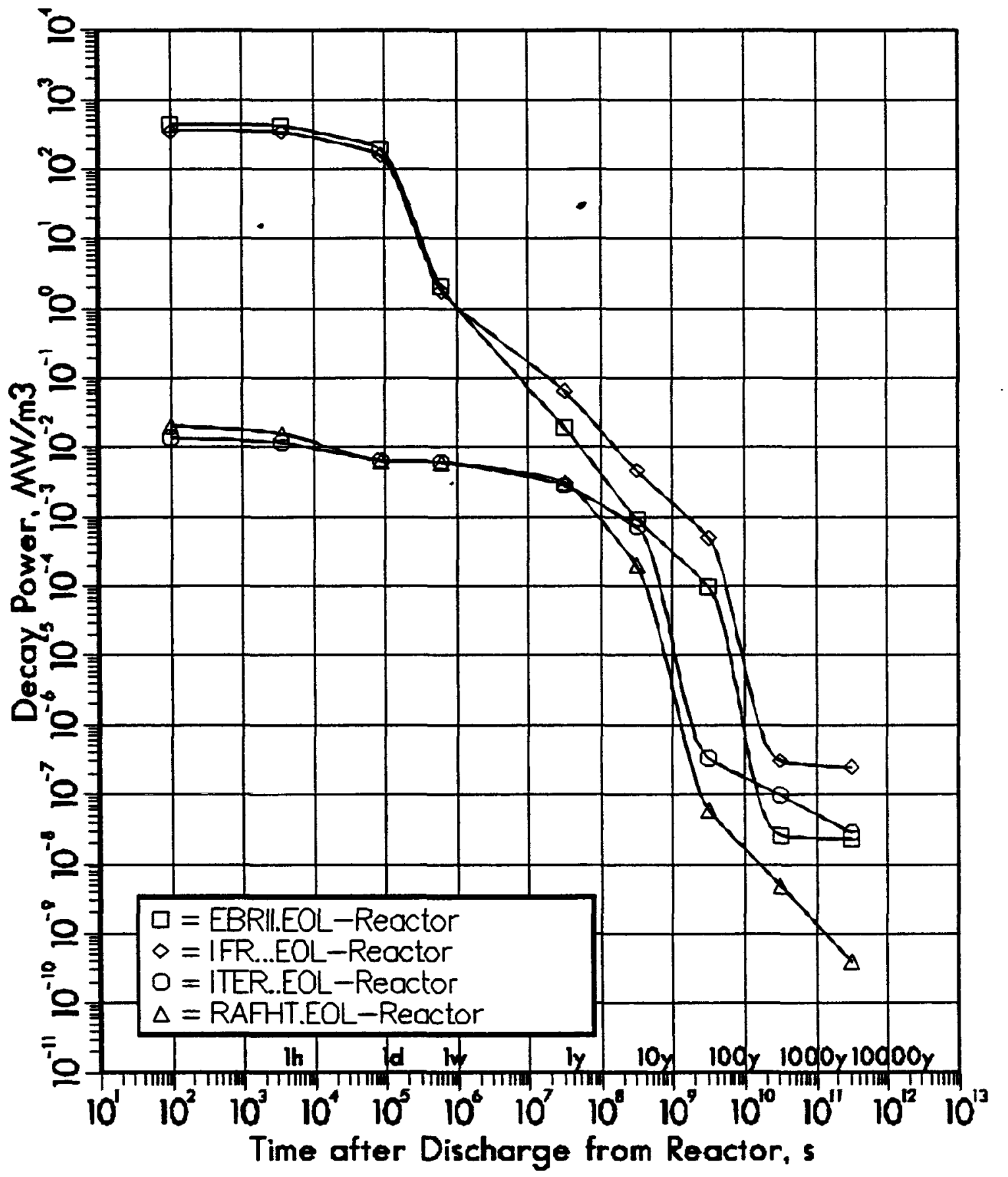

Figure B.3: Reactor Specific Decay Power for EBR-II, IFR, ITER and $R A F H T$ 


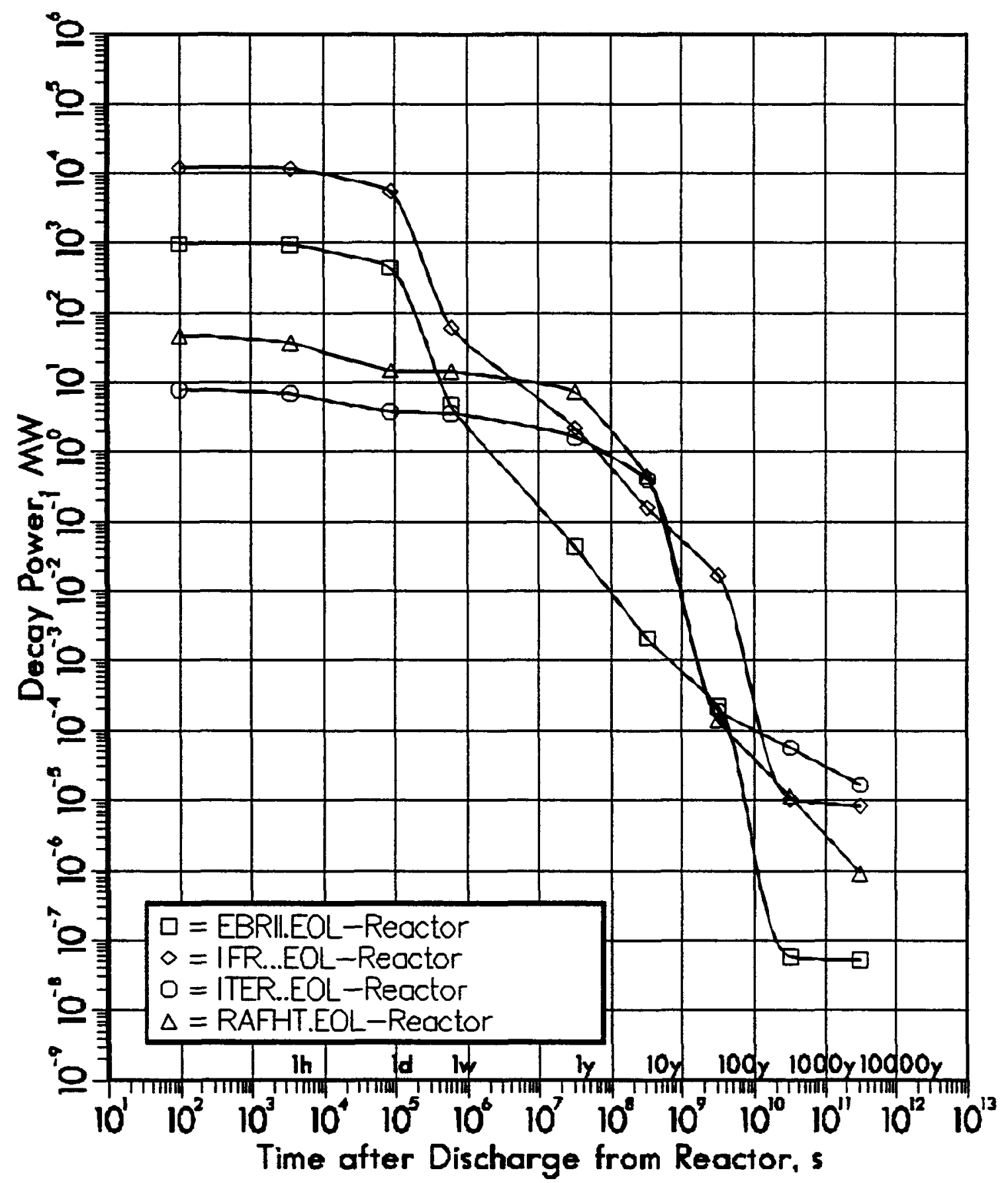

Figure B.4: Reactor Life Time Absolute Decay Power for EBR-II, IFR, ITER and RAFHT 